\title{
Ru(II)-Catalyzed Amination of Aryl Fluorides via $\eta^{6}$-Coordination
}

\author{
Qi-Kai Kang, ${ }^{\dagger, \dot{\ddagger}}$ Yunzhi Lin, ${ }^{\dagger, \dot{\dagger}}$ Yuntong $\mathrm{Li}^{\dagger, \dot{\dagger}}$ and Hang Shi ${ }^{*}, \dot{\dagger},+$ \\ 'Institute of Natural Sciences, Westlake Institute for Advanced Study, 18 Shilongshan Road, \\ Hangzhou 310024, Zhejiang Province, China. \\ ${ }^{\ddagger}$ Key Laboratory of Precise Synthesis of Functional Molecules of Zhejiang Province, School \\ of Science, Westlake University, 18 Shilongshan Road, Hangzhou 310024, Zhejiang Province, \\ China.
}

\section{TABLE OF CONTENTS}

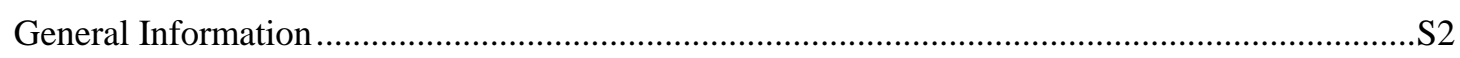

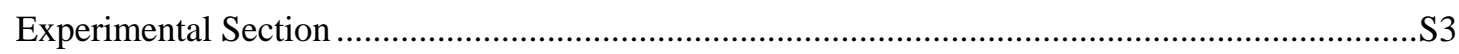

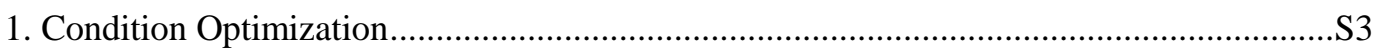

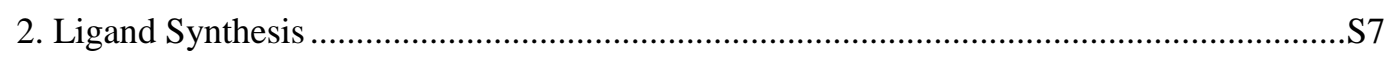

3. Ru(II)-Catalyzed Amination of Aryl Fluorides ................................................................. 9

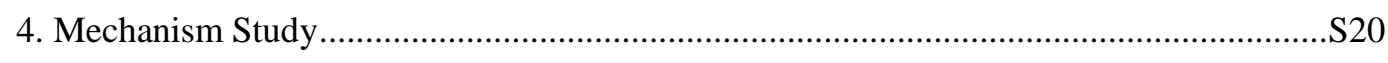

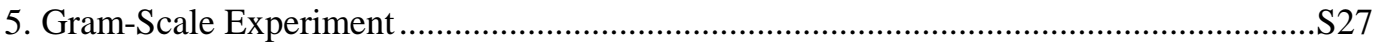

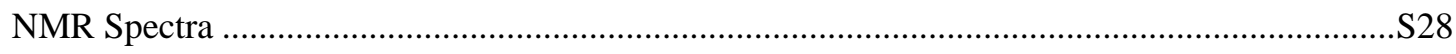

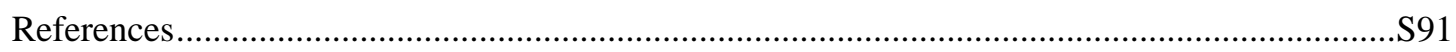




\section{General Information}

\section{Solvents}

Dry dichloromethane (DCM), tetrahydrofuran (THF), 1,4-dioxane, 1,2-dimethoxyethane (DME) and N,N-dimethylformamide (DMF) were purchased from Energy Chemical Company. Chloroform- $d_{1}$ (containing 0.03\% TMS) and acetone- $d_{6}$ (containing $0.03 \%$ TMS) were purchased from $J \& K$ Chemical Company.

\section{Chromatography}

Analytical thin layer chromatography was performed on $0.25 \mathrm{~mm}$ silica gel $60-\mathrm{F} 254$. Visualization was carried out with UV light.

\section{Spectroscopy and Instruments}

${ }^{1} \mathrm{H}$ NMR was recorded on Bruker AVANCE NEO instrument (500 and 600MHz). Chemical shifts were quoted in parts per million (ppm) referenced with tetramethylsilane $(\delta=0.00 \mathrm{ppm})$ in chloroform- $d_{1}$ or acetone- $d_{6} \cdot{ }^{13} \mathrm{C}$ NMR spectra were recorded on Bruker AVANCE NEO instrument (125 and $150 \mathrm{MHz}$ ), and were fully decoupled by broad band proton decoupling. Chemical shifts were reported in ppm referenced to the center peak of a triplet at $77.00 \mathrm{ppm}$ of chloroform- $d_{l} \cdot{ }^{19} \mathrm{~F}$ NMR spectra were recorded on Bruker AVANCE NEO instrument $(470 \mathrm{MHz}) .{ }^{31} \mathrm{P}$ NMR spectra were recorded on Bruker AVANCE NEO instrument (202 MHz). The following abbreviations (or combinations thereof) were used to explain multiplicities: $\mathrm{s}=$ singlet, $\mathrm{d}=$ doublet, $\mathrm{t}=$ triplet, $\mathrm{m}=$ multiplet, $\mathrm{br}=$ broad. Coupling constants, $J$, were reported in Hertz unit $(\mathrm{Hz})$. High-resolution mass spectra (HRMS) were recorded on a Waters Mass spectrometer using ESI-TOF (electrospray ionization-time of flight).

\section{Starting materials}

All substrates were used as received from commercial suppliers, unless otherwise stated. $\left[\mathrm{Ru}(\text { cymene }) \mathrm{Cl}_{2}\right]_{2}$ and silver salts were purchased from Energy Chemical Company. Molecular sieves were purchased from Sigma-Aldrich. Ligands L1-L4 and L15 were purchased from Energy Chemical Company, L5-L14 and L16-L18 are known compounds and synthesized according to reported literatures. ${ }^{1}$ 


\section{Experimental Section}

\section{Condition Optimization}

\section{Table S1. Effect of Ag salts ${ }^{\mathrm{a}}$}

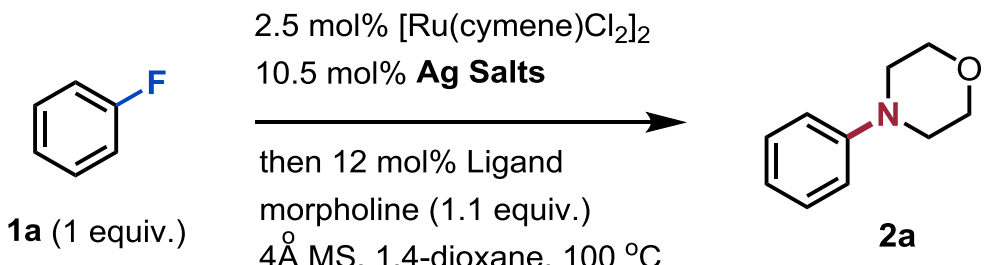

\begin{tabular}{|c|c|c|c|}
\hline Entry & Ligand & Ag salt & Yield $(\%)$ \\
\hline 1 & $\mathrm{Me}_{(\mathbf{L})}$ & $\mathrm{AgPF}_{6}$ & 42 \\
\hline 2 & $\mathrm{Me}_{\text {(L6) }}$ & AgOTf & 38 \\
\hline 3 & $\mathrm{le}_{(\mathrm{L})}$ & $\mathrm{AgSbF}_{6}$ & 12 \\
\hline 4 & $\mathrm{Me}$ (L6) & $\mathrm{AgBF}_{4}$ & 17 \\
\hline 5 & ${ }^{\mathrm{OMe}}{ }_{(\mathbf{L} 6)}$ & $\mathrm{AgOAc}$ & N.R \\
\hline
\end{tabular}

${ }^{\mathrm{a} C}$ Conditions: 1a $(0.40 \mathrm{mmol})$, morpholine $(0.44 \mathrm{mmol}),\left[\mathrm{Ru}(\text { cymene }) \mathrm{Cl}_{2}\right]_{2}(0.010 \mathrm{mmol}), \mathrm{Ag}$ salts $(0.042 \mathrm{mmol})$, Ligand $(0.048 \mathrm{mmol}), 4 \AA \mathrm{MS}(20 \mathrm{mg}), 1$,4-dioxane $(0.10 \mathrm{~mL}), 100{ }^{\circ} \mathrm{C}$. Yield was determined by ${ }^{1} \mathrm{H}$ NMR using 1,1,2,2-tetrachloroethane as the internal standard.

\section{Table S2. Effect of Temperature}

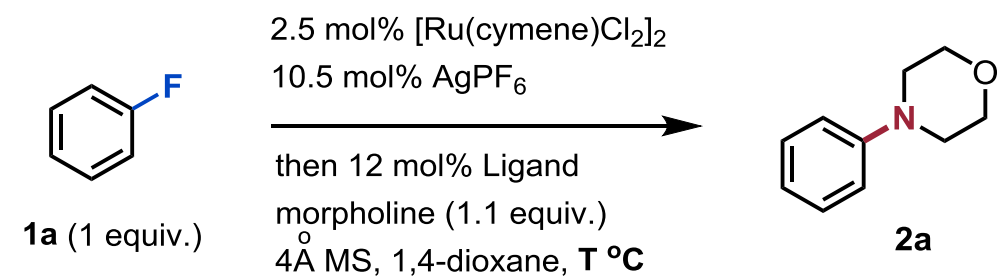

\begin{tabular}{|c|c|c|c|}
\hline Entry & Ligand & $\mathrm{T}\left({ }^{\circ} \mathrm{C}\right)$ & Yield $(\%)$ \\
\hline 1 & & 100 & 42 \\
\hline 2 & & 120 & 46 \\
\hline
\end{tabular}


${ }^{\mathrm{a} C}$ Conditions: $1 \mathrm{a}(0.40 \mathrm{mmol})$, morpholine $(0.44 \mathrm{mmol}),\left[\mathrm{Ru}(\mathrm{cymene}) \mathrm{Cl}_{2}\right]_{2}(0.010 \mathrm{mmol}), \mathrm{AgPF}_{6}(0.042 \mathrm{mmol})$, Ligand $(0.048 \mathrm{mmol}), 4 \AA \mathrm{MS}(20 \mathrm{mg})$, 1,4-dioxane $(0.10 \mathrm{~mL})$. Yield was determined by ${ }^{1} \mathrm{H}$ NMR using 1,1,2,2-tetrachloroethane as the internal standard.

Table S3. Effect of Ligand/Metal Ratio ${ }^{\text {a }}$

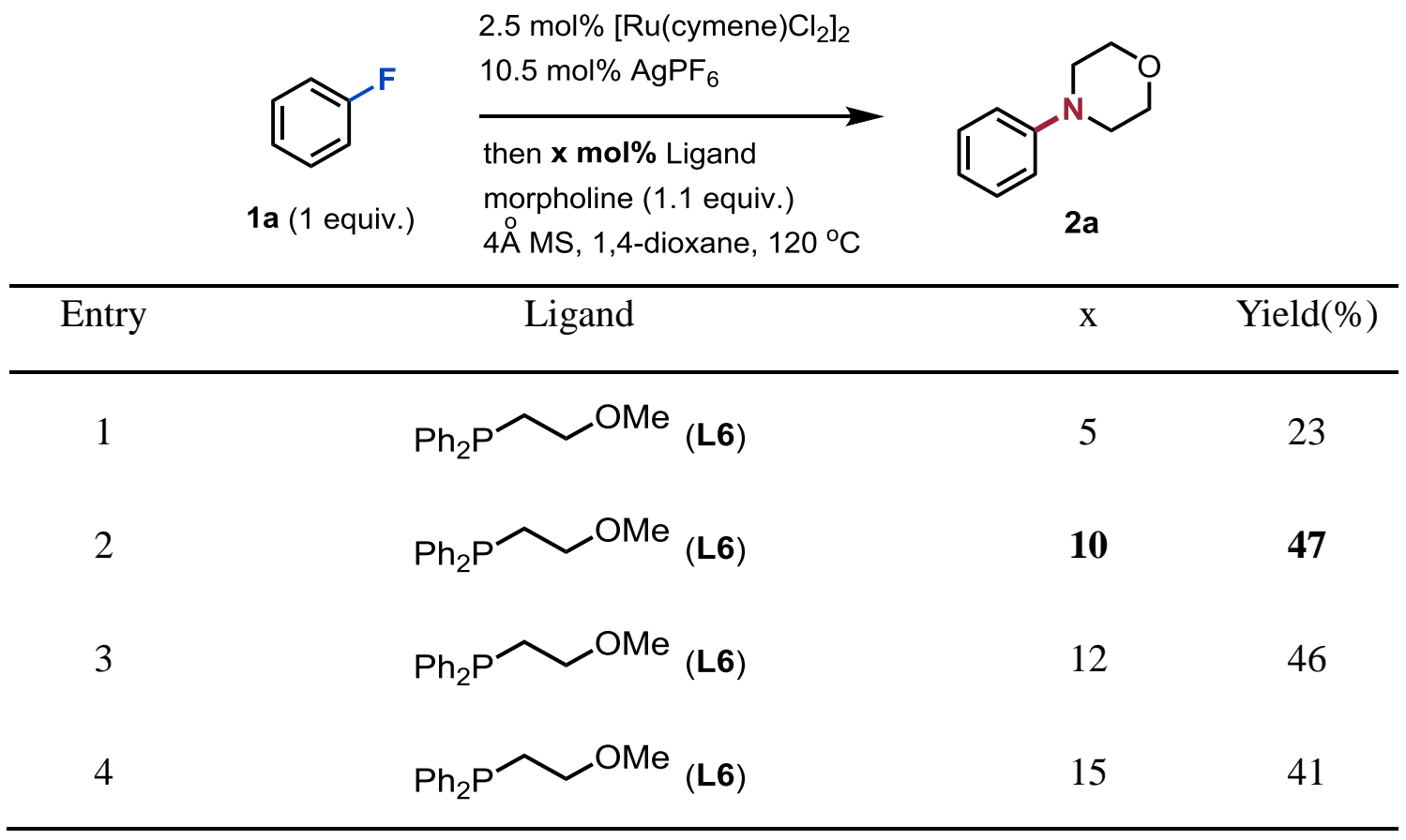

${ }^{\mathrm{a}}$ Conditions: 1a $(0.40 \mathrm{mmol})$, morpholine $(0.44 \mathrm{mmol}),\left[\mathrm{Ru}(\text { cymene }) \mathrm{Cl}_{2}\right]_{2}(0.010 \mathrm{mmol}), \mathrm{AgPF}_{6}(0.042 \mathrm{mmol})$, Ligand (x mmol), $4 \AA$ MS $(20 \mathrm{mg}), 1,4$-dioxane $(0.10 \mathrm{~mL}), 120{ }^{\circ} \mathrm{C}$. Yield was determined by ${ }^{1} \mathrm{H}$ NMR using 1,1,2,2-tetrachloroethane as the internal standard.

Table S4. Effect of Amine Equivalent ${ }^{\mathrm{a}}$

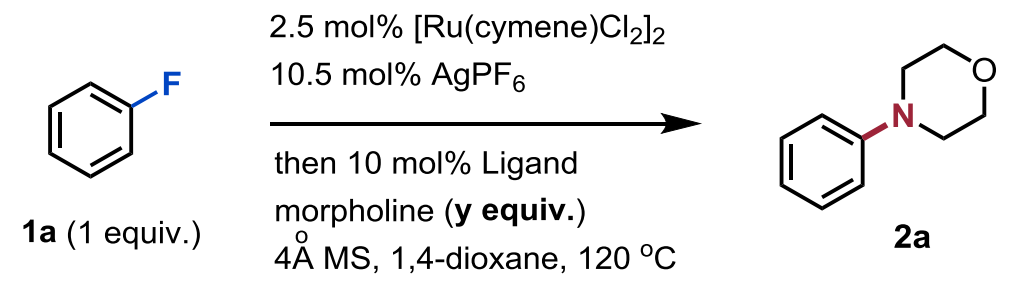

\begin{tabular}{cccc}
\hline Entry & Ligand & y & Yield(\%) \\
\hline 1 & $\mathrm{Ph}_{2} \mathrm{P} \sim \sim^{\sim \text { OMe }}{ }_{(\text {L6) }}$ & 1.1 & 47
\end{tabular}


${ }^{\mathrm{a} C}$ Conditions: 1a $(0.40 \mathrm{mmol})$, morpholine (y equiv.), $\left[\mathrm{Ru}(\text { cymene }) \mathrm{Cl}_{2}\right]_{2}(0.010 \mathrm{mmol}), \mathrm{AgPF} 6(0.042 \mathrm{mmol})$, Ligand $(0.040 \mathrm{mmol}), 4 \AA \mathrm{MS}(20 \mathrm{mg}), 1,4$-dioxane $(0.10 \mathrm{~mL}), 120{ }^{\circ} \mathrm{C}$. Yield was determined by $1 \mathrm{H}$ NMR using 1,1,2,2-tetrachloroethane as the internal standard.

Table S5. Effect of Solvent and PhF Concentration ${ }^{\text {a }}$

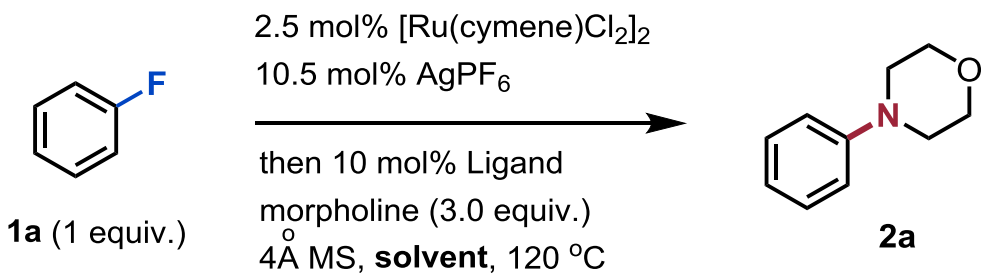

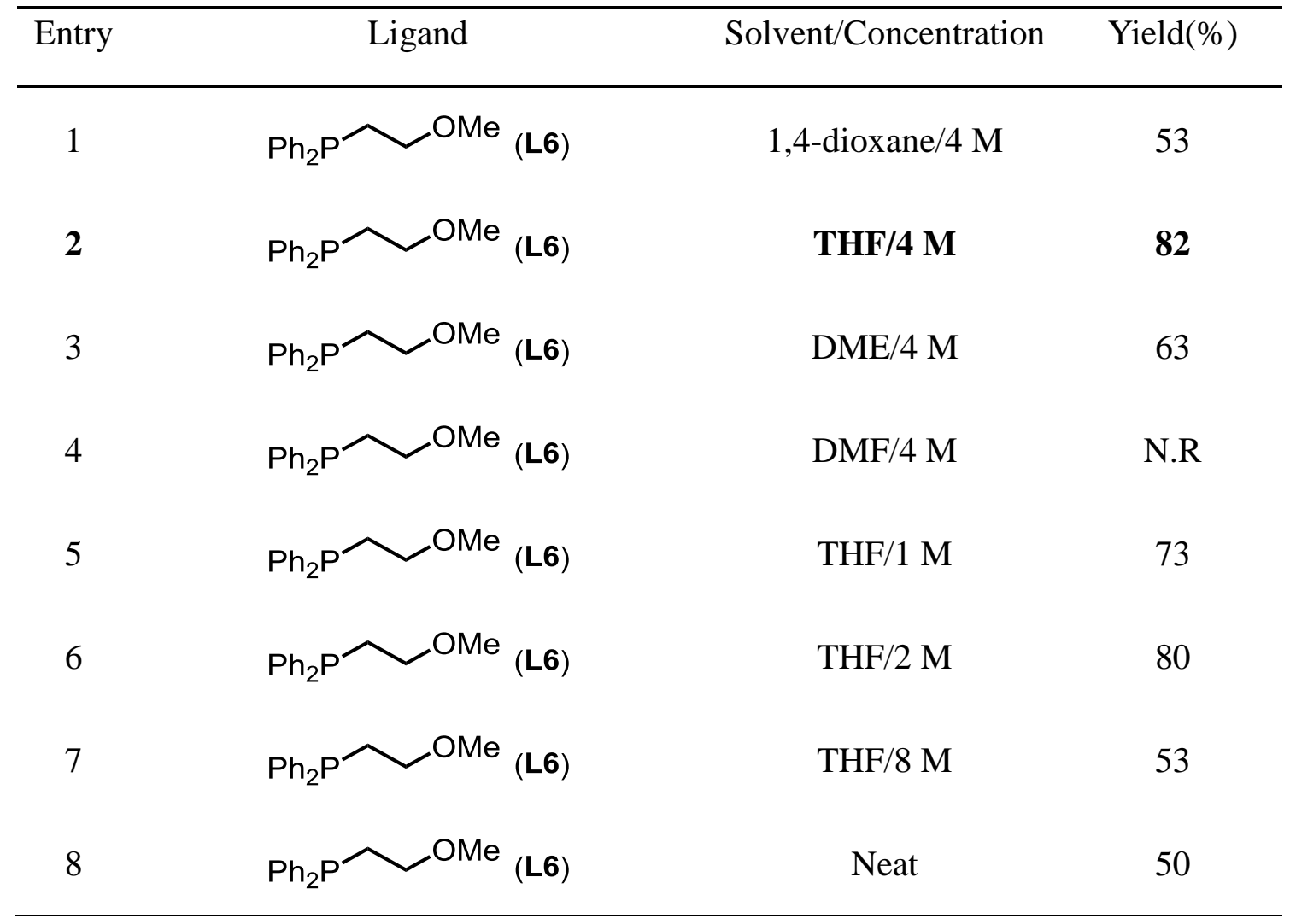

${ }^{\mathrm{a} C}$ Conditions: $1 \mathrm{a}(0.40 \mathrm{mmol})$, morpholine $(1.20 \mathrm{mmol}),\left[\mathrm{Ru}(\mathrm{cymene}) \mathrm{Cl}_{2}\right]_{2}(0.010 \mathrm{mmol}), \mathrm{AgPF}_{6}(0.042 \mathrm{mmol})$, Ligand $(0.040 \mathrm{mmol}), 4 \AA \mathrm{MS}(20 \mathrm{mg})$, solvent, $120{ }^{\circ} \mathrm{C}$. Yield was determined by ${ }^{1} \mathrm{H}$ NMR using 1,1,2,2-tetrachloroethane as the internal standard.

Table S6. Effect of Additives ${ }^{\text {a }}$ 


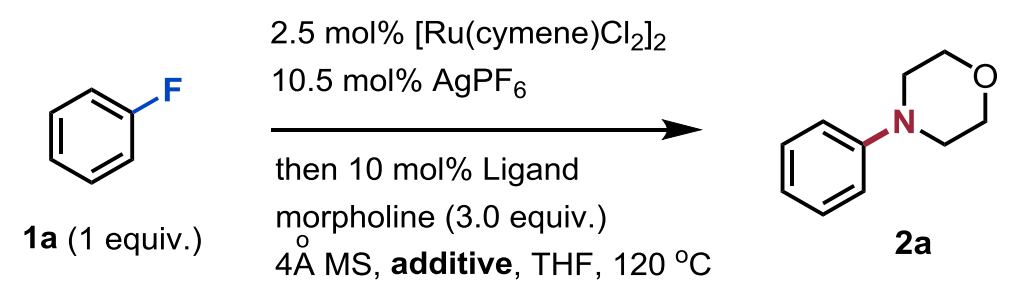

\begin{tabular}{|c|c|c|c|}
\hline Entry & Ligand & Additive & Yield $(\%)$ \\
\hline 1 & OMe (L6) & -- & 82 \\
\hline 2 & (L6) & $\mathrm{Et}_{3} \mathrm{~N}$ & 70 \\
\hline 3 & $\mathrm{Me}_{(\mathbf{L})}$ & $\mathrm{CsF}$ & 11 \\
\hline 4 & ${ }_{(\mathrm{OMe})}$ & $\mathrm{Na}_{2} \mathrm{CO}_{3}$ & 23 \\
\hline 5 & ${ }^{\mathrm{OMe}}{ }_{(\mathrm{L} 6)}$ & $\mathrm{Et}_{3} \mathrm{SiH}$ & N.R \\
\hline
\end{tabular}

${ }^{\mathrm{a} C}$ Conditions: $1 \mathrm{a}(0.40 \mathrm{mmol})$, morpholine $(1.20 \mathrm{mmol}),\left[\mathrm{Ru}(\mathrm{cymene}) \mathrm{Cl}_{2}\right]_{2}(0.010 \mathrm{mmol}), \mathrm{AgPF}_{6}(0.042 \mathrm{mmol})$, Ligand $(0.040 \mathrm{mmol}), 4 \AA \mathrm{MS}(20 \mathrm{mg})$, additive $(0.40 \mathrm{mmol})$, THF $(0.10 \mathrm{~mL}), 120{ }^{\circ} \mathrm{C}$. Yield was determined by ${ }^{1} \mathrm{H}$ NMR using 1,1,2,2-tetrachloroethane as the internal standard.

\section{Table S7. Effect of Ligands ${ }^{\mathrm{a}}$}
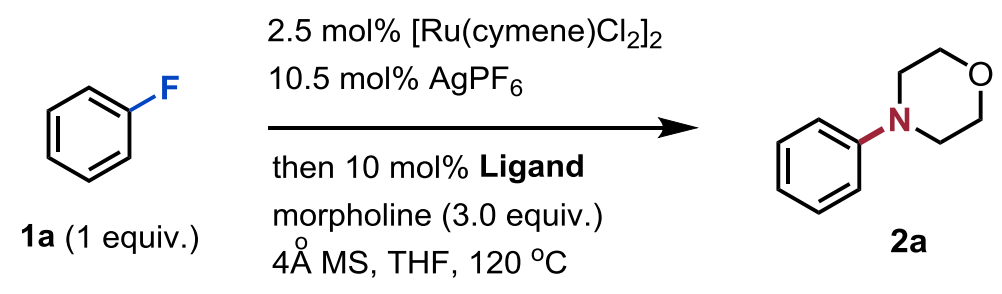

$$
\text { OMe }
$$<smiles>Fc1ccc(I)cc1</smiles><smiles>Cc1cc(F)cc(F)c1</smiles><smiles>COc1ccc(I)cc1</smiles><smiles>[13CH3][18OH]</smiles><smiles>[13CH3][13CH3]</smiles>

L20 (54\%)

L21 (12\%)

L22 (95\%)

$\mathbf{L} 23(33 \%)$

${ }^{\mathrm{a}}$ Conditions: $1 \mathrm{a}(0.40 \mathrm{mmol})$, morpholine $(1.20 \mathrm{mmol}),\left[\mathrm{Ru}(\mathrm{cymene}) \mathrm{Cl}_{2}\right]_{2}(0.010 \mathrm{mmol}), \mathrm{AgPF}_{6}(0.042 \mathrm{mmol})$, Ligand $(0.040 \mathrm{mmol}), 4 \AA \mathrm{MS}(20 \mathrm{mg})$, THF $(0.10 \mathrm{~mL}), 120{ }^{\circ} \mathrm{C}$. Yield was determined by ${ }^{1} \mathrm{H}$ NMR using 1,1,2,2-tetrachloroethane as the internal standard. 


\section{Ligand Synthesis}

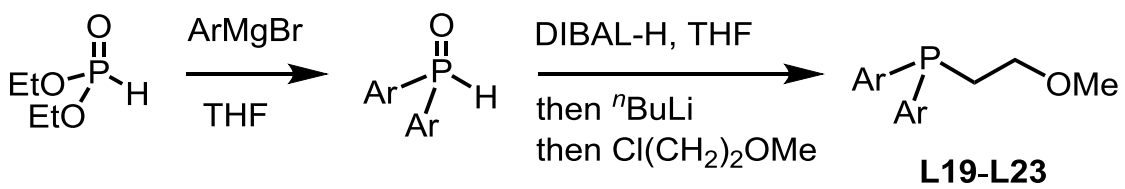

Diethyl phosphate $(10 \mathrm{mmol})$ was dissolved in $20 \mathrm{~mL}$ THF under nitrogen atmosphere, and then Grignard reagent $(30 \mathrm{mmol})$ was added slowly to the solution at $0^{\circ} \mathrm{C}$. The mixture was allowed to warm to room temperature gradually and stirred at room temperature until the disappearance of starting material. The reaction was quenched by saturated $\mathrm{NH}_{4} \mathrm{Cl}$ solution and extracted by DCM (30 $\mathrm{mL}$ x 3$)$. The organic phase was separated, dried by $\mathrm{Na}_{2} \mathrm{SO}_{4}$ and concentrated. The crude product was further purified by chromatography on silica gel (ethyl acetate/ petroleum ether).

The obtained phosphine oxide $(5 \mathrm{mmol})$ was dissolved in $30 \mathrm{~mL}$ THF under nitrogen atmosphere and cooled to $0^{\circ} \mathrm{C}$, then $1.0 \mathrm{M}$ DIBAL-H in hexane $(15 \mathrm{mmol})$ was added and the mixture was stirred at $0^{\circ} \mathrm{C}$ for 30 minutes. Without isolating the obtained diarylphosphane, $1.6 \mathrm{M} n$-BuLi in hexane $(15 \mathrm{mmol})$ was added directly, and then 2-chloroethyl methyl ether was added after 30 minutes. The reaction was stirred for another 1 hour and quenched by $15 \mathrm{~mL} \mathrm{H}_{2} \mathrm{O}$. The mixture was extracted by DCM (30 $\mathrm{mL} \times 3$ ), and then the organic phase was separated, dried by $\mathrm{Na}_{2} \mathrm{SO}_{4}$ and concentrated. The crude product was further purified by chromatography on silica gel (ethyl acetate/petroleum ether $=30 / 1$ ), affording the expected product.<smiles>COCCP(c1ccccc1C)c1ccccc1C</smiles>

L19

Colourless oil, 60\% yield, ${ }^{1} \mathrm{H}$ NMR $\left(500 \mathrm{MHz}, \mathrm{CDCl}_{3}\right): \delta 7.24-7.20(\mathrm{~m}, 4 \mathrm{H})$, 7.17-7.14 (m, 4H), 3.51-3.47 (m, 2H), 3.31 (s, 3H), 2.41 (s, 6H), 2.32 (t, $J=7.0 \mathrm{~Hz}, 2$ $\mathrm{H}) ;{ }^{13} \mathrm{C}$ NMR $\left(125 \mathrm{MHz}, \mathrm{CDCl}_{3}\right): \delta 142.3(\mathrm{~d}, J=26.9 \mathrm{~Hz}), 136.42-136.32(\mathrm{~m}), 131.1$, $130.0(\mathrm{~d}, J=4.5 \mathrm{~Hz}), 128.5,126.1,69.8(\mathrm{~d}, J=24.8 \mathrm{~Hz}), 58.5,27.65-27.51(\mathrm{~m}), 21.2$ $(\mathrm{d}, J=22.1 \mathrm{~Hz}) ;{ }^{31} \mathrm{P}$ NMR $\left(202 \mathrm{MHz}, \mathrm{CDCl}_{3}\right):-44.1$; HRMS-ESI $(\mathrm{m} / \mathrm{z}):[\mathrm{M}+\mathrm{H}]^{+}$ calcd. for $\left[\mathrm{C}_{17} \mathrm{H}_{22} \mathrm{OP}\right]^{+}, 273.1403$; found, 273.1418 .

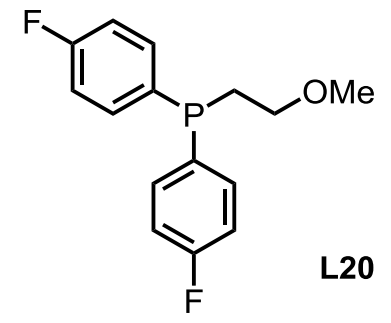

Colourless oil, 59\% yield, ${ }^{1} \mathrm{H}$ NMR $\left(500 \mathrm{MHz}, \mathrm{CDCl}_{3}\right): \delta 7.41-7.37(\mathrm{~m}, 4 \mathrm{H})$, 7.06-7.02 (m, 4H), 3.50-3.45 (m, 2H), $3.30(\mathrm{~s}, 3 \mathrm{H}), 2.34(\mathrm{t}, J=7.5 \mathrm{~Hz}, 2 \mathrm{H}) ;{ }^{13} \mathrm{C}$ 
NMR (125 MHz, $\left.\mathrm{CDCl}_{3}\right): \delta 163.3(\mathrm{~d}, J=248.1 \mathrm{~Hz}), 134.6-134.4(\mathrm{~m}), 133.6-133.5$ (m), 115.85-115.62 (m), $69.6(\mathrm{~d}, J=23.1 \mathrm{~Hz}), 58.6,29.3(\mathrm{~d}, J=12.5 \mathrm{~Hz}) ;{ }^{31} \mathrm{P}$ NMR $\left(202 \mathrm{MHz}, \mathrm{CDCl}_{3}\right):-24.2 ;{ }^{19} \mathrm{~F}$ NMR $\left(470 \mathrm{MHz}, \mathrm{CDCl}_{3}\right):-112.34--112.37$ (m); HRMS-ESI (m/z): $[\mathrm{M}+\mathrm{H}]^{+}$calcd. for $\left[\mathrm{C}_{15} \mathrm{H}_{16} \mathrm{OF}_{2} \mathrm{P}\right]^{+}, 281.0901$; found, 281.0901.<smiles>COCCP(c1cc(F)cc(F)c1)c1cc(F)cc(F)c1</smiles>

Colourless oil, $40 \%$ yield, ${ }^{1} \mathrm{H}$ NMR $\left(500 \mathrm{MHz}, \mathrm{CDCl}_{3}\right): \delta 6.94-6.91(\mathrm{~m}, 4 \mathrm{H})$, 6.82-6.78 (m, 2H), 3.54-3.49 (m, 2H), $3.32(\mathrm{~s}, 3 \mathrm{H}), 2.33(\mathrm{t}, J=7.5 \mathrm{~Hz}, 2 \mathrm{H}) ;{ }^{13} \mathrm{C}$ NMR (125 MHz, $\left.\mathrm{CDCl}_{3}\right): \delta 164.0-161.9(\mathrm{~m}), 142.0(\mathrm{~d}, J=18.5 \mathrm{~Hz}), 115.3-115.0(\mathrm{~m})$, $104.7(\mathrm{t}, J=25.9 \mathrm{~Hz}), 69.1(\mathrm{~d}, J=20.8 \mathrm{~Hz}), 58.7,28.7(\mathrm{~d}, J=14.6 \mathrm{~Hz}) ;{ }^{31} \mathrm{P} \mathrm{NMR}$ $\left(202 \mathrm{MHz}, \mathrm{CDCl}_{3}\right):-16.25--16.42(\mathrm{~m}) ;{ }^{19} \mathrm{~F} \mathrm{NMR}\left(470 \mathrm{MHz}, \mathrm{CDCl}_{3}\right)$ : $-108.58-$ -108.61 (m); HRMS-ESI (m/z): $[\mathrm{M}+\mathrm{H}]^{+}$calcd. for $\left[\mathrm{C}_{15} \mathrm{H}_{14} \mathrm{OF}_{4} \mathrm{P}\right]^{+}, 317.0713$; found, 317.0713 .

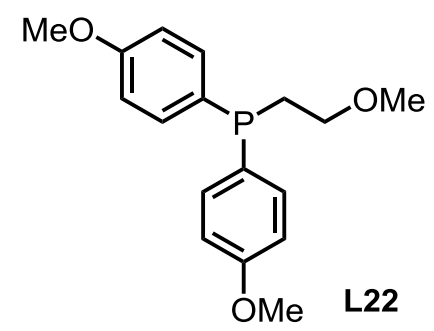

Colourless oil, $70 \%$ yield, ${ }^{1} \mathrm{H}$ NMR $\left(500 \mathrm{MHz}, \mathrm{CDCl}_{3}\right): \delta 7.37-7.34(\mathrm{~m}, 4 \mathrm{H})$, 6.89-6.87 (m, 4H), $3.80(\mathrm{~s}, 6 \mathrm{H}), 3.49-3.44(\mathrm{~m}, 2 \mathrm{H}), 3.30(\mathrm{~s}, 3 \mathrm{H}), 2.31(\mathrm{t}, J=7.5 \mathrm{~Hz}$, $2 \mathrm{H}) ;{ }^{13} \mathrm{C}$ NMR $\left(125 \mathrm{MHz}, \mathrm{CDCl}_{3}\right): \delta 160.1,134.0(\mathrm{~d}, J=20.3 \mathrm{~Hz}), 129.0,114.1(\mathrm{~d}, J$ $=7.8 \mathrm{~Hz}), 69.8(\mathrm{~d}, J=23 \mathrm{~Hz}), 58.5,55.1,29.2(\mathrm{~d}, J=11.4 \mathrm{~Hz}) ;{ }^{31} \mathrm{P}$ NMR $(202 \mathrm{MHz}$, $\left.\mathrm{CDCl}_{3}\right)$ : -26.0; HRMS-ESI (m/z): $[\mathrm{M}+\mathrm{H}]^{+}$calcd. for $\left[\mathrm{C}_{17} \mathrm{H}_{22} \mathrm{O}_{3} \mathrm{P}\right]^{+}, 305.1301$; found, 305.1306 .<smiles>COCCP(c1ccc2c(c1)OCO2)c1ccc2c(c1)OCO2</smiles>

Colourless oil, 55\% yield, ${ }^{1} \mathrm{H}$ NMR $\left(500 \mathrm{MHz}, \mathrm{CDCl}_{3}\right): \delta 6.90-6.86(\mathrm{~m}, 2 \mathrm{H})$, 6.77-6.76 (m, 2H), 6.71-6.69 (m, 2H), $5.83(\mathrm{~s}, 4 \mathrm{H}), 3.40-3.35(\mathrm{~m}, 2 \mathrm{H}), 3.21(\mathrm{~s}, 3 \mathrm{H})$, $2.18(\mathrm{t}, J=7.5 \mathrm{~Hz}, 2 \mathrm{H}) ;{ }^{13} \mathrm{C} \mathrm{NMR}\left(125 \mathrm{MHz}, \mathrm{CDCl}_{3}\right): \delta 148.3,147.8(\mathrm{~d}, J=7.1 \mathrm{~Hz})$, $131.1(\mathrm{~d}, J=13.1 \mathrm{~Hz}), 127.0(\mathrm{~d}, J=27.1 \mathrm{~Hz}), 111.6(\mathrm{~d}, J=16.6 \mathrm{~Hz}), 108.5(\mathrm{~d}, J=$ $10.1 \mathrm{~Hz}), 100.9,69.6(\mathrm{~d}, J=10.1 \mathrm{~Hz}), 58.4,29.2(\mathrm{~d}, J=12.8 \mathrm{~Hz}) ;{ }^{31} \mathrm{P}$ NMR $(202$ $\mathrm{MHz}, \mathrm{CDCl}_{3}$ ): -20.4 - -20.6 (m); HRMS-ESI (m/z): $[\mathrm{M}+\mathrm{H}]^{+}$calcd. for $\left[\mathrm{C}_{17} \mathrm{H}_{18} \mathrm{O}_{5} \mathrm{P}\right]^{+}$, 333.0886; found, 333.0886 . 


\section{Ru(II)-Catalyzed Amination of Aryl Fluorides}

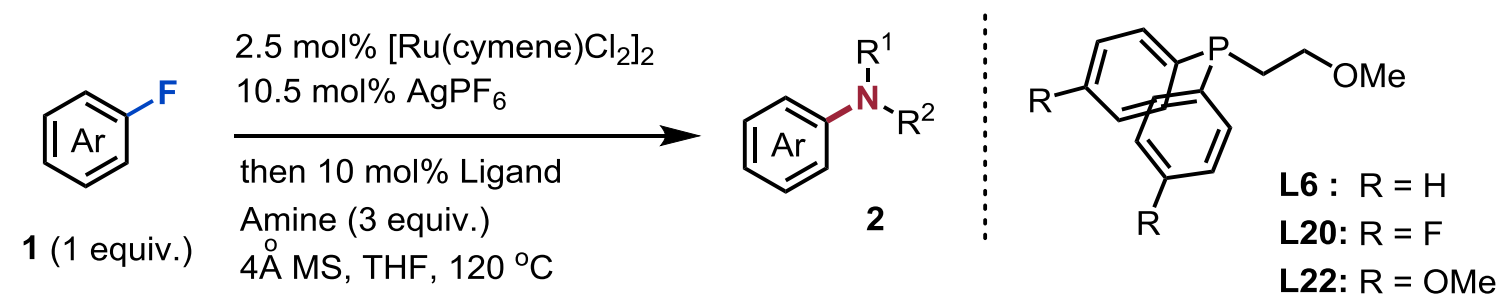

Catalyst Preparation: under $\mathrm{N}_{2}$ atmosphere, $\left[\mathrm{Ru}(\mathrm{cymene}) \mathrm{Cl}_{2}\right]_{2}(0.010 \mathrm{mmol})$ and $\mathrm{AgPF}_{6}(0.042 \mathrm{mmol})$ were stirred in acetone $(1.0 \mathrm{~mL})$ at room temperature for 15 minutes. The formed $\mathrm{AgCl}$ precipitate was filtered off and acetone solution was transferred to a $4 \mathrm{~mL}$ vial which contained ligand $(0.040 \mathrm{mmol})$. Then acetone was excluded under reduced pressure, affording the catalyst as yellow foam.

The prepared catalyst was mixed with $4 \AA$ MS $(20 \mathrm{mg})$, aryl fluoride $(0.4 \mathrm{mmol})$ and amine $(1.20 \mathrm{mmol})$ under $\mathrm{N}_{2}$ atmosphere, and then $0.1 \mathrm{~mL}$ THF was added. The reaction was heated to $120{ }^{\circ} \mathrm{C}$ and stirred for 20 to 48 hours. The mixture was purified by silica gel chromatography (ethyl acetate/ petroleum ether).

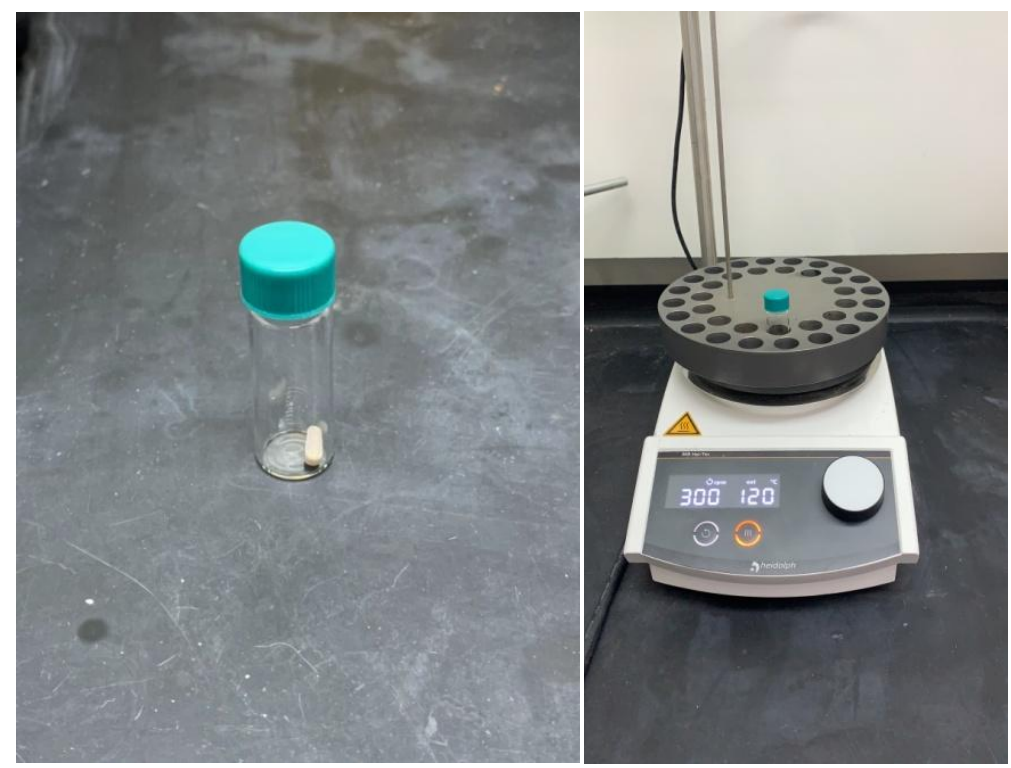

Figure S1. Reaction vessel<smiles>c1ccc(N2CCOCC2)cc1</smiles>

20 hours, 95\% yield (using L22 as ligand), known compound ${ }^{2 \mathrm{a}} ;{ }^{1} \mathrm{H}$ NMR (500 MHz, $\left.\mathrm{CDCl}_{3}\right): \delta 7.30-7.26(\mathrm{~m}, 2 \mathrm{H}), 6.93-6.87(\mathrm{~m}, 3 \mathrm{H}), 3.87(\mathrm{t}, J=5.0 \mathrm{~Hz}, 4 \mathrm{H}), 3.16(\mathrm{t}, J=$ $5.0 \mathrm{~Hz}, 4 \mathrm{H})$. 
<smiles>COc1ccccc1N1CCOCC1</smiles>

2b

20 hours, 62\% yield (using L20 as ligand), known compound ${ }^{2 \mathrm{a}} ;{ }^{1} \mathrm{H}$ NMR (500 MHz, $\left.\mathrm{CDCl}_{3}\right): \delta 7.04-7.00(\mathrm{~m}, 1 \mathrm{H}), 6.94-6.93(\mathrm{~m}, 2 \mathrm{H}), 6.89-6.87(\mathrm{~m}, 1 \mathrm{H}), 3.90(\mathrm{t}, J=5.0$ $\mathrm{Hz}, 4 \mathrm{H}), 3.87$ (s, 3H), 3.07 (t, $J=5.0 \mathrm{~Hz}, 4 \mathrm{H})$.<smiles>Nc1ccccc1N1CCOCC1</smiles>

20 hours, 52\% yield (using L20 as ligand), known compound ${ }^{2 \mathrm{~b}} ;{ }^{1} \mathrm{H}$ NMR (500 MHz, $\left.\mathrm{CDCl}_{3}\right): \delta 7.02-7.00(\mathrm{~m}, 1 \mathrm{H}), 6.97-6.94(\mathrm{~m}, 1 \mathrm{H}), 6.79-6.76(\mathrm{~m}, 2 \mathrm{H}), 4.12$ (brs, 2H), $3.85(\mathrm{t}, J=4.5 \mathrm{~Hz}, 4 \mathrm{H}), 2.92(\mathrm{t}, J=4.5 \mathrm{~Hz}, 4 \mathrm{H})$.<smiles>Fc1ccccc1N1CCOCC1</smiles>

20 hours, 57\% yield (using L22 as ligand), known compound ${ }^{2 c}$; ${ }^{1} \mathrm{H}$ NMR (500 MHz, $\left.\mathrm{CDCl}_{3}\right): \delta 7.09-7.01(\mathrm{~m}, 2 \mathrm{H}), 6.97-6.93(\mathrm{~m}, 2 \mathrm{H}), 3.88(\mathrm{t}, J=5.0 \mathrm{~Hz}, 4 \mathrm{H}), 3.09(\mathrm{t}, J=$ $5.0 \mathrm{~Hz}, 4 \mathrm{H})$.<smiles></smiles>

20 hours, 62\% yield (using L22 as ligand), known compound ${ }^{2 \mathrm{~d}} ;{ }^{1} \mathrm{H}$ NMR (500 MHz, $\left.\mathrm{CDCl}_{3}\right): \delta 7.38-7.36(\mathrm{~m}, 1 \mathrm{H}), 7.25-7.22(\mathrm{~m}, 1 \mathrm{H}), 7.05-6.97(\mathrm{~m}, 2 \mathrm{H}), 3.88(\mathrm{t}, J=4.5$ $\mathrm{Hz}, 4 \mathrm{H}), 3.06(\mathrm{t}, J=4.5 \mathrm{~Hz}, 4 \mathrm{H})$.<smiles>COc1cccc(N2CCOCC2)c1</smiles>

20 hours, $51 \%$ yield (using L20 as ligand), known compound ${ }^{2 \mathrm{a}} ;{ }^{1} \mathrm{H}$ NMR (500 MHz, $\left.\mathrm{CDCl}_{3}\right): \delta 7.18(\mathrm{t}, J=8.5 \mathrm{~Hz}, 1 \mathrm{H}), 6.54-6.52(\mathrm{~m}, 1 \mathrm{H}), 6.46-6.43(\mathrm{~m}, 2 \mathrm{H}), 3.85(\mathrm{t}, J=$ $5.0 \mathrm{~Hz}, 4 \mathrm{H}), 3.79(\mathrm{~s}, 3 \mathrm{H}), 3.15(\mathrm{t}, J=5.0 \mathrm{~Hz}, 4 \mathrm{H})$. 
<smiles>CSc1cccc(N2CCOCC2)c1</smiles>

20 hours, 89\% yield (using L20 as ligand), known compound ${ }^{2 \mathrm{e}} ;{ }^{1} \mathrm{H}$ NMR (500 MHz, $\left.\mathrm{CDCl}_{3}\right): \delta 7.19(\mathrm{t}, J=8.0 \mathrm{~Hz}, 1 \mathrm{H}), 6.82-6.77(\mathrm{~m}, 2 \mathrm{H}), 6.71-6.67(\mathrm{~m}, 1 \mathrm{H}), 3.85(\mathrm{t}, J=$ $5.0 \mathrm{~Hz}, 4 \mathrm{H}), 3.15(\mathrm{t}, J=5.0 \mathrm{~Hz}, 4 \mathrm{H}), 2.47(\mathrm{~s}, 3 \mathrm{H})$.<smiles>Nc1cccc(N2CCOCC2)c1</smiles>

20 hours, 64\% yield (using L20 as ligand), known compound ${ }^{2 f} ;{ }^{1} \mathrm{H}$ NMR (500 MHz, $\left.\mathrm{CDCl}_{3}\right): \delta 7.07-7.04(\mathrm{~m}, 1 \mathrm{H}), 6.36-6.34(\mathrm{~m}, 1 \mathrm{H}), 6.24-6.23(\mathrm{~m}, 2 \mathrm{H}), 3.84(\mathrm{t}, J=5.0$ $\mathrm{Hz}, 4 \mathrm{H}), 3.63$ (brs, 2H), $3.12(\mathrm{t}, J=5.0 \mathrm{~Hz}, 4 \mathrm{H})$.<smiles>CC(C)=Cc1cccc(N2CCOCC2)c1</smiles>

Colourless oil, 20 hours, 64\% yield (using L22 as ligand); ${ }^{1} \mathrm{H}$ NMR (500 MHz, $\left.\mathrm{CDCl}_{3}\right): \delta 7.25-7.20(\mathrm{~m}, 1 \mathrm{H}), 6.78-6.75(\mathrm{~m}, 3 \mathrm{H}), 6.24(\mathrm{~s}, 1 \mathrm{H}), 3.85(\mathrm{t}, J=5.0 \mathrm{~Hz}$, $4 \mathrm{H}), 3.15(\mathrm{t}, J=5.0 \mathrm{~Hz}, 4 \mathrm{H}), 1.89(\mathrm{~s}, 3 \mathrm{H}), 1.86(\mathrm{~s}, 3 \mathrm{H}) ;{ }^{13} \mathrm{C} \mathrm{NMR}\left(125 \mathrm{MHz}, \mathrm{CDCl}_{3}\right)$ : $\delta 151.1,139.6,135.4,128.7,125.4,120.7,116.3,113.5,66.9,49.5,26.8,19.5$; HRMS-ESI (m/z): $[\mathrm{M}+\mathrm{H}]^{+}$calcd. for $\left[\mathrm{C}_{14} \mathrm{H}_{20} \mathrm{NO}\right]^{+}, 218.1539$; found, 218.1539 .<smiles>Cc1cccc(N2CCOCC2)c1</smiles>

20 hours, 89\% yield (using L22 as ligand), known compound ${ }^{2 c} ;{ }^{1} \mathrm{H}$ NMR (500 MHz, $\left.\mathrm{CDCl}_{3}\right): \delta 7.16(\mathrm{t}, J=7.5 \mathrm{~Hz}, 1 \mathrm{H}), 6.73-6.70(\mathrm{~m}, 3 \mathrm{H}), 3.84(\mathrm{t}, J=5.0 \mathrm{~Hz}, 4 \mathrm{H}), 3.13(\mathrm{t}$, $J=5.0 \mathrm{~Hz}, 4 \mathrm{H}), 2.32(\mathrm{~s}, 3 \mathrm{H})$.<smiles>c1ccc(Cc2ccccc2N2CCOCC2)cc1</smiles>

White cream, 20 hours, 75\% yield (using $\mathbf{L 2 2}$ as ligand); ${ }^{1} \mathrm{H}$ NMR (500 $\mathrm{MHz}, \mathrm{CDCl}_{3}$ ): ס 7.27-7.24 (m, 2H), 7.19-7.16 (m, 4H), 6.74-6.70 (m, 3H), $3.93(\mathrm{~s}, 2 \mathrm{H}), 3.81(\mathrm{t}, J=$ $5.0 \mathrm{~Hz}, 4 \mathrm{H}), 3.10(\mathrm{t}, J=5.0 \mathrm{~Hz}, 4 \mathrm{H}) ;{ }^{13} \mathrm{C} \mathrm{NMR}\left(125 \mathrm{MHz}, \mathrm{CDCl}_{3}\right): \delta 151.4,142.0$, 141.0, 129.2, 128.8, 128.4, 126.0, 120.8, 116.4, 113.3, 66.9, 49.3, 42.2; HRMS-ESI 
$(\mathrm{m} / \mathrm{z}):[\mathrm{M}+\mathrm{H}]^{+}$calcd. for $\left[\mathrm{C}_{17} \mathrm{H}_{20} \mathrm{NO}\right]^{+}, 254.1539$; found, 254.1535 .<smiles>CC(O)(O)c1cccc(N2CCOCC2)c1</smiles>

Colourless oil, 20 hours, 82\% yield (using L22 as ligand); ${ }^{1} \mathrm{H}$ NMR (500 MHz, $\left.\mathrm{CDCl}_{3}\right): \delta 7.24(\mathrm{t}, J=8.0 \mathrm{~Hz}, 1 \mathrm{H}), 7.12(\mathrm{t}, J=2.0 \mathrm{~Hz}, 1 \mathrm{H}), 6.96(\mathrm{~d}, J=7.5 \mathrm{~Hz}, 1 \mathrm{H})$, 6.80-6.78 (m, 1H), 6.24-6.23 (m, 2H), $3.85(\mathrm{t}, J=5.0 \mathrm{~Hz}, 4 \mathrm{H}), 3.17(\mathrm{t}, J=5.0 \mathrm{~Hz}$, $4 \mathrm{H}), 1.99$ (brs, $1 \mathrm{H}), 1.56$ (s, 6H); ${ }^{13} \mathrm{C} \mathrm{NMR}\left(125 \mathrm{MHz}, \mathrm{CDCl}_{3}\right): \delta 151.2,150.3,128.9$, 116.3, 113.9, 112.1, 72.6, 66.9, 49.5, 31.7; HRMS-ESI (m/z): $[\mathrm{M}+\mathrm{H}]^{+}$calcd. for $\left[\mathrm{C}_{13} \mathrm{H}_{20} \mathrm{NO}_{2}\right]^{+}, 222.1489$; found, 222.1483.<smiles>c1cc(CCN2CCOCC2)cc(N2CCOCC2)c1</smiles>

$2 m$

White cream, 20 hours, 82\% yield (using $\mathbf{L 2 2}$ as ligand); ${ }^{1} \mathrm{H}$ NMR (500 MHz, $\mathrm{CDCl}_{3}$ ): $\delta 7.22-7.18(\mathrm{~m}, 1 \mathrm{H}), 6.77-6.73(\mathrm{~m}, 3 \mathrm{H}), 3.86(\mathrm{t}, J=5.0 \mathrm{~Hz}, 4 \mathrm{H}), 3.76$ (brs, 4H), 3.15 $(\mathrm{t}, J=5.0 \mathrm{~Hz}, 4 \mathrm{H}), 2.79(\mathrm{t}, J=7.5 \mathrm{~Hz}, 2 \mathrm{H}), 2.62(\mathrm{t}, J=7.5 \mathrm{~Hz}, 2 \mathrm{H}), 2.55(\mathrm{~s}, 4 \mathrm{H}) ;{ }^{13} \mathrm{C}$ NMR $\left(125 \mathrm{MHz}, \mathrm{CDCl}_{3}\right): \delta 151.5,141.1,129.2,120.4,116.2,113.5,66.92,66.88$, 60.8, 53.6, 49.4, 33.6; HRMS-ESI (m/z): $[\mathrm{M}+\mathrm{H}]^{+}$calcd. for $\left[\mathrm{C}_{16} \mathrm{H}_{25} \mathrm{~N}_{2} \mathrm{O}_{2}\right]^{+}, 277.1911$; found, 277.1917.<smiles>COc1ccc(N2CCOCC2)cc1</smiles>

20 hours, $10 \mathrm{~mol} \%\left[\mathrm{Ru}(\text { cymene }) \mathrm{Cl}_{2}\right]_{2} ; 60 \%$ yield (using $\mathbf{L 2 0}$ as ligand), known compound $^{2 \mathrm{a}} ;{ }^{1} \mathrm{H}$ NMR $\left(500 \mathrm{MHz}, \mathrm{CDCl}_{3}\right): \delta 6.90-6.83(\mathrm{~m}, 4 \mathrm{H}), 3.85(\mathrm{t}, J=5.0 \mathrm{~Hz}$, $4 \mathrm{H}), 3.77(\mathrm{~s}, 3 \mathrm{H}), 3.05(\mathrm{t}, J=5.0 \mathrm{~Hz}, 4 \mathrm{H})$.<smiles>Nc1ccc(N2CCOCC2)cc1</smiles>

48 hours, $5 \mathrm{~mol} \%\left[\mathrm{Ru}\left(\text { cymene) } \mathrm{Cl}_{2}\right]_{2} ; 53 \%\right.$ yield (using $\mathbf{L 2 0}$ as ligand), known compound ${ }^{2 \mathrm{~g}}$; ${ }^{1} \mathrm{H}$ NMR $\left(500 \mathrm{MHz}, \mathrm{CDCl}_{3}\right): \delta 6.81-6.78(\mathrm{~m}, 2 \mathrm{H}), 6.67-6.64(\mathrm{~m}, 2 \mathrm{H})$, $3.84(\mathrm{t}, J=4.5 \mathrm{~Hz}, 4 \mathrm{H}), 3.44$ (brs, $2 \mathrm{H}), 3.10(\mathrm{t}, J=5.0 \mathrm{~Hz}, 4 \mathrm{H})$.<smiles>c1ccc(-c2ccc(N3CCOCC3)cc2)cc1</smiles> 
20 hours, 89\% yield (using L22 as ligand), known compound ${ }^{2 \mathrm{~h}} ;{ }^{1} \mathrm{H}$ NMR (500 MHz, $\left.\mathrm{CDCl}_{3}\right): \delta 7.57-7.52(\mathrm{~m}, 4 \mathrm{H}), 7.41(\mathrm{t}, J=7.5 \mathrm{~Hz}, 2 \mathrm{H}), 7.29(\mathrm{t}, J=7.5 \mathrm{~Hz}, 1 \mathrm{H}), 6.99$ $(\mathrm{d}, J=8.5 \mathrm{~Hz}, 2 \mathrm{H}), 3.89(\mathrm{t}, J=5.0 \mathrm{~Hz}, 4 \mathrm{H}), 3.21(\mathrm{t}, J=5.0 \mathrm{~Hz}, 4 \mathrm{H})$.<smiles>CCCCOc1ccc(N2CCOCC2)cc1</smiles>

White cream, 20 hours, $45 \%$ yield (using $\mathbf{L 2 2}$ as ligand); ${ }^{1} \mathrm{H} \mathrm{NMR}\left(500 \mathrm{MHz}, \mathrm{CDCl}_{3}\right)$ : $\delta 7.23(\mathrm{~d}, J=8.5 \mathrm{~Hz}, 2 \mathrm{H}), 6.88(\mathrm{~d}, J=8.5 \mathrm{~Hz}, 2 \mathrm{H}), 4.66(\mathrm{~s}, 2 \mathrm{H}), 3.86(\mathrm{t}, J=5.0 \mathrm{~Hz}$, $4 \mathrm{H}), 3.14(\mathrm{t}, J=5.0 \mathrm{~Hz}, 4 \mathrm{H}), 0.93(\mathrm{~s}, 9 \mathrm{H}), 0.08(\mathrm{~s}, 6 \mathrm{H}) ;{ }^{13} \mathrm{C} \mathrm{NMR}\left(125 \mathrm{MHz}, \mathrm{CDCl}_{3}\right)$ : $\delta$ 150.4, 133.0, 127.3, 115.6, 66.9, 64.7, 49.6, 26.0, 18.4, -5.2; HRMS-ESI (m/z): $[\mathrm{M}+\mathrm{H}]^{+}$calcd. for $\left[\mathrm{C}_{17} \mathrm{H}_{30} \mathrm{NO}_{2} \mathrm{Si}\right]^{+}, 308.2040$; found, 308.2033.<smiles>CC(C)NCc1ccc(N2CCOCC2)cc1</smiles>

White cream, 20 hours, $80 \%$ yield (using $\mathbf{L 2 2}$ as ligand); ${ }^{1} \mathrm{H}$ NMR (500 MHz, $\mathrm{CDCl}_{3}$ ): $\delta 7.20(\mathrm{~d}, J=8.5 \mathrm{~Hz}, 2 \mathrm{H}), 6.87(\mathrm{~d}, J=8.5 \mathrm{~Hz}, 2 \mathrm{H}), 5.69$ (brs, $1 \mathrm{H}), 4.34$ (d, $J=5.5 \mathrm{~Hz}$, $2 \mathrm{H}), 3.86(\mathrm{t}, J=5.0 \mathrm{~Hz}, 4 \mathrm{H}), 3.14(\mathrm{t}, J=5.0 \mathrm{~Hz}, 4 \mathrm{H}), 2.00(\mathrm{~s}, 3 \mathrm{H}) ;{ }^{13} \mathrm{C}$ NMR $(125$ $\left.\mathrm{MHz}, \mathrm{CDCl}_{3}\right): \delta 169.7,150.8,129.5,129.0,115.8,66.8,49.3,43.2,23.3$; HRMS-ESI $(\mathrm{m} / \mathrm{z}):[\mathrm{M}+\mathrm{H}]^{+}$calcd. for $\left[\mathrm{C}_{13} \mathrm{H}_{19} \mathrm{~N}_{2} \mathrm{O}_{2}\right]^{+}, 235.1436$; found, 235.1441 .<smiles>[Mg]NCc1ccc(N2CCOCC2)cc1</smiles>

White cream, 48 hours, 50\% yield (using $\mathbf{L 2 2}$ as ligand); ${ }^{1} \mathrm{H}$ NMR (500 $\mathrm{MHz}, \mathrm{CDCl}_{3}$ ): $\delta 7.72(\mathrm{~d}, J=8.0 \mathrm{~Hz}, 2 \mathrm{H}), 7.31(\mathrm{~d}, J=8.5 \mathrm{~Hz}, 2 \mathrm{H}), 7.09(\mathrm{~d}, J=8.5 \mathrm{~Hz}, 2 \mathrm{H}), 6.80(\mathrm{~d}$, $J=8.5 \mathrm{~Hz}, 2 \mathrm{H}), 4.63(\mathrm{t}, J=5.5 \mathrm{~Hz}, 1 \mathrm{H}), 4.03(\mathrm{~d}, J=5.5 \mathrm{~Hz}, 2 \mathrm{H}), 3.84(\mathrm{t}, J=5.0 \mathrm{~Hz}$, $4 \mathrm{H}), 3.11(\mathrm{t}, J=5.0 \mathrm{~Hz}, 4 \mathrm{H}), 2.44(\mathrm{~s}, 9 \mathrm{H}) ;{ }^{13} \mathrm{C} \mathrm{NMR}\left(125 \mathrm{MHz}, \mathrm{CDCl}_{3}\right): \delta 151.0$, $143.4,136.9,129.7,129.0,127.3,127.2,115.6,66.8,49.1,46.8,21.5$; HRMS-ESI $(\mathrm{m} / \mathrm{z}):[\mathrm{M}+\mathrm{H}]^{+}$calcd. for $\left[\mathrm{C}_{18} \mathrm{H}_{23} \mathrm{~N}_{2} \mathrm{O}_{2} \mathrm{~S}\right]^{+}, 347.1424$; found, 347.1425 .<smiles>Brc1ccccc1</smiles>

20 hours, 37\% yield (using L22 as ligand), known compound ${ }^{2 \mathrm{~h}} ;{ }^{1} \mathrm{H}$ NMR (500 MHz, $\left.\mathrm{CDCl}_{3}\right): \delta 7.72(\mathrm{~d}, J=9.0 \mathrm{~Hz}, 2 \mathrm{H}), 6.88(\mathrm{~d}, J=8.5 \mathrm{~Hz}, 2 \mathrm{H}), 3.85(\mathrm{t}, J=5.0 \mathrm{~Hz}, 4 \mathrm{H})$, $3.22(\mathrm{t}, J=5.0 \mathrm{~Hz}, 4 \mathrm{H}), 1.33(\mathrm{~s}, 12 \mathrm{H})$. 
<smiles>O=C(Nc1ccc(N2CCOCC2)cc1)c1ccccc1</smiles>

20 hours, 46\% yield (using L20 as ligand), known compound ${ }^{2 \mathrm{i}} ;{ }^{1} \mathrm{H}$ NMR (500 MHz, $\left.\mathrm{CDCl}_{3}\right): \delta 7.26(\mathrm{~d}, J=8.5 \mathrm{~Hz}, 2 \mathrm{H}), 6.87(\mathrm{~d}, J=9.0 \mathrm{~Hz}, 2 \mathrm{H}), 6.38($ br s, $1 \mathrm{H}), 3.86(\mathrm{t}, J$ $=5.0 \mathrm{~Hz}, 4 \mathrm{H}), 3.09(\mathrm{t}, J=5.0 \mathrm{~Hz}, 4 \mathrm{H}), 1.51(\mathrm{~s}, 9 \mathrm{H})$.<smiles>Cc1ccc(N2CCOCC2)cc1[N+](=O)[O-]</smiles>

48 hours, $5 \mathrm{~mol} \%\left[\mathrm{Ru}(\text { cymene }) \mathrm{Cl}_{2}\right]_{2}, 80 \%$ yield (using $\mathbf{L 2 0}$ as ligand), known compound ${ }^{2 \mathrm{~d}}$; ${ }^{1} \mathrm{H}$ NMR (500 MHz, $\left.\mathrm{CDCl}_{3}\right): \delta 7.03(\mathrm{~d}, J=8.5 \mathrm{~Hz}, 1 \mathrm{H}), 6.73(\mathrm{~d}, J=2.0$ $\mathrm{Hz}, 1 \mathrm{H}), 6.67(\mathrm{dd}, J=8.5 \mathrm{~Hz}, J=2.0 \mathrm{~Hz}, 1 \mathrm{H}), 3.84(\mathrm{t}, J=5.0 \mathrm{~Hz}, 4 \mathrm{H}), 3.09(\mathrm{t}, J=5.0$ $\mathrm{Hz}, 4 \mathrm{H}), 2.23$ (s, 3H), 2.18 (s, 3H).<smiles>Nc1ccc(F)c(N2CCOCC2)c1</smiles>

White cream, 20 hours, 93\% yield (using $\mathbf{L 2 2}$ as ligand); ${ }^{1} \mathrm{H} \mathrm{NMR}\left(500 \mathrm{MHz}, \mathrm{CDCl}_{3}\right.$ ): $\delta$ 6.83-6.79 (m, 1H), 6.26-6.21 (m, 2H), $3.85(\mathrm{t}, J=4.5 \mathrm{~Hz}, 4 \mathrm{H}), 3.53$ (brs, 2H), 3.04 $(\mathrm{t}, J=4.5 \mathrm{~Hz}, 4 \mathrm{H}) ;{ }^{13} \mathrm{C} \mathrm{NMR}\left(125 \mathrm{MHz}, \mathrm{CDCl}_{3}\right): \delta 149.3(\mathrm{~d}, J=234.5 \mathrm{~Hz}), 142.9(\mathrm{~d}$, $J=1.4 \mathrm{~Hz}), 140.3(\mathrm{~d}, J=9.4 \mathrm{~Hz}), 116.4(\mathrm{~d}, J=21.8 \mathrm{~Hz}), 108.3(\mathrm{~d}, J=8.0 \mathrm{~Hz}), 105.7$ $(\mathrm{d}, J=2.0 \mathrm{~Hz}), 66.9,50.77,50.74 ;{ }^{19} \mathrm{~F} \mathrm{NMR}\left(470 \mathrm{MHz}, \mathrm{CDCl}_{3}\right):-135.94--136.00$ (m); HRMS-ESI $(\mathrm{m} / \mathrm{z})$ : $[\mathrm{M}+\mathrm{H}]^{+}$calcd. for $\left[\mathrm{C}_{10} \mathrm{H}_{14} \mathrm{~N}_{2} \mathrm{OF}\right]^{+}, 197.1085$; found, 197.1075.<smiles>Oc1ccc(F)c(N2CCOCC2)c1</smiles>

White cream, 20 hours, 80\% yield (using $\mathbf{L 2 2}$ as ligand); ${ }^{1} \mathrm{H}$ NMR (500 $\mathrm{MHz}, \mathrm{CDCl}_{3}$ ): $\delta$ 6.90-6.86 (m, 1H), 6.43-6.41 (m, 1H), 6.37-6.34 (m, 1H), 5.29 (brs, 1H), 3.88 (t, $J$ $=4.5 \mathrm{~Hz}, 4 \mathrm{H}), 3.06(\mathrm{t}, J=4.5 \mathrm{~Hz}, 4 \mathrm{H}) ;{ }^{13} \mathrm{C} \mathrm{NMR}\left(125 \mathrm{MHz}, \mathrm{CDCl}_{3}\right): \delta 152.2(\mathrm{~d}, J=$ $2.0 \mathrm{~Hz}), 150.1(\mathrm{~d}, J=237.3 \mathrm{~Hz}), 140.5(\mathrm{~d}, J=10.3 \mathrm{~Hz}), 116.5(\mathrm{~d}, J=21.9 \mathrm{~Hz}), 108.2$ $(\mathrm{d}, J=7.4 \mathrm{~Hz}), 106.0(\mathrm{~d}, J=2.8 \mathrm{~Hz}), 66.9,50.70,50.67 ;{ }^{19} \mathrm{~F} \mathrm{NMR}\left(470 \mathrm{MHz}, \mathrm{CDCl}_{3}\right)$ : -133.43 - -133.47 (m); HRMS-ESI $(\mathrm{m} / \mathrm{z})$ : $[\mathrm{M}+\mathrm{H}]^{+}$calcd. for $\left[\mathrm{C}_{10} \mathrm{H}_{13} \mathrm{NO}_{2} \mathrm{~F}\right]^{+}$, 198.0925; found, 198.0919. 
<smiles>Nc1cccc(N2CCOCC2)c1Cl</smiles>

White cream, 48 hours, $45 \%$ yield (using $\mathbf{L 2 2}$ as ligand); ${ }^{1} \mathrm{H}$ NMR (500 $\mathrm{MHz}, \mathrm{CDCl}_{3}$ ): א 7.04-7.01 (m, 1H), 6.53-6.51 (m, 1H), 6.47-6.45 (m, 1H), 4.10 (brs, 2H), 3.87 (t, $J$ $=4.5 \mathrm{~Hz}, 4 \mathrm{H}), 3.01(\mathrm{t}, J=4.5 \mathrm{~Hz}, 4 \mathrm{H}),{ }^{13} \mathrm{C} \mathrm{NMR}\left(125 \mathrm{MHz}, \mathrm{CDCl}_{3}\right): \delta 149.9,144.3$, 127.3, 113.9, 110.9, 109.7, 67.2, 51.8; HRMS-ESI $(\mathrm{m} / \mathrm{z}):[\mathrm{M}+\mathrm{H}]^{+}$calcd. for $\left[\mathrm{C}_{10} \mathrm{H}_{14} \mathrm{~N}_{2} \mathrm{OCl}\right]^{+}, 213.0789$; found, 213.0780.<smiles>O=C(c1cccc(N2CCOCC2)c1)c1cc(F)cc(C(F)(F)F)c1</smiles>

Colourless oil, 48 hours, 60\% yield (using L22 as ligand); ${ }^{1} \mathrm{H}$ NMR (600 MHz, $\left.\mathrm{CDCl}_{3}\right): \delta 7.27(\mathrm{~s}, 1 \mathrm{H}), 7.23(\mathrm{t}, J=7.8 \mathrm{~Hz}, 1 \mathrm{H}), 7.15(\mathrm{~d}, J=8.4 \mathrm{~Hz}, 1 \mathrm{H}), 7.04(\mathrm{~d}, J=$ $9.6 \mathrm{~Hz}, 1 \mathrm{H}), 6.81-6.79(\mathrm{~m}, 1 \mathrm{H}), 6.72(\mathrm{~s}, 1 \mathrm{H}), 6.68(\mathrm{~d}, J=7.8 \mathrm{~Hz}, 1 \mathrm{H}), 3.97(\mathrm{~s}, 2 \mathrm{H})$, $3.84(\mathrm{t}, J=4.8 \mathrm{~Hz}, 4 \mathrm{H}), 3.14(\mathrm{t}, J=4.8 \mathrm{~Hz}, 4 \mathrm{H}) ;{ }^{13} \mathrm{C} \mathrm{NMR}\left(150 \mathrm{MHz}, \mathrm{CDCl}_{3}\right): \delta$ $162.5(\mathrm{~d}, J=246.8 \mathrm{~Hz}), 151.7,145.0(\mathrm{~d}, J=8.0 \mathrm{~Hz}), 140.0,132.7-131.9(\mathrm{~m}), 129.6$, 126.0-120.6 (m), 121.4-121.3 (m), 120.6, 119.2 (d, $J=21.9 \mathrm{~Hz}), 116.2,113.9$, 110.7-110.4 (m), 66.8, 49.2, 41.8; ${ }^{19} \mathrm{~F}$ NMR (470 MHz, $\left.\mathrm{CDCl}_{3}\right)$ : -62.6, -111.16 $-111.19(\mathrm{~m})$; HRMS-ESI (m/z): $[\mathrm{M}+\mathrm{H}]^{+}$calcd. for $\left[\mathrm{C}_{18} \mathrm{H}_{18} \mathrm{NOF}_{4}\right]^{+}, 340.1319$; found, 340.1325 .

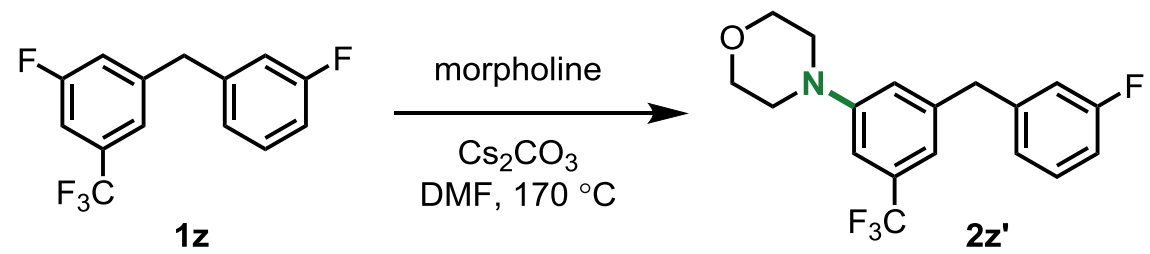

Aryl fluoride $\mathbf{1 z}(0.2 \mathrm{mmol})$ was mixed with morpholine $(0.6 \mathrm{mmol}), \mathrm{Cs}_{2} \mathrm{CO}_{3}(0.4$ mmol) and DMF $(0.2 \mathrm{~mL})$ under $\mathrm{N}_{2}$ atmosphere. The mixture was stirred at $170^{\circ} \mathrm{C}$ for 48 hours (the produced dimethylamine was removed under vaccum with the proceeding reaction and another $0.6 \mathrm{mmol}$ morpholine was added at 18th hour). The reaction was cooled to room temperature, $\mathrm{H}_{2} \mathrm{O}(5 \mathrm{~mL})$ was added and extracted with $\mathrm{Et}_{2} \mathrm{O}(15 \mathrm{~mL} \times 5)$. The organic phase was separated, dried by $\mathrm{Na}_{2} \mathrm{SO}_{4}$ and concentrated. The crude product was further purified by chromatography on silica gel (ethyl acetate/ petroleum ether $=1 / 10$ ), affording $\mathbf{2 z}$ ' as a colourless oil in $58 \%$ yield. ${ }^{1} \mathrm{H}$ NMR (500 MHz, $\mathrm{CDCl}_{3}$ ): $\delta$ 7.28-7.24 (m, 1H, containing $\mathrm{CDCl}_{3}$ peak), 6.97-6.84 $(\mathrm{m}, 6 \mathrm{H}), 3.97(\mathrm{~s}, 2 \mathrm{H}), 3.85(\mathrm{t}, J=5.0 \mathrm{~Hz}, 4 \mathrm{H}), 3.17(\mathrm{t}, J=5.0 \mathrm{~Hz}, 4 \mathrm{H}) ;{ }^{13} \mathrm{C} \mathrm{NMR}(125$ $\left.\mathrm{MHz}, \mathrm{CDCl}_{3}\right): \delta 163.0(\mathrm{~d}, J=245 \mathrm{~Hz}), 151.7,142.6(\mathrm{~d}, J=7.8 \mathrm{~Hz}), 142.1$, 132.1-131.3 (m), 130.0 (d, $J=9.4 \mathrm{~Hz}), 127.4-120.9(\mathrm{~m}), 124.5(\mathrm{~d}, J=3.5 \mathrm{~Hz}), 118.9$, 116.9-116.8 (m), $115.7(\mathrm{~d}, J=20.9 \mathrm{~Hz}), 113.3(\mathrm{~d}, J=21.3 \mathrm{~Hz}), 110.1-110.0(\mathrm{~m}), 66.7$, 48.8, 41.7; ${ }^{19} \mathrm{~F}$ NMR (470 MHz, $\mathrm{CDCl}_{3}$ ): -62.7, -113.12 - -113.16 (m); HRMS-ESI $(\mathrm{m} / \mathrm{z}):[\mathrm{M}+\mathrm{H}]^{+}$calcd. for $\left[\mathrm{C}_{18} \mathrm{H}_{18} \mathrm{NOF}_{4}\right]^{+}, 340.1319$; found, 340.1324 . 
<smiles>Nc1ccc(F)c(N2CCCCC2)c1</smiles>

White cream, 20 hours, 95\% yield (using $\mathbf{L 2 2}$ as ligand); ${ }^{1} \mathrm{H}$ NMR (500 $\mathrm{MHz}, \mathrm{CDCl}_{3}$ ): $\delta$ 6.80-6.76 (m, 1H), 6.28-6.16 (m, 2H), 3.44 (brs, 2H), $2.96(\mathrm{t}, J=5.5 \mathrm{~Hz}, 4 \mathrm{H})$, $1.73-1.69(\mathrm{~m}, 4 \mathrm{H}), 1.57-1.52(\mathrm{~m}, 2 \mathrm{H}) ;{ }^{13} \mathrm{C} \mathrm{NMR}\left(125 \mathrm{MHz}, \mathrm{CDCl}_{3}\right): \delta 149.4(\mathrm{~d}, J=$ $234.6 \mathrm{~Hz}), 142.7,141.7$ (d, $J=8.3 \mathrm{~Hz}), 116.1(\mathrm{~d}, J=22.0 \mathrm{~Hz}), 107.7$ (d, $J=7.9 \mathrm{~Hz})$, $106.3(\mathrm{~d}, J=1.8 \mathrm{~Hz}), 51.93,51.89,26.1,24.2 ;{ }^{19} \mathrm{~F} \mathrm{NMR}\left(470 \mathrm{MHz}, \mathrm{CDCl}_{3}\right):-135.56$ $--135.60(\mathrm{~m})$; HRMS-ESI (m/z): $[\mathrm{M}+\mathrm{H}]^{+}$calcd. for $\left[\mathrm{C}_{11} \mathrm{H}_{16} \mathrm{~N}_{2} \mathrm{~F}\right]^{+}, 195.1292$; found, 195.1290.<smiles>Nc1ccc(F)c(N2CCC(c3ccccc3)CC2)c1</smiles>

White cream, 20 hours, 64\% yield (using L6 as ligand); ${ }^{1} \mathrm{H} \mathrm{NMR}\left(500 \mathrm{MHz}, \mathrm{CDCl}_{3}\right)$ : $\delta$ 7.33-7.19 (m, 5H), 6.83-6.79 (m, 1H), 6.33-6.31 (m, 1H), 6.22-6.19 (m, 1H), 3.55-3.53 (m, 2H), 3.47 (brs, 2H), 2.76-2.71 (m, 2H), 2.65-2.58 (m, 1H), 2.03-1.90 $(\mathrm{m}, 4 \mathrm{H}) ;{ }^{13} \mathrm{C}$ NMR $\left(125 \mathrm{MHz}, \mathrm{CDCl}_{3}\right): \delta 149.5(\mathrm{~d}, J=235.0 \mathrm{~Hz}), 146.1,142.7,141.2$ $(\mathrm{d}, J=9.8 \mathrm{~Hz}), 128.4,126.8,126.2,116.2(\mathrm{~d}, J=21.9 \mathrm{~Hz}), 107.9(\mathrm{~d}, J=6.9 \mathrm{~Hz})$, $106.4(\mathrm{~d}, J=1.4 \mathrm{~Hz}), 51.69,51.66,42.4,33.5 ;{ }^{19} \mathrm{~F} \mathrm{NMR}\left(470 \mathrm{MHz}, \mathrm{CDCl}_{3}\right)$ : -135.5; HRMS-ESI (m/z): $[\mathrm{M}+\mathrm{H}]^{+}$calcd. for $\left[\mathrm{C}_{17} \mathrm{H}_{20} \mathrm{~N}_{2} \mathrm{~F}\right]^{+}, 271.1605$; found, 271.1599 .<smiles>CC(C)(C)NC1CCN(c2cc(N)ccc2F)CC1</smiles>

White cream, 20 hours, 59\% yield (using $\mathbf{L 2 2}$ as ligand); ${ }^{1} \mathrm{H}$ NMR (500 $\mathrm{MHz}, \mathrm{CDCl}_{3}$ ): $\delta 6.82-6.78(\mathrm{~m}, 1 \mathrm{H}), 6.29-6.20(\mathrm{~m}, 2 \mathrm{H}), 4.48(\mathrm{~s}, 1 \mathrm{H}), 3.58-3.36(\mathrm{~m}, 5 \mathrm{H}), 2.73(\mathrm{t}, J=$ $11.0 \mathrm{~Hz}, 2 \mathrm{H}), 2.05-2.02(\mathrm{~m}, 2 \mathrm{H}), 1.63-1.56(\mathrm{~m}, 2 \mathrm{H}), 1.46(\mathrm{~s}, 9 \mathrm{H}) ;{ }^{13} \mathrm{C}$ NMR $(125$ $\left.\mathrm{MHz}, \mathrm{CDCl}_{3}\right): \delta 155.2,149.4(\mathrm{~d}, J=234.9 \mathrm{~Hz}), 142.8(\mathrm{~d}, J=1.9 \mathrm{~Hz}), 140.7,116.3(\mathrm{~d}$, $J=22.1 \mathrm{~Hz}), 108.2(\mathrm{~d}, J=7.5 \mathrm{~Hz}), 106.4$ (d, $J=2.1 \mathrm{~Hz}), 79.3,49.9,47.7,32.8,28.4$; ${ }^{19} \mathrm{~F}$ NMR (470 MHz, $\left.\mathrm{CDCl}_{3}\right):-135.7$; HRMS-ESI $(\mathrm{m} / \mathrm{z}):[\mathrm{M}+\mathrm{H}]^{+}$calcd. for $\left[\mathrm{C}_{16} \mathrm{H}_{25} \mathrm{~N}_{3} \mathrm{O}_{2} \mathrm{~F}\right]^{+}$, 310.1925; found, 310.1919.<smiles>Nc1ccc(F)c(N2CCc3ccccc3C2)c1</smiles>

White cream, 20 hours, 98\% yield (using $\mathbf{L 2 2}$ as ligand); ${ }^{1} \mathrm{H}$ NMR (500 $\mathrm{MHz}, \mathrm{CDCl}_{3}$ ): d 7.17-7.08 (m, 4H), 6.85-6.81 (m, 1H), 6.33-6.31 (m, 1H), 6.22-6.19 (m, 1H), 4.26 (s, 2H), $3.48(\mathrm{brs}, 2 \mathrm{H}), 3.41(\mathrm{t}, J=6.0 \mathrm{~Hz}, 2 \mathrm{H}), 2.96(\mathrm{t}, J=6.0 \mathrm{~Hz}, 2 \mathrm{H}) ;{ }^{13} \mathrm{C} \mathrm{NMR}$ 
$\left(125 \mathrm{MHz}, \mathrm{CDCl}_{3}\right): \delta 149.4(\mathrm{~d}, J=234.8 \mathrm{~Hz}), 142.8(\mathrm{~d}, J=1.9 \mathrm{~Hz}), 140.1(\mathrm{~d}, J=8.5$ $\mathrm{Hz}), 134.42,134.41,128.9,126.3,126.2,125.8,116.4$ (d, $J=20.6 \mathrm{~Hz}), 108.0$ (d, $J=$ $7.5 \mathrm{~Hz}), 106.5(\mathrm{~d}, J=2.3 \mathrm{~Hz}), 52.43,52.41,48.82,48.78,28.8 ;{ }^{19} \mathrm{~F}$ NMR $(470 \mathrm{MHz}$, $\left.\mathrm{CDCl}_{3}\right)$ : $-135.43--135.46(\mathrm{~m})$; HRMS-ESI $(\mathrm{m} / \mathrm{z})$ : $[\mathrm{M}+\mathrm{H}]^{+}$calcd. for $\left[\mathrm{C}_{15} \mathrm{H}_{16} \mathrm{~N}_{2} \mathrm{~F}\right]^{+}$, 243.1292; found, 243.1291.<smiles>CN1CCN(c2cc(N)ccc2F)CC1</smiles>

White cream, 20 hours, $71 \%$ yield (using $\mathbf{L 2 2}$ as ligand); ${ }^{1} \mathrm{H}$ NMR (500 MHz, $\mathrm{CDCl}_{3}$ ): $\delta 6.82-6.78(\mathrm{~m}, 1 \mathrm{H}), 6.28-6.20(\mathrm{~m}, 2 \mathrm{H}), 3.57$ (brs, 2H), $3.10(\mathrm{~s}, 4 \mathrm{H}), 2.63(\mathrm{~s}$, 4H), $2.37(\mathrm{~s}, 3 \mathrm{H}),{ }^{13} \mathrm{C} \mathrm{NMR}\left(125 \mathrm{MHz}, \mathrm{CDCl}_{3}\right): \delta 149.3(\mathrm{~d}, J=235.3 \mathrm{~Hz}), 142.8(\mathrm{~d}, J$ $=2.5 \mathrm{~Hz}), 140.3(\mathrm{~d}, J=10.5 \mathrm{~Hz}), 116.3(\mathrm{~d}, J=21.9 \mathrm{~Hz}), 108.2(\mathrm{~d}, J=7.6 \mathrm{~Hz}), 106.0$ $(\mathrm{d}, J=2.1 \mathrm{~Hz}), 55.0,50.11,50.09,45.9 ;{ }^{19} \mathrm{~F} \mathrm{NMR}\left(470 \mathrm{MHz}, \mathrm{CDCl}_{3}\right):-135.72-$ -135.76 (m); HRMS-ESI (m/z): $[\mathrm{M}+\mathrm{H}]^{+}$calcd. for $\left[\mathrm{C}_{11} \mathrm{H}_{17} \mathrm{~N}_{3} \mathrm{~F}\right]^{+}, 210.1401$; found, 210.1410 .<smiles>Nc1ccc(F)c(N2CCCC2)c1</smiles>

White cream, 20 hours, 50\% yield (using $\mathbf{L 2 2}$ as ligand); ${ }^{1} \mathrm{H} \mathrm{NMR}\left(500 \mathrm{MHz}, \mathrm{CDCl}_{3}\right)$ : $\delta$ 6.78-6.74 (m, 1H), 6.01-5.94 (m, 2H), 3.46 (brs, 2H), 3.35-3.32 (m, 4H), 1.93-1.91 $(\mathrm{m}, 4 \mathrm{H}) ;{ }^{13} \mathrm{C}$ NMR $\left(125 \mathrm{MHz}, \mathrm{CDCl}_{3}\right): \delta 146.3(\mathrm{~d}, J=230.3 \mathrm{~Hz}), 142.8(\mathrm{~d}, J=2.1$ $\mathrm{Hz}), 137.8$ (d, $J=10.9 \mathrm{~Hz}), 116.3(\mathrm{~d}, J=22.0 \mathrm{~Hz}), 103.3$ (d, $J=7.6 \mathrm{~Hz}), 102.4$ (d, $J=$ $3.5 \mathrm{~Hz}), 49.7,49.6,25.14,25.13 ;{ }^{19} \mathrm{~F} \mathrm{NMR}\left(470 \mathrm{MHz}, \mathrm{CDCl}_{3}\right):-139.41$ - -139.45 (m); HRMS-ESI (m/z): $[\mathrm{M}+\mathrm{H}]^{+}$calcd. for $\left[\mathrm{C}_{10} \mathrm{H}_{14} \mathrm{~N}_{2} \mathrm{~F}\right]^{+}, 181.1136$; found, 181.1138 .<smiles>Nc1ccc(F)c(N2CCCCCC2)c1</smiles>

White cream, 20 hours, 98\% yield (using $\mathbf{L 2 2}$ as ligand); ${ }^{1} \mathrm{H}$ NMR (500 $\mathrm{MHz}, \mathrm{CDCl}_{3}$ ): $\delta 6.77-6.73(\mathrm{~m}, 1 \mathrm{H}), 6.18-6.16(\mathrm{~m}, 1 \mathrm{H}), 6.02-5.99(\mathrm{~m}, 1 \mathrm{H}), 3.43$ (brs, 2H), 3.31 (t, $J$ $=5.5 \mathrm{~Hz}, 4 \mathrm{H}), 1.79(\mathrm{~s}, 4 \mathrm{H}), 1.62-1.59(\mathrm{~m}, 4 \mathrm{H}) ;{ }^{13} \mathrm{C} \mathrm{NMR}\left(125 \mathrm{MHz}, \mathrm{CDCl}_{3}\right): \delta 147.5$ $(\mathrm{d}, J=231.6 \mathrm{~Hz}), 142.6(\mathrm{~d}, J=2.1 \mathrm{~Hz}), 140.4(\mathrm{~d}, J=8.9 \mathrm{~Hz}), 116.6(\mathrm{~d}, J=23.0 \mathrm{~Hz})$, $104.4(\mathrm{~d}, J=7.1 \mathrm{~Hz}), 104.0$ (d, $J=3.9 \mathrm{~Hz}), 51.86,51.83,29.1,27.3 ;{ }^{19} \mathrm{~F}$ NMR (470 $\left.\mathrm{MHz}, \mathrm{CDCl}_{3}\right)$ : $-135.94--135.98(\mathrm{~m}) ;$ HRMS-ESI $(\mathrm{m} / \mathrm{z}):[\mathrm{M}+\mathrm{H}]^{+}$calcd. for $\left[\mathrm{C}_{12} \mathrm{H}_{18} \mathrm{~N}_{2} \mathrm{~F}\right]^{+}$, 209.1449; found, 209.1436. 


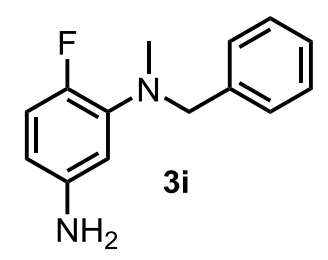

White cream, 20 hours, 50\% yield (using $\mathbf{L 6}$ as ligand); ${ }^{1} \mathrm{H} \mathrm{NMR}\left(500 \mathrm{MHz}, \mathrm{CDCl}_{3}\right.$ ): $\delta$ 7.31-7.23 (m, 5H), 6.84-6.80 (m, 1H), 6.22-6.13 (m, 2H), $4.26(\mathrm{~s}, 2 \mathrm{H}), 3.45$ (brs, 2H), $2.69(\mathrm{~s}, 3 \mathrm{H}) ;{ }^{13} \mathrm{C}$ NMR $\left(125 \mathrm{MHz}, \mathrm{CDCl}_{3}\right): \delta 148.9(\mathrm{~d}, J=233.4 \mathrm{~Hz}), 142.7(\mathrm{~d}, J$ $=1.8 \mathrm{~Hz}), 140.5(\mathrm{~d}, J=1.8 \mathrm{~Hz}), 138.5,128.3,128.2,127.0,116.4(\mathrm{~d}, J=22.1 \mathrm{~Hz})$, $106.8(\mathrm{~d}, J=7.0 \mathrm{~Hz}), 106.2(\mathrm{~d}, J=3.0 \mathrm{~Hz}), 59.22,59.17,38.72,38.70 ;{ }^{19} \mathrm{~F}$ NMR $(470$ $\left.\mathrm{MHz}, \mathrm{CDCl}_{3}\right)$ : -135.52 - $-135.55(\mathrm{~m}) ;$ HRMS-ESI $(\mathrm{m} / \mathrm{z}):[\mathrm{M}+\mathrm{H}]^{+}$calcd. for $\left[\mathrm{C}_{14} \mathrm{H}_{16} \mathrm{~N}_{2} \mathrm{~F}\right]^{+}, 231.1292$; found, 231.1292 .<smiles>CCCCCN(C)c1cc(N)ccc1F</smiles>

White cream, 5 mol\% [Ru(cymene) $\left.\mathrm{Cl}_{2}\right]_{2}, 20$ hours (using $\mathbf{L 2 2}$ as ligand), $43 \%$ yield; ${ }^{1} \mathrm{H}$ NMR $\left(500 \mathrm{MHz}, \mathrm{CDCl}_{3}\right): \delta 6.79-6.75(\mathrm{~m}, 1 \mathrm{H}), 6.22-6.20(\mathrm{~m}, 1 \mathrm{H}), 6.13-6.11(\mathrm{~m}$, $1 \mathrm{H}), 3.47$ (brs, 2H), $3.08(\mathrm{t}, J=7.5 \mathrm{~Hz}, 2 \mathrm{H}), 2.78(\mathrm{~s}, 3 \mathrm{H}), 1.57-1.51(\mathrm{~m}, 2 \mathrm{H})$, $1.34-1.24(\mathrm{~m}, 4 \mathrm{H}), 0.88(\mathrm{t}, J=7.0 \mathrm{~Hz}, 3 \mathrm{H}),{ }^{13} \mathrm{C} \mathrm{NMR}\left(125 \mathrm{MHz}, \mathrm{CDCl}_{3}\right): \delta 148.9(\mathrm{~d}$, $J=232.3 \mathrm{~Hz}), 142.6(\mathrm{~d}, J=2.0 \mathrm{~Hz}), 140.5(\mathrm{~d}, J=9.3 \mathrm{~Hz}), 116.3(\mathrm{~d}, J=22.5 \mathrm{~Hz})$, $106.5(\mathrm{~d}, J=7.5 \mathrm{~Hz}), 106.0$ (d, $J=2.8 \mathrm{~Hz}), 55.18,55.14,39.53,39.52,29.2,26.7$, 22.5, 14.0; ${ }^{19} \mathrm{~F}$ NMR (470 MHz, $\left.\mathrm{CDCl}_{3}\right)$ : -135.52 - -135.57 (m); HRMS-ESI (m/z): $[\mathrm{M}+\mathrm{H}]^{+}$calcd. for $\left[\mathrm{C}_{12} \mathrm{H}_{20} \mathrm{~N}_{2} \mathrm{~F}\right]^{+}, 211.1605$; found, 211.1596.<smiles>Nc1ccc(F)c(NC2CCCCC2)c1</smiles>

White cream, $5 \mathrm{~mol} \%$ [Ru(cymene) $\left.\mathrm{Cl}_{2}\right]_{2}, 20$ hours, 47\% yield (using $\mathbf{L 2 2}$ as ligand); ${ }^{1} \mathrm{H}$ NMR $\left(500 \mathrm{MHz}, \mathrm{CDCl}_{3}\right): \delta 6.75-6.71(\mathrm{~m}, 1 \mathrm{H}), 6.06-6.04(\mathrm{~m}, 1 \mathrm{H}), 5.89-5.86(\mathrm{~m}$, $1 \mathrm{H}), 3.51$ (brs, 3H), 3.21-3.16 (m, 1H), 2.06-2.03 (m, 2H), 1.79-1.75 (m, 2H), $1.67-1.63(\mathrm{~m}, 1 \mathrm{H}), 1.40-1.32(\mathrm{~m}, 2 \mathrm{H}), 1.27-1.14(\mathrm{~m}, 3 \mathrm{H}) ;{ }^{13} \mathrm{C}$ NMR $(125 \mathrm{MHz}$, $\left.\mathrm{CDCl}_{3}\right): \delta 145.8(\mathrm{~d}, J=228.4 \mathrm{~Hz}), 143.0,136.3(\mathrm{~d}, J=11.5 \mathrm{~Hz}), 114.6(\mathrm{~d}, J=19.4$ $\mathrm{Hz}), 102.0(\mathrm{~d}, J=8.0 \mathrm{~Hz}), 99.9(\mathrm{~d}, J=2.1 \mathrm{~Hz}), 51.4,33.3,25.8,25.0 ;{ }^{19} \mathrm{~F}$ NMR $(470$ $\left.\mathrm{MHz}, \mathrm{CDCl}_{3}\right)$ : -148.99 - $-149.04(\mathrm{~m}) ;$ HRMS-ESI $(\mathrm{m} / \mathrm{z}):[\mathrm{M}+\mathrm{H}]^{+}$calcd. for $\left[\mathrm{C}_{12} \mathrm{H}_{18} \mathrm{~N}_{2} \mathrm{~F}\right]^{+}, 209.1449$; found, 209.1439.

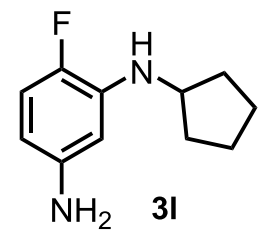

White cream, 5 mol\% $\left[\mathrm{Ru}\left(\text { cymene) } \mathrm{Cl}_{2}\right]_{2}, 20\right.$ hours, 35\% yield (using $\mathbf{L 2 2}$ as ligand); 
${ }^{1} \mathrm{H}$ NMR $\left(500 \mathrm{MHz}, \mathrm{CDCl}_{3}\right): \delta 6.75-6.71(\mathrm{~m}, 1 \mathrm{H}), 6.07-6.05(\mathrm{~m}, 1 \mathrm{H}), 5.90-5.87(\mathrm{~m}$, $1 \mathrm{H}), 3.75-3.70(\mathrm{~m}, 1 \mathrm{H}), 3.44$ (brs, 3H), 2.04-1.97 (m, 2H), 1.77-1.70 (m, 2H), $1.66-1.58(\mathrm{~m}, 2 \mathrm{H}), 1.53-1.46(\mathrm{~m}, 2 \mathrm{H}) ;{ }^{13} \mathrm{C} \mathrm{NMR}\left(125 \mathrm{MHz}, \mathrm{CDCl}_{3}\right): \delta 145.8(\mathrm{~d}, J=$ $227.4 \mathrm{~Hz}), 143.0$ (d, $J=2.1 \mathrm{~Hz}), 136.9$ (d, $J=12.8 \mathrm{~Hz}), 114.4$ (d, $J=19.6 \mathrm{~Hz}), 101.9$ $(\mathrm{d}, J=7.5 \mathrm{~Hz}), 100.1(\mathrm{~d}, J=2.4 \mathrm{~Hz}), 54.3,33.5,33.4,24.0 ;{ }^{19} \mathrm{~F}$ NMR $(470 \mathrm{MHz}$, $\left.\mathrm{CDCl}_{3}\right)$ : -149.01 - -149.06; HRMS-ESI $(\mathrm{m} / \mathrm{z})$ : $[\mathrm{M}+\mathrm{H}]^{+}$calcd. for $\left[\mathrm{C}_{11} \mathrm{H}_{16} \mathrm{~N}_{2} \mathrm{~F}\right]^{+}$, 195.1292; found, 195.1306.

\section{Scheme S1. Comparison between Ru and Ni catalyst}

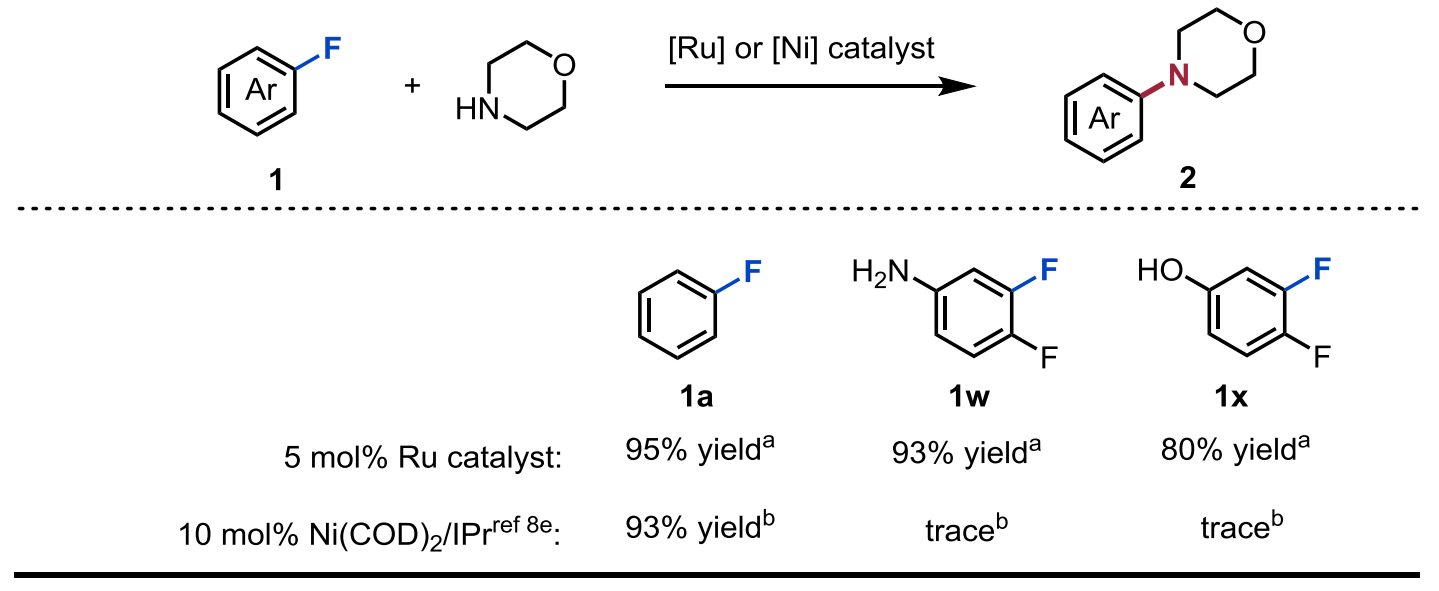<smiles>Fc1ccccc1N1CCOCC1</smiles>

$2 e^{\prime}$

$90 \%$ yield $^{b}$

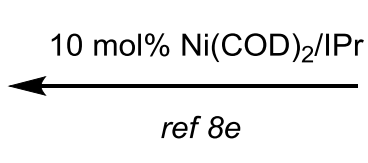

ref $8 e$

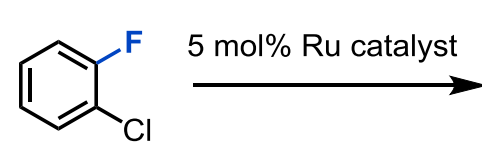

$1 e$<smiles>Clc1ccccc1N1CCOCC1</smiles>

$2 e$

$62 \%$ yield $^{a}$

${ }^{\mathrm{a} C}$ Conditions: 1 (0.40 mmol), $\left[\mathrm{Ru}(\text { cymene }) \mathrm{Cl}_{2}\right]_{2}$ (0.010 mmol), $\operatorname{AgPF}_{6}(0.042 \mathrm{mmol}), \mathbf{L 2 2}(0.040 \mathrm{mmol})$, morpholine $(1.2 \mathrm{mmol}), 4 \AA \mathrm{MS}(20 \mathrm{mg})$, THF $(0.10 \mathrm{~mL}), 120{ }^{\circ} \mathrm{C}, 20$ hours; ${ }^{\mathrm{b} C o n d i t i o n s: ~} 1$ (0.50 mmol), $\mathrm{Ni}(\mathrm{COD})_{2}(0.05 \mathrm{mmol}), \mathrm{IPr} \cdot \mathrm{HCl}(0.1 \mathrm{mmol}),{ }^{t} \mathrm{BuONa}(2.1 \mathrm{mmol})$, morpholine $(2.0 \mathrm{mmol})$, toluene $(3 \mathrm{~mL}), 100{ }^{\circ} \mathrm{C}$, 20 hours. Data are reported as isolated yield of purified compound. 


\section{Mechanism Study}

\subsection{Preparation of Ru-arene $\eta^{6}$-complex}
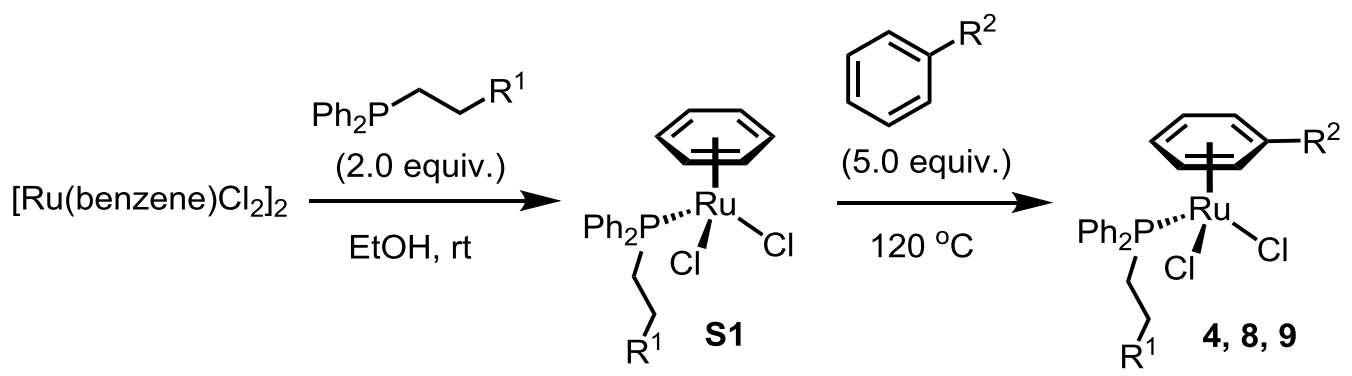

$[\mathrm{Ru}$ (benzene $\left.) \mathrm{Cl}_{2}\right]_{2}(0.1 \mathrm{mmol})$ and phosphine ligand $(0.2 \mathrm{mmol})$ were stirred in EtOH $(0.5 \mathrm{~mL})$ at room temperature overnight. The formed precipitate was filtered and washed by $\mathrm{Et}_{2} \mathrm{O}(5 \mathrm{~mL} \times 2)$, then dried under vaccum. The obtained brown solid was used in next step without further purification.

Complex S1 $(0.1 \mathrm{mmol})$ and arene $(0.5 \mathrm{mmol})$ were mixed together and stirred at $120{ }^{\circ} \mathrm{C}$ under $\mathrm{N}_{2}$ atmosphere for 15 minutes to 1 hour. The mixture was purified by preparative TLC chromatography $(\mathrm{DCM} / \mathrm{EtOH}=30 / 1)$, affording expected product.

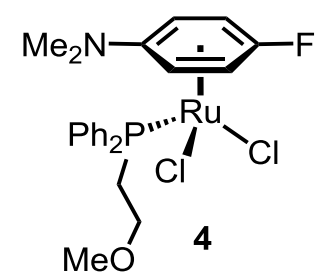

Brown oil, 45\% yield, ${ }^{1} \mathrm{H}$ NMR (500 MHz, $\left.\mathrm{CDCl}_{3}\right): \delta 7.86-7.82(\mathrm{~m}, 4 \mathrm{H}), 7.47-7.43$ (m, 6H), 5.56-5.55 (m, 2H), $4.14(\mathrm{~d}, J=6.0 \mathrm{~Hz}, 2 \mathrm{H}), 3.36-3.31(\mathrm{~m}, 2 \mathrm{H}), 3.09(\mathrm{~s}, 3 \mathrm{H})$, $3.03(\mathrm{~s}, 6 \mathrm{H}), 2.96-2.91(\mathrm{~m}, 2 \mathrm{H}) ;{ }^{13} \mathrm{C} \mathrm{NMR}\left(125 \mathrm{MHz}, \mathrm{CDCl}_{3}\right): \delta 135.3,133.1(\mathrm{~d}, J=$ $9.3 \mathrm{~Hz}), 132.7$ (d, $J=43.4 \mathrm{~Hz}), 130.4(\mathrm{~d}, J=3.5 \mathrm{~Hz}), 128.2$ (d, $J=8.8 \mathrm{~Hz}), 124.5$ (d, $J=275.5 \mathrm{~Hz}), 82.2(\mathrm{~d}, J=23.6 \mathrm{~Hz}), 67.8,58.0(\mathrm{~d}, J=3.1 \mathrm{~Hz}), 57.9(\mathrm{~d}, J=3.6 \mathrm{~Hz})$, 40.9, $26.0(\mathrm{~d}, J=27.0 \mathrm{~Hz}) ;{ }^{31} \mathrm{P}$ NMR $\left(202 \mathrm{MHz}, \mathrm{CDCl}_{3}\right): 23.3 ;{ }^{19} \mathrm{~F}$ NMR $(470 \mathrm{MHz}$, $\left.\mathrm{CDCl}_{3}\right)$ : -170.2; HRMS-ESI (m/z): $[\mathrm{M}-\mathrm{Cl}]^{+}$calcd. for $\left[\mathrm{C}_{23} \mathrm{H}_{27} \mathrm{NOFPClRu}\right]^{+}, 520.0541$; found, 520.0545 .

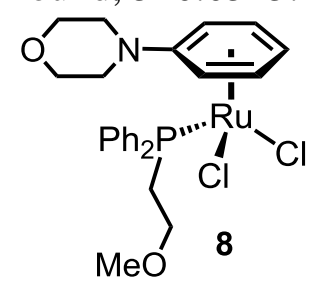

Brown oil, 70\% yield, ${ }^{1} \mathrm{H}$ NMR $\left(500 \mathrm{MHz}, \mathrm{CDCl}_{3}\right): \delta 7.88-7.85(\mathrm{~m}, 4 \mathrm{H}), 7.47-7.44$ $(\mathrm{m}, 6 \mathrm{H}), 5.43-5.41(\mathrm{~m}, 2 \mathrm{H}), 4.57(\mathrm{~d}, J=6.0 \mathrm{~Hz}, 2 \mathrm{H}), 4.13(\mathrm{t}, J=5.0 \mathrm{~Hz}, 1 \mathrm{H}), 3.82(\mathrm{t}$, $J=5.0 \mathrm{~Hz}, 4 \mathrm{H}), 3.55(\mathrm{t}, J=5.5 \mathrm{~Hz}, 4 \mathrm{H}), 3.33-3.28(\mathrm{~m}, 2 \mathrm{H}), 3.07(\mathrm{~s}, 3 \mathrm{H}), 2.90-2.85$ $(\mathrm{m}, 2 \mathrm{H}) ;{ }^{13} \mathrm{C}$ NMR $\left(125 \mathrm{MHz}, \mathrm{CDCl}_{3}\right): \delta 136.4,133.5(\mathrm{~d}, J=43.3 \mathrm{~Hz}), 132.8(\mathrm{~d}, J=$ $9.1 \mathrm{~Hz}), 130.3(\mathrm{~d}, J=1.5 \mathrm{~Hz}), 128.2(\mathrm{~d}, J=9.4 \mathrm{~Hz}), 92.5,71.1,67.7,66.3,61.3(\mathrm{~d}, J=$ $9.0 \mathrm{~Hz}), 57.9,45.9,25.7(\mathrm{~d}, J=27.1 \mathrm{~Hz}) ;{ }^{31} \mathrm{P} \mathrm{NMR}\left(202 \mathrm{MHz}, \mathrm{CDCl}_{3}\right): 24.1$; HRMS-ESI $(\mathrm{m} / \mathrm{z}):[\mathrm{M}-\mathrm{Cl}]^{+}$calcd. for $\left[\mathrm{C}_{25} \mathrm{H}_{30} \mathrm{NO}_{2} \mathrm{PClRu}\right]^{+}, 544.0741$; found, 
544.0752.

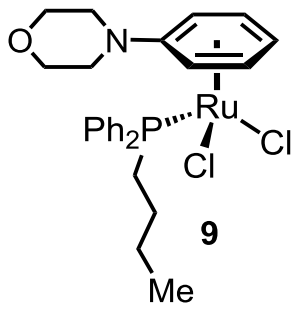

Brown oil, 50\% yield, ${ }^{1} \mathrm{H}$ NMR $\left(600 \mathrm{MHz}, \mathrm{CDCl}_{3}\right): \delta 7.89-7.86(\mathrm{~m}, 4 \mathrm{H}), 7.47-7.46$ $(\mathrm{m}, 6 \mathrm{H}), 5.45-5.43(\mathrm{~m}, 2 \mathrm{H}), 4.52(\mathrm{~d}, J=6.0 \mathrm{~Hz}, 2 \mathrm{H}), 4.11(\mathrm{t}, J=4.8 \mathrm{~Hz}, 1 \mathrm{H}), 3.80(\mathrm{t}$, $J=5.4 \mathrm{~Hz}, 4 \mathrm{H}), 3.51(\mathrm{t}, J=5.4 \mathrm{~Hz}, 4 \mathrm{H}), 2.56-2.52(\mathrm{~m}, 2 \mathrm{H}), 1.21-1.18(\mathrm{~m}, 2 \mathrm{H})$, $1.12-1.08(\mathrm{~m}, 2 \mathrm{H}), 0.72(\mathrm{t}, J=7.8 \mathrm{~Hz}, 3 \mathrm{H}) ;{ }^{13} \mathrm{C} \mathrm{NMR}\left(150 \mathrm{MHz}, \mathrm{CDCl}_{3}\right): \delta 136.0$, $133.3(\mathrm{~d}, J=42.5 \mathrm{~Hz}), 132.9(\mathrm{~d}, J=8.9 \mathrm{~Hz}), 130.1(\mathrm{~d}, J=1.8 \mathrm{~Hz}), 128.1(\mathrm{~d}, J=9.8$ $\mathrm{Hz}), 92.3,71.2,66.3,61.3$ (d, $J=7.7 \mathrm{~Hz}), 45.9,25.4$ (d, $J=8.4 \mathrm{~Hz}), 23.9$ (d, $J=13.2$ $\mathrm{Hz}), 23.4$ (d, $J=27.5 \mathrm{~Hz}), 13.7 ;{ }^{31} \mathrm{P}$ NMR (202 MHz, $\left.\mathrm{CDCl}_{3}\right)$ : 29.0; HRMS-ESI (m/z): $[\mathrm{M}-\mathrm{Cl}]^{+}$calcd. for $\left[\mathrm{C}_{26} \mathrm{H}_{32} \mathrm{NOPClRu}\right]^{+}$, 542.0948; found, 542.0952.

\section{2 $S_{N} A r$ amination of Ru-fluoroarene $\eta^{6}$-complex}
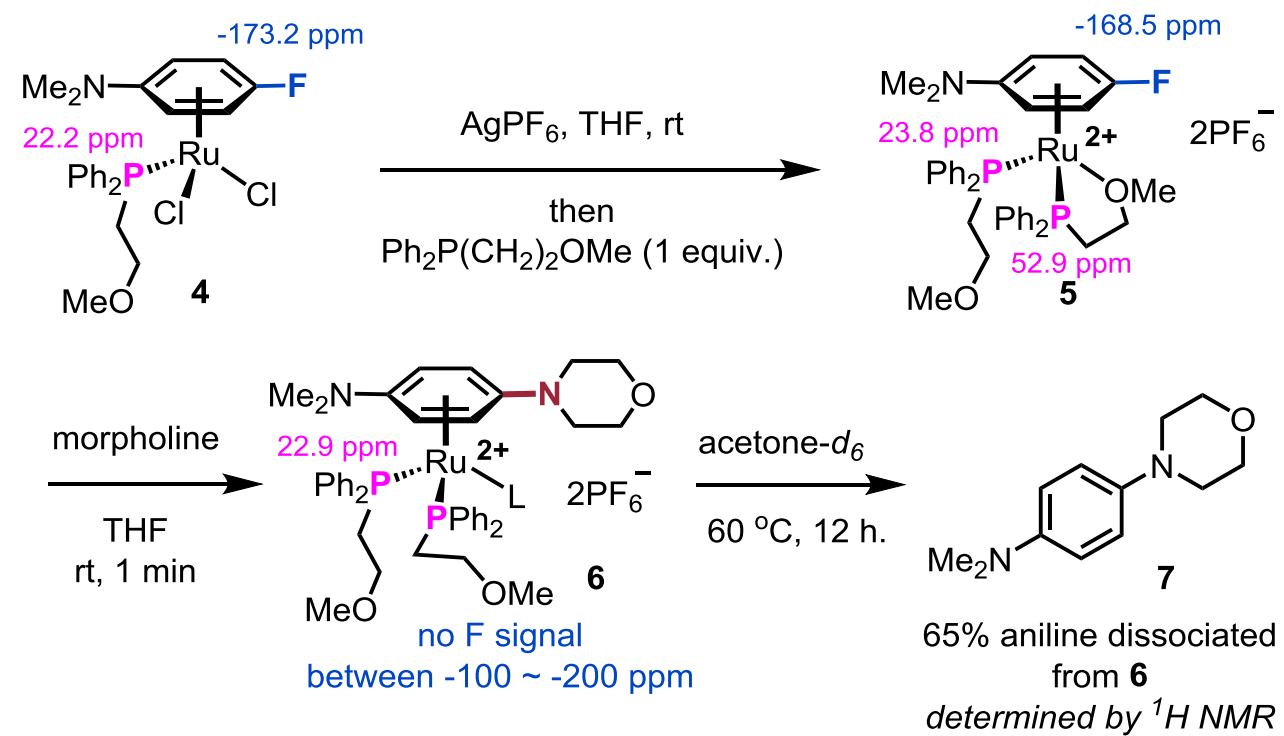

Note: no free fluoroarene was detected by ${ }^{19}$ F NMR during the whole process.

Under an atmosphere of $\mathrm{N}_{2}$, Ru-fluoroarene $\eta^{6}$-complex $4(0.02 \mathrm{mmol})$ and $\mathrm{AgPF}_{6}$ $(0.04 \mathrm{mmol})$ were stirred in THF $(1.0 \mathrm{~mL})$ at room temperature for 15 minutes. Precipitates were filtered off, and then ligand $\mathbf{L 2 2}(0.02 \mathrm{mmol})$ was added to the filtrate. The mixture was stirred at room temperature for another 15 minutes. THF was excluded under reduced pressure, affording complex 5.

To the solution of 5 in acetone- $d_{6}(0.5 \mathrm{~mL})$, morpholine $(1.2 \mathrm{mmol})$ was added and the mixture was monitored by ${ }^{19} \mathrm{~F}$ NMR and ${ }^{31} \mathrm{P}$ NMR, showing a quick disappearance of fluorine peaks within 1 minute. During the above investigations, no signal of free fluoroarene was detected by ${ }^{19} \mathrm{~F}$ NMR.

The reaction mixture was heated at $60{ }^{\circ} \mathrm{C}$ for 12 hours and monitored by ${ }^{1} \mathrm{H}$ NMR. $65 \%$ aniline dissociated from the $\mathrm{Ru}$-complex. 

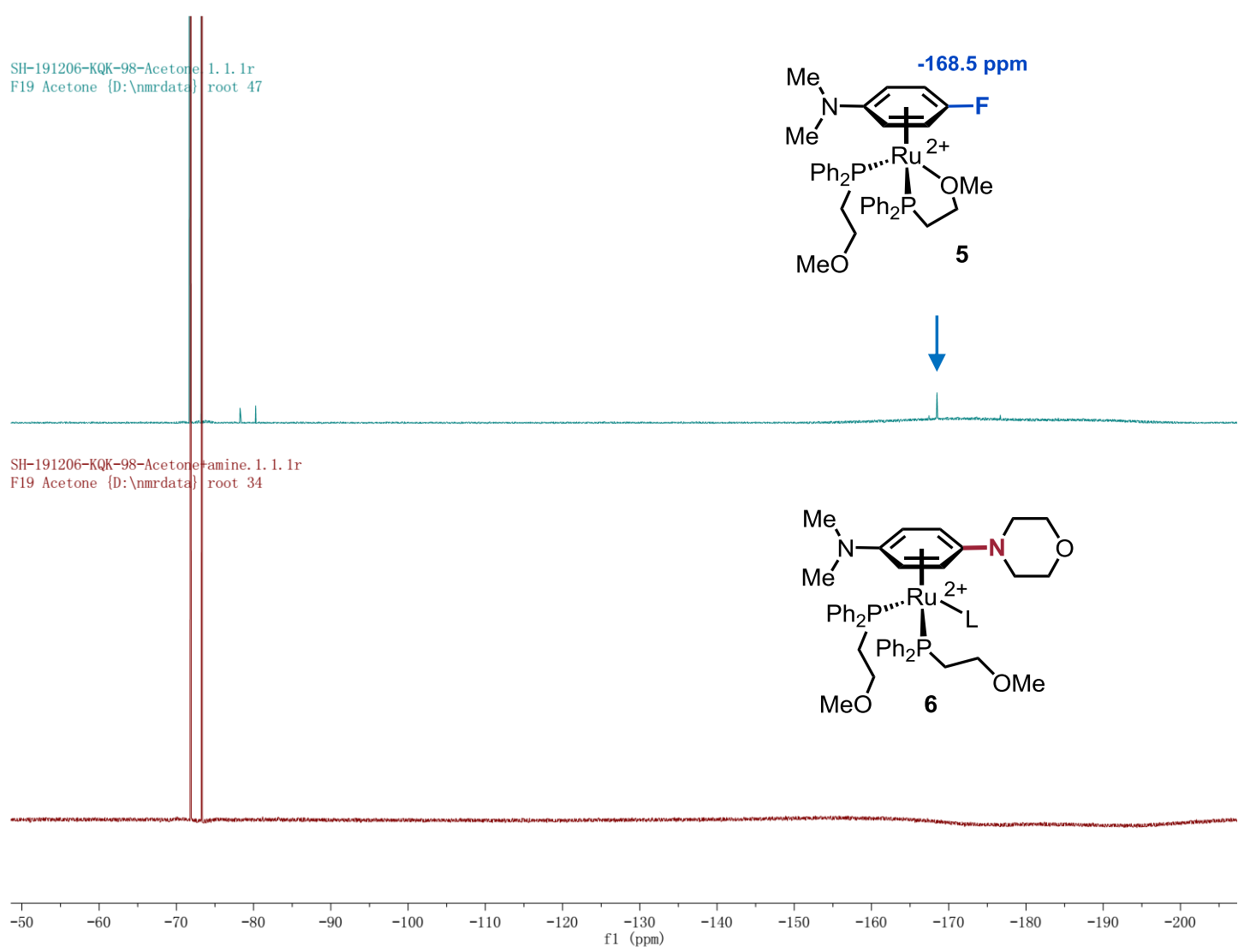

Figure S2. ${ }^{19}$ F NMR of intermediate 5 and 6

SH-191206-KQK-98-Acetone. $3.1 .1 \mathrm{r}$
P31 Acetone \{D: \nmrdata $\}$ root 47
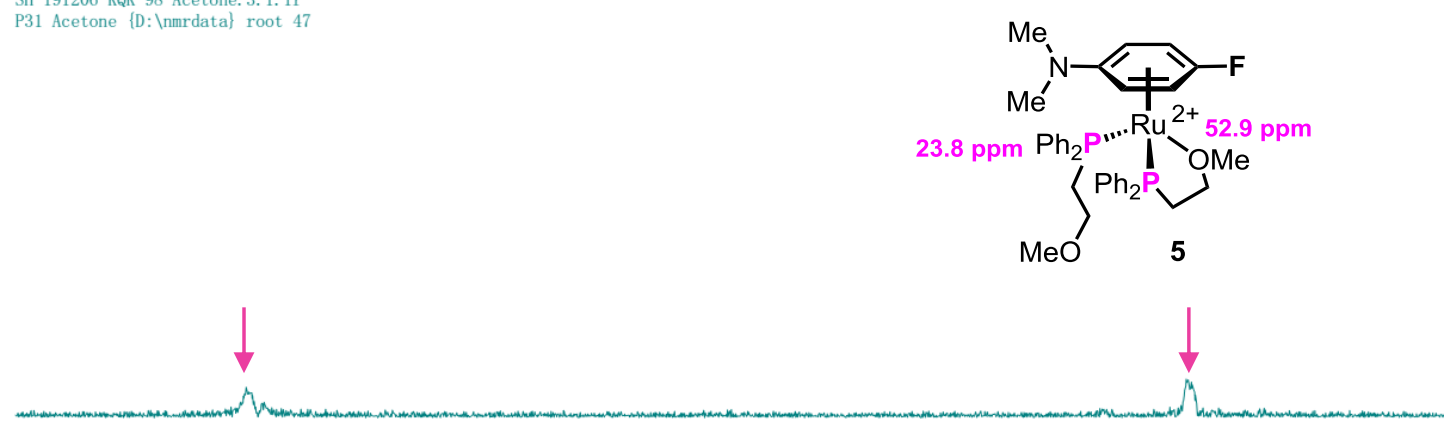

SH-191206-KQK-98-Acetone+amine. 3. 1. 1r

P31 Acetone $\{\mathrm{D}: \backslash$ nmrdata $\}$ root 34
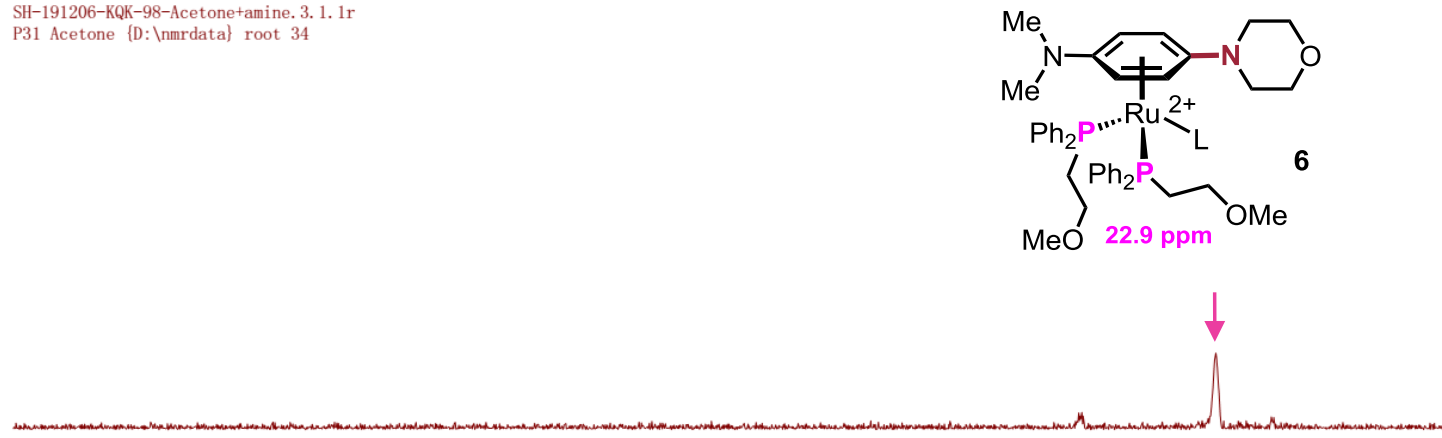

55

45

f1 (ppm)

30

120

Figure S3. ${ }^{31} \mathrm{P}$ NMR of intermediate 5 and 6 


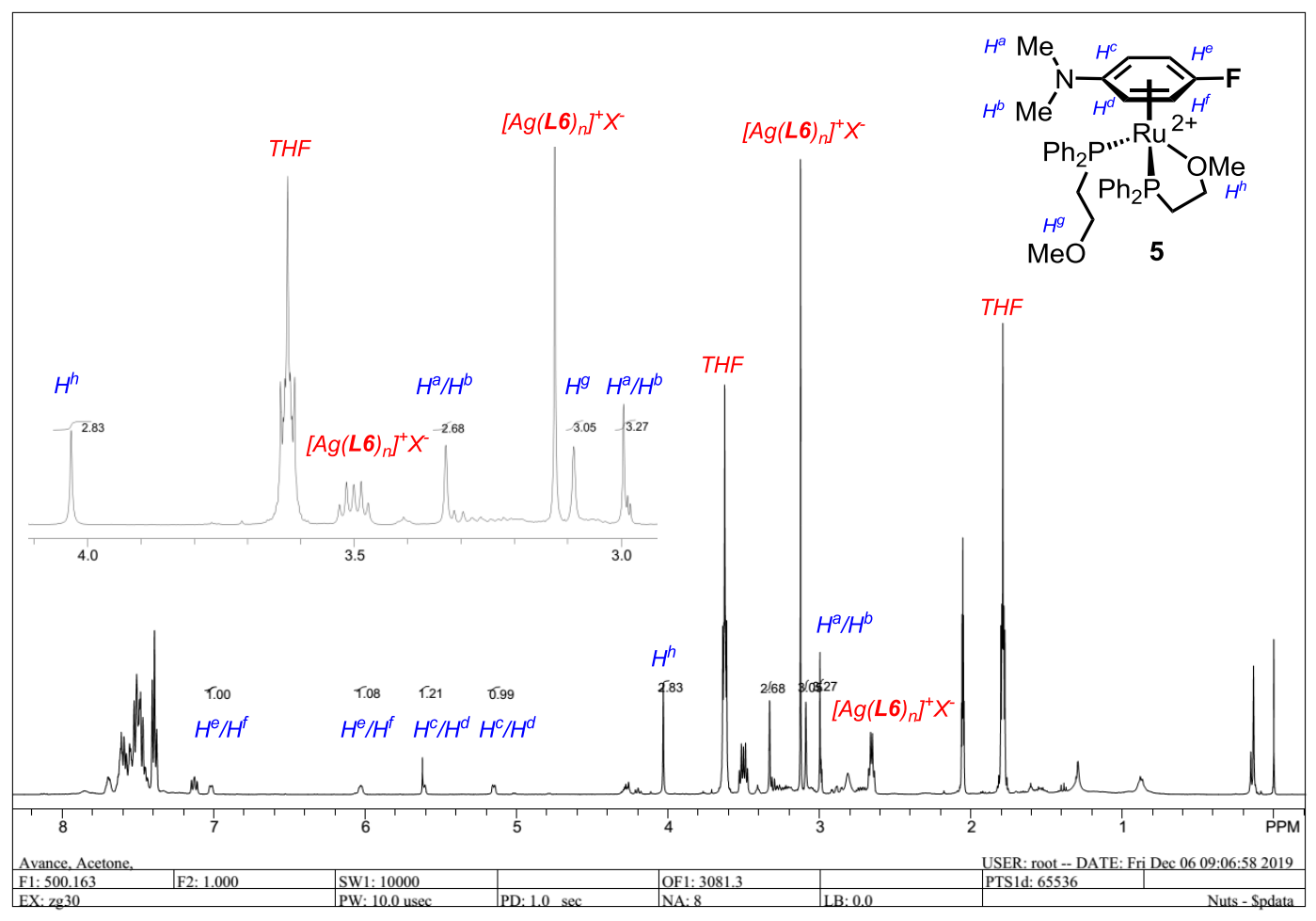

Figure S4. ${ }^{1} \mathrm{H}$ NMR of intermediate 5

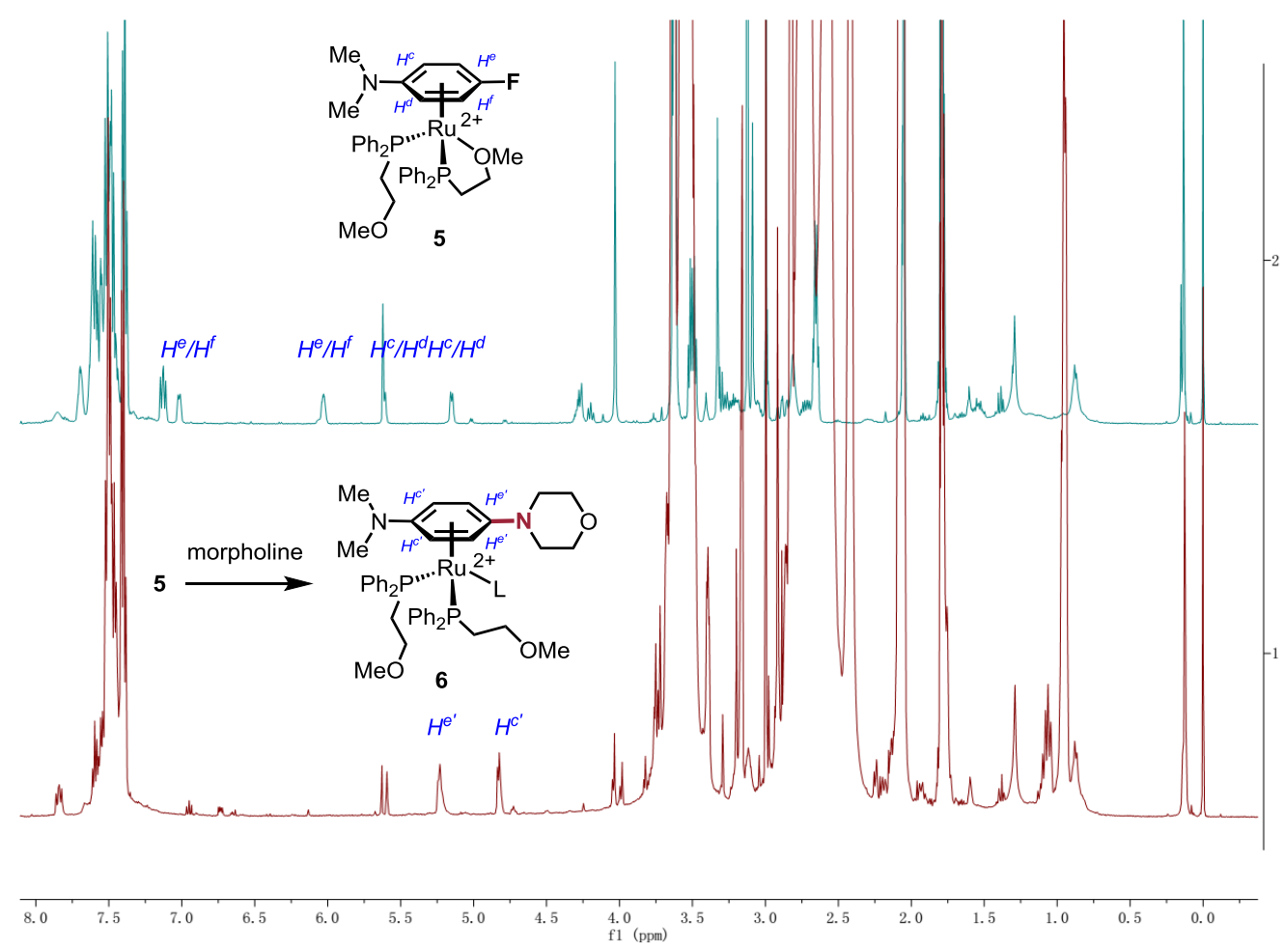

Figure S5. ${ }^{1} \mathrm{H}$ NMR of intermediate 5 and 6 


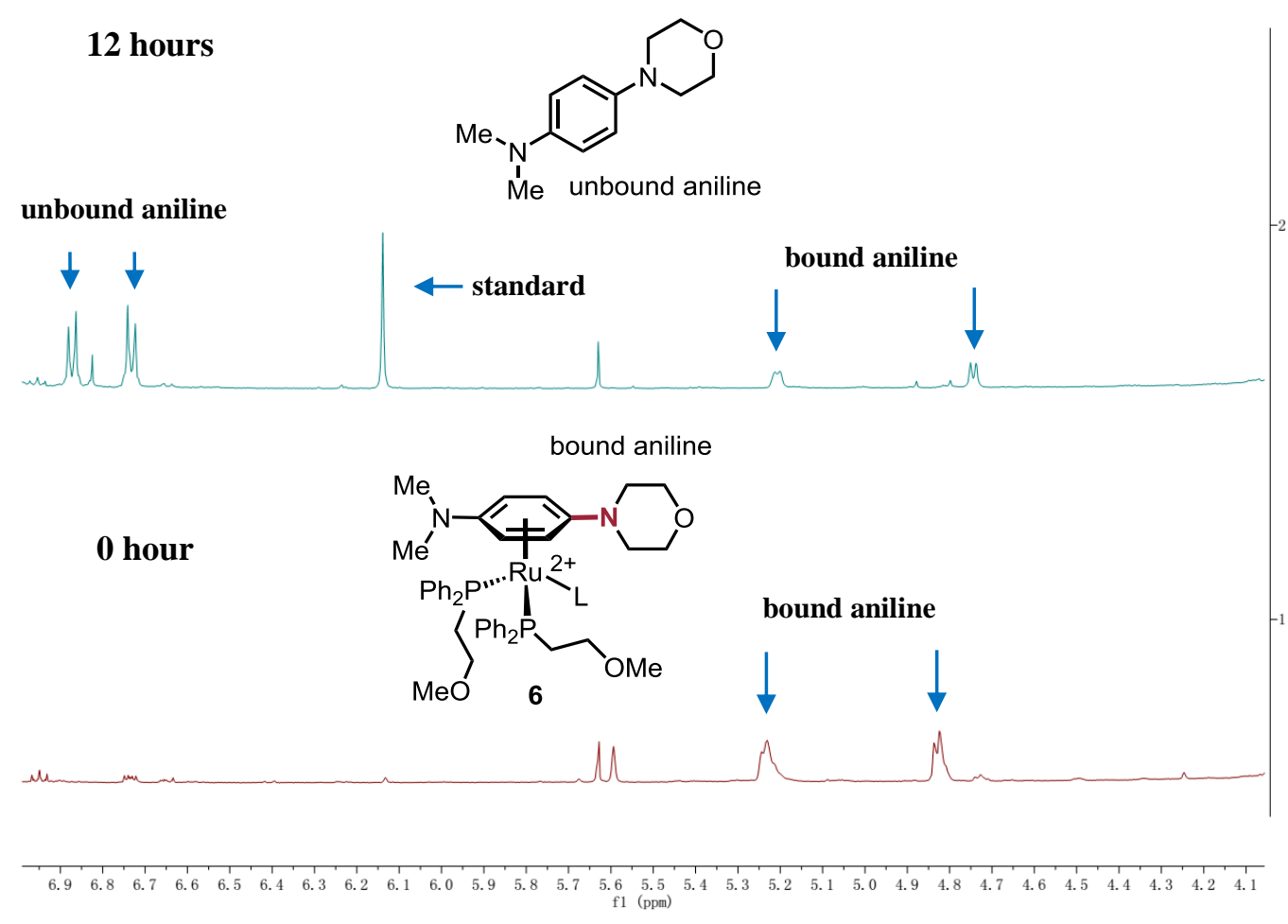

Figure S6. Aniline dissociation over time

\subsection{Arene dissociation test}
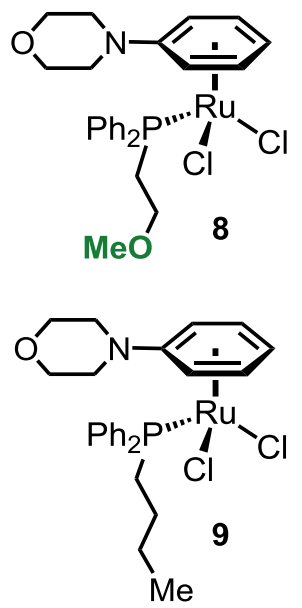
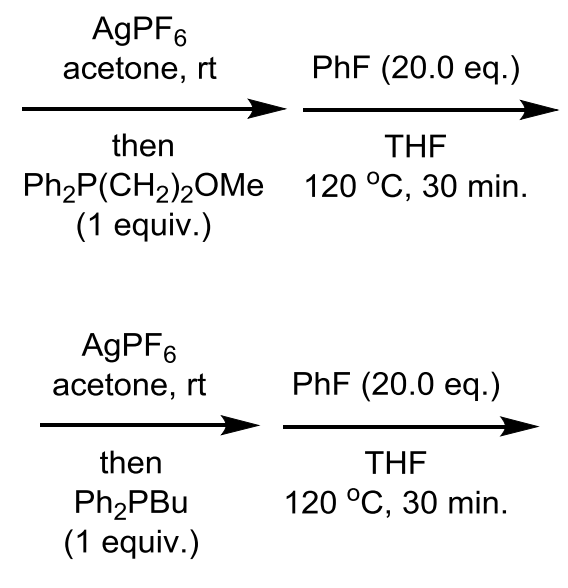<smiles>O=S(=O)(O)OC1COCCN(c2ccccc2)c2ccccc21</smiles><smiles>c1ccc(N2CCOCC2)cc1</smiles>

Under an atmosphere of $\mathrm{N}_{2}$, Ru-aniline $\eta^{6}$-complex 8 or $9(0.020 \mathrm{mmol})$ and $\mathrm{AgPF}_{6}$ $(0.040 \mathrm{mmol})$ were stirred in acetone $(1.0 \mathrm{~mL})$ at room temperature for 15 minutes. Formed $\mathrm{AgCl}$ precipitate was filtered off, then ligand $(0.020 \mathrm{mmol})$ was added to the acetone solution and stirred for another 15 minutes. After acetone was excluded under reduced pressure, PhF $(0.40 \mathrm{mmol})$ and THF $(0.1 \mathrm{~mL})$ were added under $\mathrm{N}_{2}$. The whole mixture was stirred at $120{ }^{\circ} \mathrm{C}$ for 30 minutes. After cooling to room temperature, morpholine $(1.2 \mathrm{mmol})$ was added to neutralize generated acid. The yield of released free aniline was determined by ${ }^{1} \mathrm{H}$ NMR using 1,1,2,2-tetrachloroethane as the internal standard. 


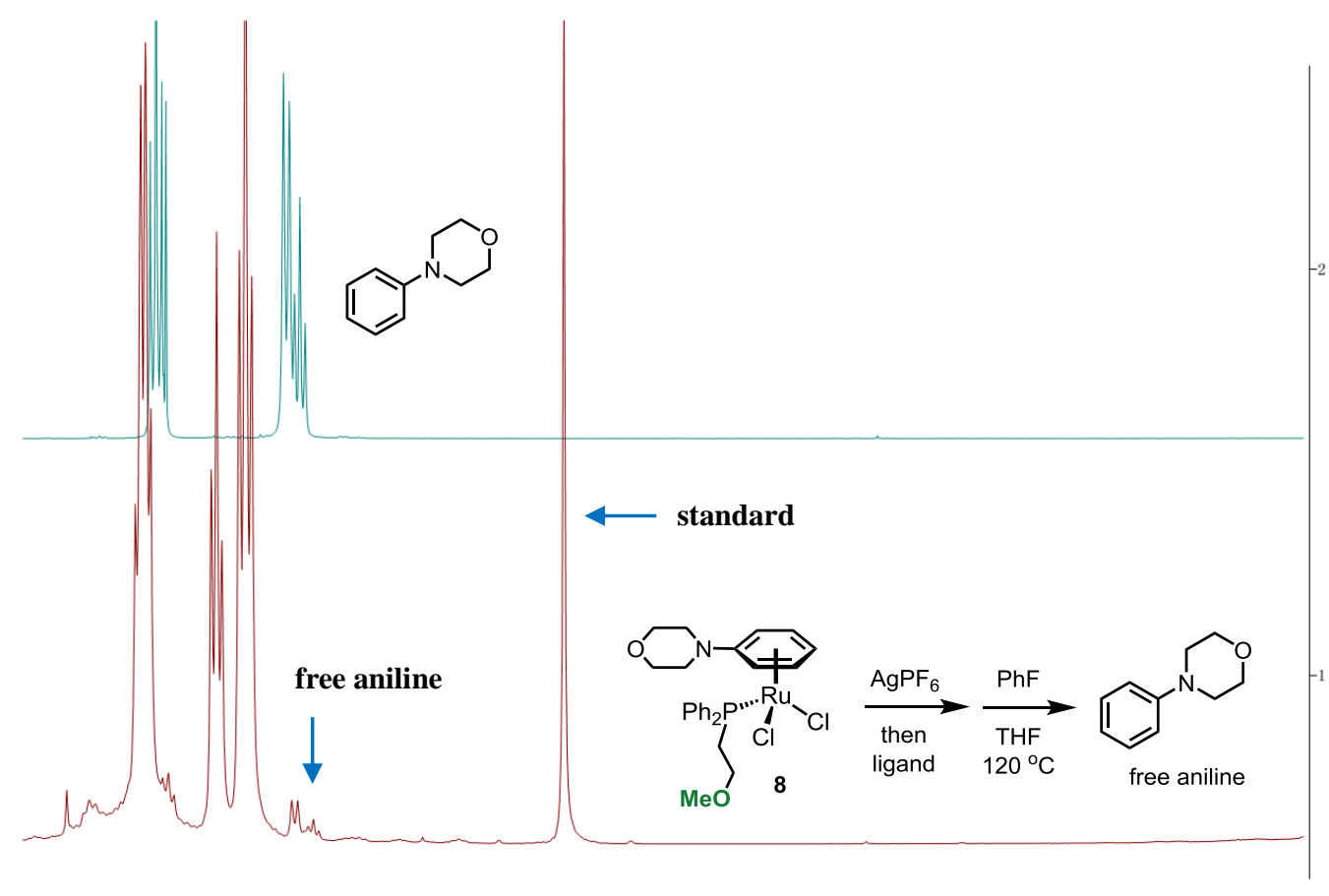

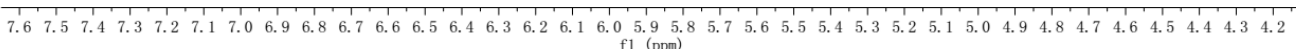
f1 (ppm)

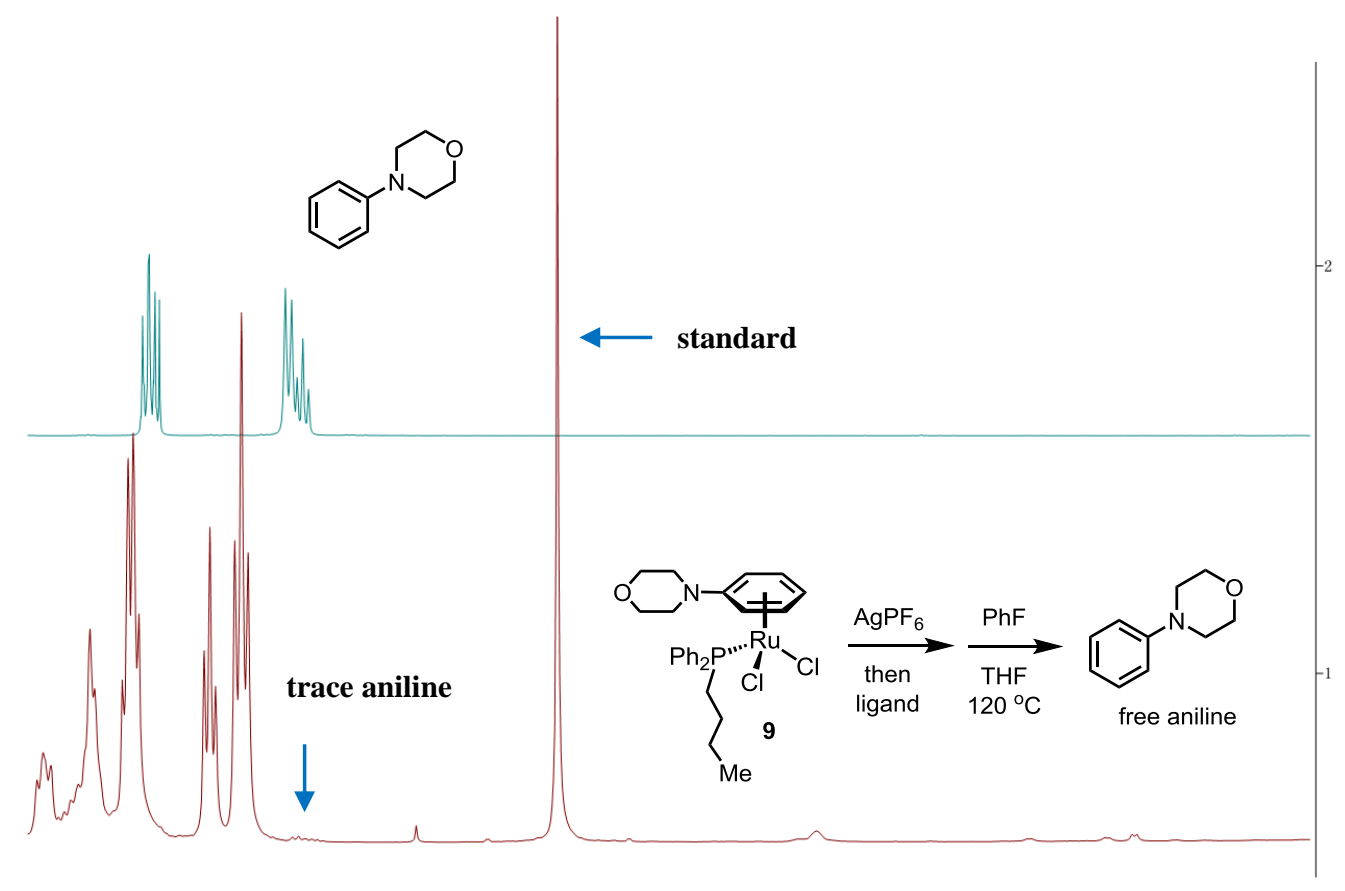

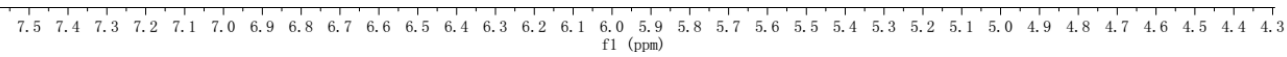

Figure S7. Arene dissociation test 


\subsection{Arene exchange test}

a.

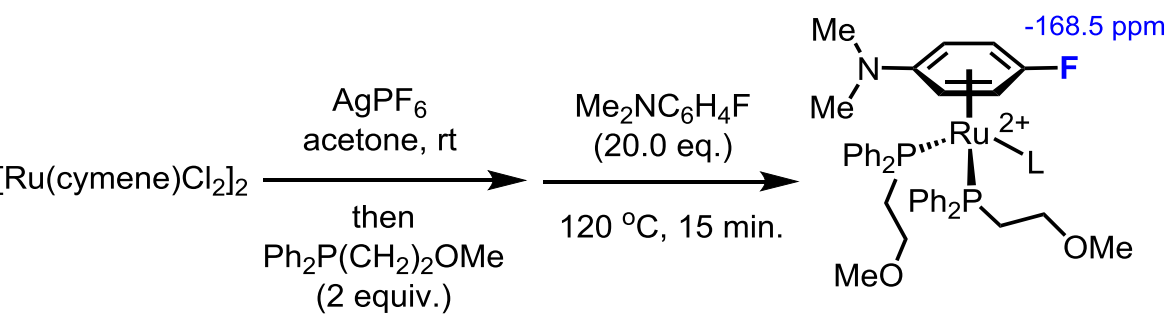

b.

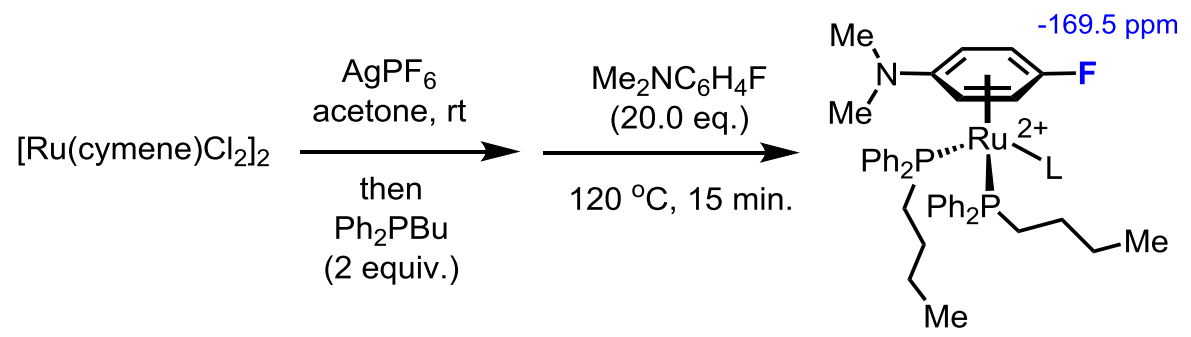

Under $\mathrm{N}_{2}$ atmosphere, $\left[\mathrm{Ru}(\mathrm{cymene}) \mathrm{Cl}_{2}\right]_{2}(0.010 \mathrm{mmol})$ and $\mathrm{AgPF}_{6}(0.040 \mathrm{mmol})$ were stirred in acetone $(1.0 \mathrm{~mL})$ at room temperature for 15 minutes. The formed $\mathrm{AgCl}$ precipitate was filtered off and acetone solution was transferred to a $4 \mathrm{~mL}$ vial which contained ligand $(0.040 \mathrm{mmol})$. Then acetone was excluded under reduced pressure, and $\mathrm{Me}_{2} \mathrm{NC}_{6} \mathrm{H}_{4} \mathrm{~F}(0.4 \mathrm{mmol})$ was added. The mixture was stirred at $120{ }^{\circ} \mathrm{C}$ for 15 minutes, and then cooled to room temperature. Fluorine peak at $-169.5 \mathrm{ppm}$ and $-168.5 \mathrm{ppm}\left({ }^{19} \mathrm{~F}\right.$ NMR in THF) were observed for reaction $\mathbf{4 . 4 a}$ and $\mathbf{4 . 4 b}$ respectively, which support the formation of Ru-fluoroarene $\eta^{6}$-complex.

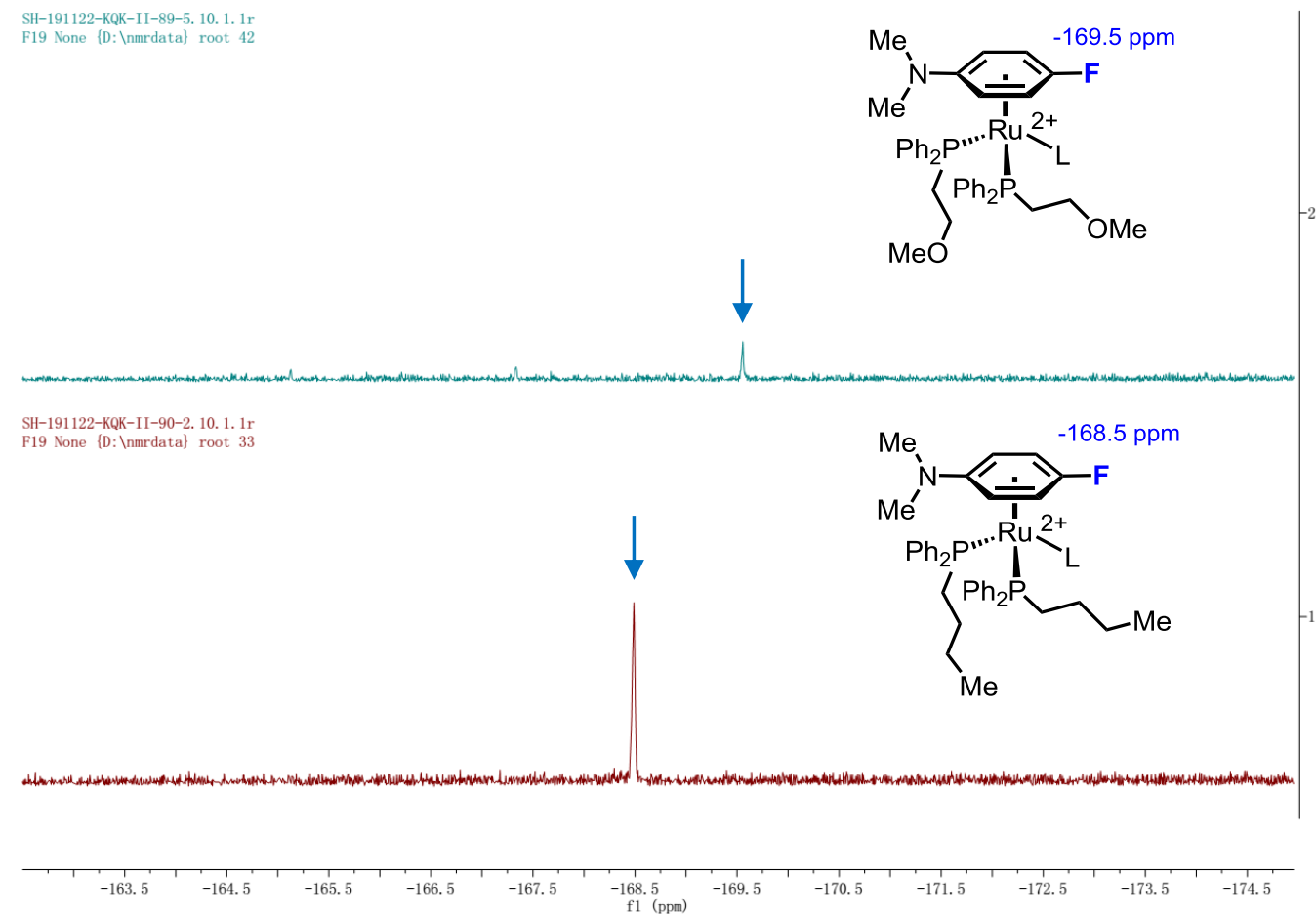

Figure S8. Arene exchange monitored by ${ }^{19}$ F NMR 


\section{Gram-Scale Experiment}
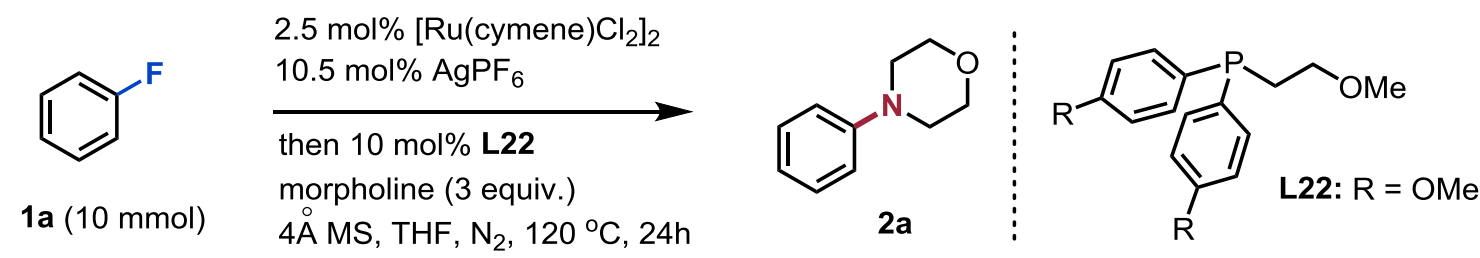

$93 \%$ yield, $1.51 \mathrm{~g}$

Under $\mathrm{N}_{2}$ atmosphere, $\left[\mathrm{Ru}(\mathrm{cymene}) \mathrm{Cl}_{2}\right]_{2}(0.25 \mathrm{mmol})$ and $\mathrm{AgPF}_{6}(1.05 \mathrm{mmol})$ were stirred in acetone $(10 \mathrm{~mL})$ at room temperature for 15 minutes. The formed $\mathrm{AgCl}$ precipitate was filtered off and acetone solution was transferred to a $20 \mathrm{~mL}$ sealed tube which contained ligand $(1.0 \mathrm{mmol})$. Then acetone was excluded under reduced pressure, affording the catalyst as yellow foam. $4 \AA$ MS (1.0 g), aryl fluoride (10 $\mathrm{mmol}$ ), amine $(30 \mathrm{mmol})$ and $2.5 \mathrm{~mL}$ THF were added into the tube. The reaction mixture was stirred at $120{ }^{\circ} \mathrm{C}$ for 24 hours under $\mathrm{N}_{2}$ atmosphere. Then, the mixture was purified by silica gel chromatography (ethyl acetate/ petroleum ether), affording $1.51 \mathrm{~g}$ product, $93 \%$ isolated yield. 


\section{NMR Spectra}

${ }^{1} \mathrm{H}$ NMR $\left(500 \mathrm{MHz}, \mathrm{CDCl}_{3}\right)$ :

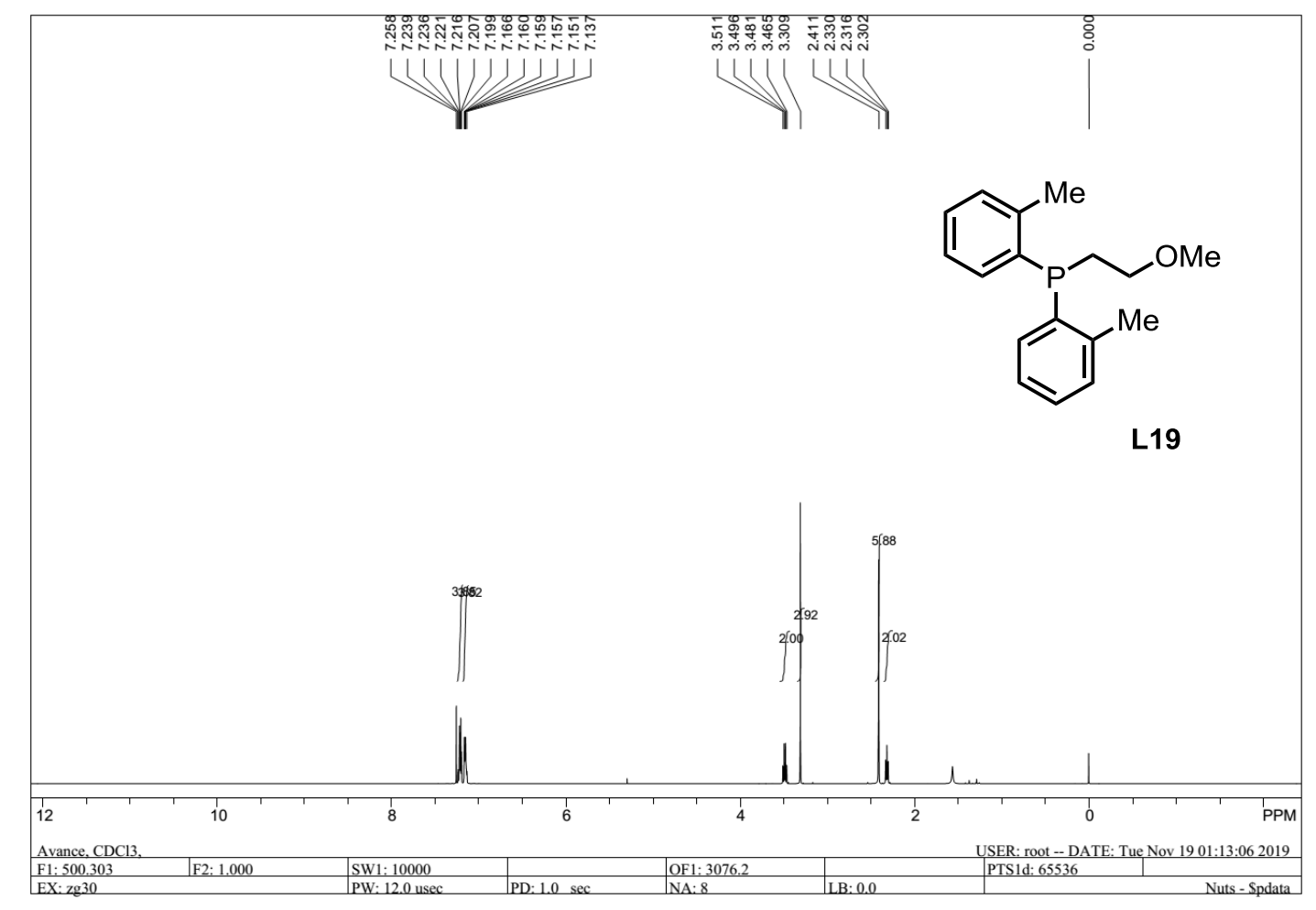

${ }^{13} \mathrm{C}$ NMR $\left(125 \mathrm{MHz}, \mathrm{CDCl}_{3}\right)$ :

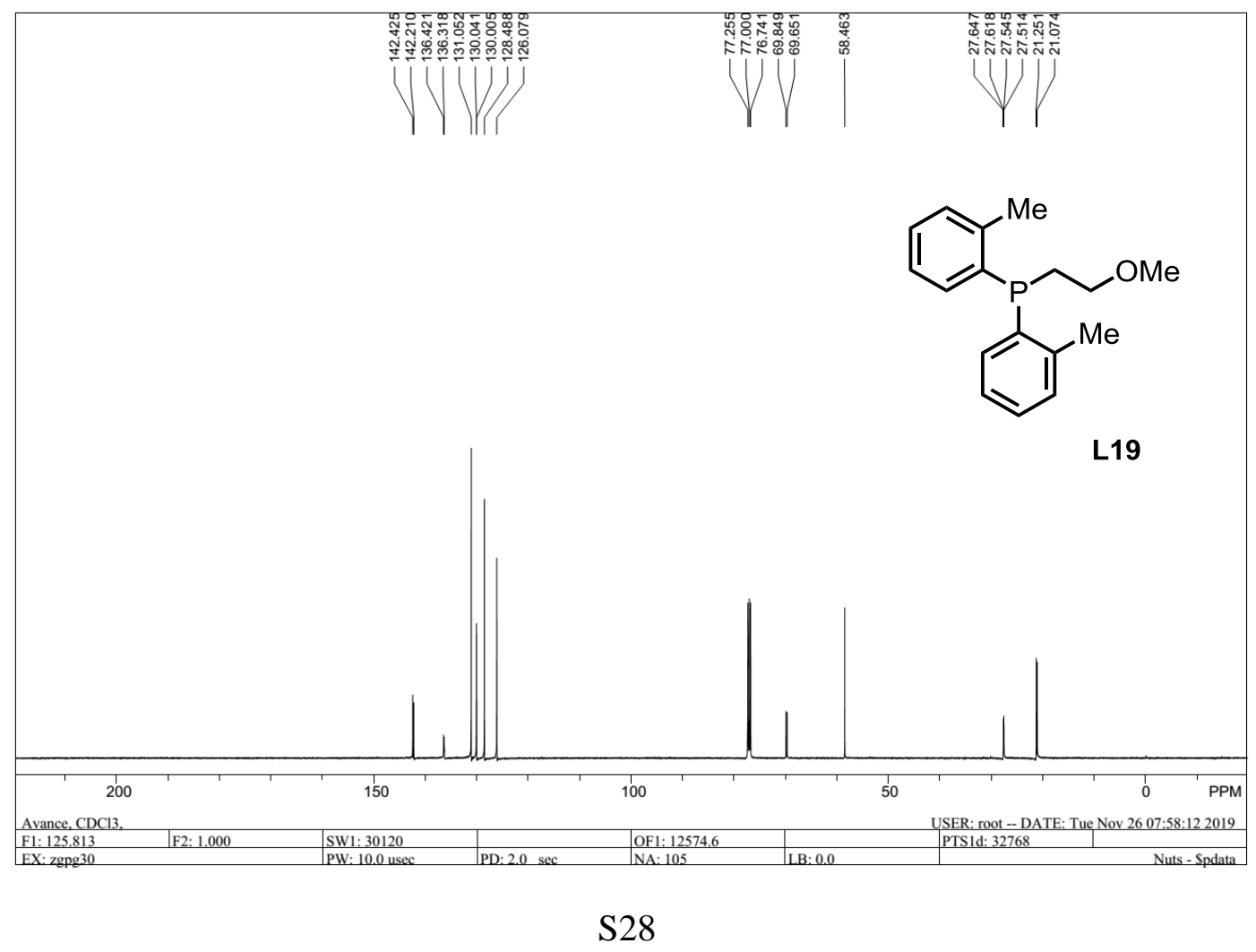


${ }^{31}$ P NMR (202 MHz, $\left.\mathrm{CDCl}_{3}\right)$ :

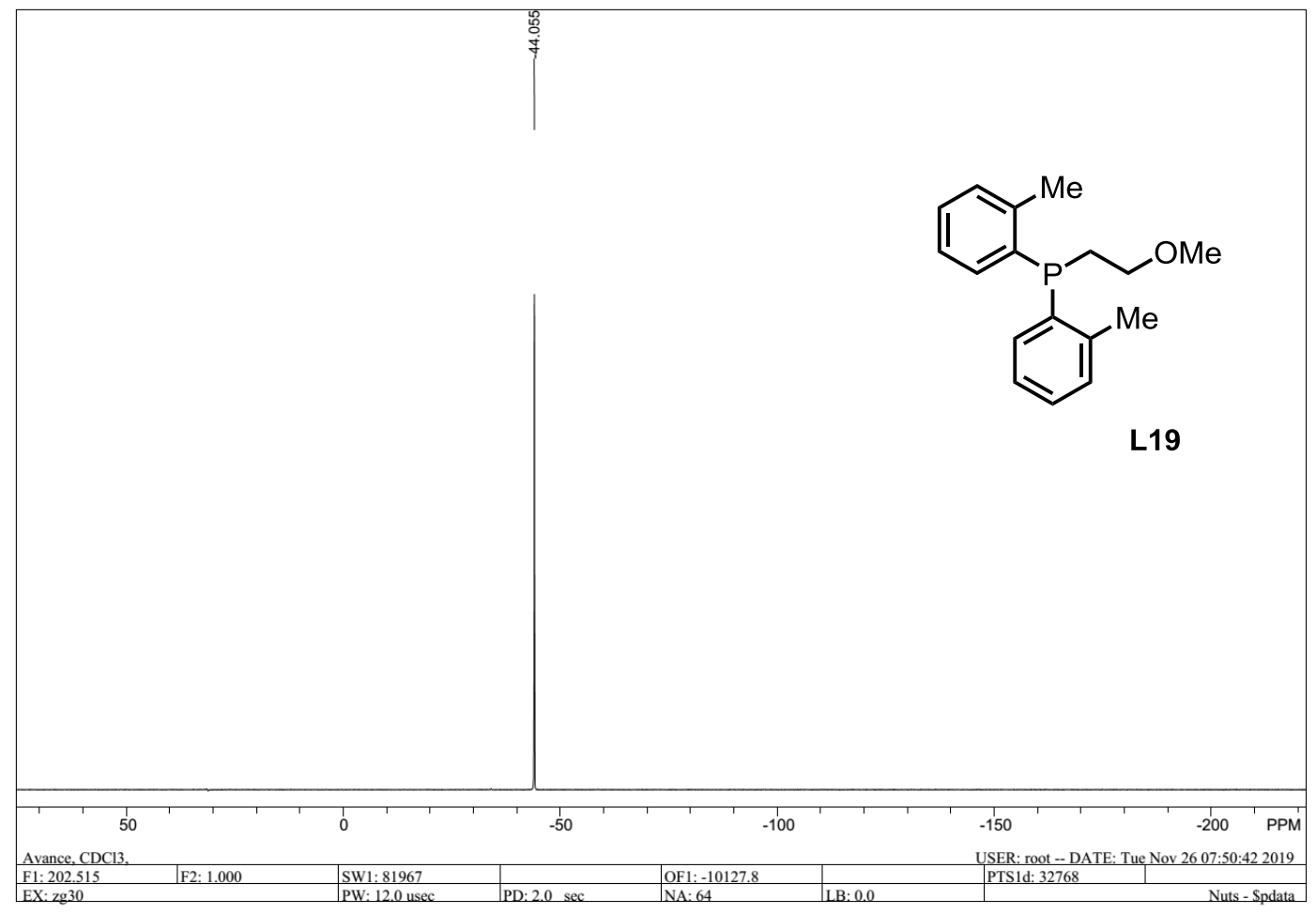


${ }^{1} \mathrm{H}$ NMR $\left(500 \mathrm{MHz}, \mathrm{CDCl}_{3}\right)$ :

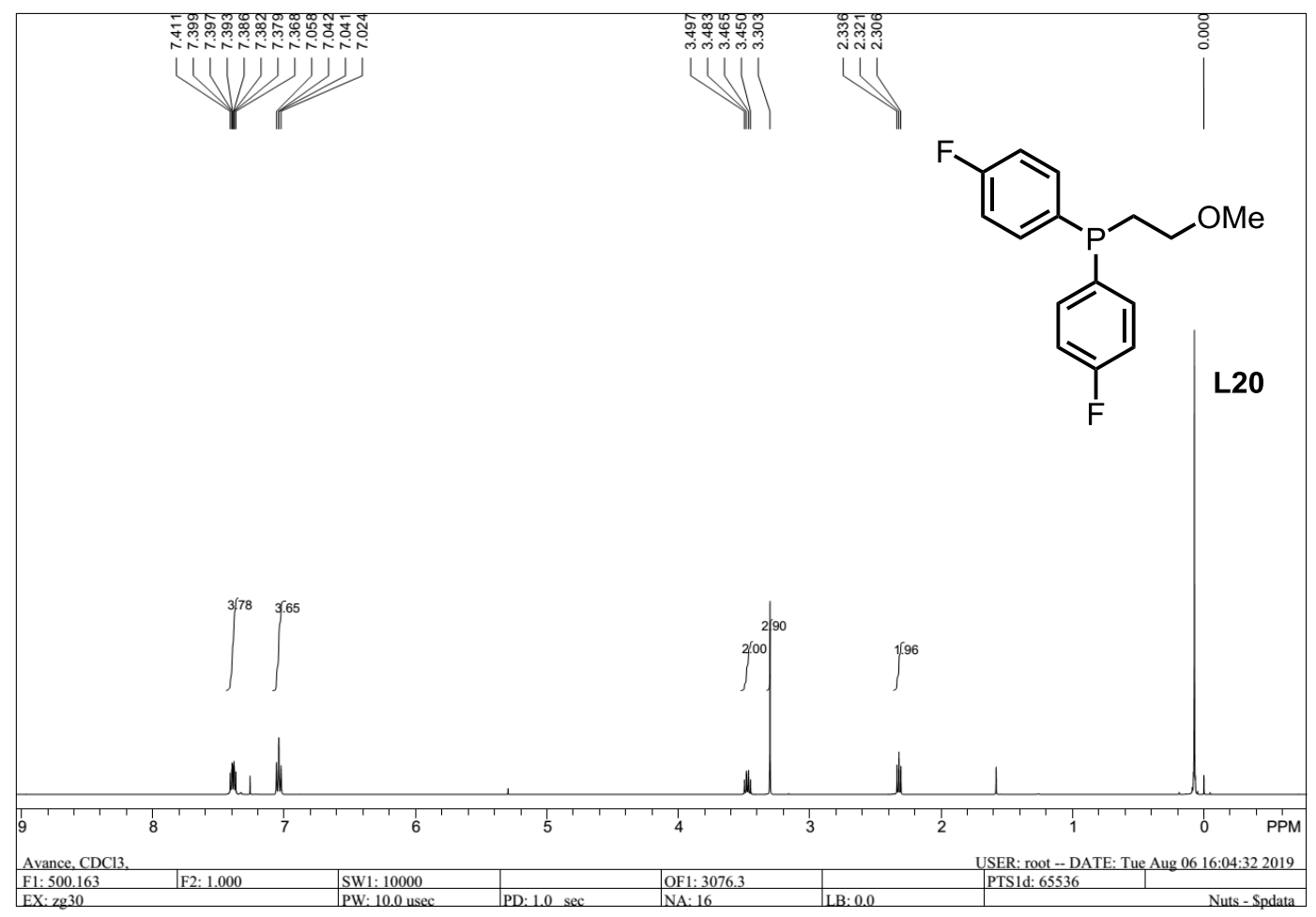

${ }^{13} \mathrm{C}$ NMR $\left(125 \mathrm{MHz}, \mathrm{CDCl}_{3}\right)$ :

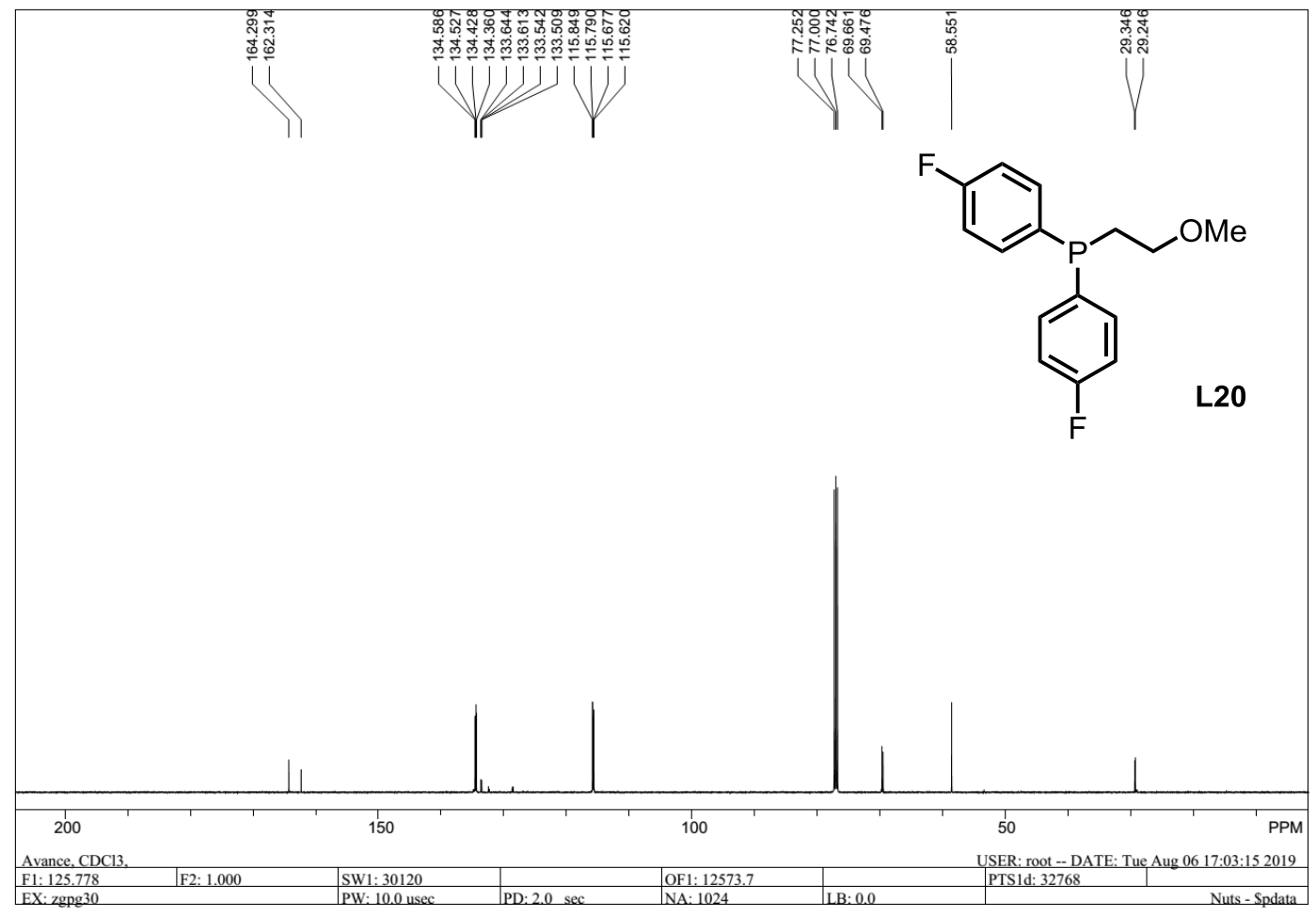


${ }^{19} \mathrm{~F}$ NMR (470 MHz, $\mathrm{CDCl}_{3}$ ):

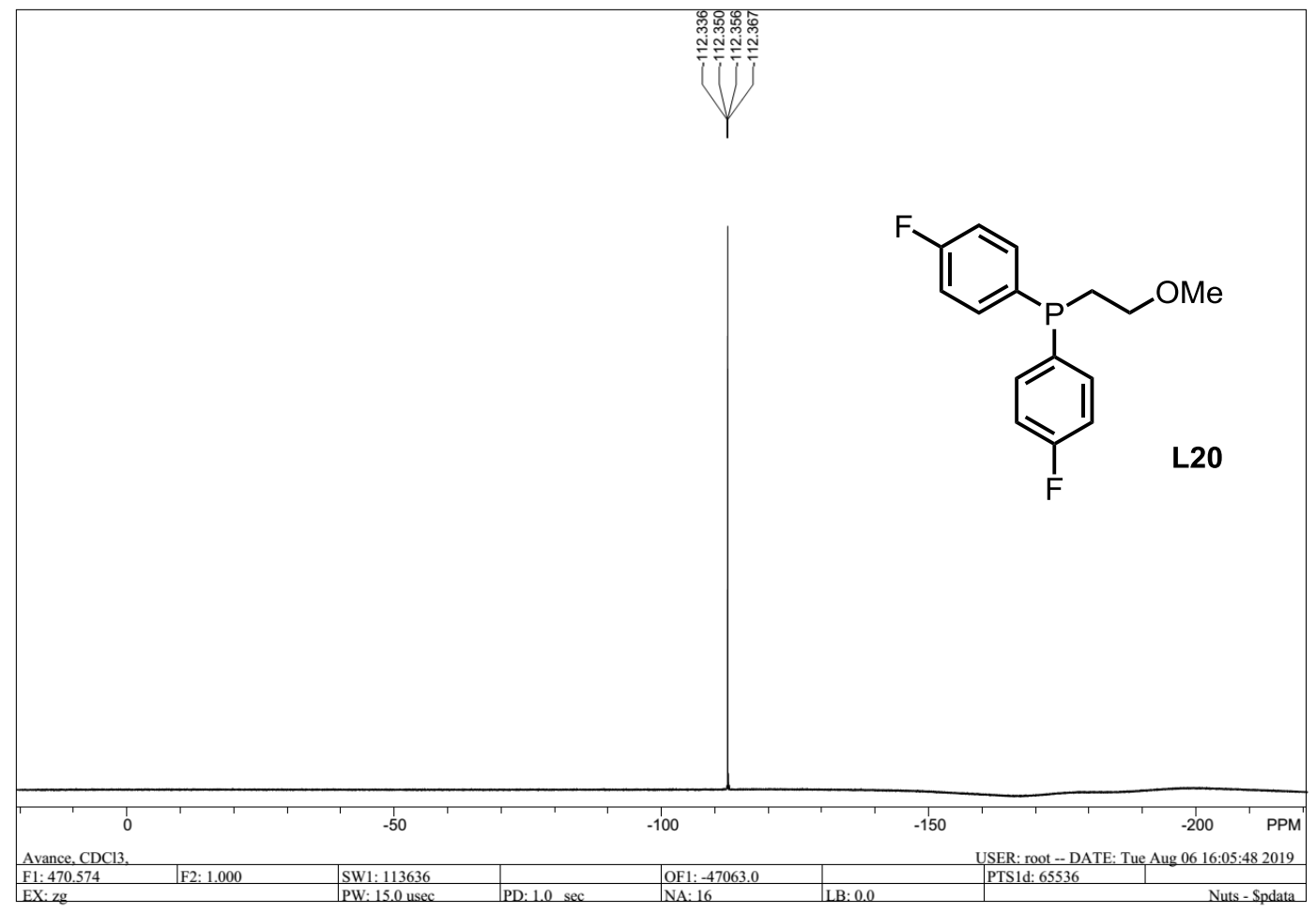

${ }^{31}$ P NMR (202 MHz, $\left.\mathrm{CDCl}_{3}\right)$ :

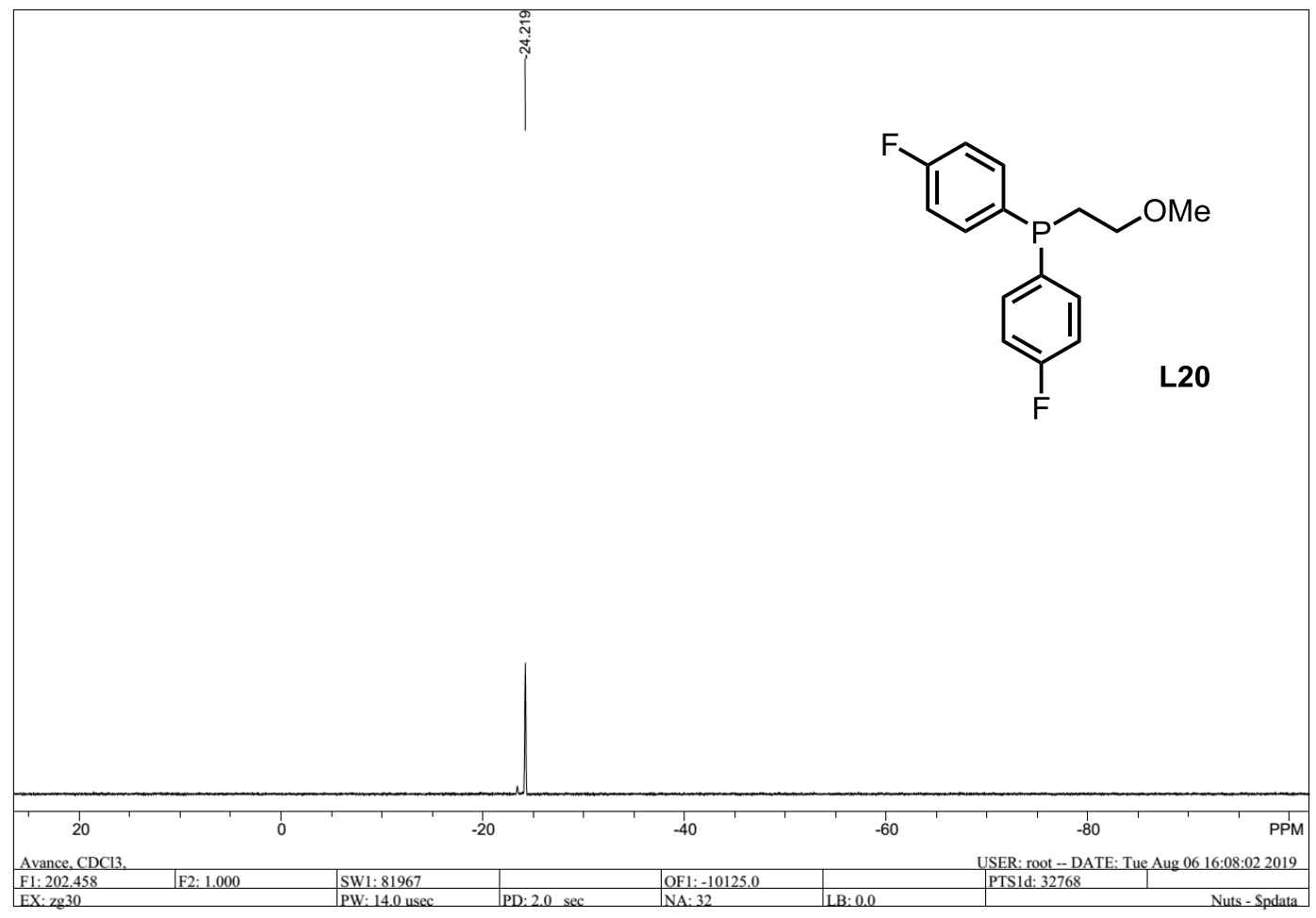


${ }^{1} \mathrm{H}$ NMR (500MHz, $\left.\mathrm{CDCl}_{3}\right)$ :

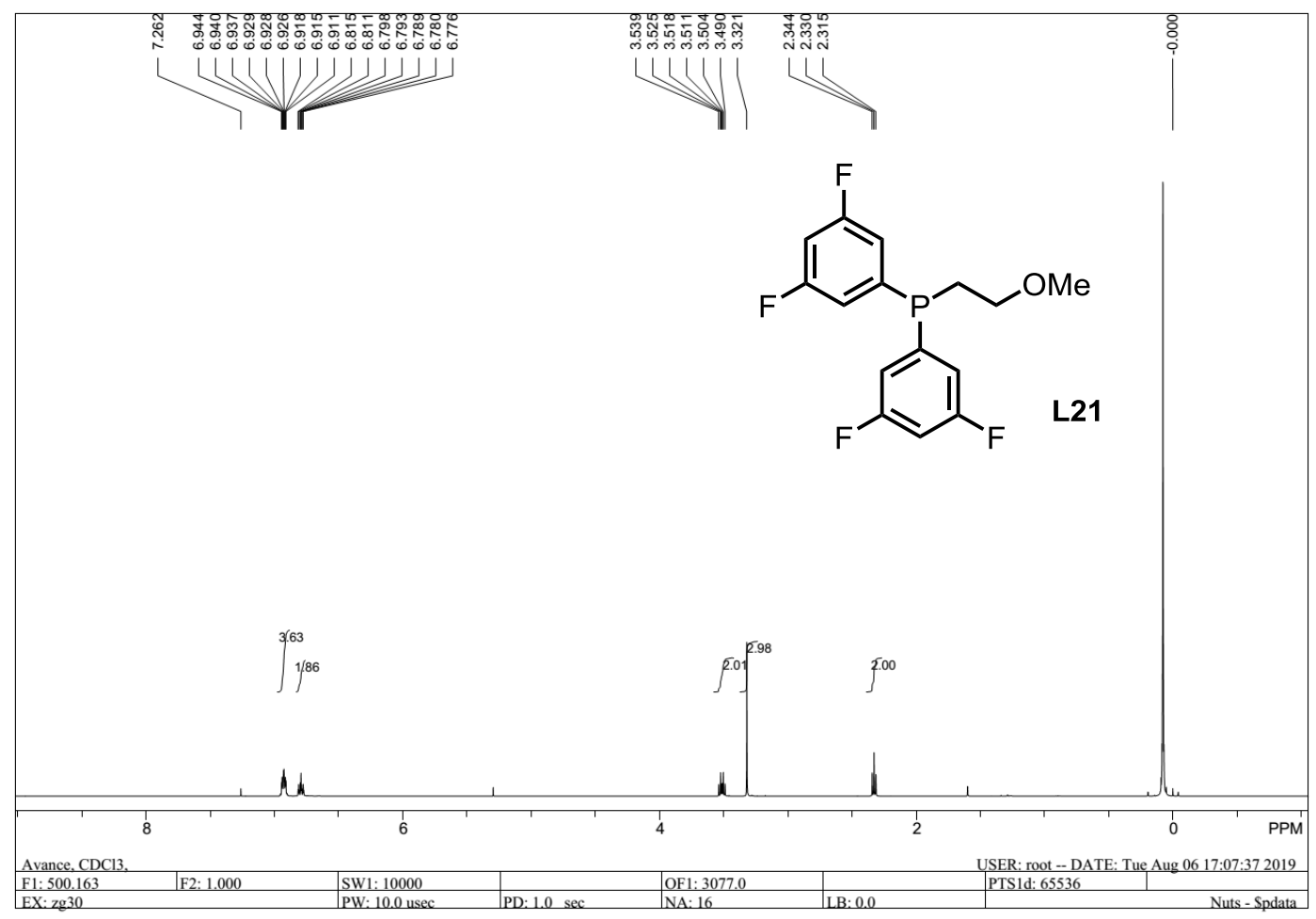

${ }^{13} \mathrm{C}$ NMR $\left(125 \mathrm{MHz}, \mathrm{CDCl}_{3}\right)$ :

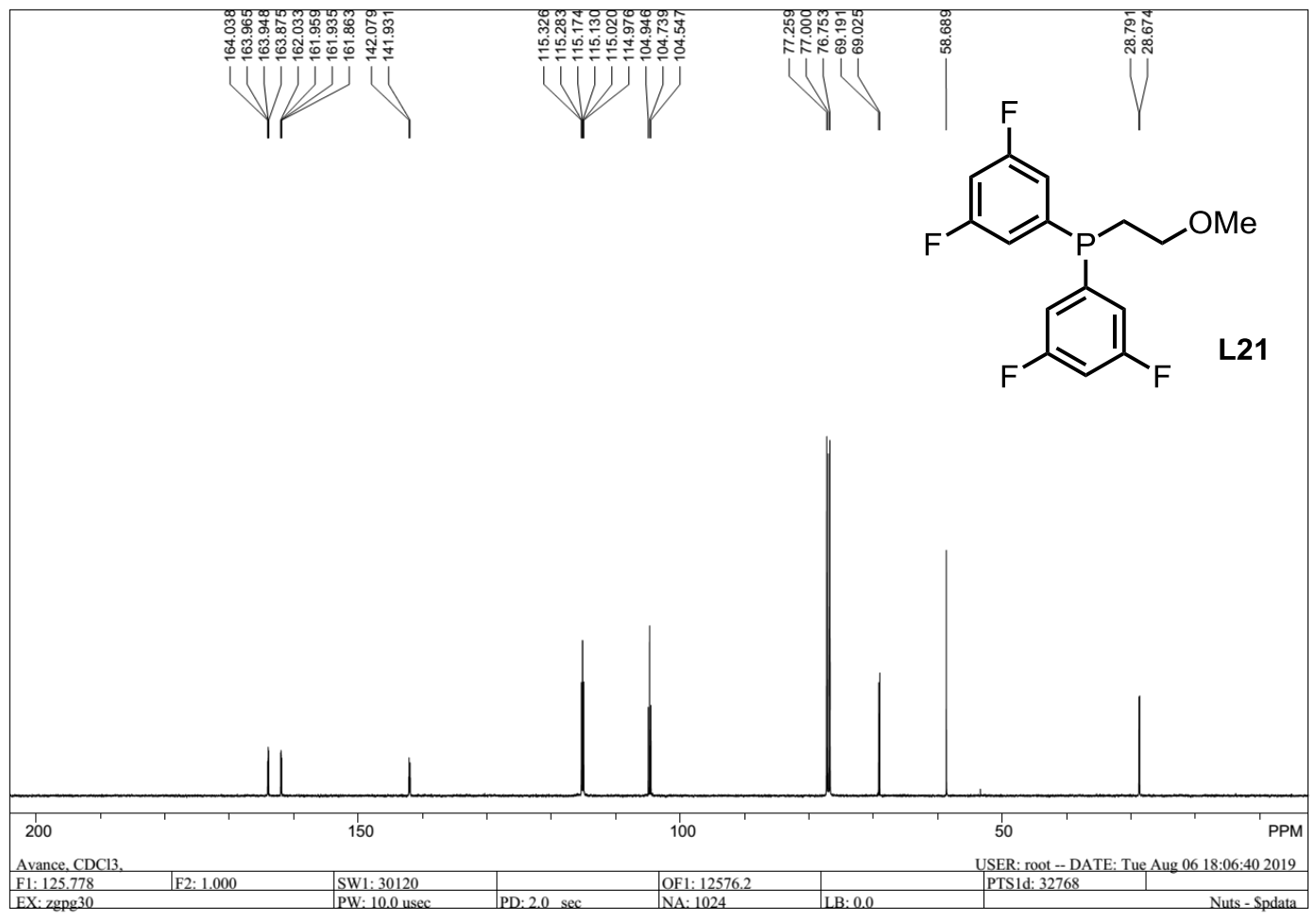


${ }^{19} \mathrm{~F}$ NMR (470 MHz, $\mathrm{CDCl}_{3}$ ):

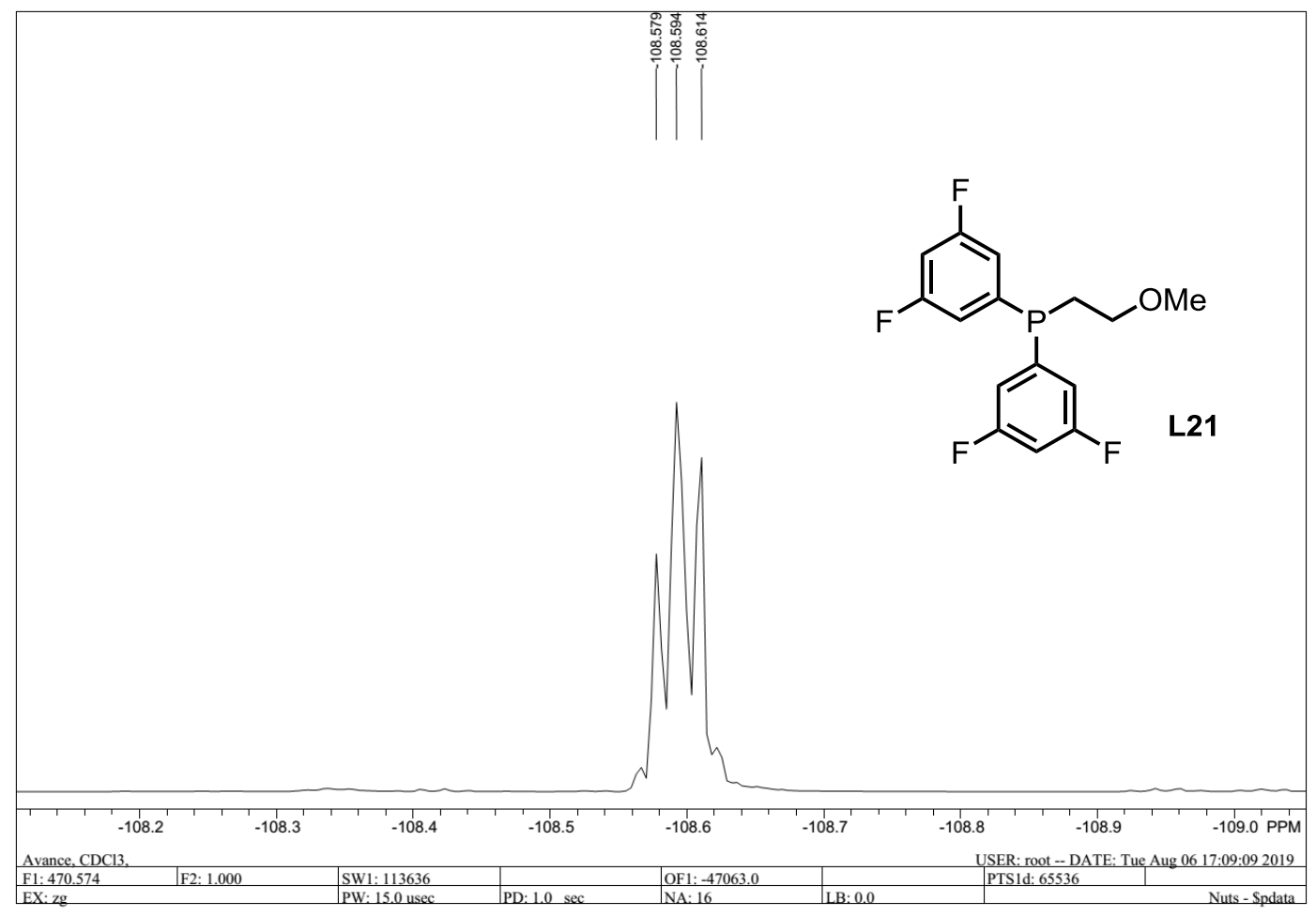

${ }^{31}$ P NMR (202 MHz, $\left.\mathrm{CDCl}_{3}\right)$ :

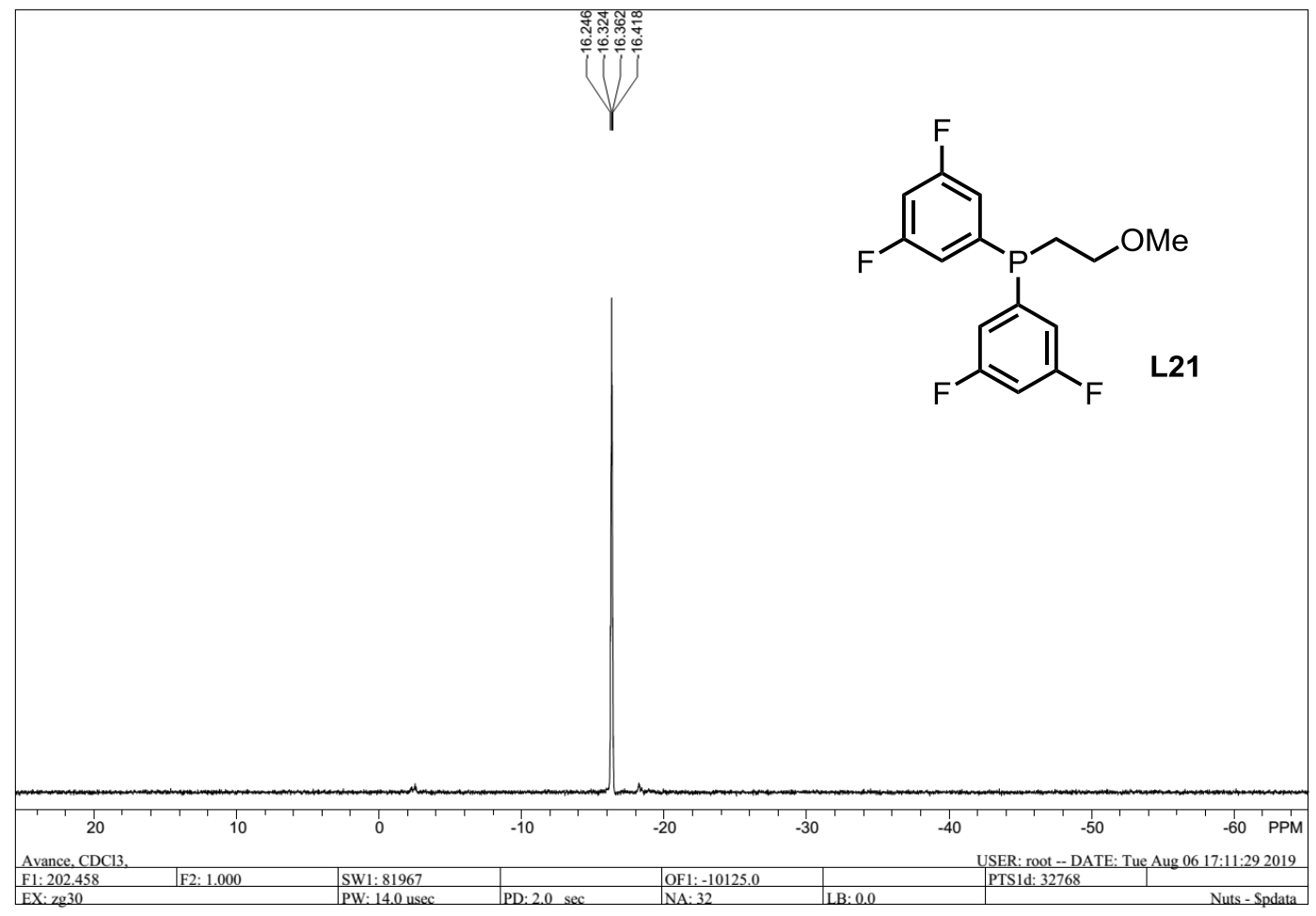


${ }^{1} \mathrm{H}$ NMR $\left(500 \mathrm{MHz}, \mathrm{CDCl}_{3}\right)$ :

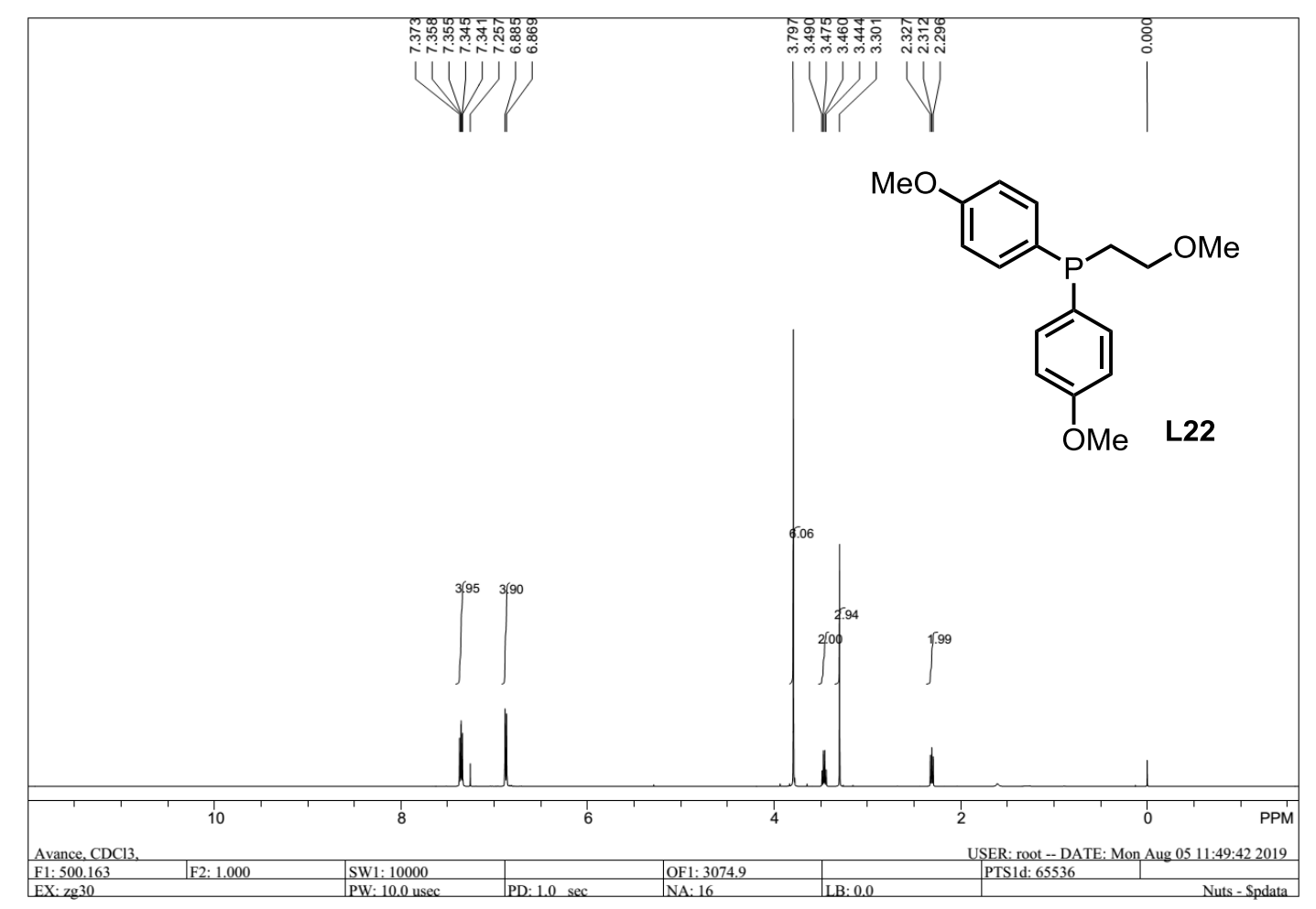

${ }^{13} \mathrm{C}$ NMR (125MHz, $\left.\mathrm{CDCl}_{3}\right)$ :

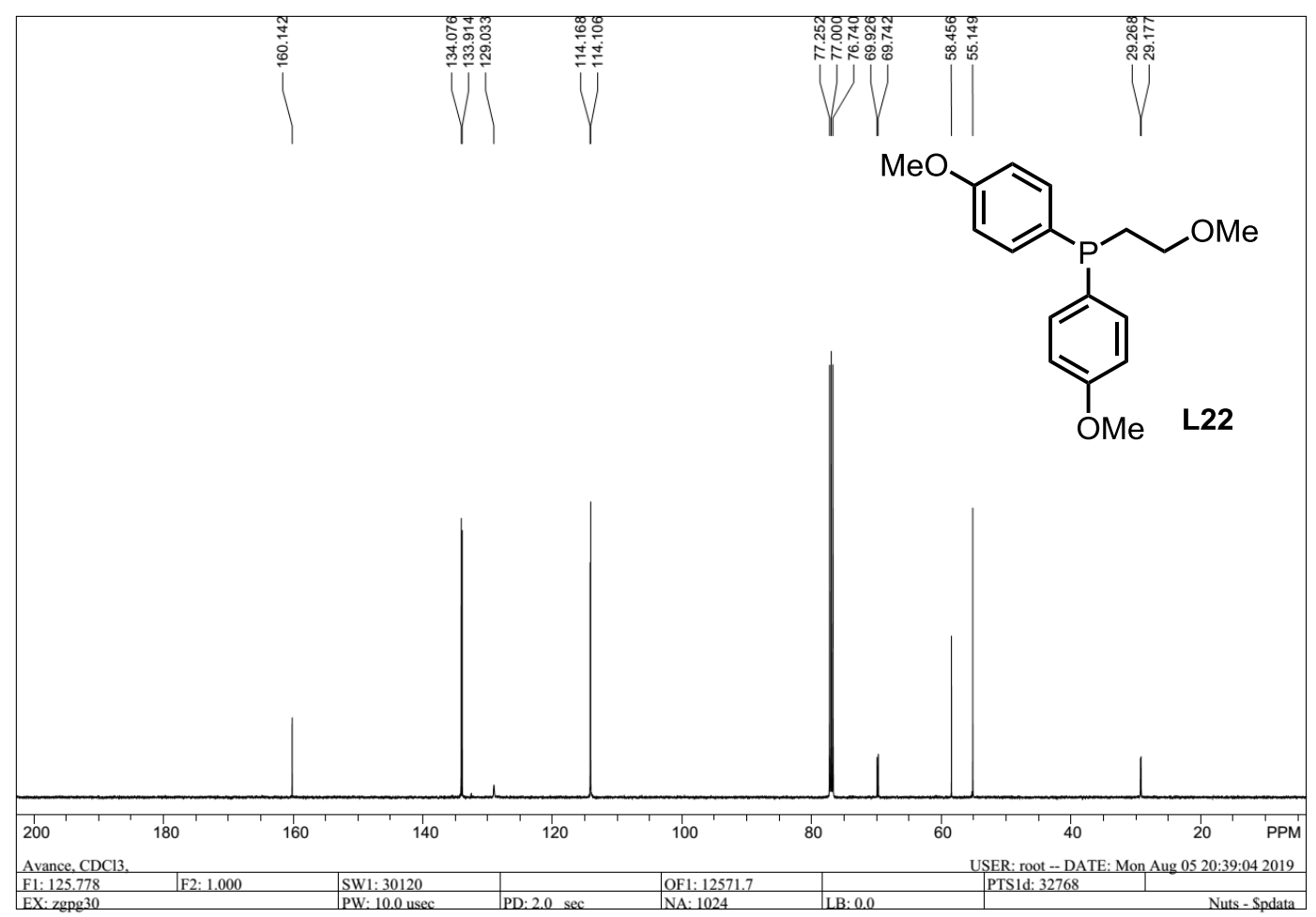


${ }^{31}$ P NMR (202 MHz, $\left.\mathrm{CDCl}_{3}\right)$ :

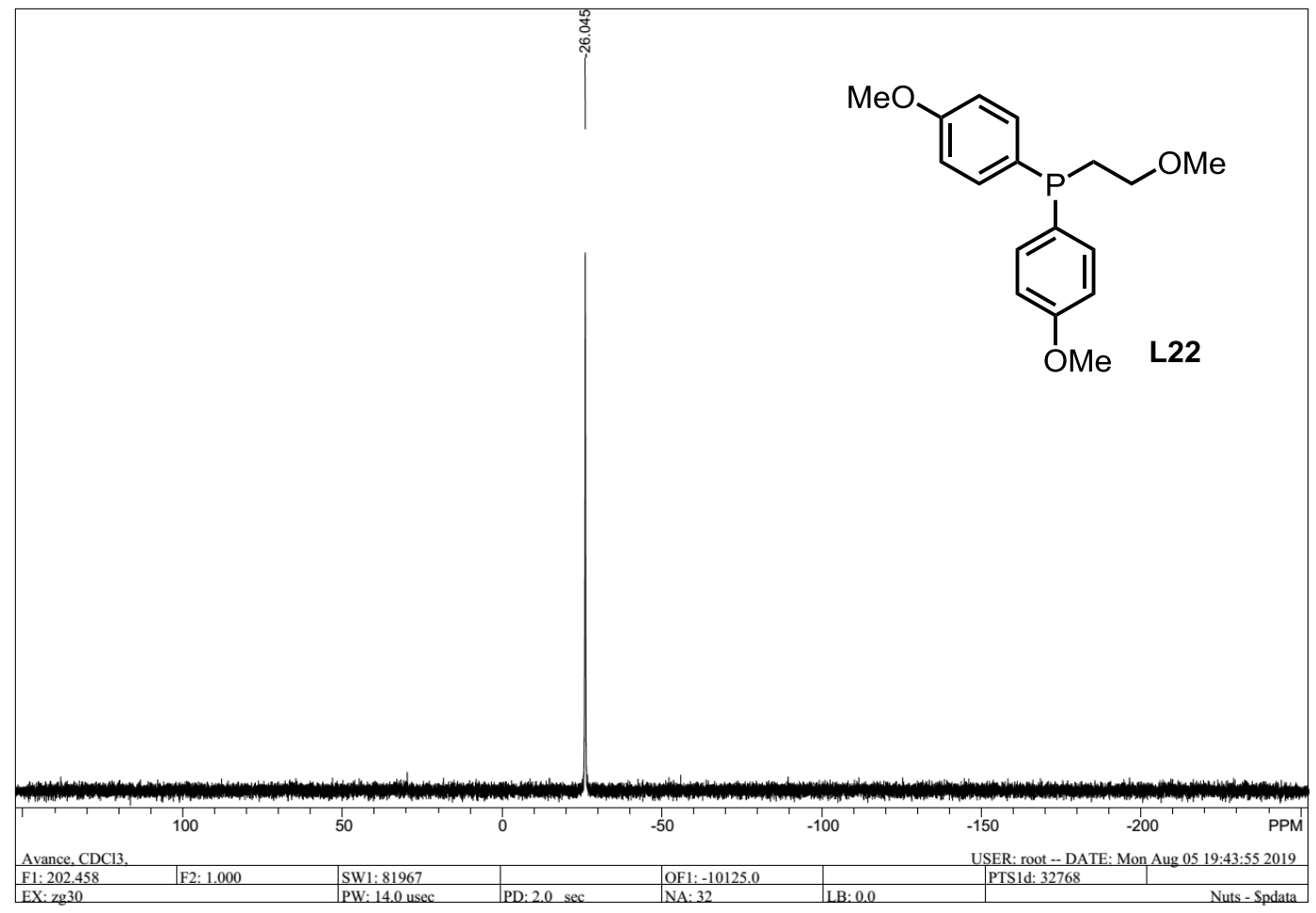


${ }^{1} \mathrm{H}$ NMR $\left(500 \mathrm{MHz}, \mathrm{CDCl}_{3}\right)$ :

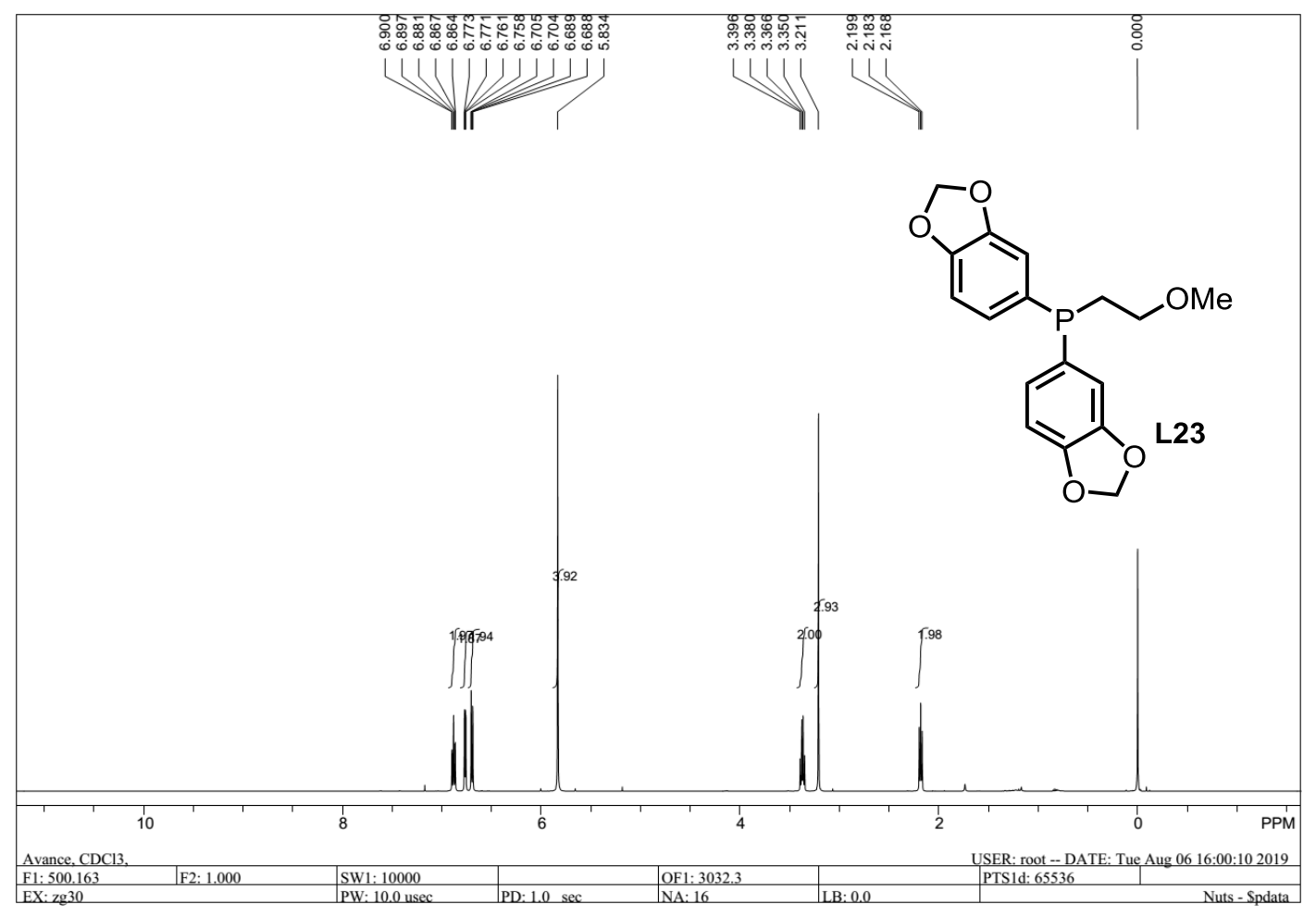

${ }^{13} \mathrm{C}$ NMR $\left(125 \mathrm{MHz}, \mathrm{CDCl}_{3}\right)$ :

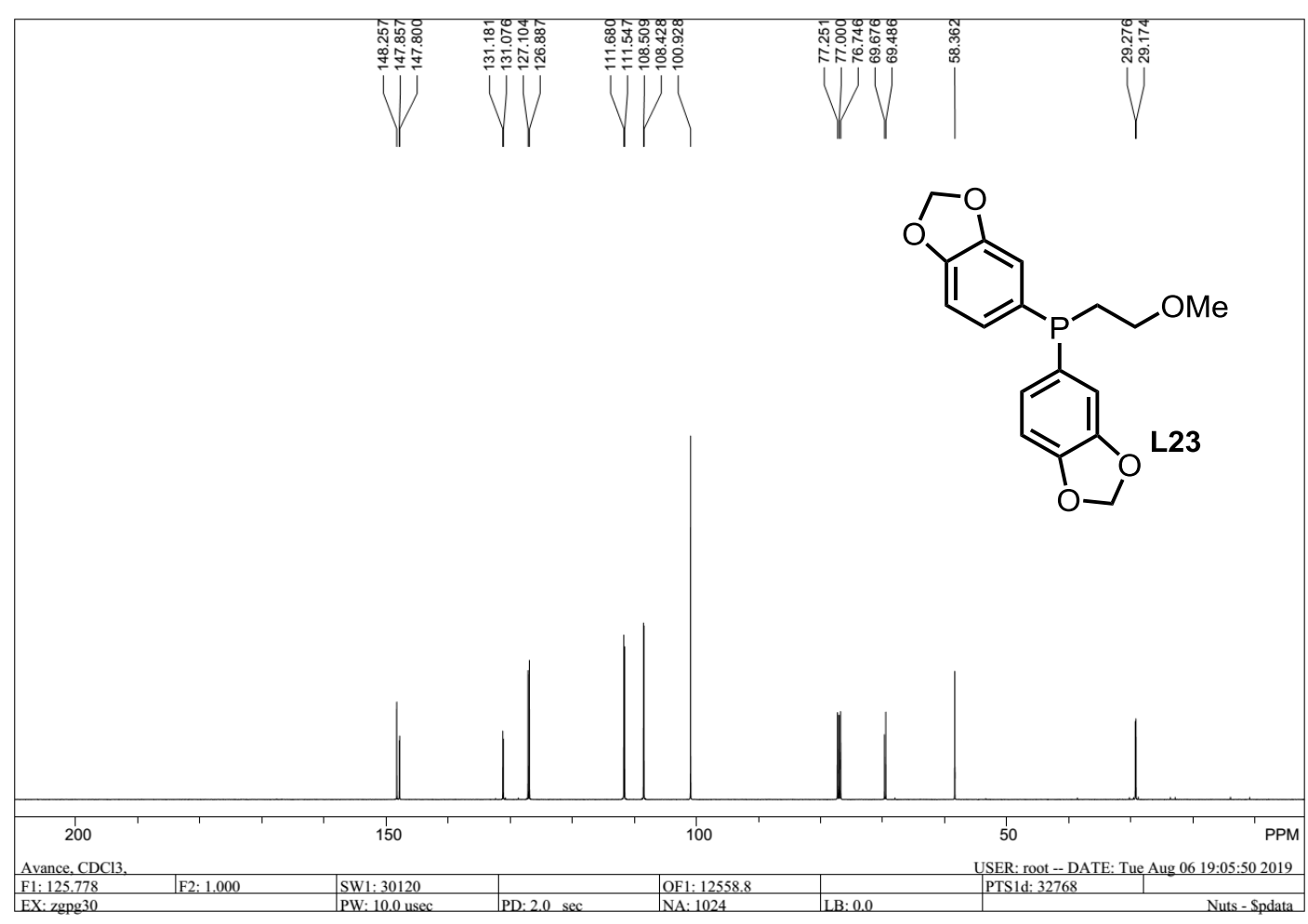


${ }^{31}$ P NMR (202 MHz, $\left.\mathrm{CDCl}_{3}\right)$ :

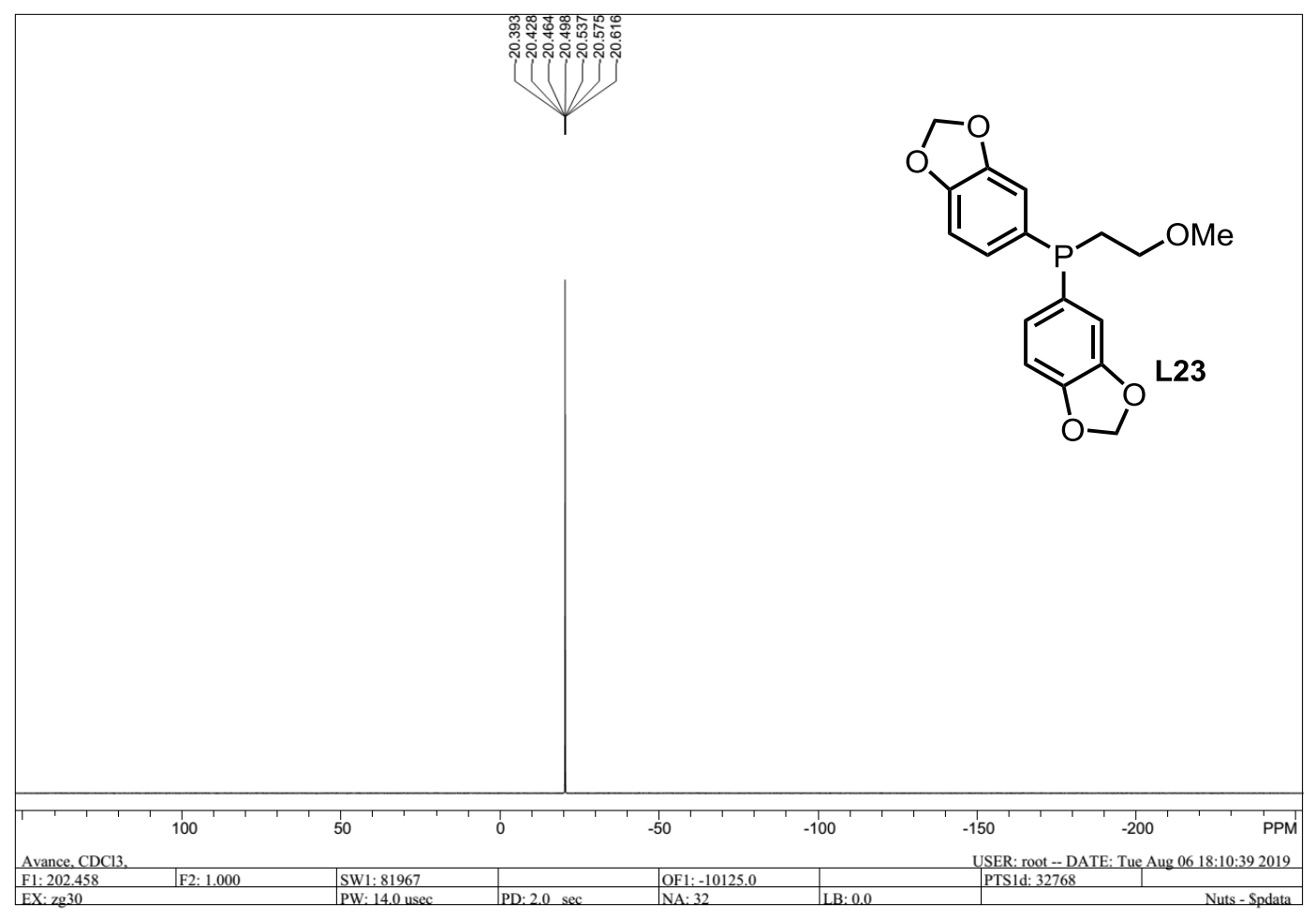


${ }^{1} \mathrm{H}$ NMR (500MHz, $\left.\mathrm{CDCl}_{3}\right)$ :

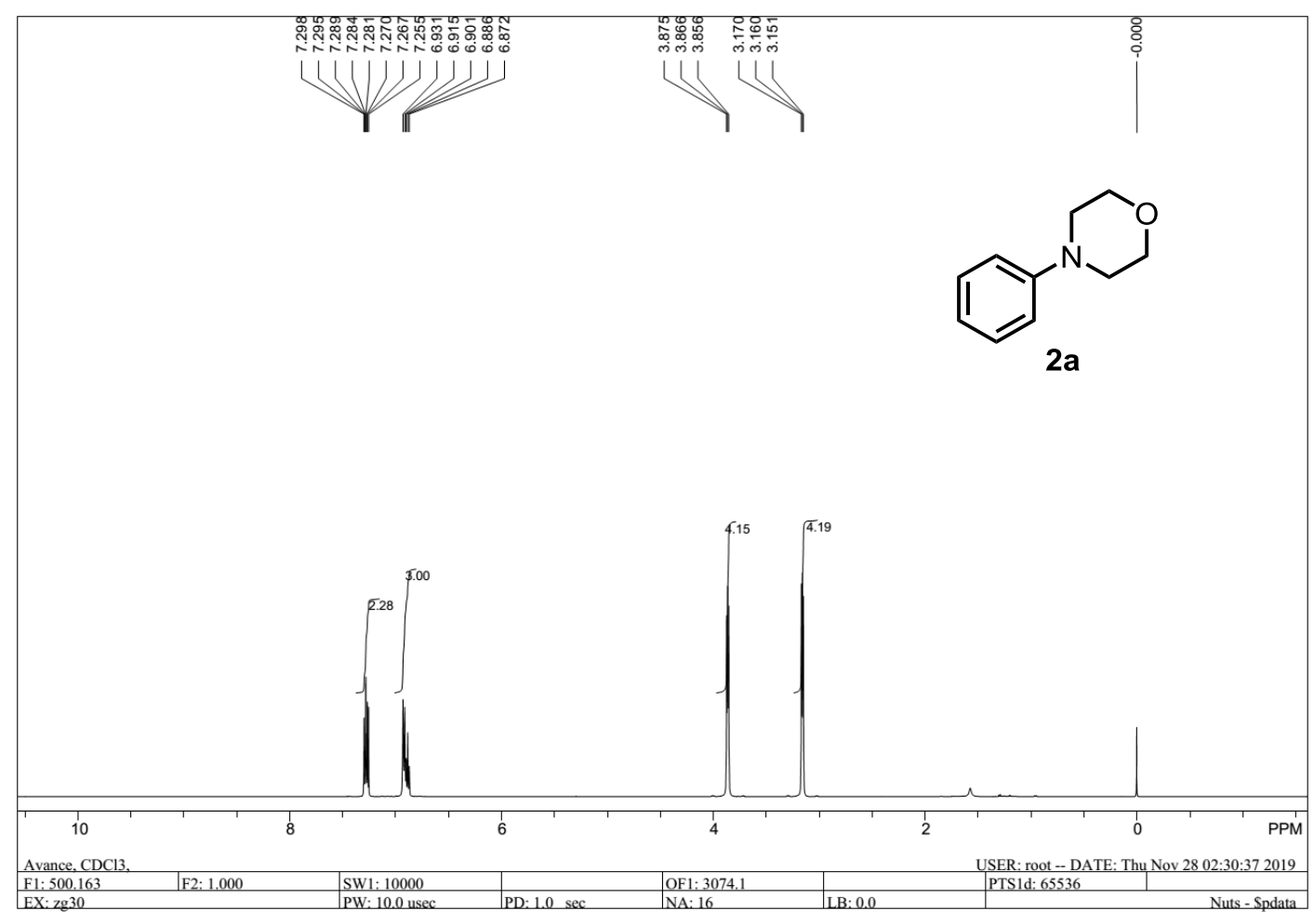

${ }^{1} \mathrm{H}$ NMR (500MHz, $\left.\mathrm{CDCl}_{3}\right)$ :

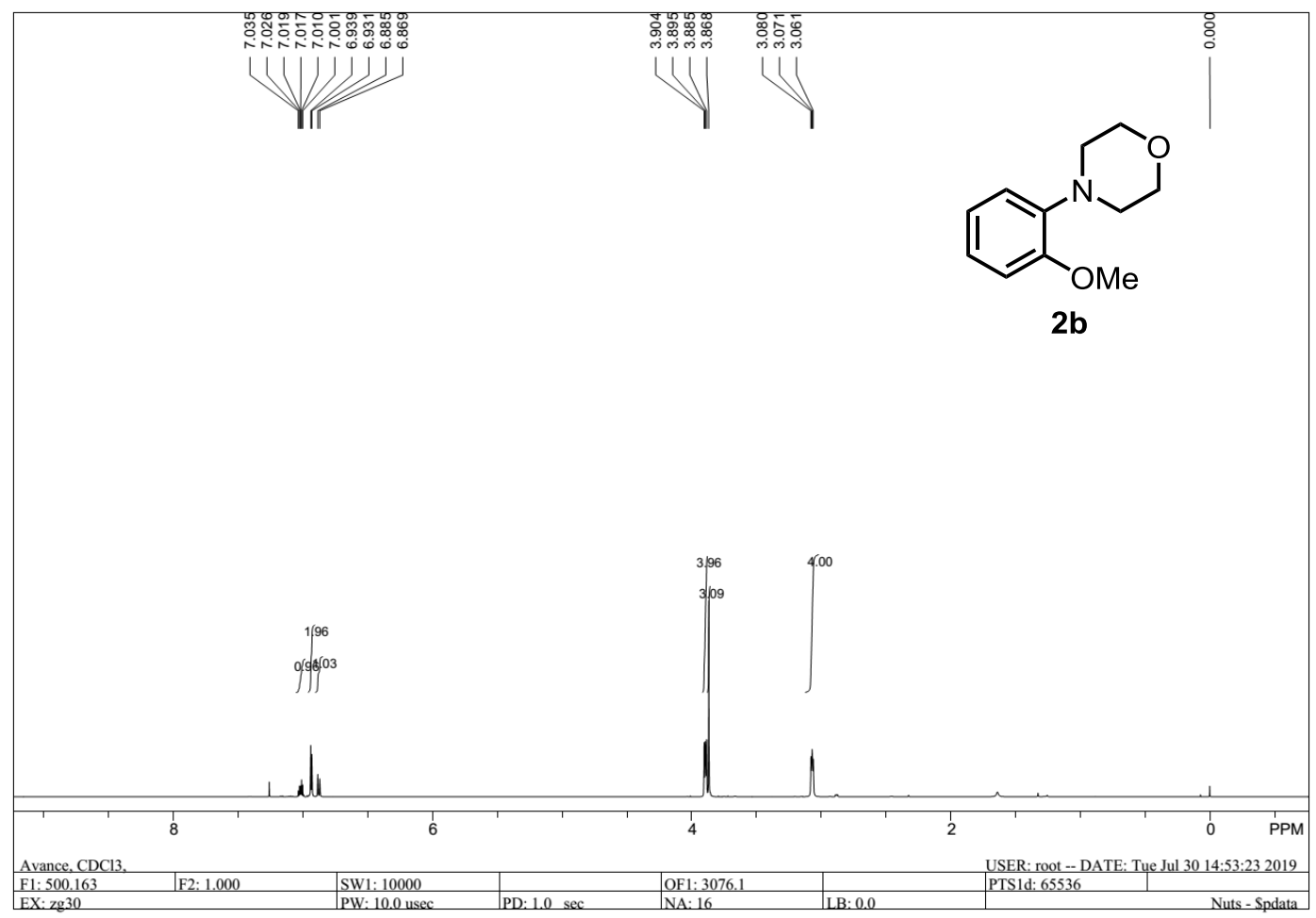


${ }^{1} \mathrm{H}$ NMR (500MHz, $\mathrm{CDCl}_{3}$ ):

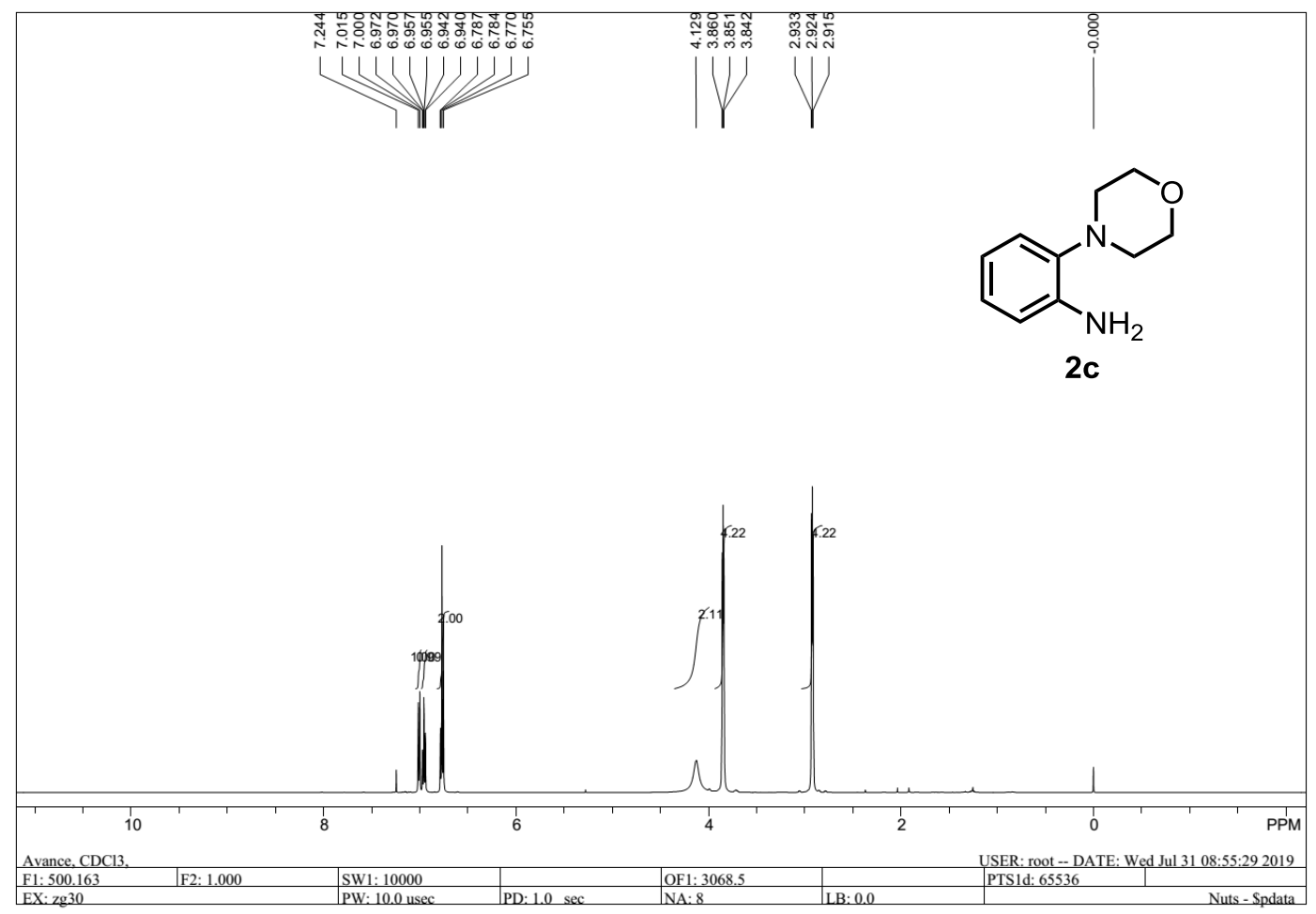

${ }^{1} \mathrm{H} \mathrm{NMR}\left(500 \mathrm{MHz}, \mathrm{CDCl}_{3}\right)$ :

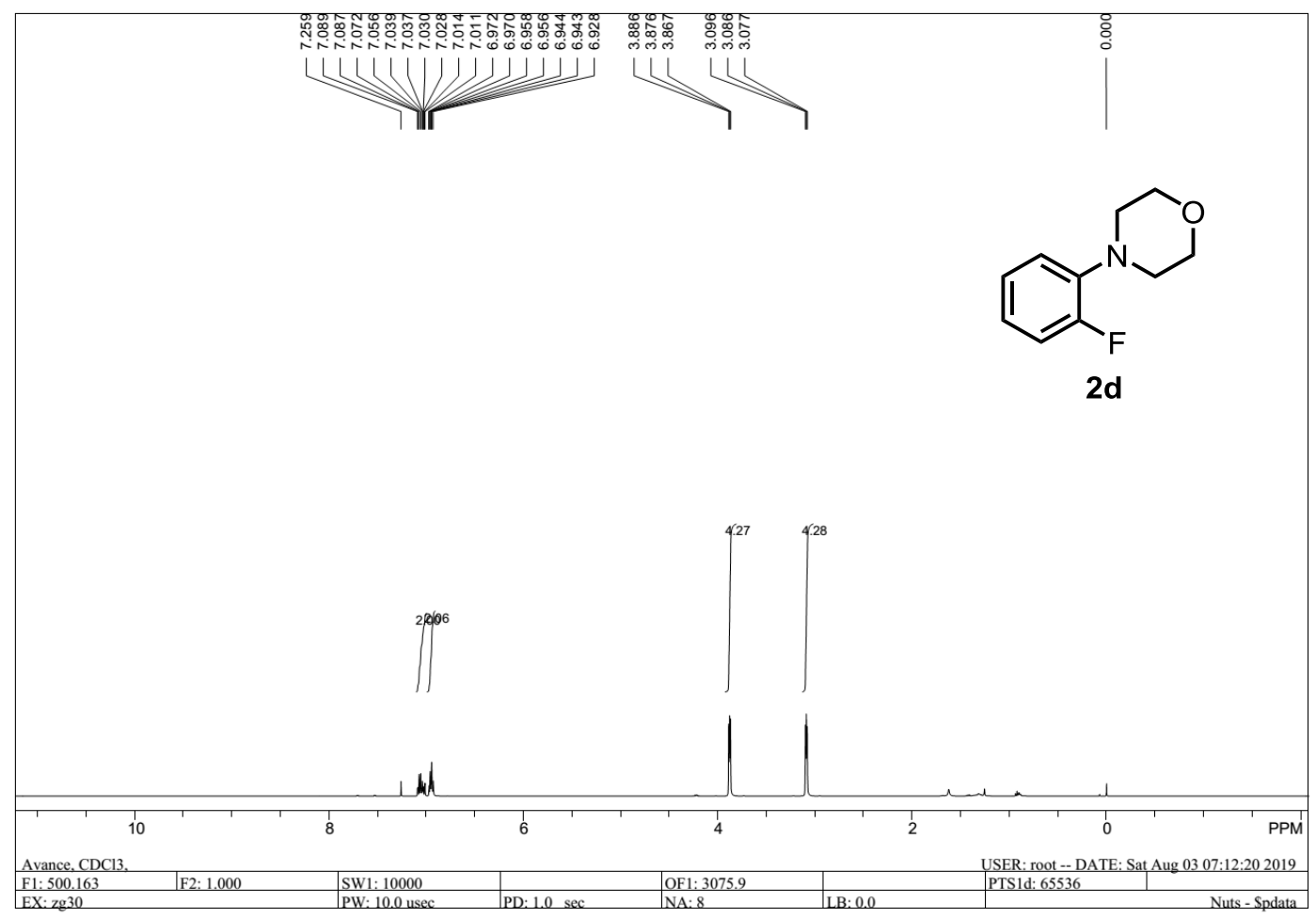


${ }^{1} \mathrm{H}$ NMR (500MHz, $\mathrm{CDCl}_{3}$ ):

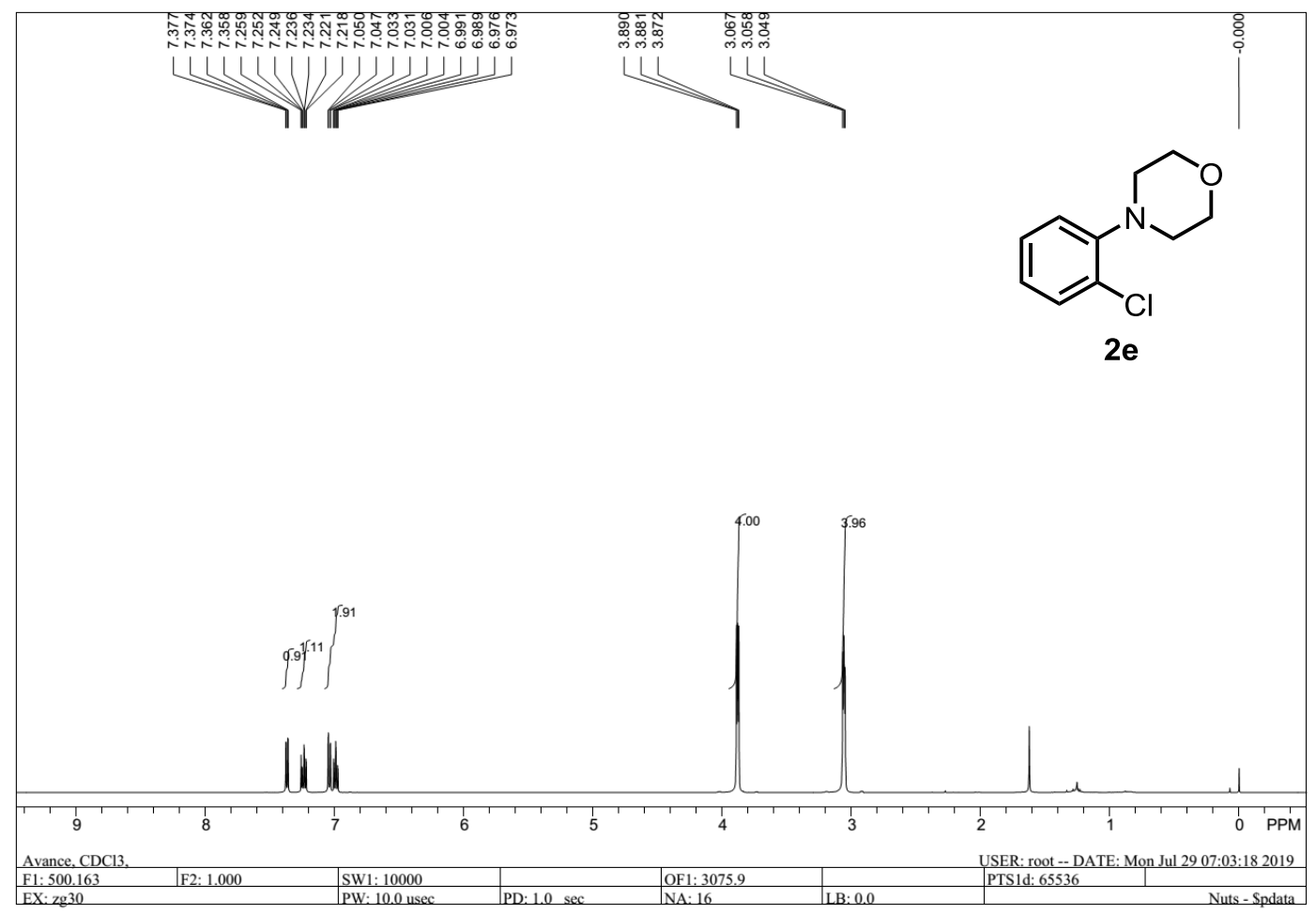

${ }^{1} \mathrm{H}$ NMR (500MHz, $\mathrm{CDCl}_{3}$ ):

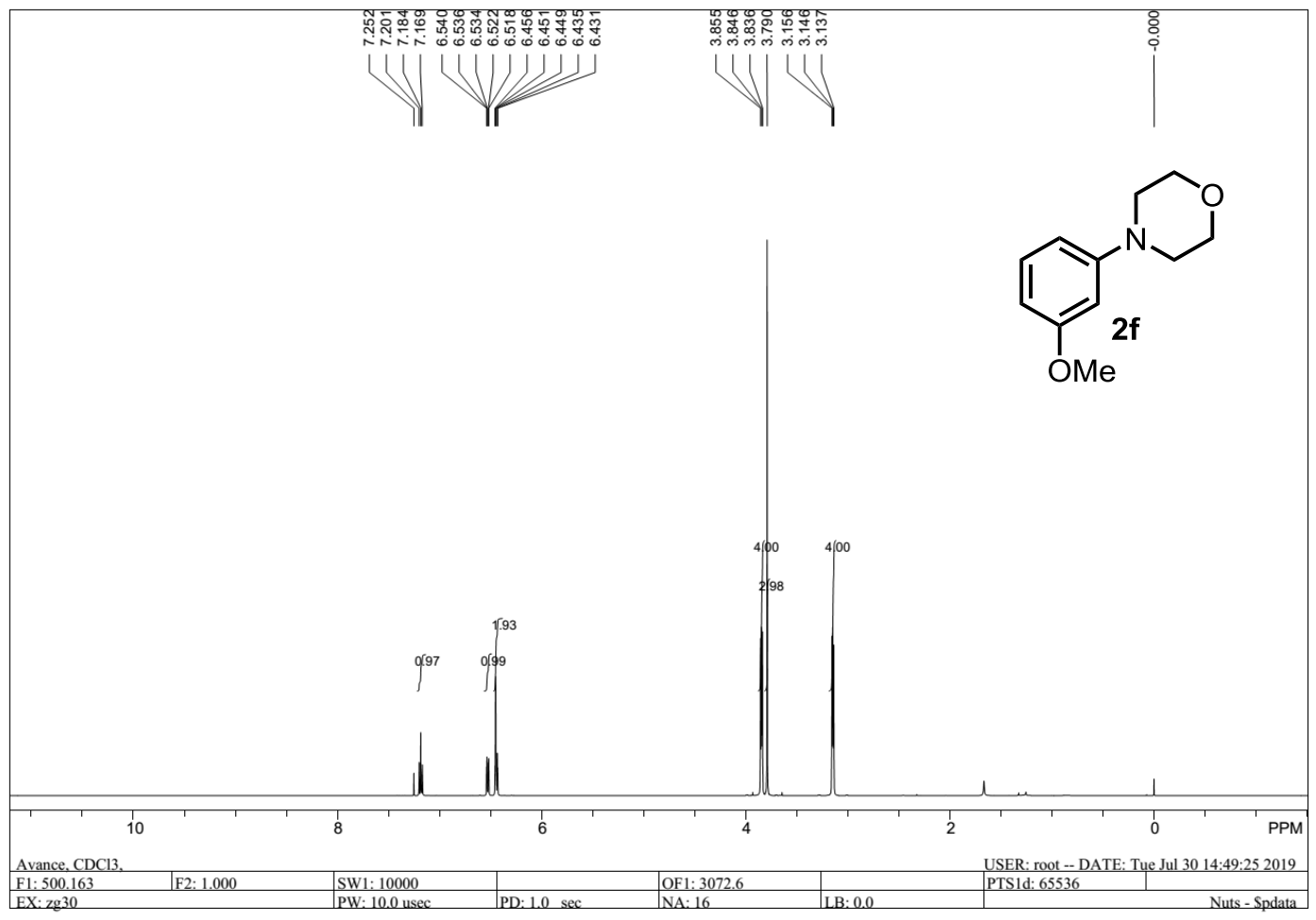


${ }^{1} \mathrm{H}$ NMR (500MHz, $\left.\mathrm{CDCl}_{3}\right)$ :

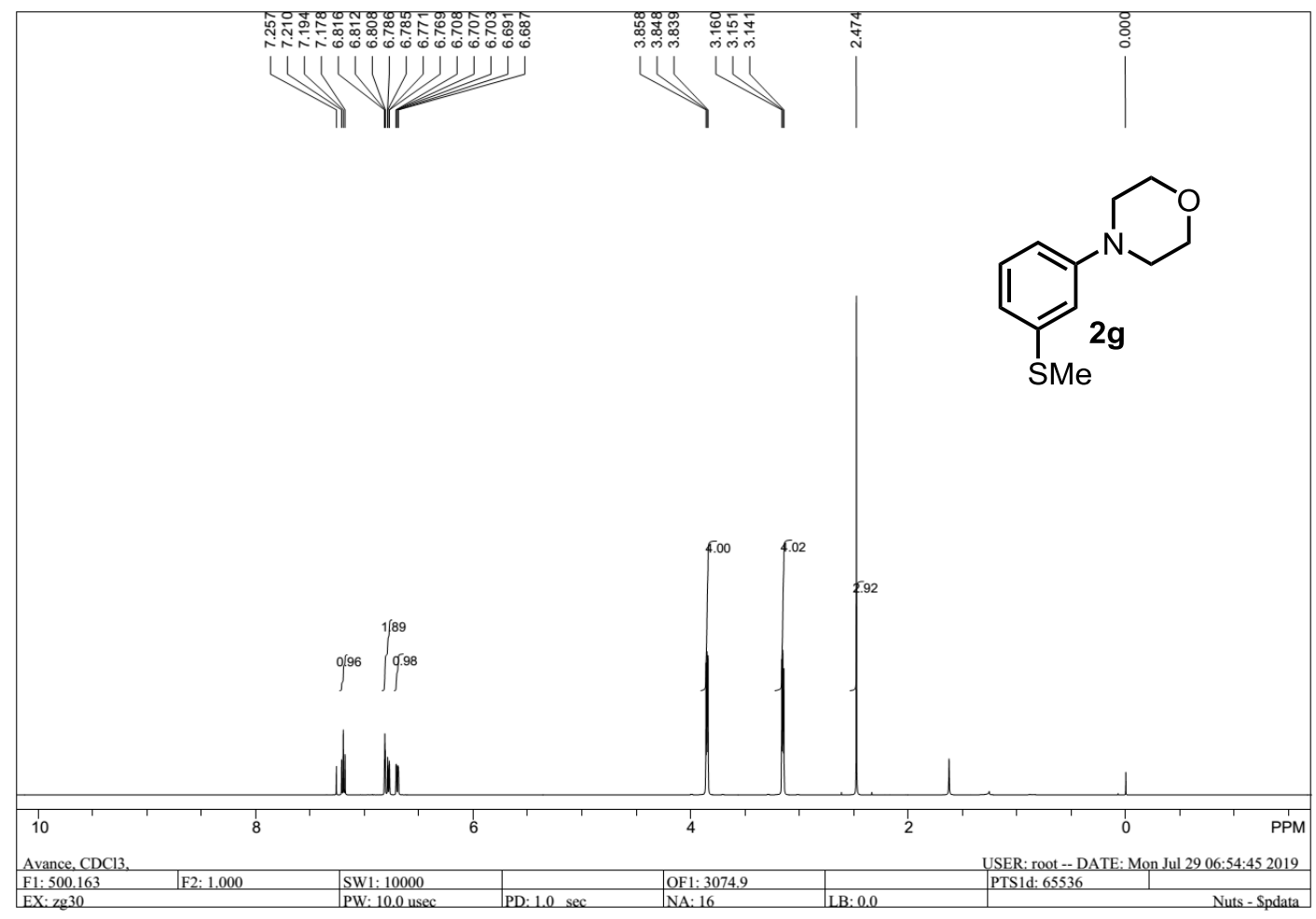

${ }^{1} \mathrm{H}$ NMR (500MHz, $\left.\mathrm{CDCl}_{3}\right)$ :

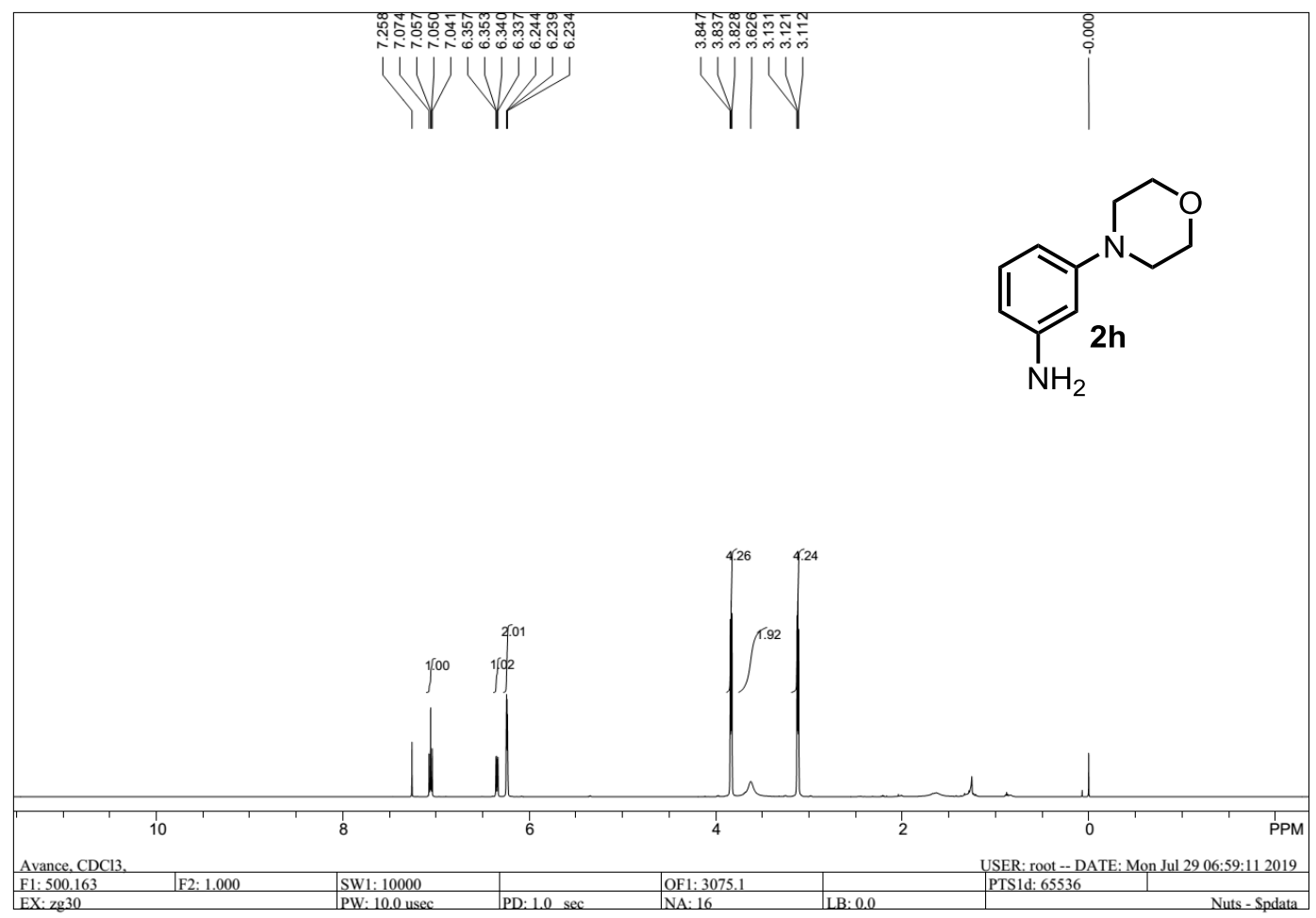


${ }^{1} \mathrm{H}$ NMR $\left(500 \mathrm{MHz}, \mathrm{CDCl}_{3}\right)$ :

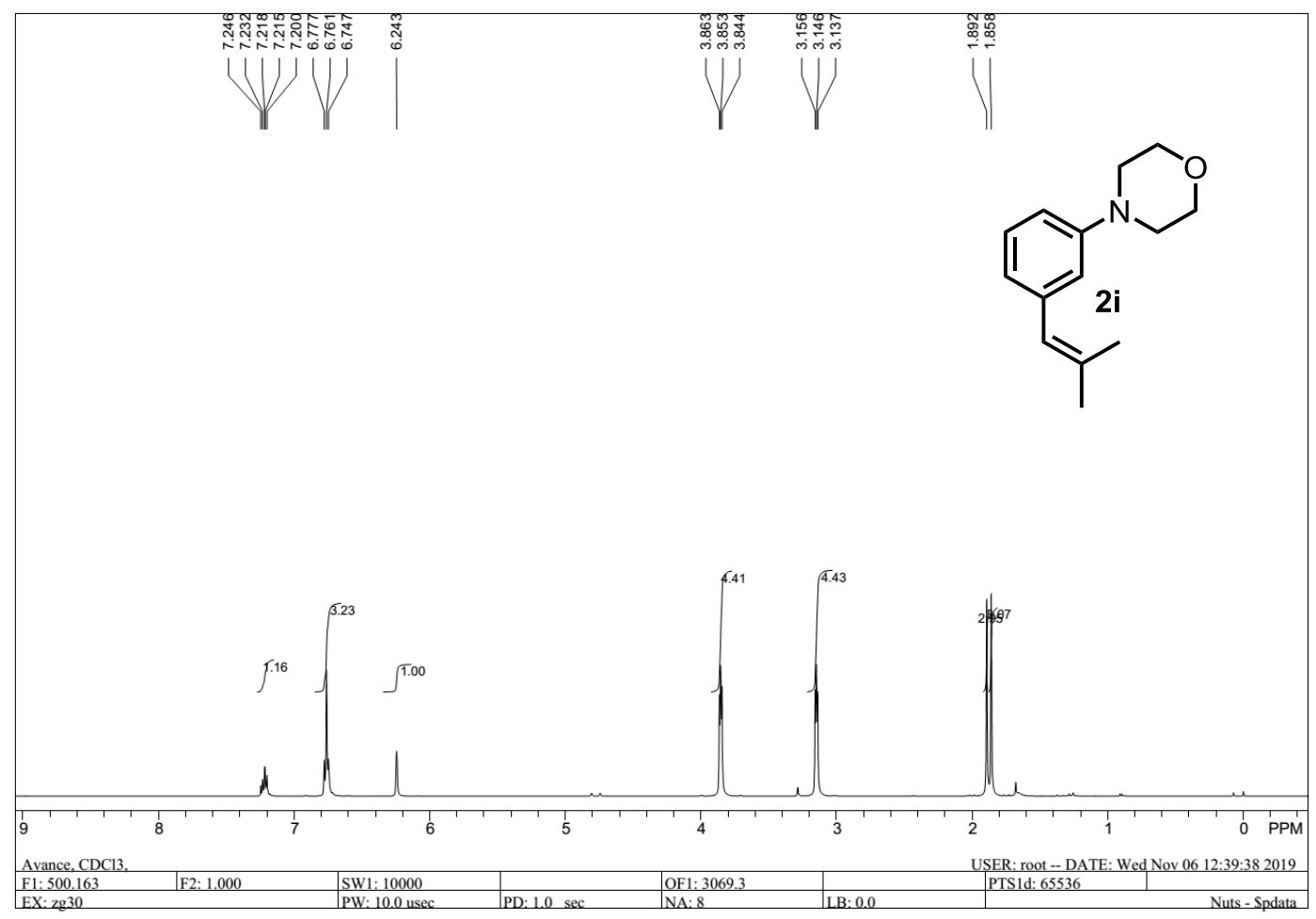

${ }^{13} \mathrm{C}$ NMR $\left(125 \mathrm{MHz}, \mathrm{CDCl}_{3}\right)$ :

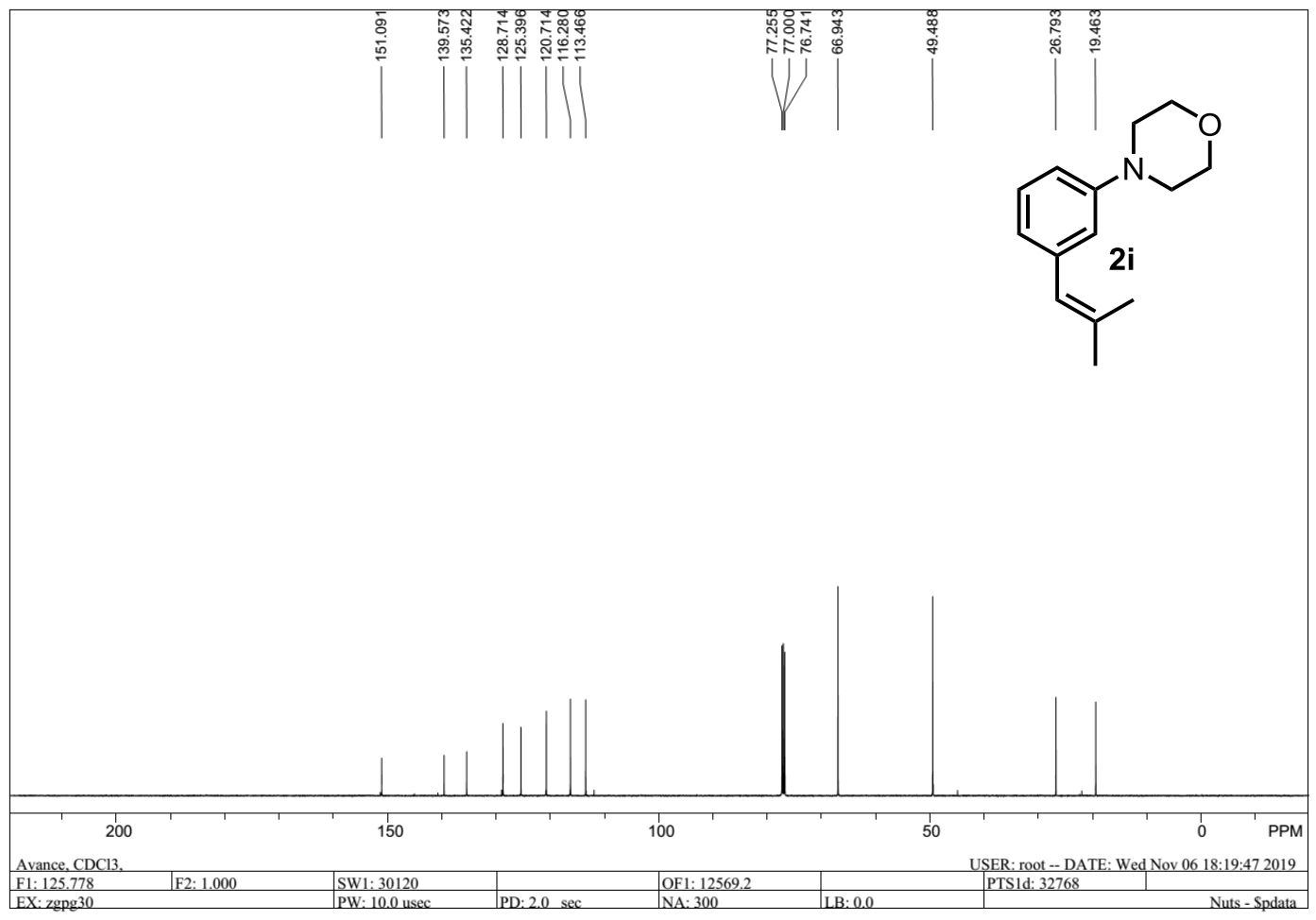


${ }^{1} \mathrm{H}$ NMR $\left(500 \mathrm{MHz}, \mathrm{CDCl}_{3}\right)$ :

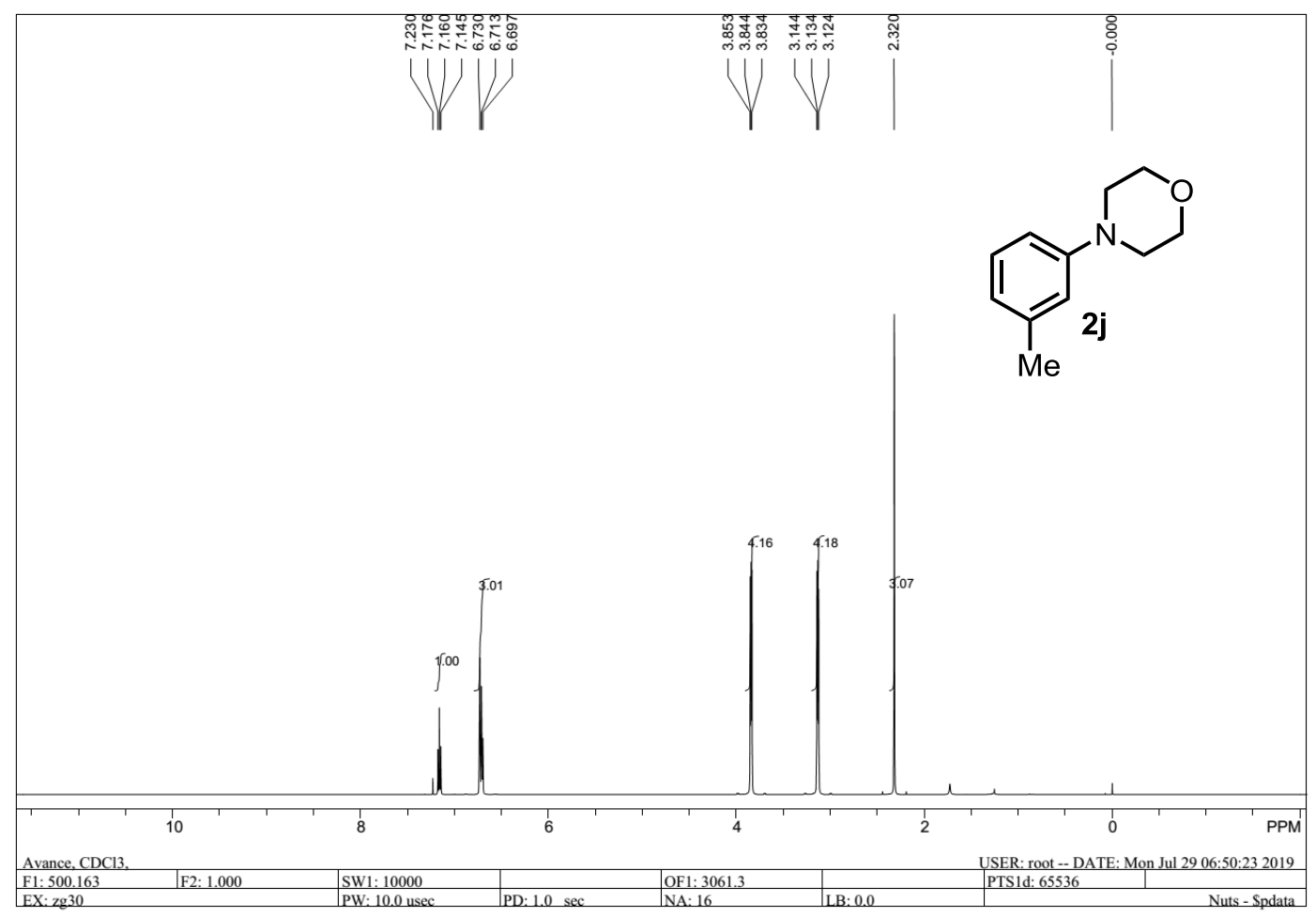


${ }^{1} \mathrm{H}$ NMR $\left(500 \mathrm{MHz}, \mathrm{CDCl}_{3}\right)$ :

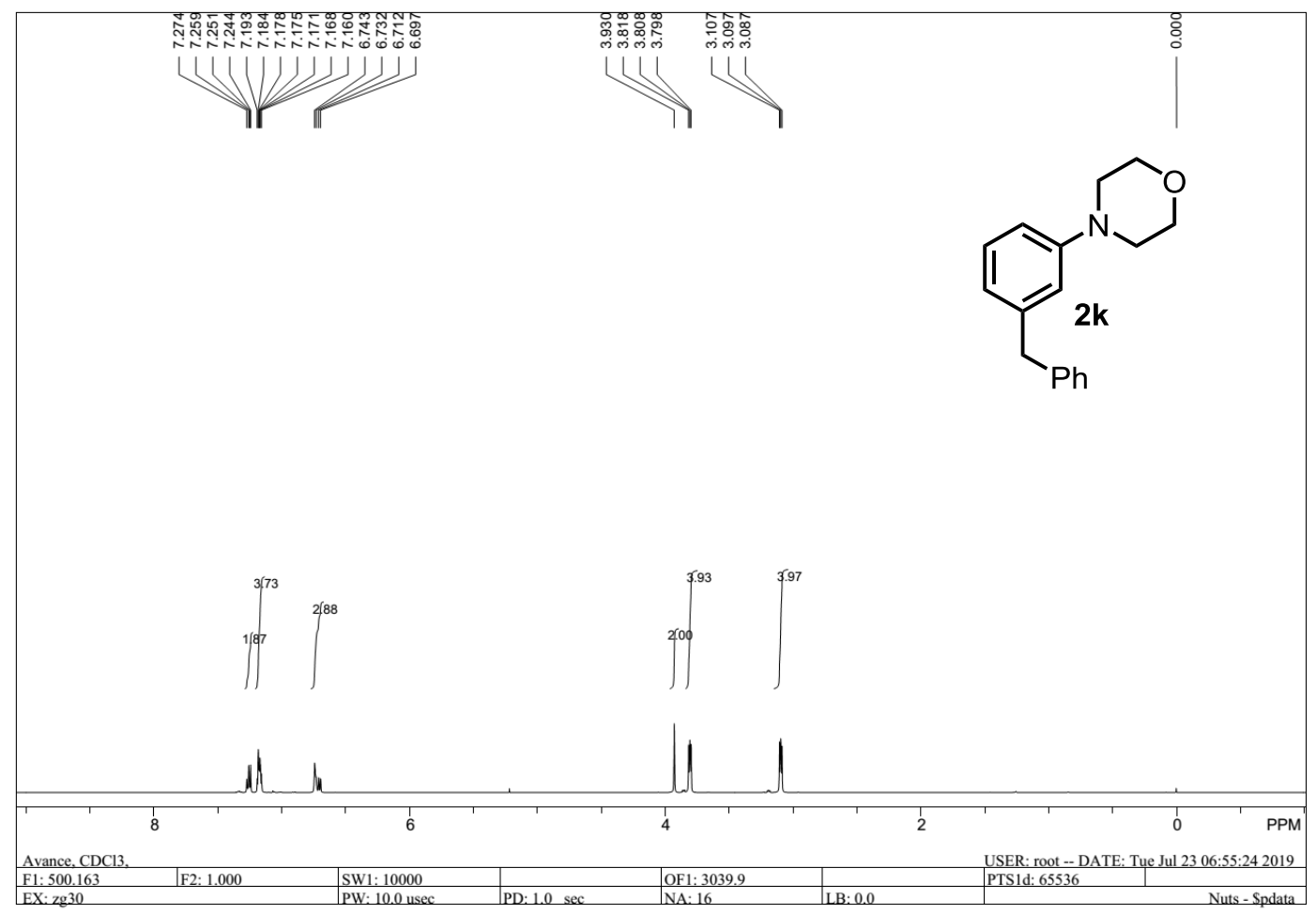

${ }^{13} \mathrm{C}$ NMR $\left(125 \mathrm{MHz}, \mathrm{CDCl}_{3}\right)$ :

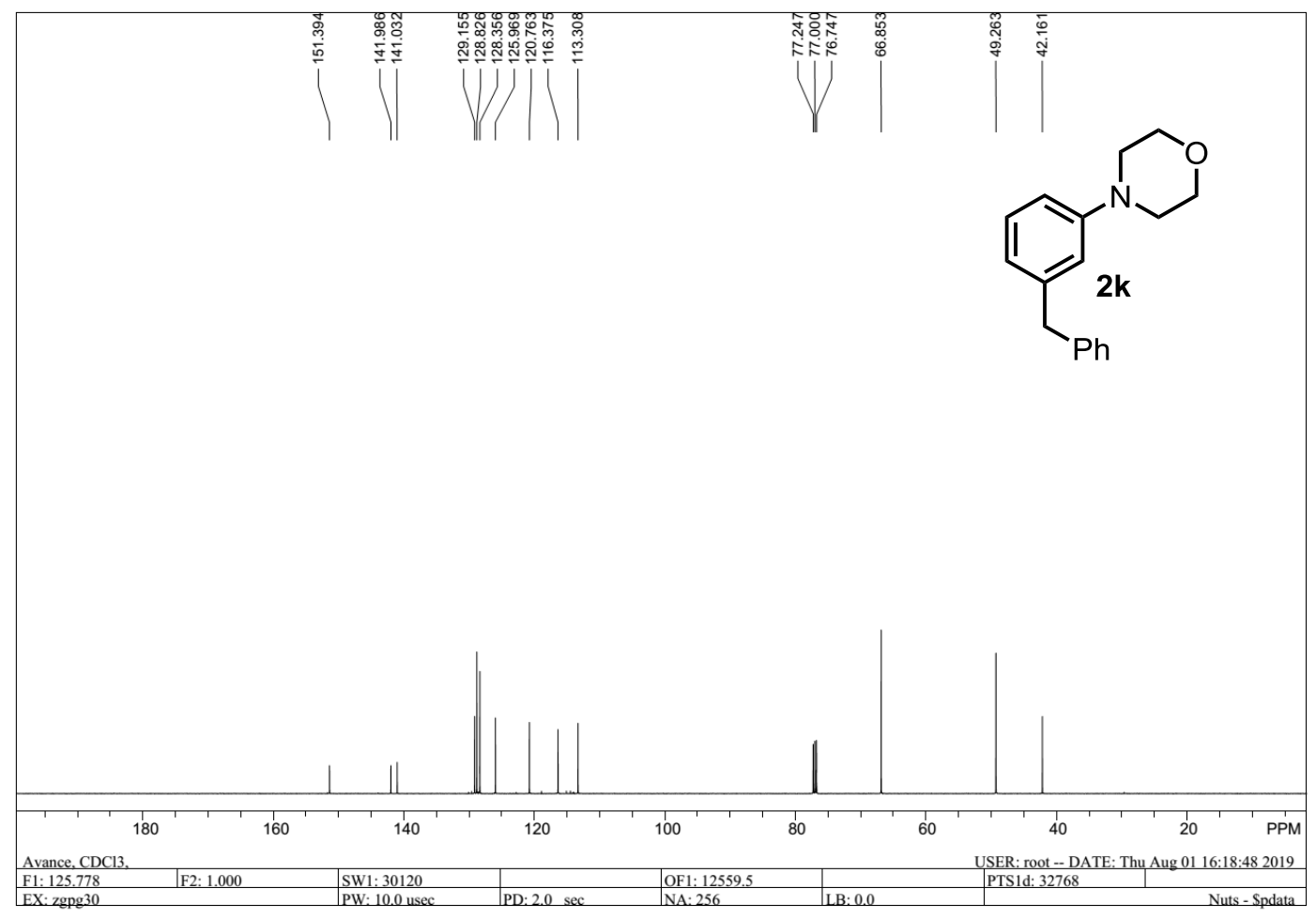


${ }^{1} \mathrm{H}$ NMR $\left(500 \mathrm{MHz}, \mathrm{CDCl}_{3}\right)$ :

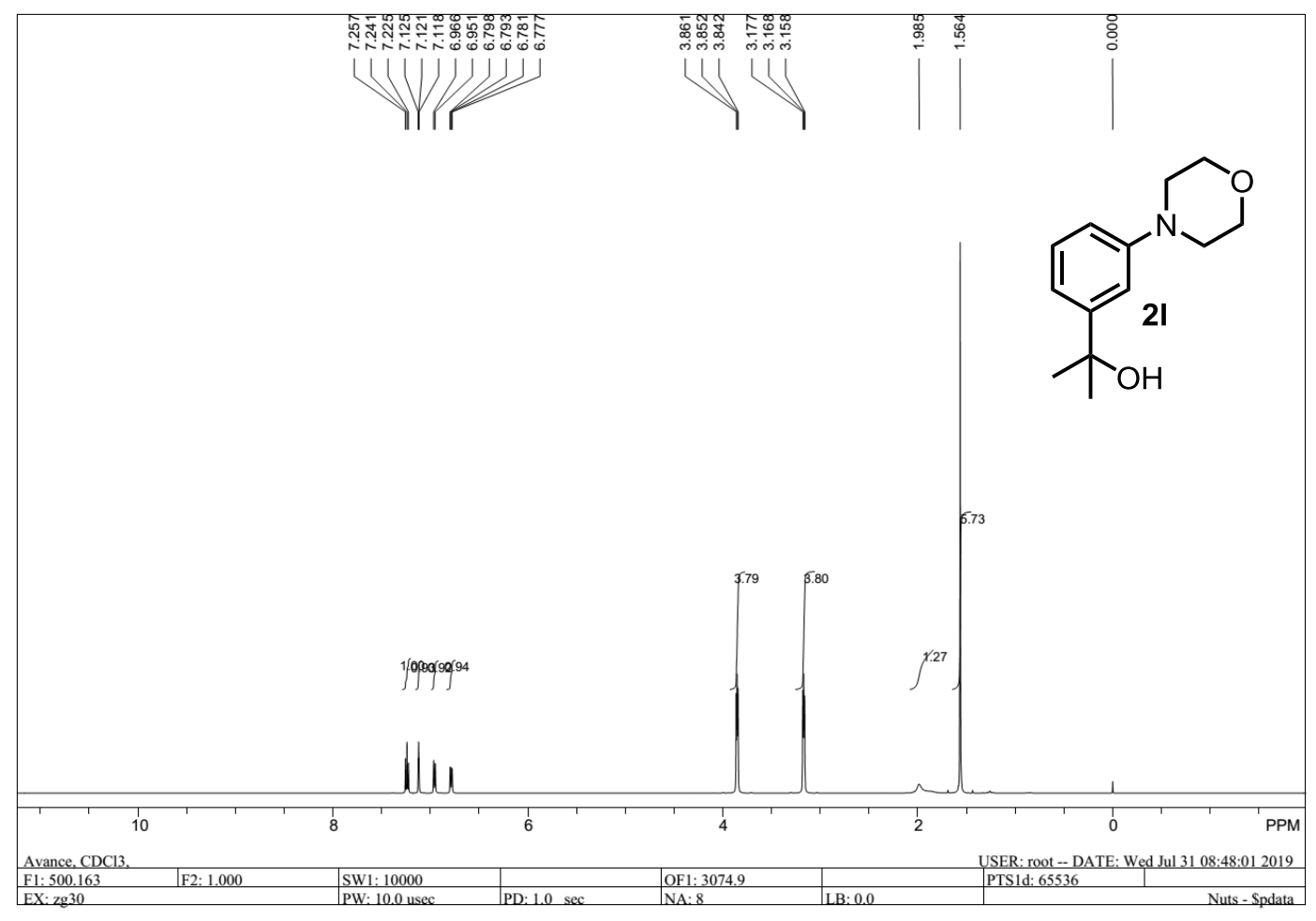

${ }^{13} \mathrm{C}$ NMR $\left(125 \mathrm{MHz}, \mathrm{CDCl}_{3}\right)$ :

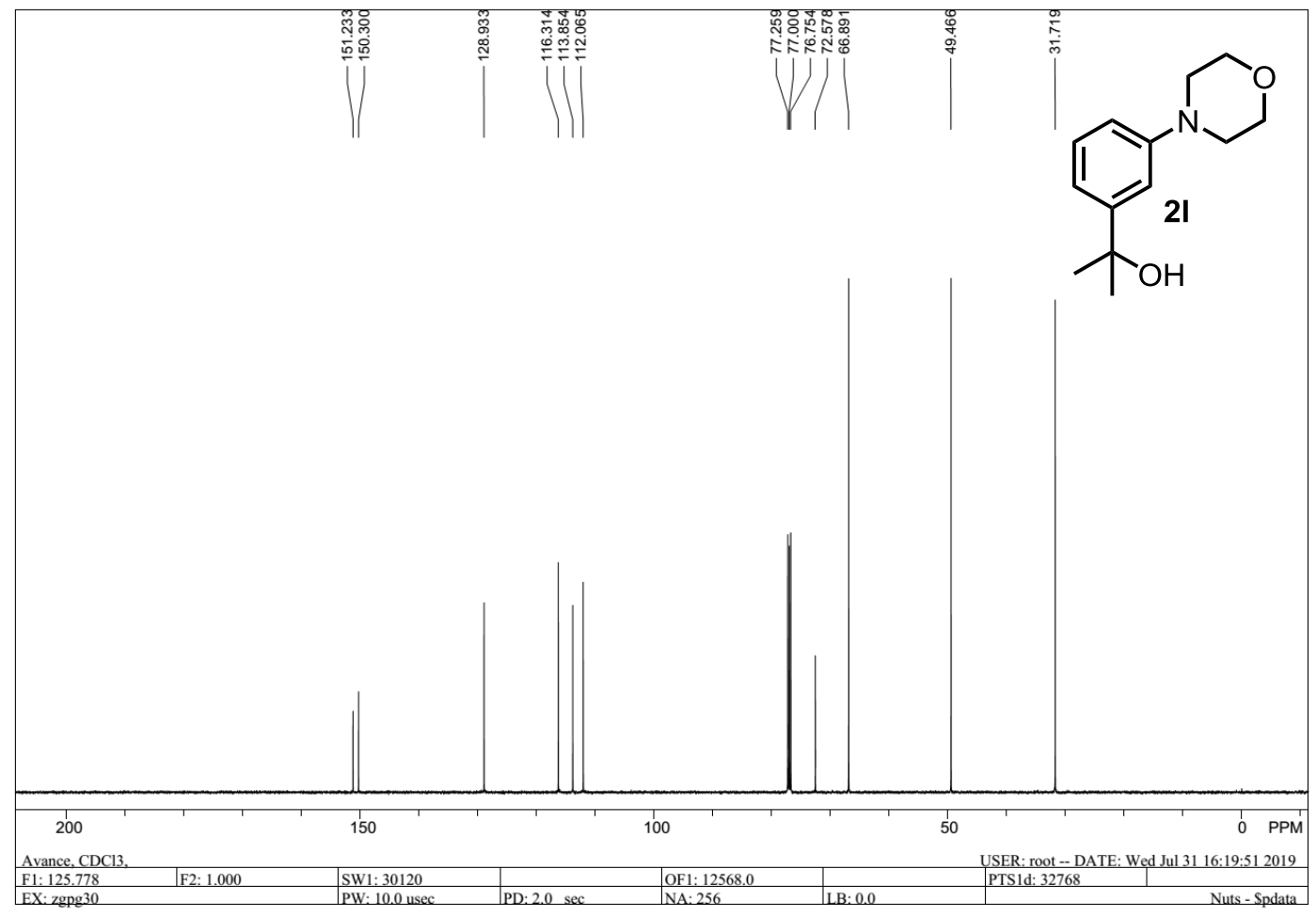


${ }^{1} \mathrm{H}$ NMR $\left(500 \mathrm{MHz}, \mathrm{CDCl}_{3}\right)$ :

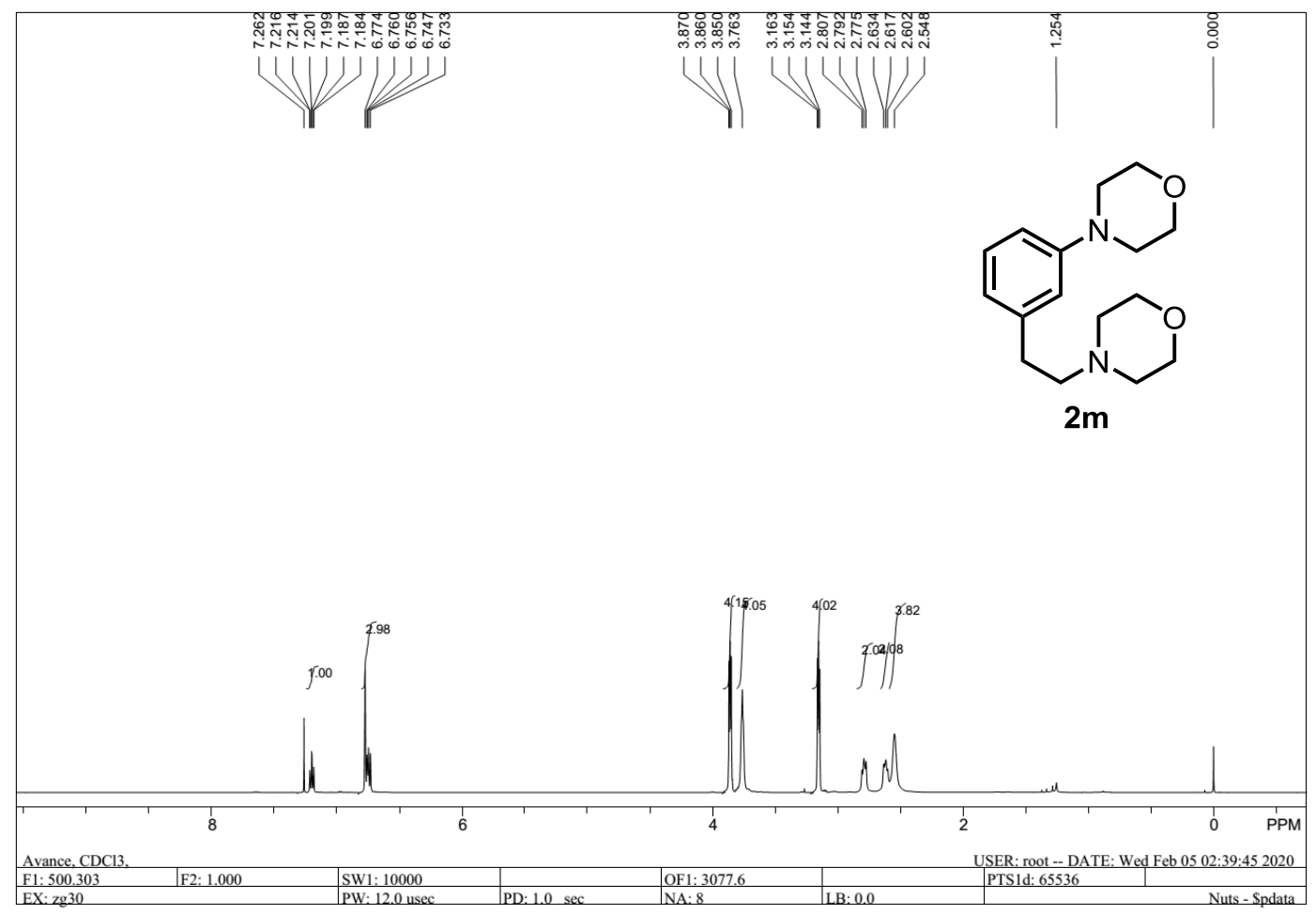

${ }^{13} \mathrm{C}$ NMR $\left(125 \mathrm{MHz}, \mathrm{CDCl}_{3}\right)$ :

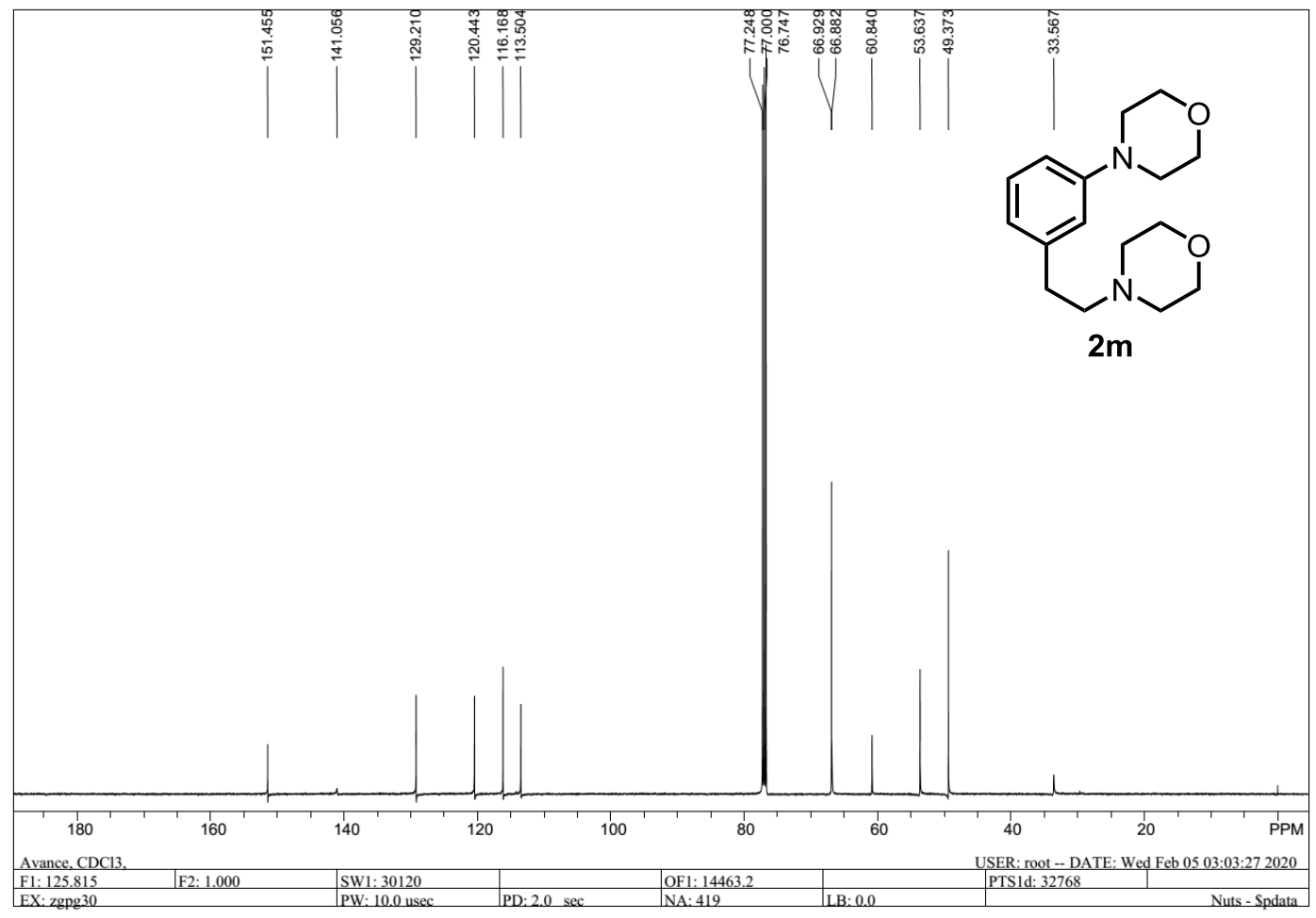


${ }^{1} \mathrm{H}$ NMR $\left(500 \mathrm{MHz}, \mathrm{CDCl}_{3}\right)$ :

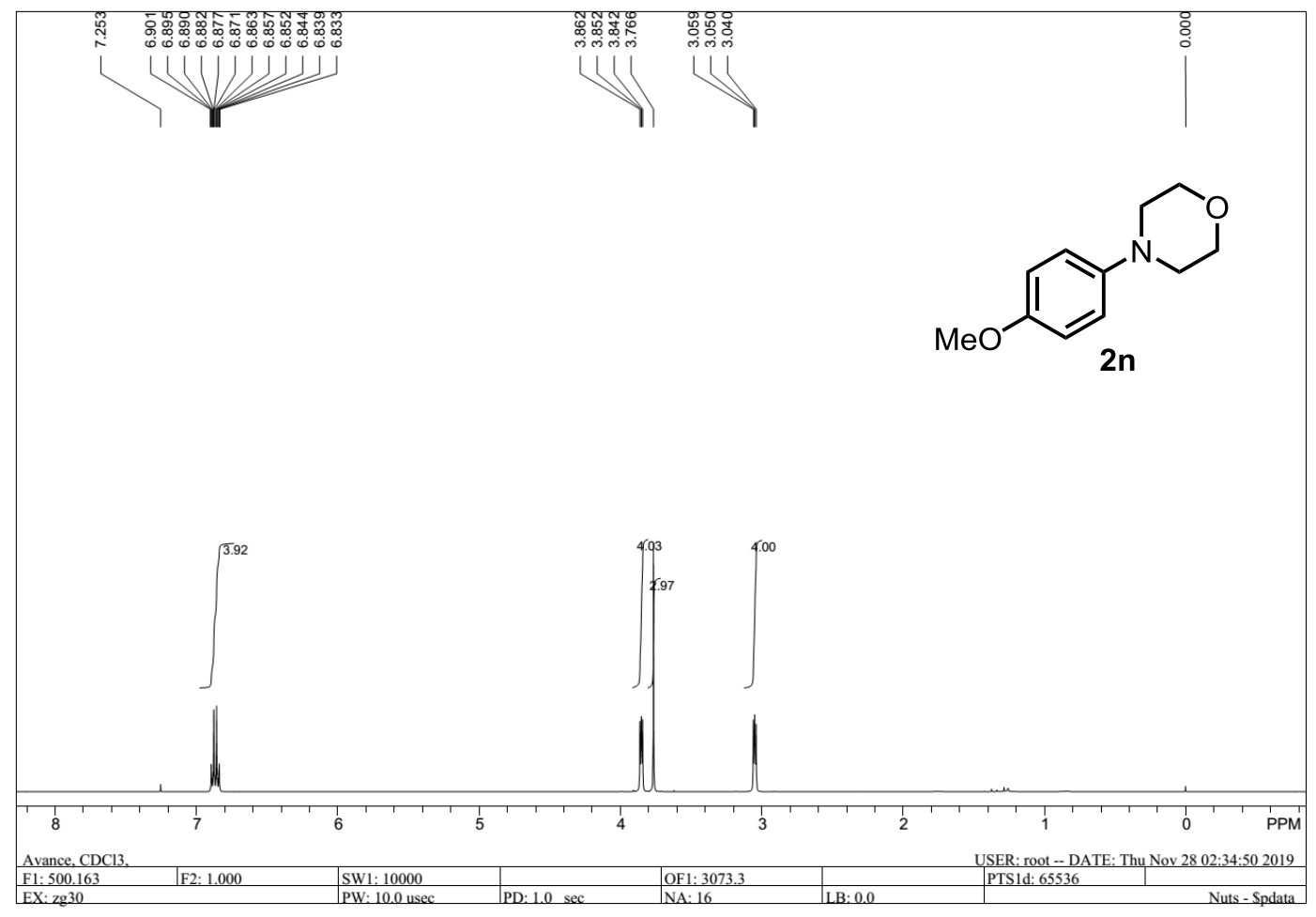

${ }^{1} \mathrm{H}$ NMR (500MHz, $\left.\mathrm{CDCl}_{3}\right)$ :

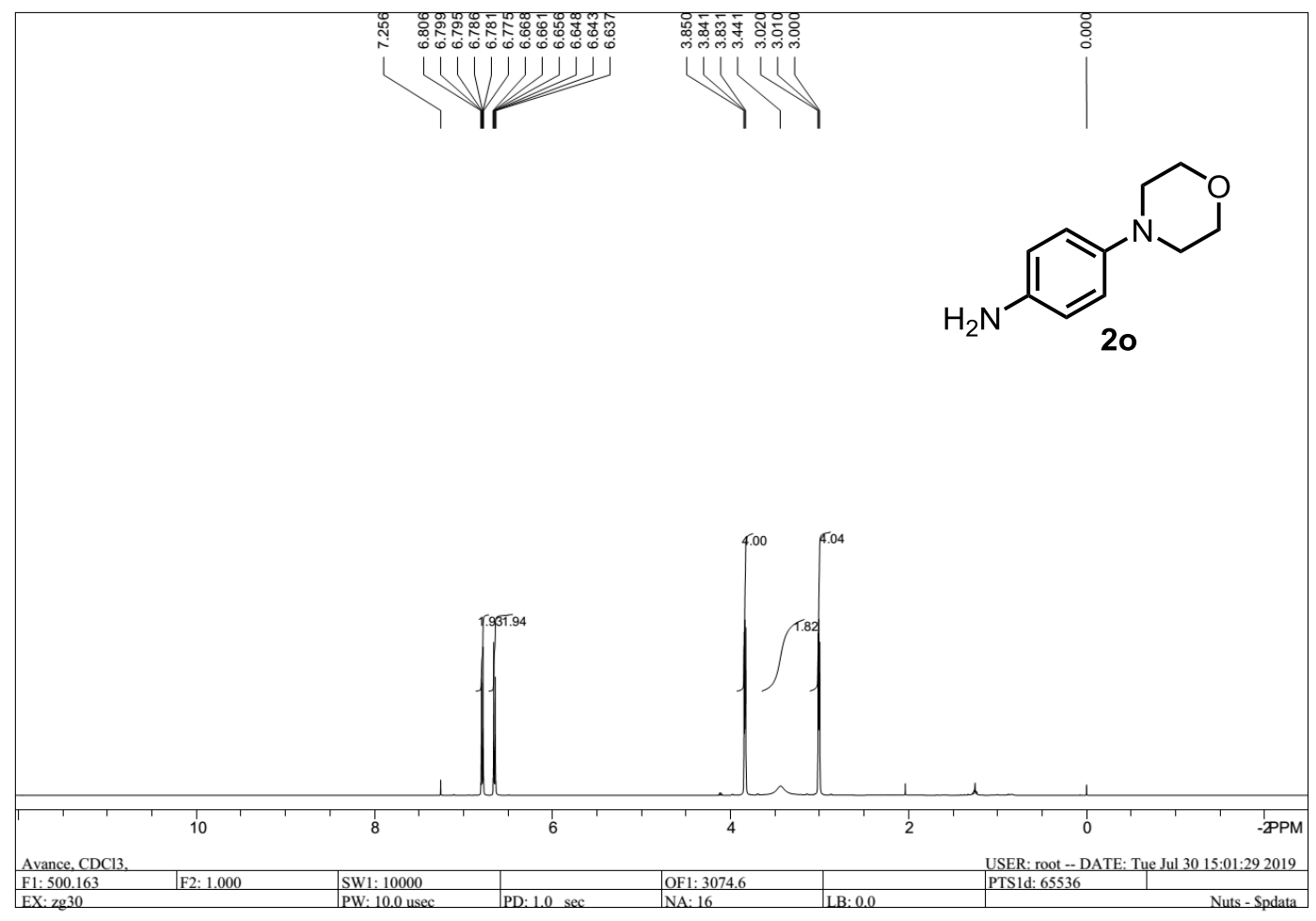


${ }^{1} \mathrm{H}$ NMR $\left(500 \mathrm{MHz}, \mathrm{CDCl}_{3}\right)$ :

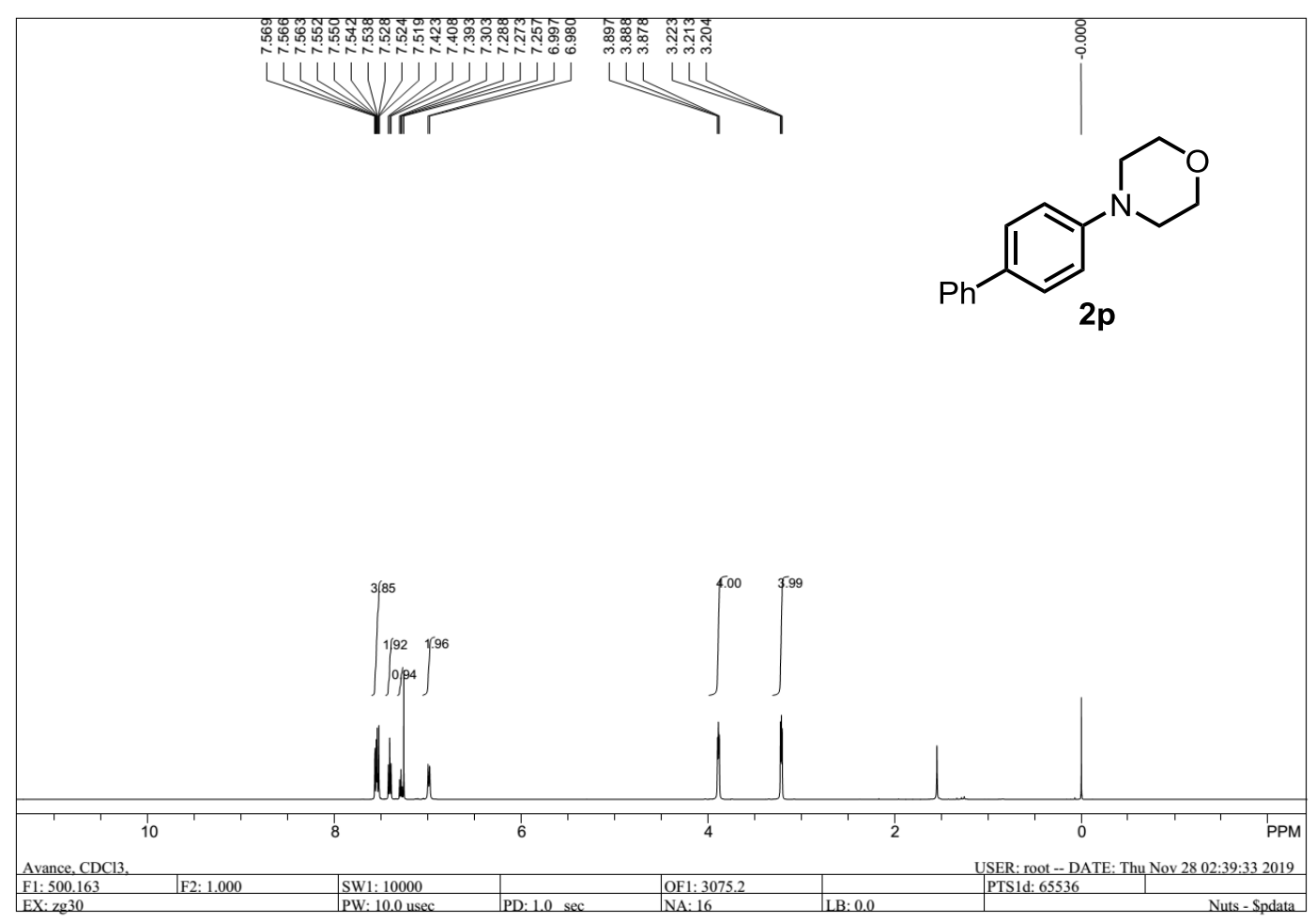


${ }^{1} \mathrm{H}$ NMR (500MHz, $\left.\mathrm{CDCl}_{3}\right)$ :

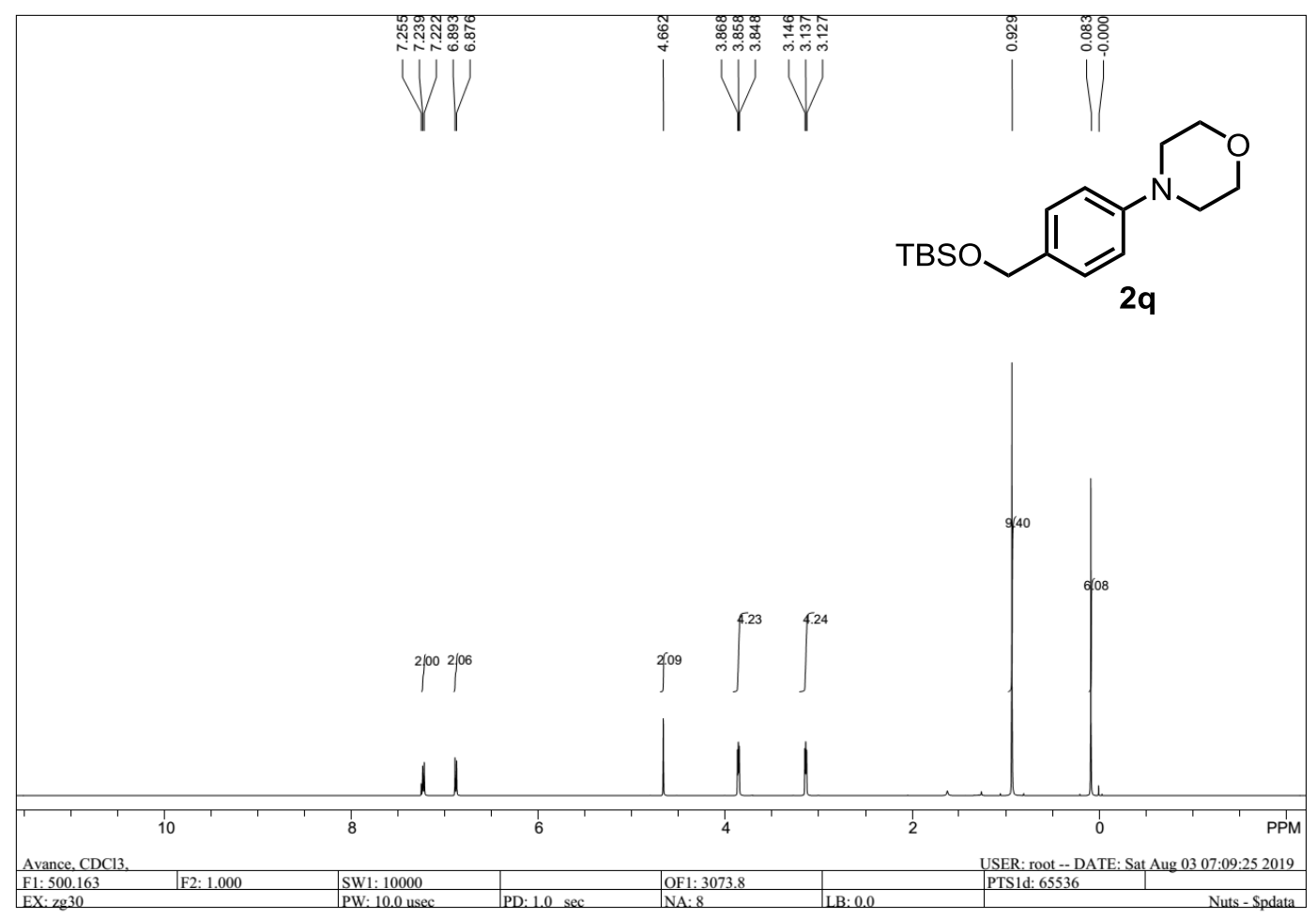

${ }^{13} \mathrm{C}$ NMR $\left(125 \mathrm{MHz}, \mathrm{CDCl}_{3}\right)$ :

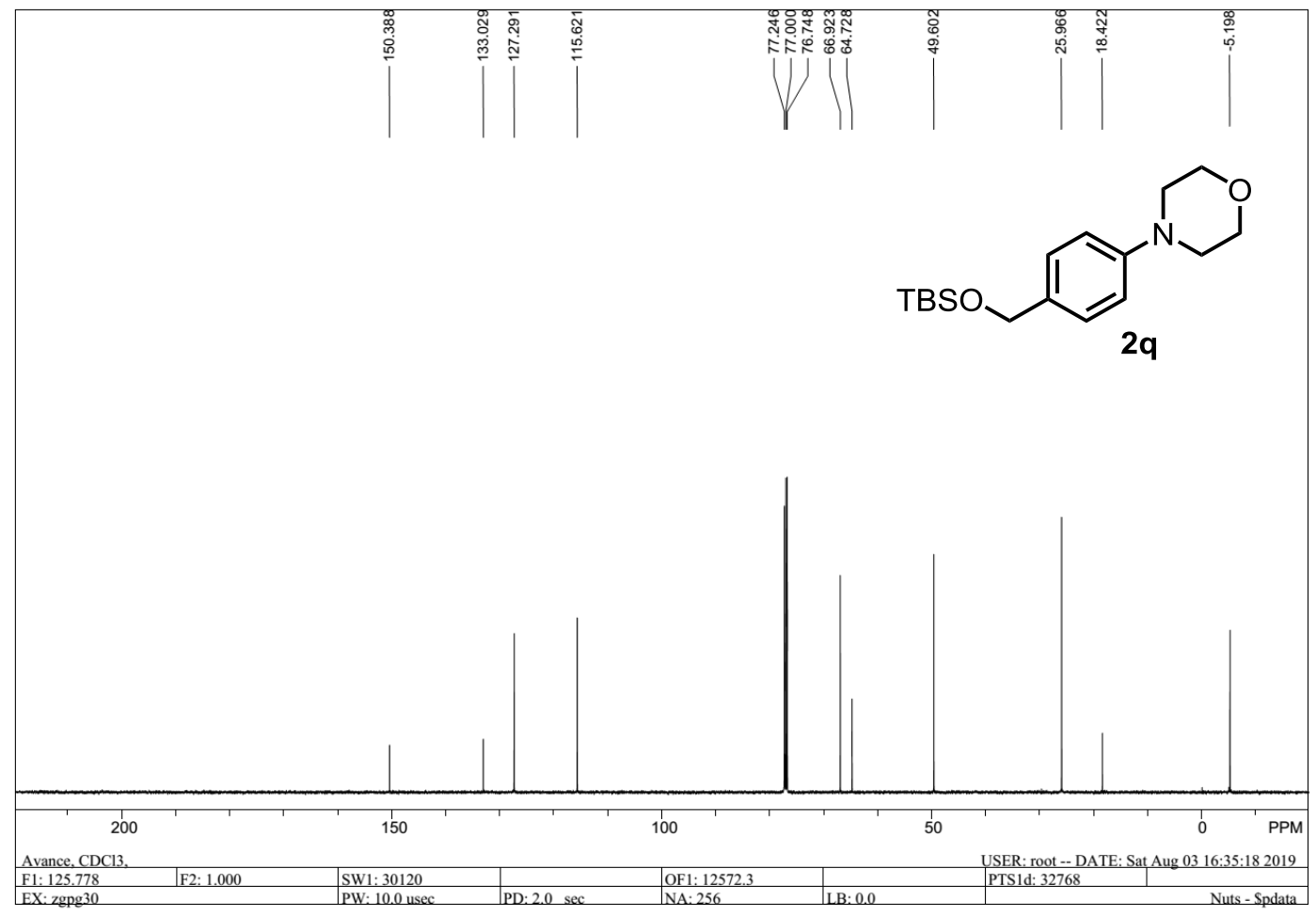


${ }^{1} \mathrm{H}$ NMR $\left(500 \mathrm{MHz}, \mathrm{CDCl}_{3}\right)$ :

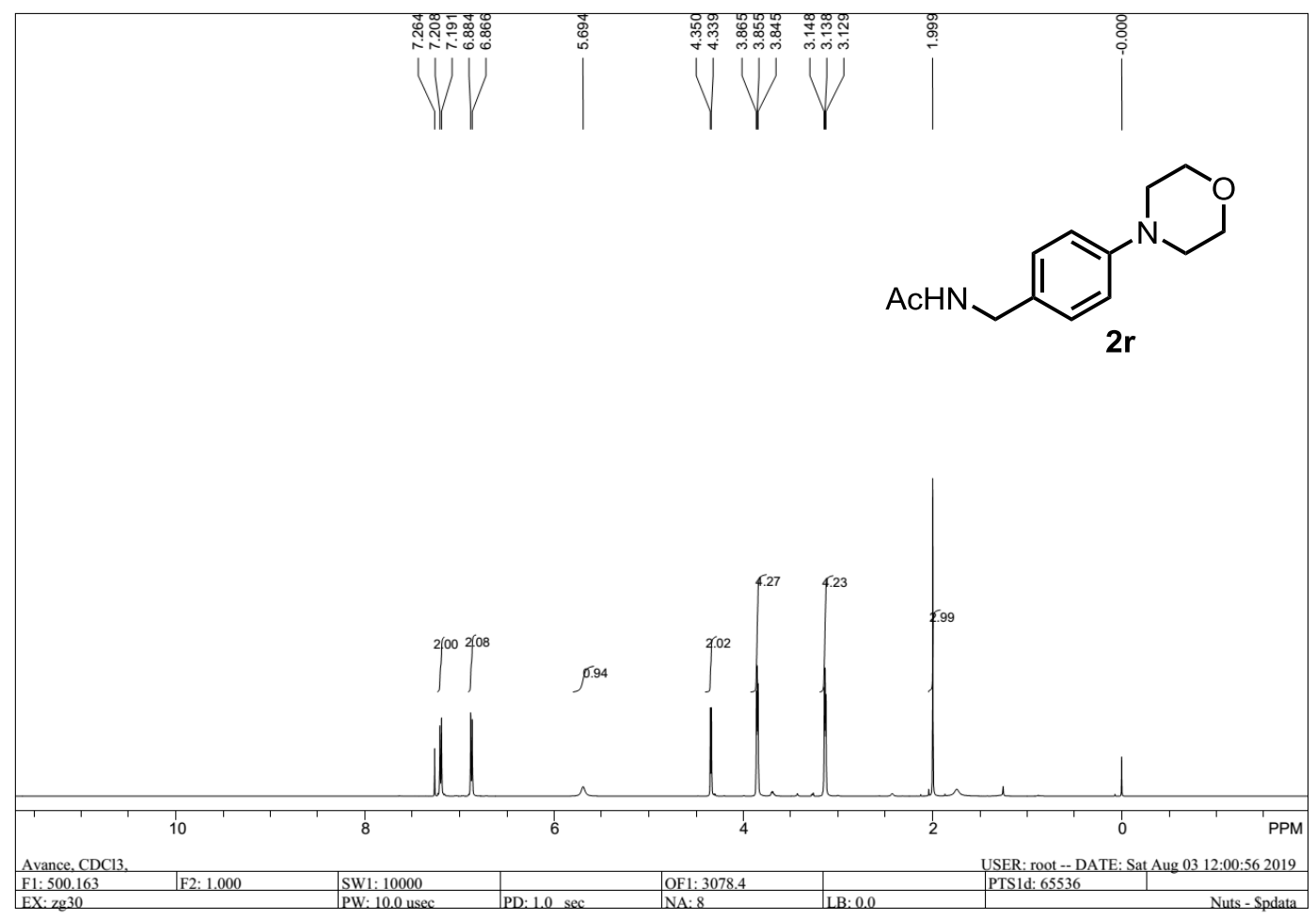

${ }^{13} \mathrm{C}$ NMR $\left(125 \mathrm{MHz}, \mathrm{CDCl}_{3}\right)$ :

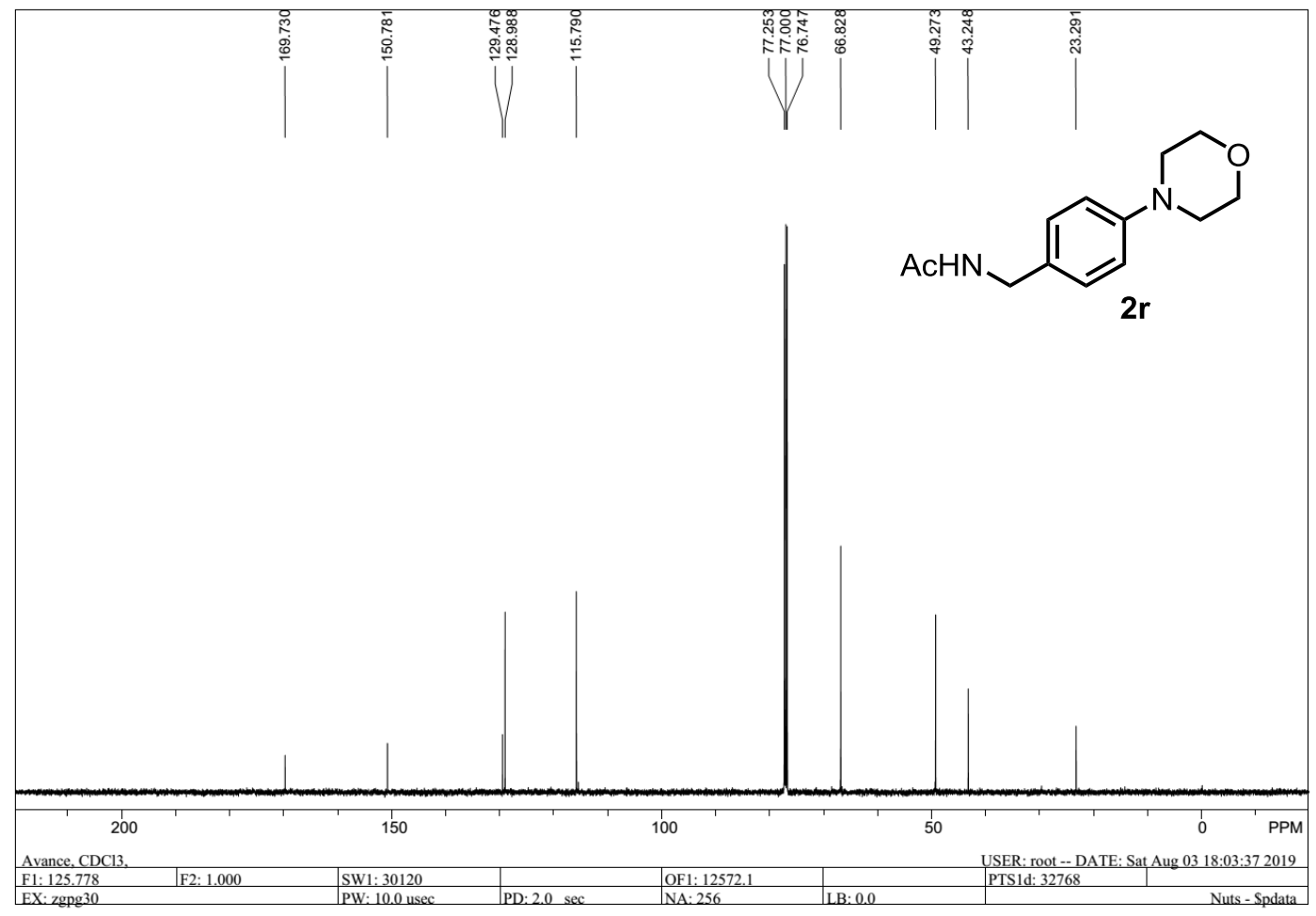


${ }^{1} \mathrm{H}$ NMR $\left(500 \mathrm{MHz}, \mathrm{CDCl}_{3}\right)$ :

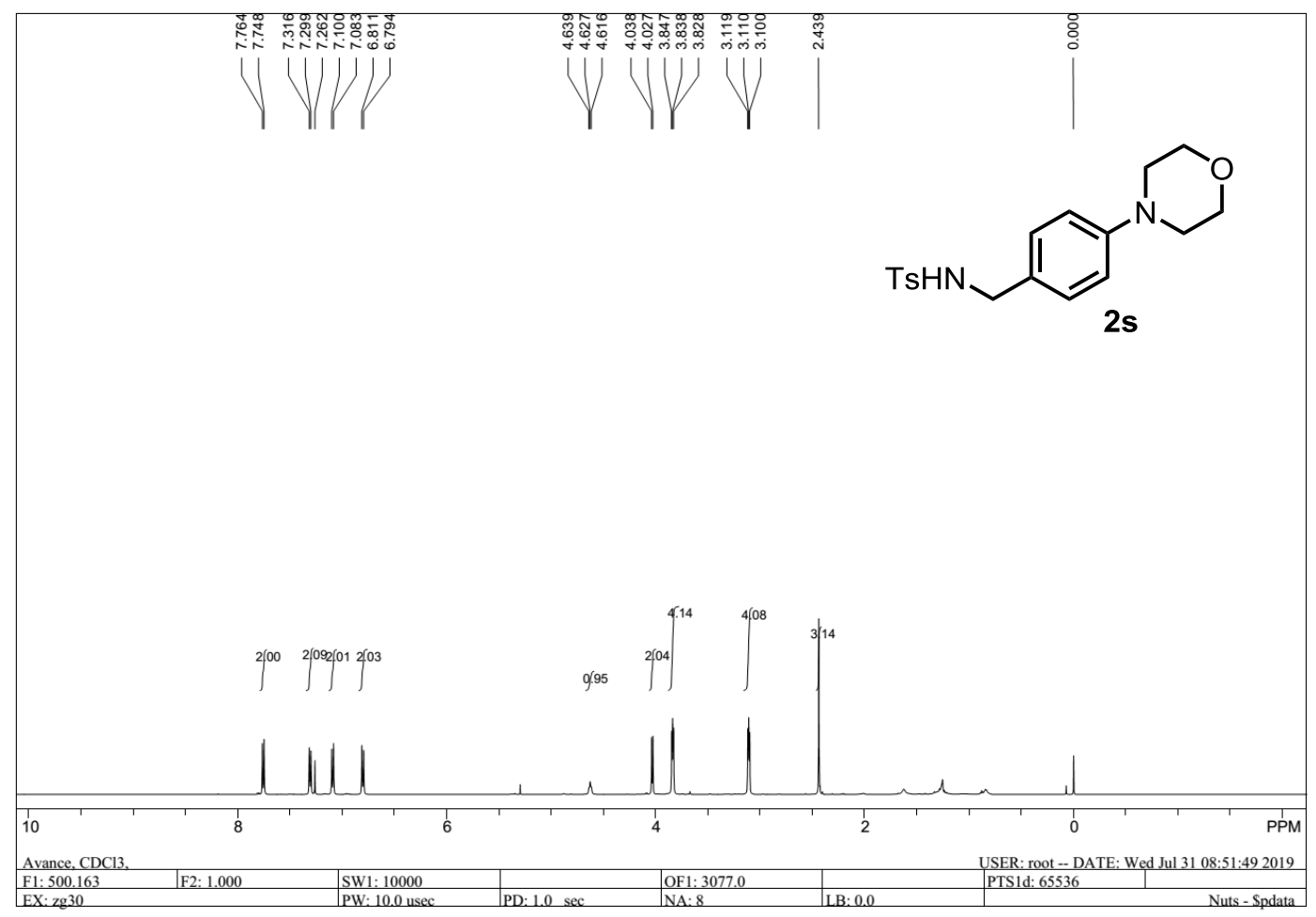

${ }^{13} \mathrm{C}$ NMR $\left(125 \mathrm{MHz}, \mathrm{CDCl}_{3}\right)$ :

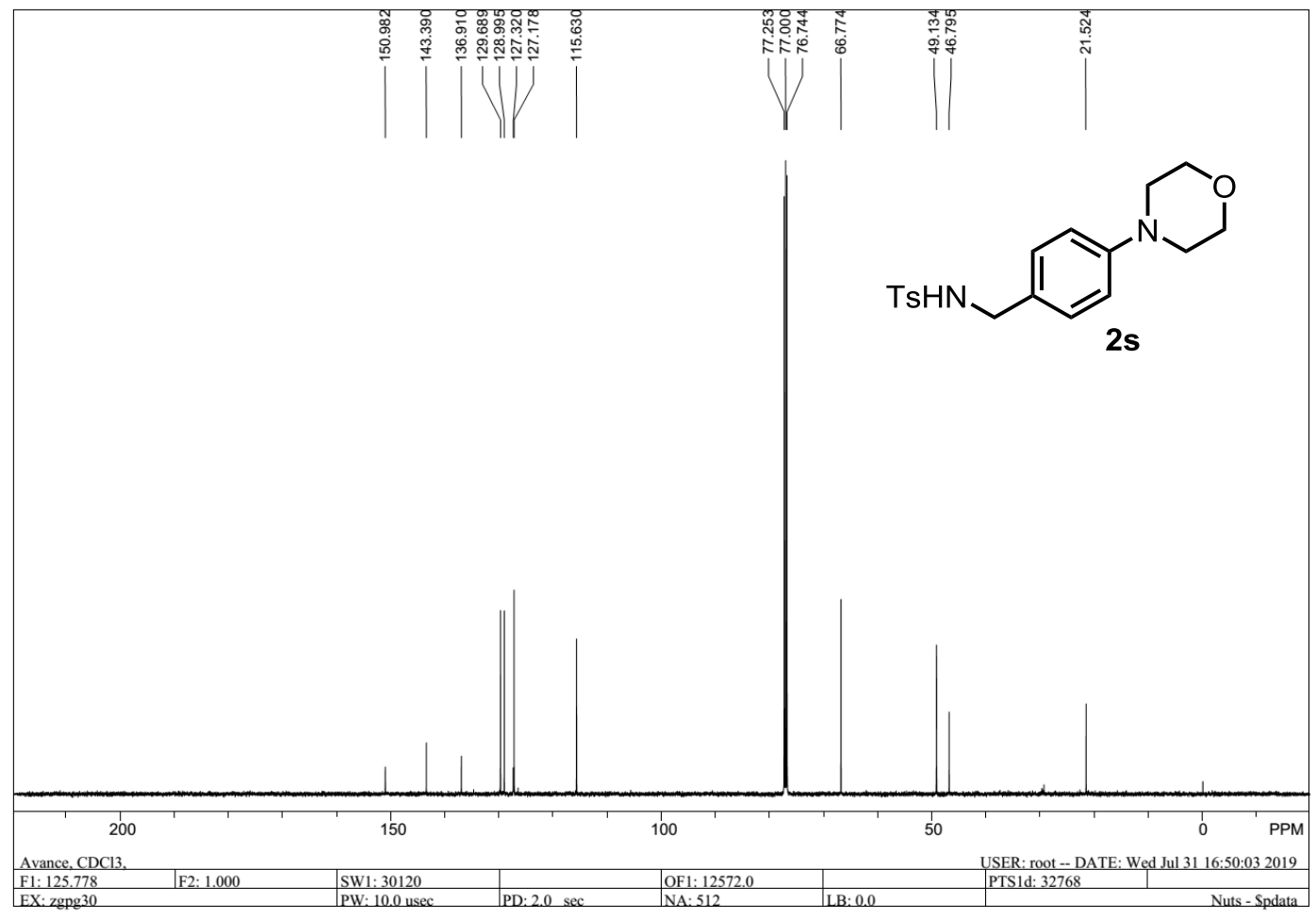


${ }^{1} \mathrm{H}$ NMR (500MHz, $\left.\mathrm{CDCl}_{3}\right)$ :

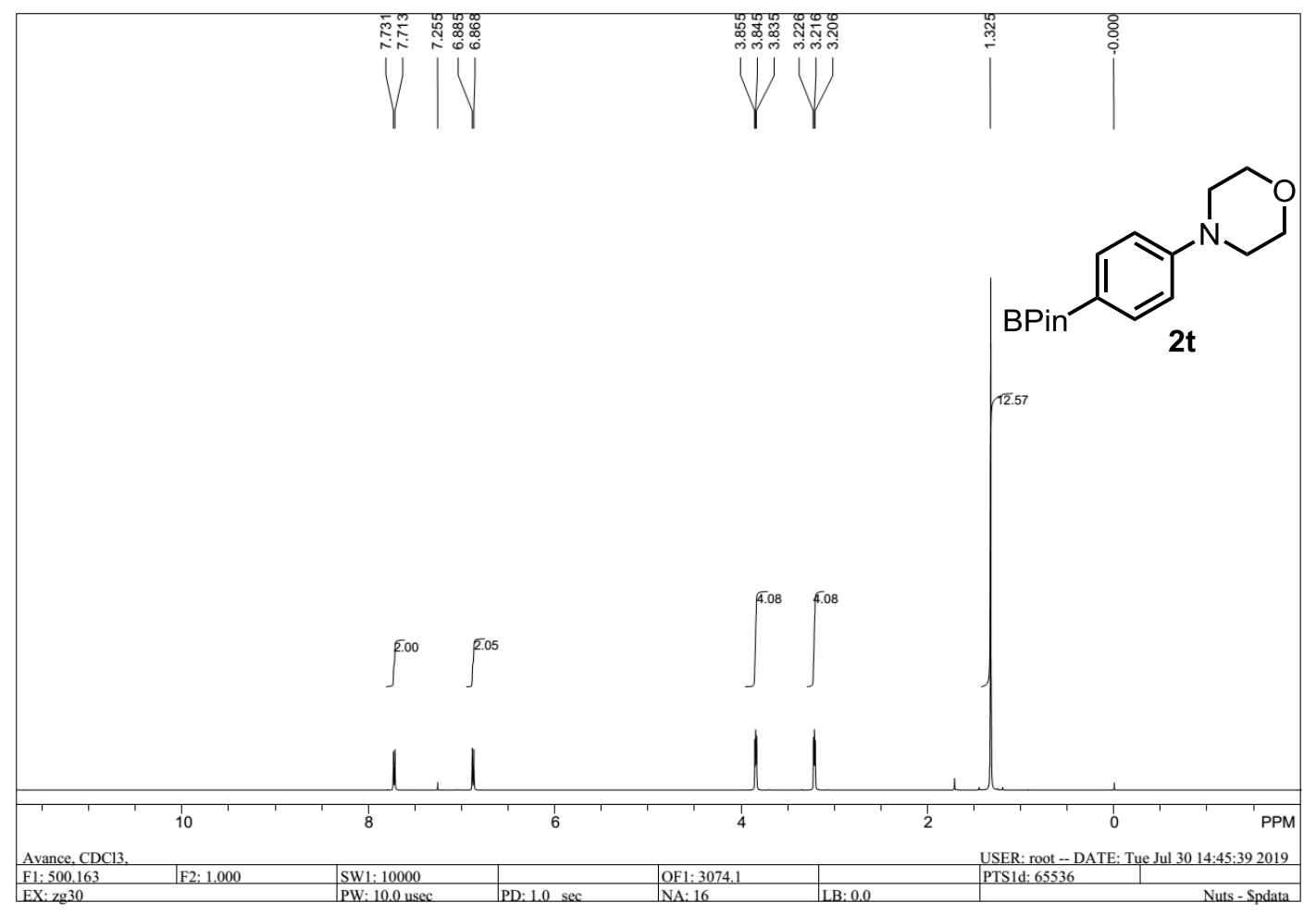

${ }^{1} \mathrm{H} \mathrm{NMR}\left(500 \mathrm{MHz}, \mathrm{CDCl}_{3}\right)$ :

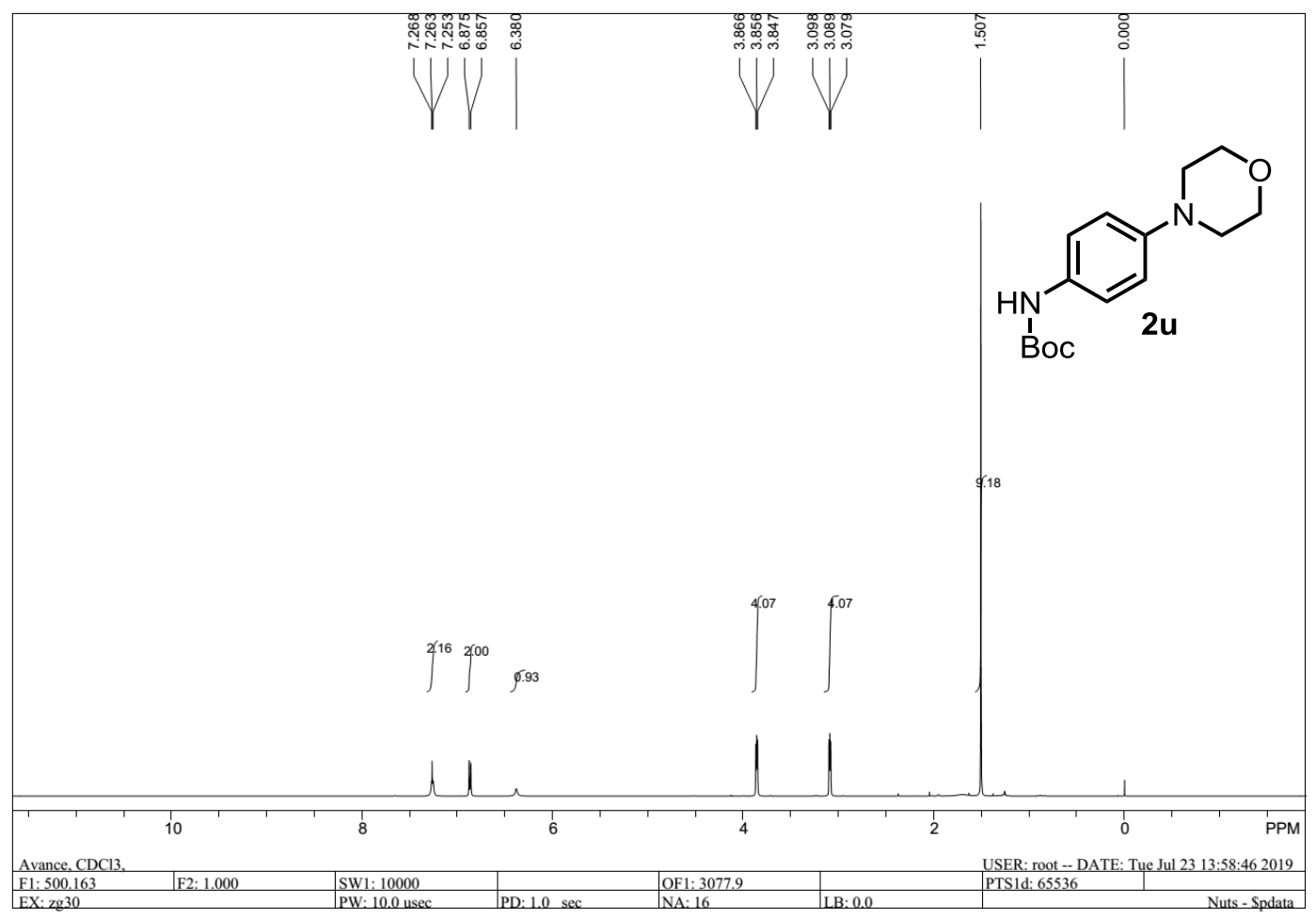


${ }^{1} \mathrm{H}$ NMR $\left(500 \mathrm{MHz}, \mathrm{CDCl}_{3}\right)$ :

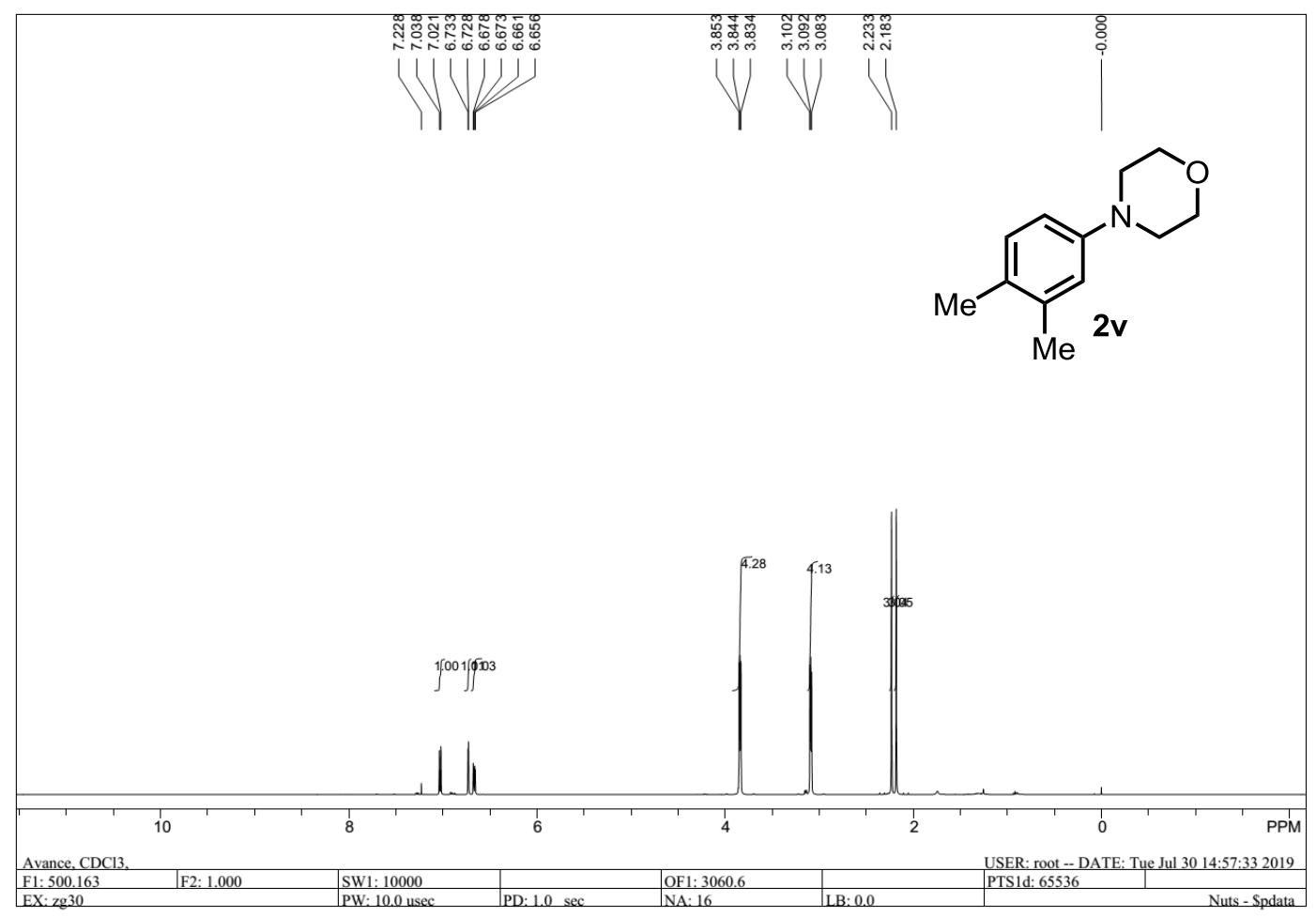


${ }^{1} \mathrm{H}$ NMR (500MHz, $\mathrm{CDCl}_{3}$ ):

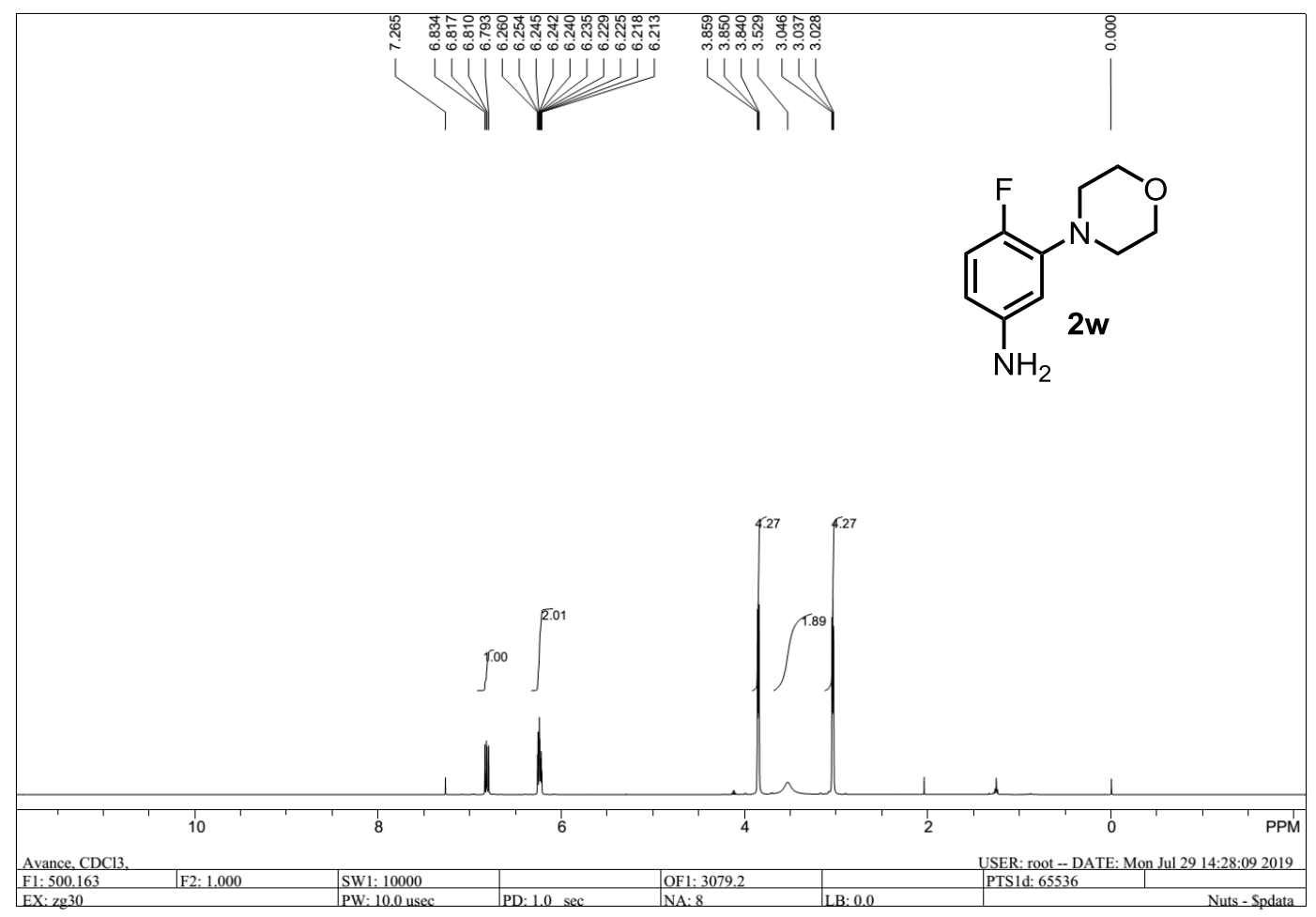

${ }^{13} \mathrm{C}$ NMR $\left(125 \mathrm{MHz}, \mathrm{CDCl}_{3}\right)$ :

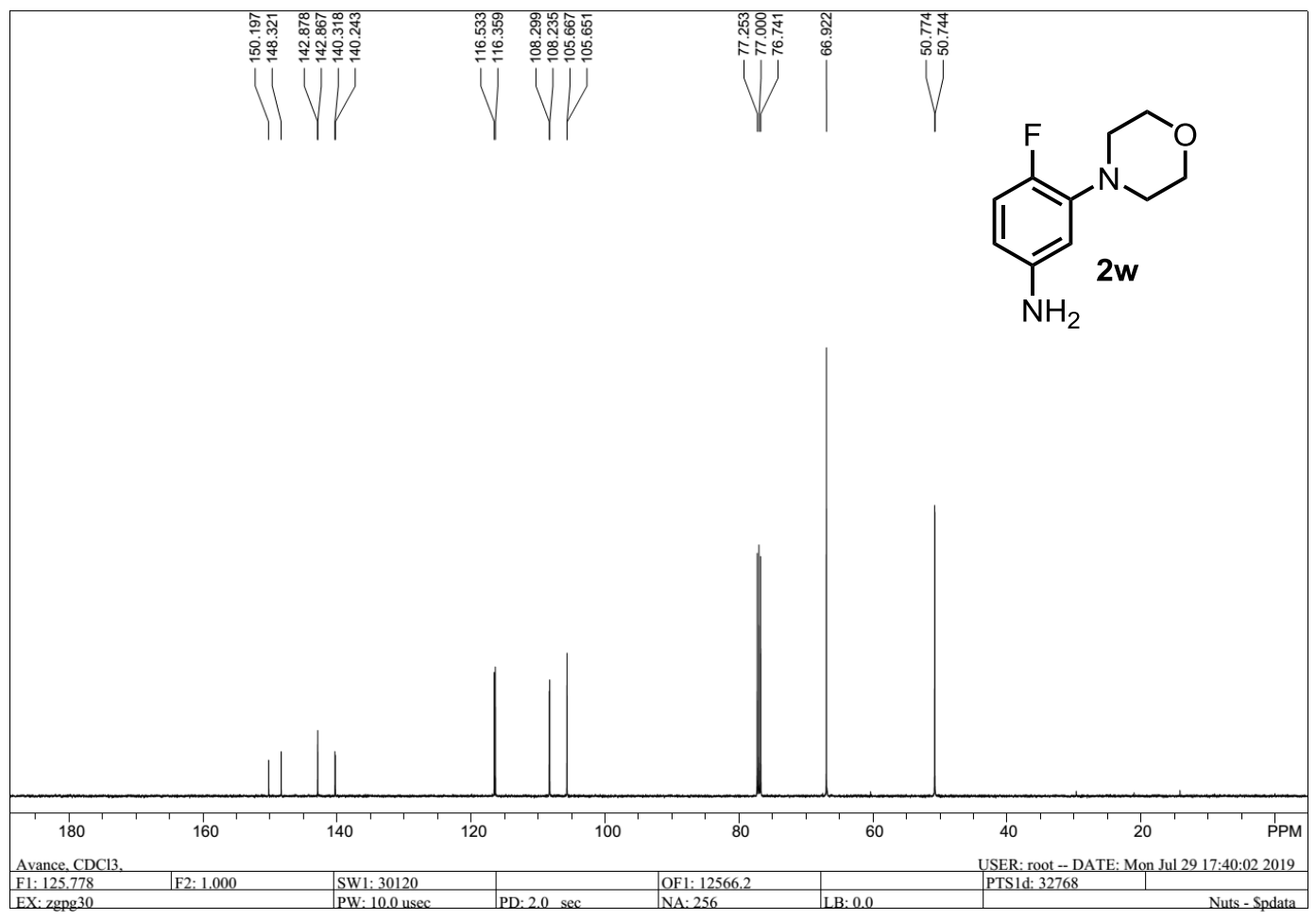


${ }^{19} \mathrm{~F}$ NMR (470MHz, $\left.\mathrm{CDCl}_{3}\right)$ :

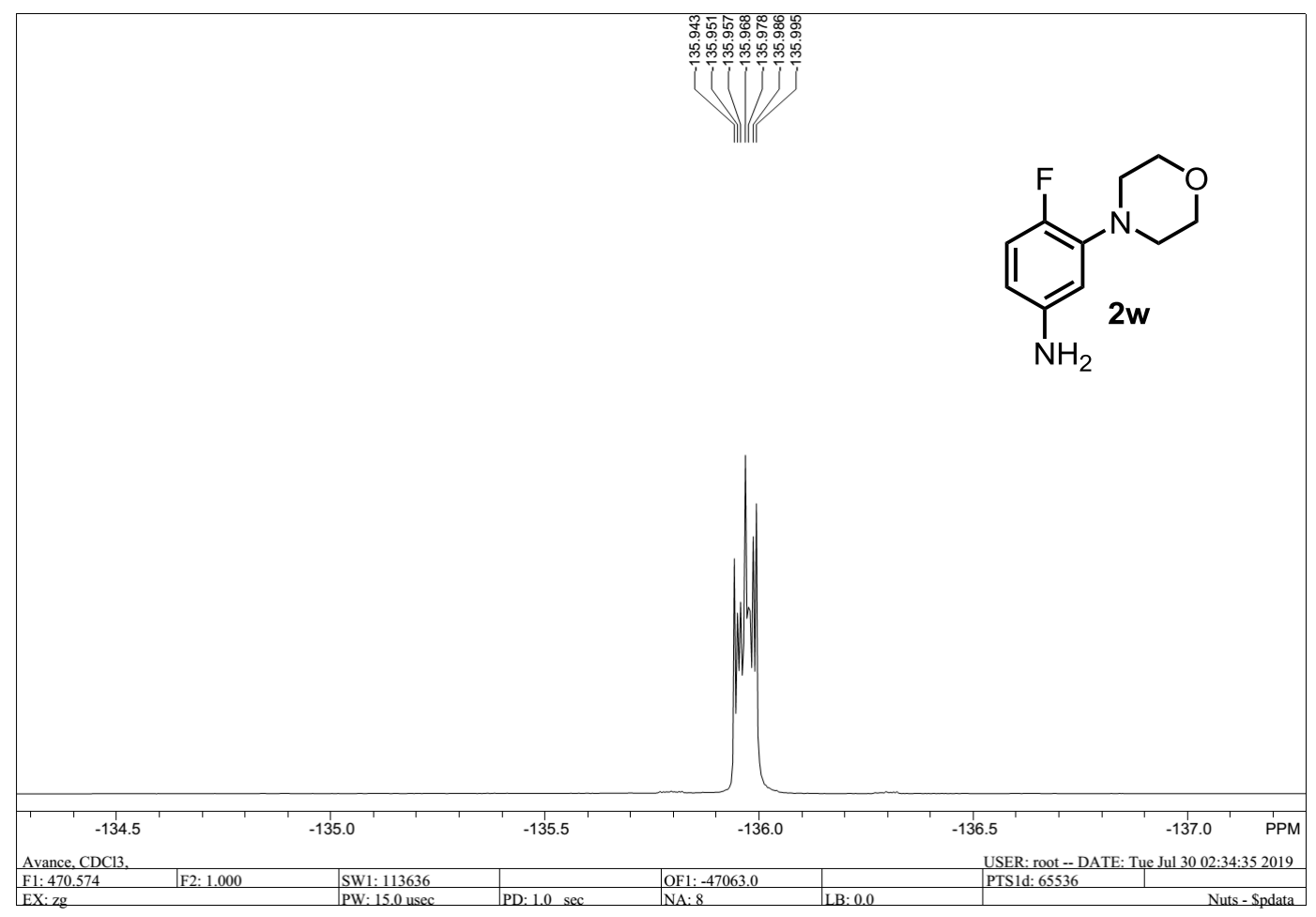


${ }^{1} \mathrm{H}$ NMR $\left(500 \mathrm{MHz}, \mathrm{CDCl}_{3}\right)$ :

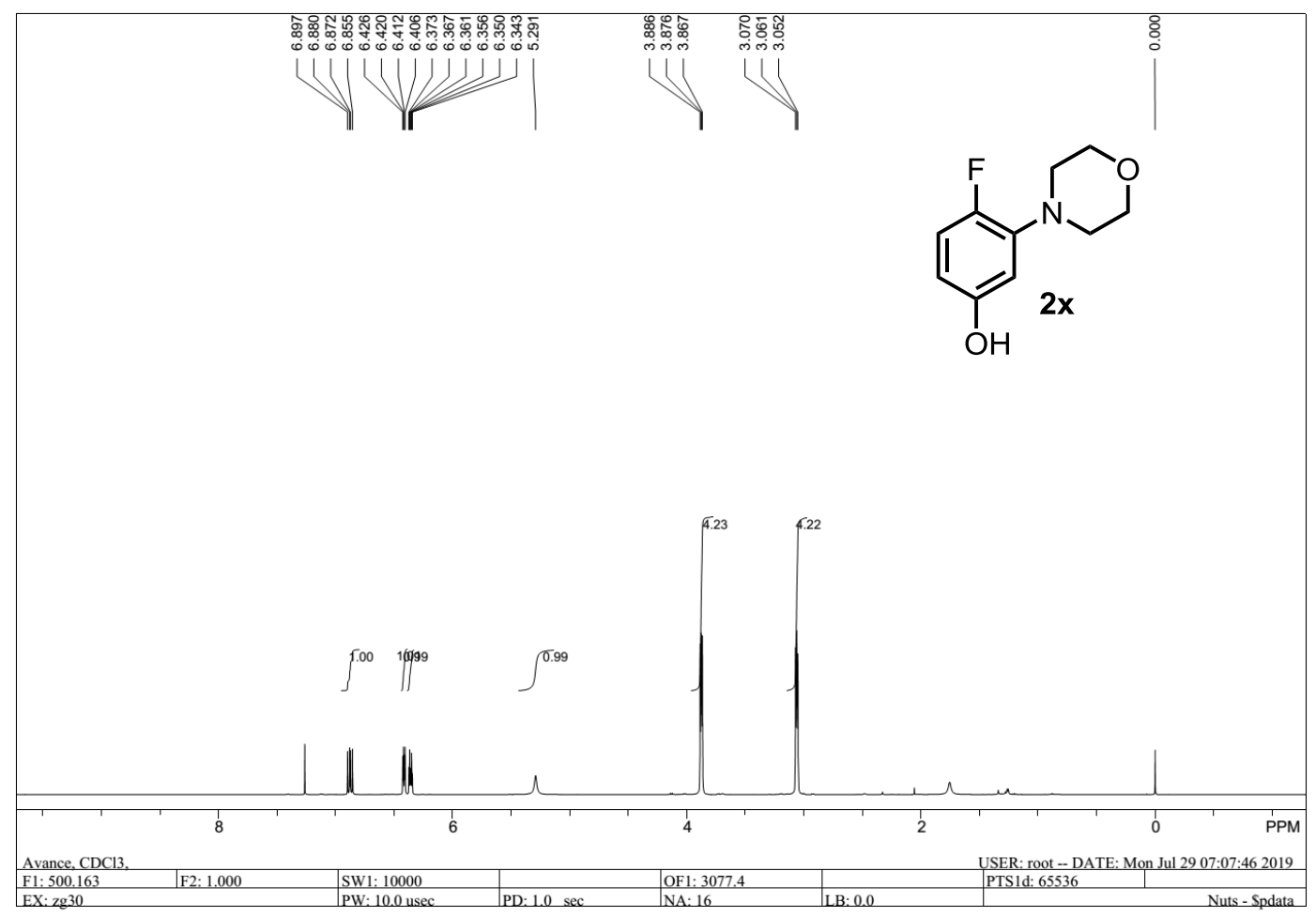

${ }^{13} \mathrm{C}$ NMR $\left(125 \mathrm{MHz}, \mathrm{CDCl}_{3}\right)$ :

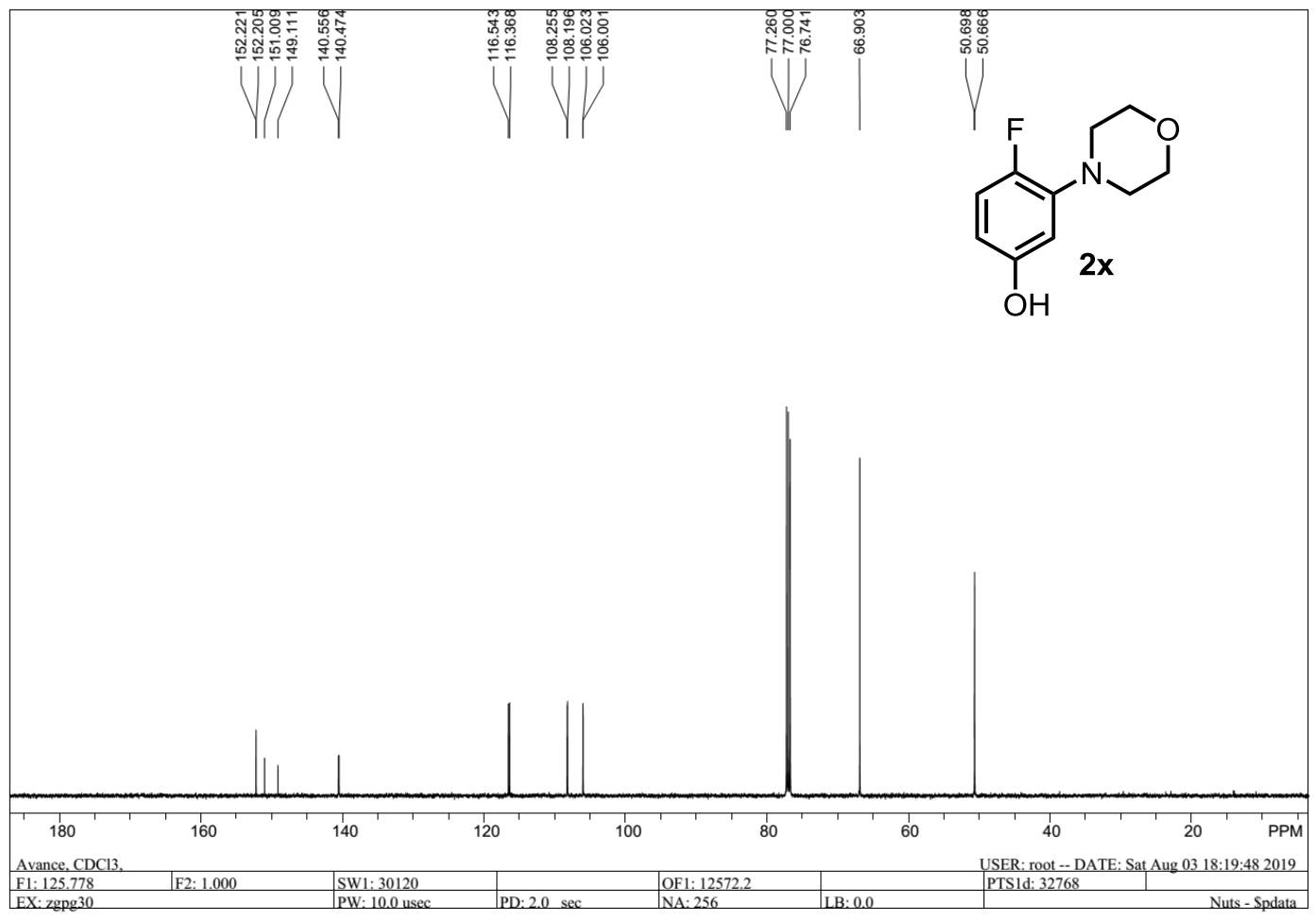


${ }^{19} \mathrm{~F}$ NMR (470MHz, $\left.\mathrm{CDCl}_{3}\right)$ :

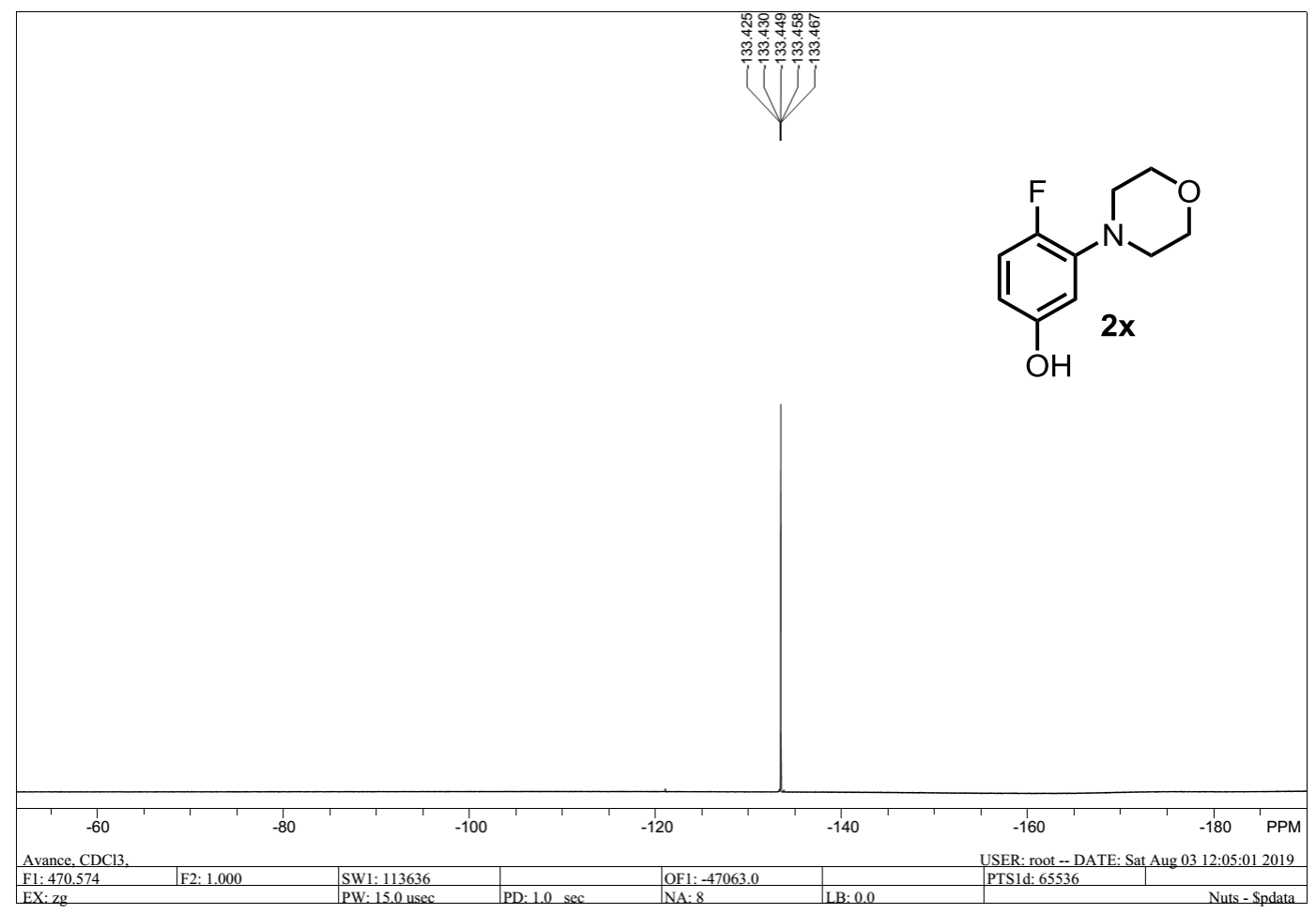


${ }^{1} \mathrm{H}$ NMR $\left(500 \mathrm{MHz}, \mathrm{CDCl}_{3}\right)$ :

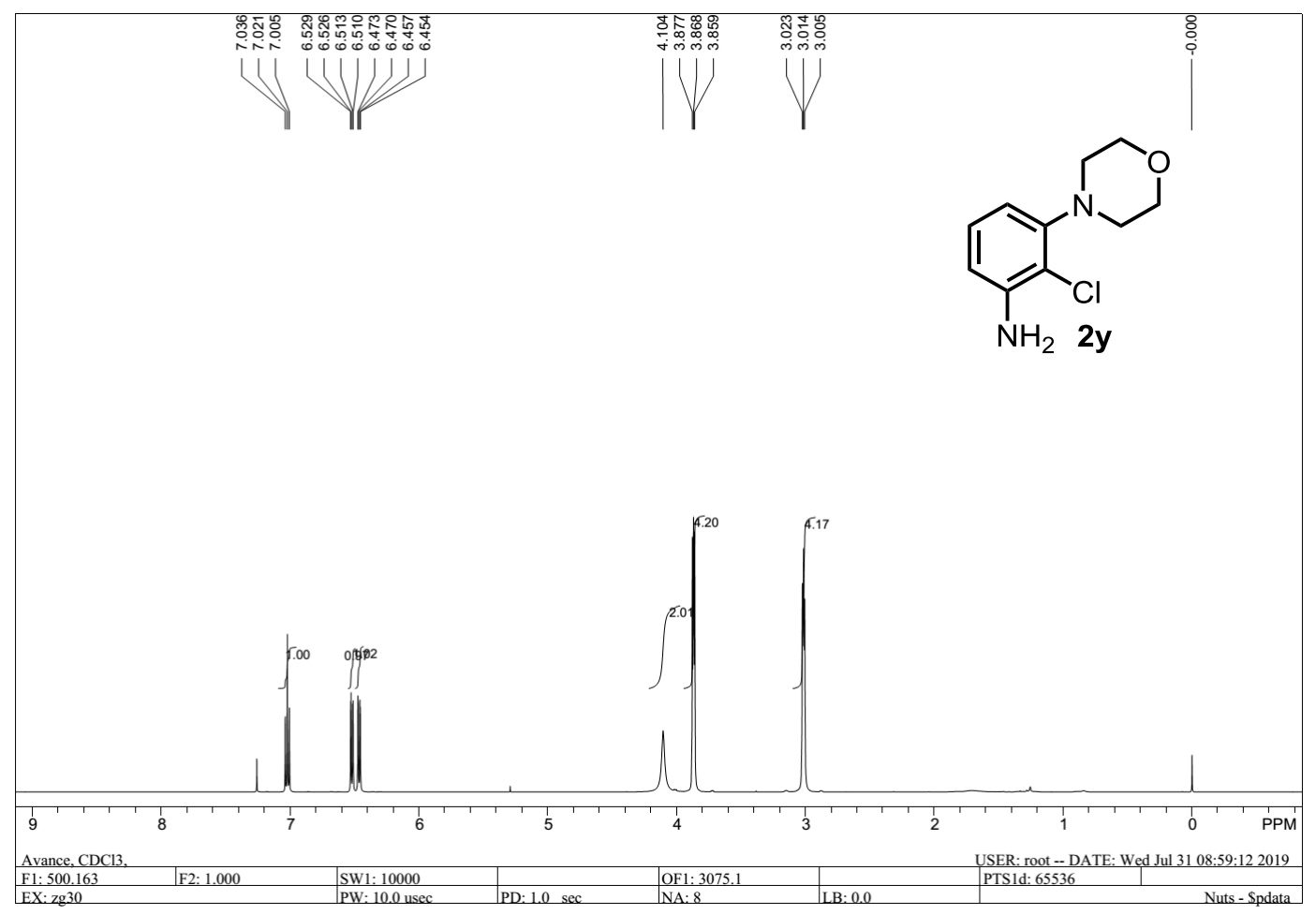

${ }^{13} \mathrm{C}$ NMR $\left(125 \mathrm{MHz}, \mathrm{CDCl}_{3}\right)$ :

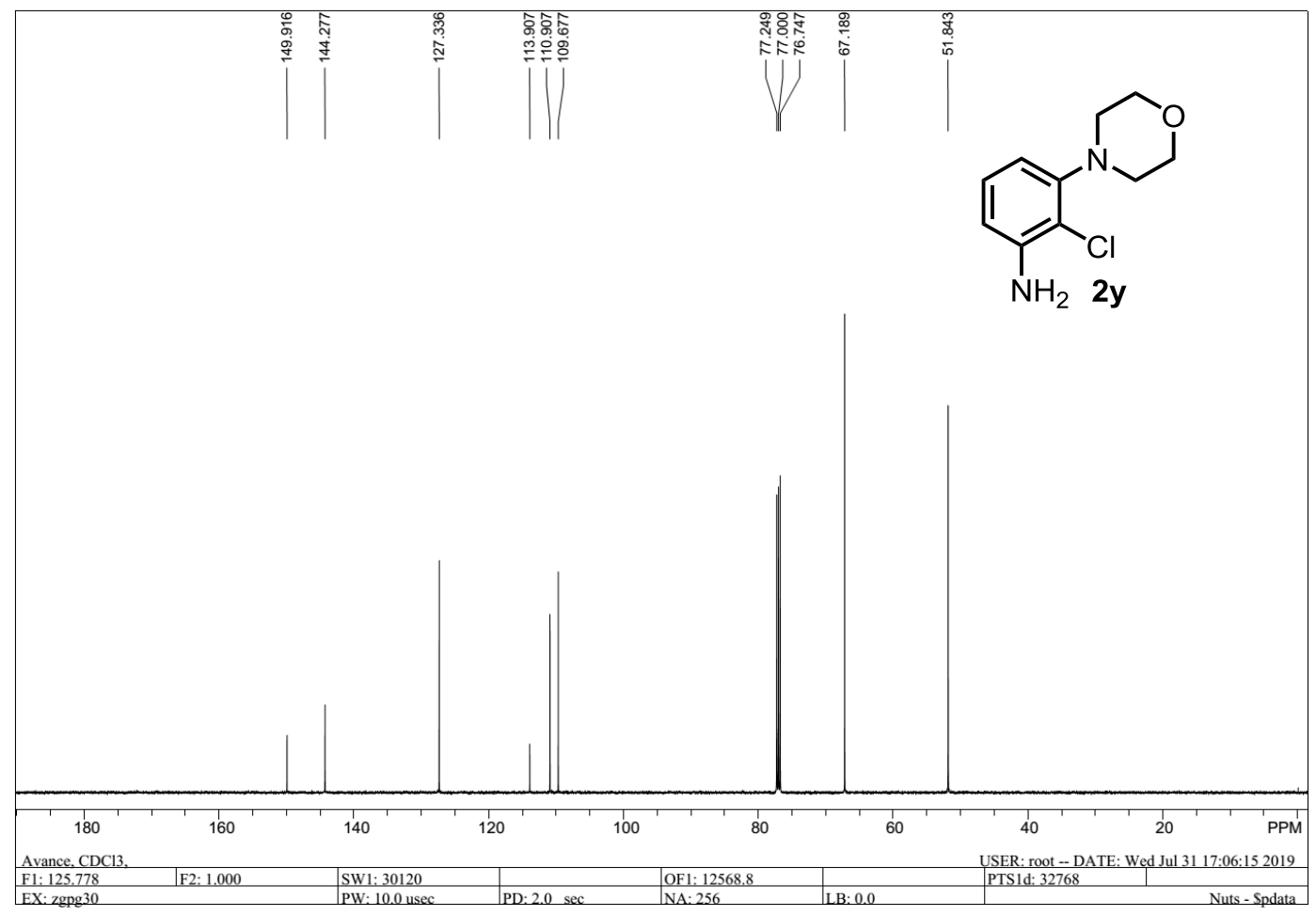


${ }^{1} \mathrm{H}$ NMR (600MHz, $\left.\mathrm{CDCl}_{3}\right)$ :

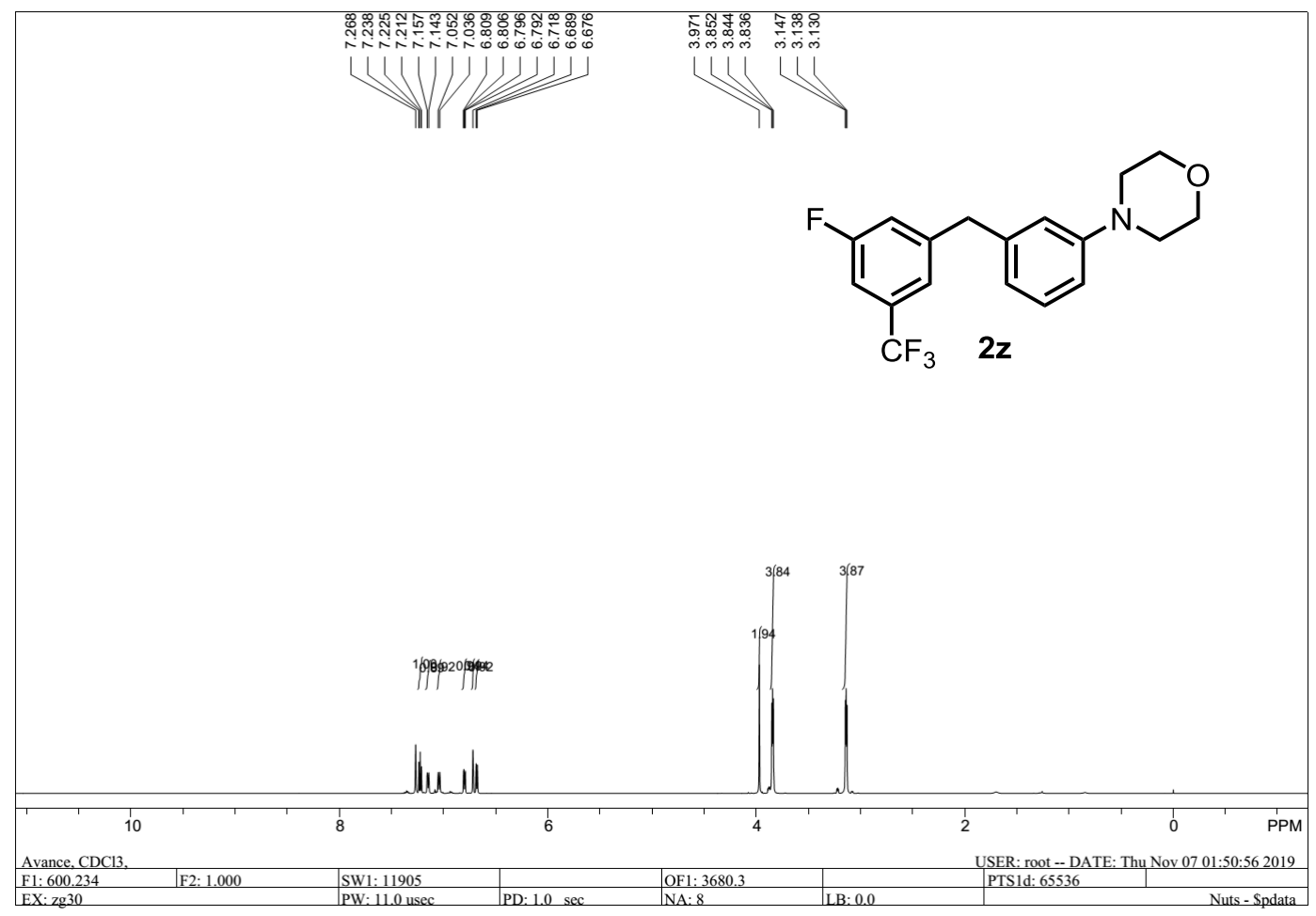

${ }^{13} \mathrm{C}$ NMR $\left(150 \mathrm{MHz}, \mathrm{CDCl}_{3}\right)$ :

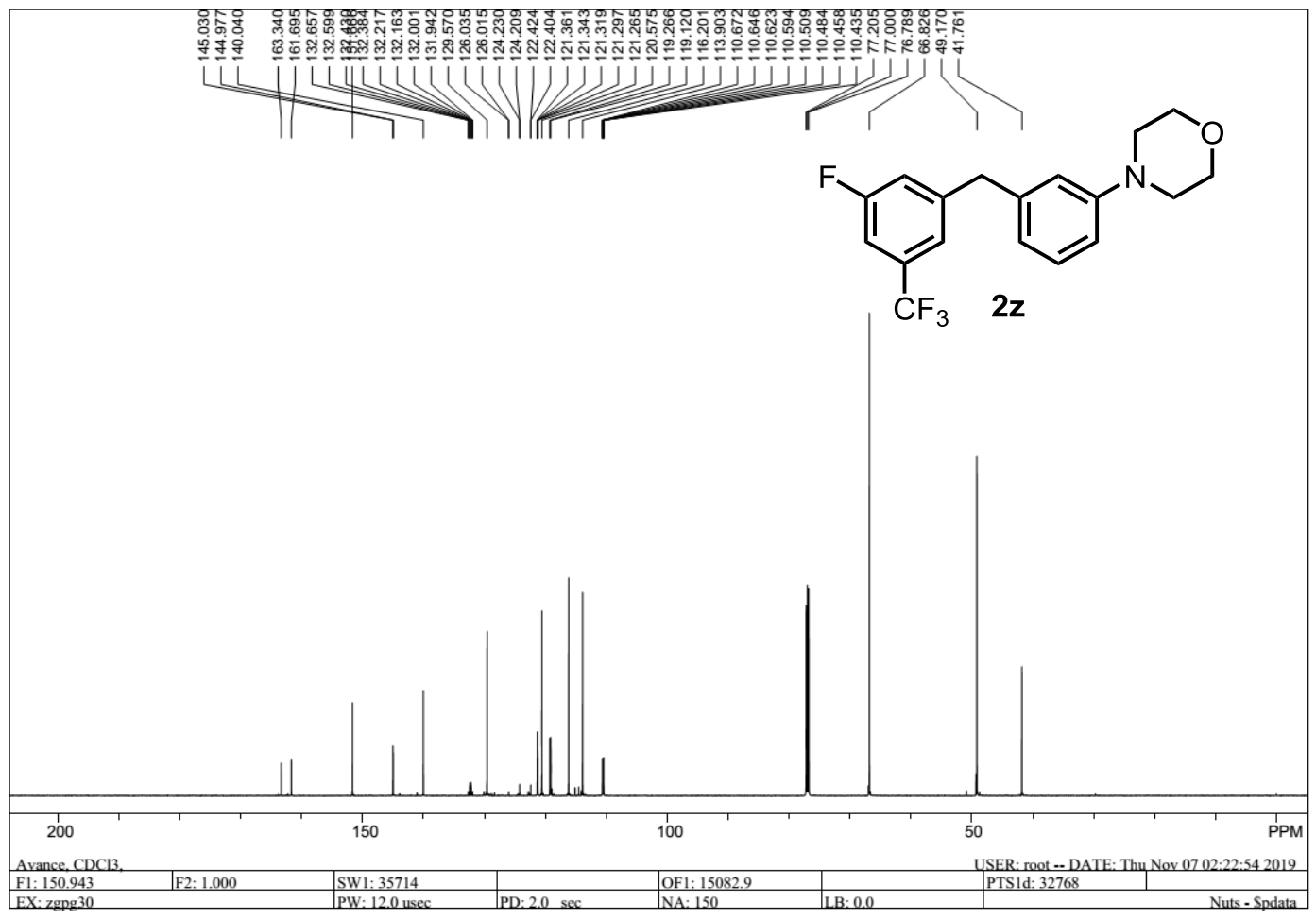


${ }^{19} \mathrm{~F} \mathrm{NMR}$ (470MHz, $\mathrm{CDCl}_{3}$ ):

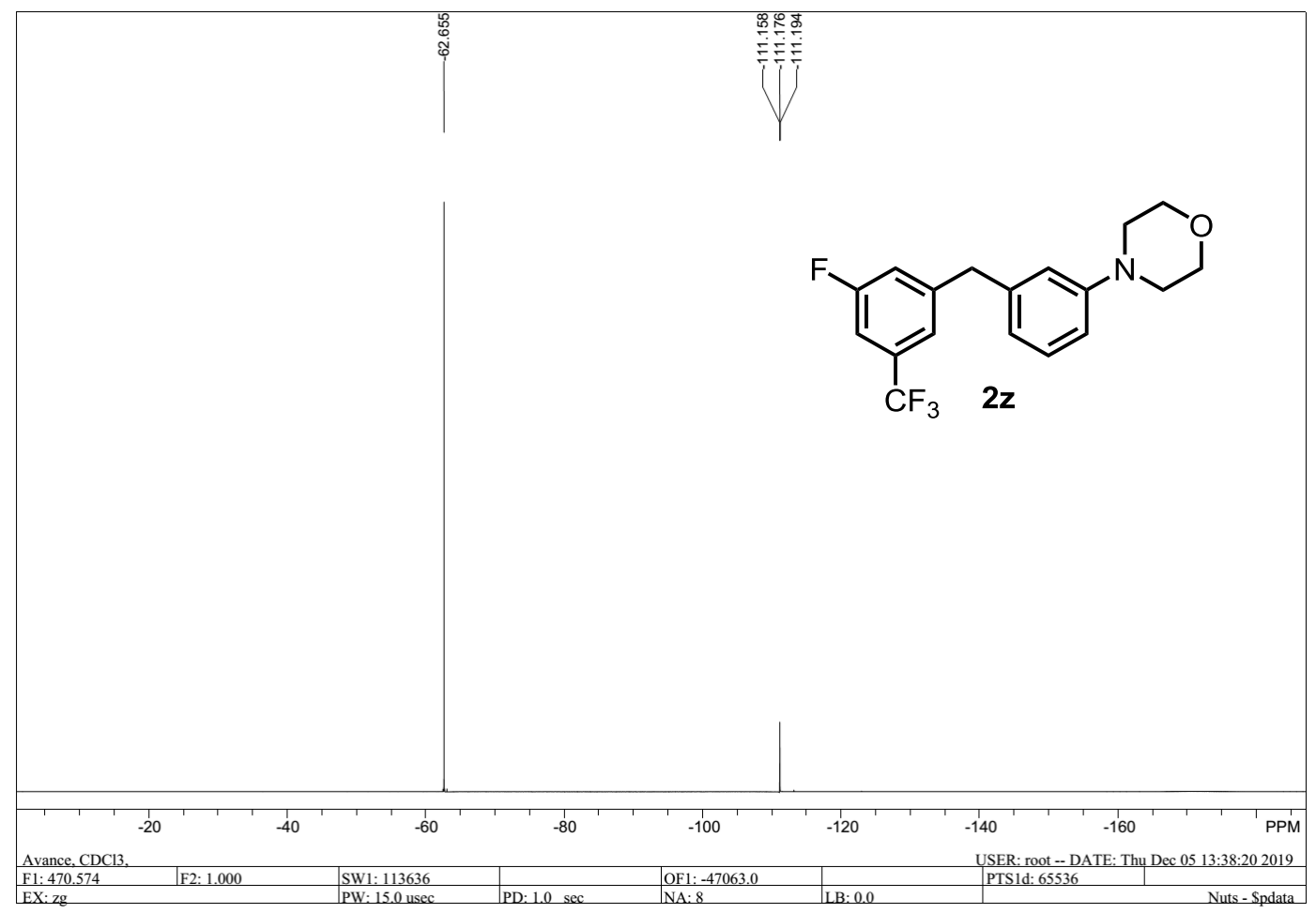


${ }^{1} \mathrm{H}$ NMR (500MHz, $\mathrm{CDCl}_{3}$ ):

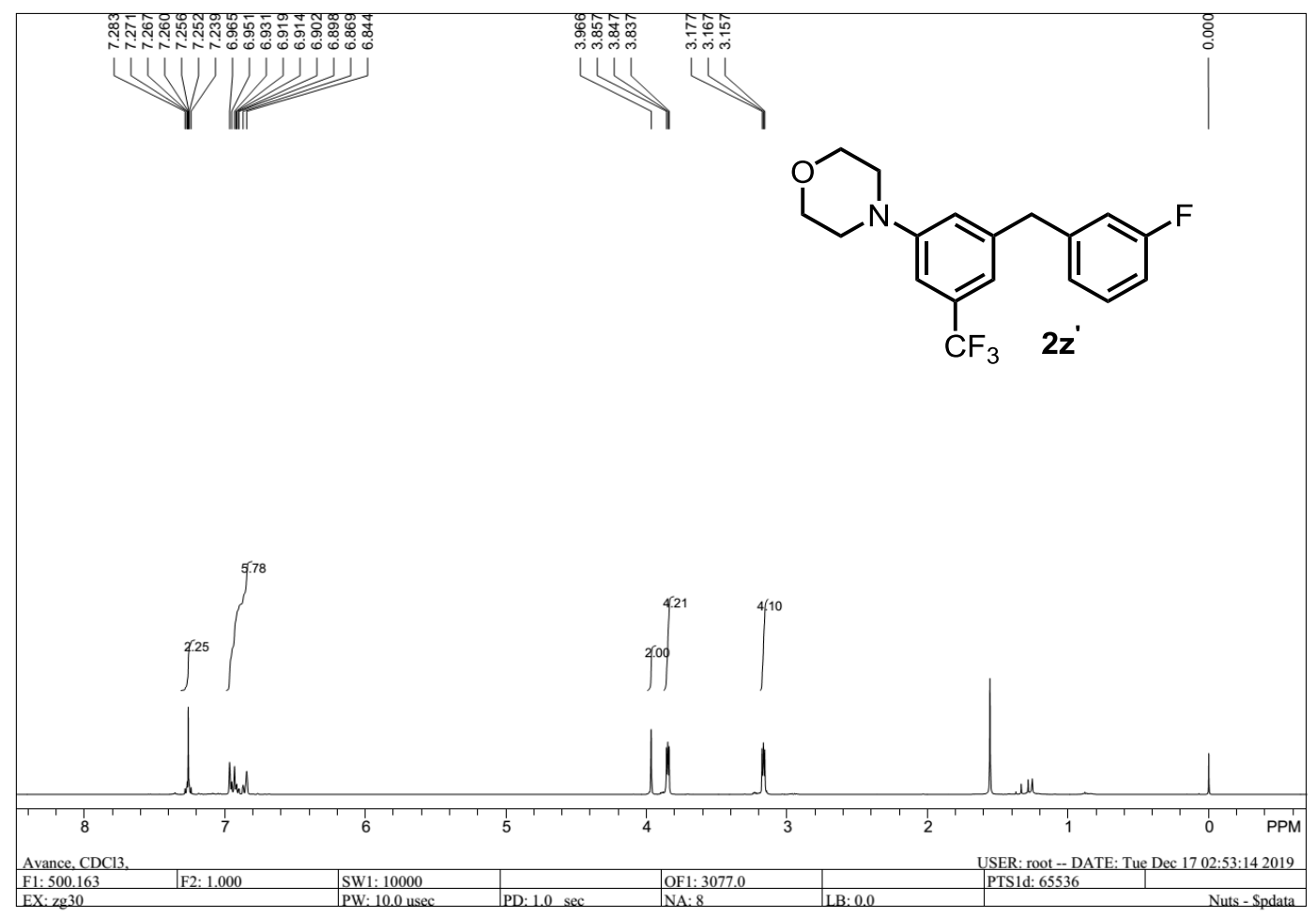

${ }^{13} \mathrm{C}$ NMR $\left(125 \mathrm{MHz}, \mathrm{CDCl}_{3}\right)$ :

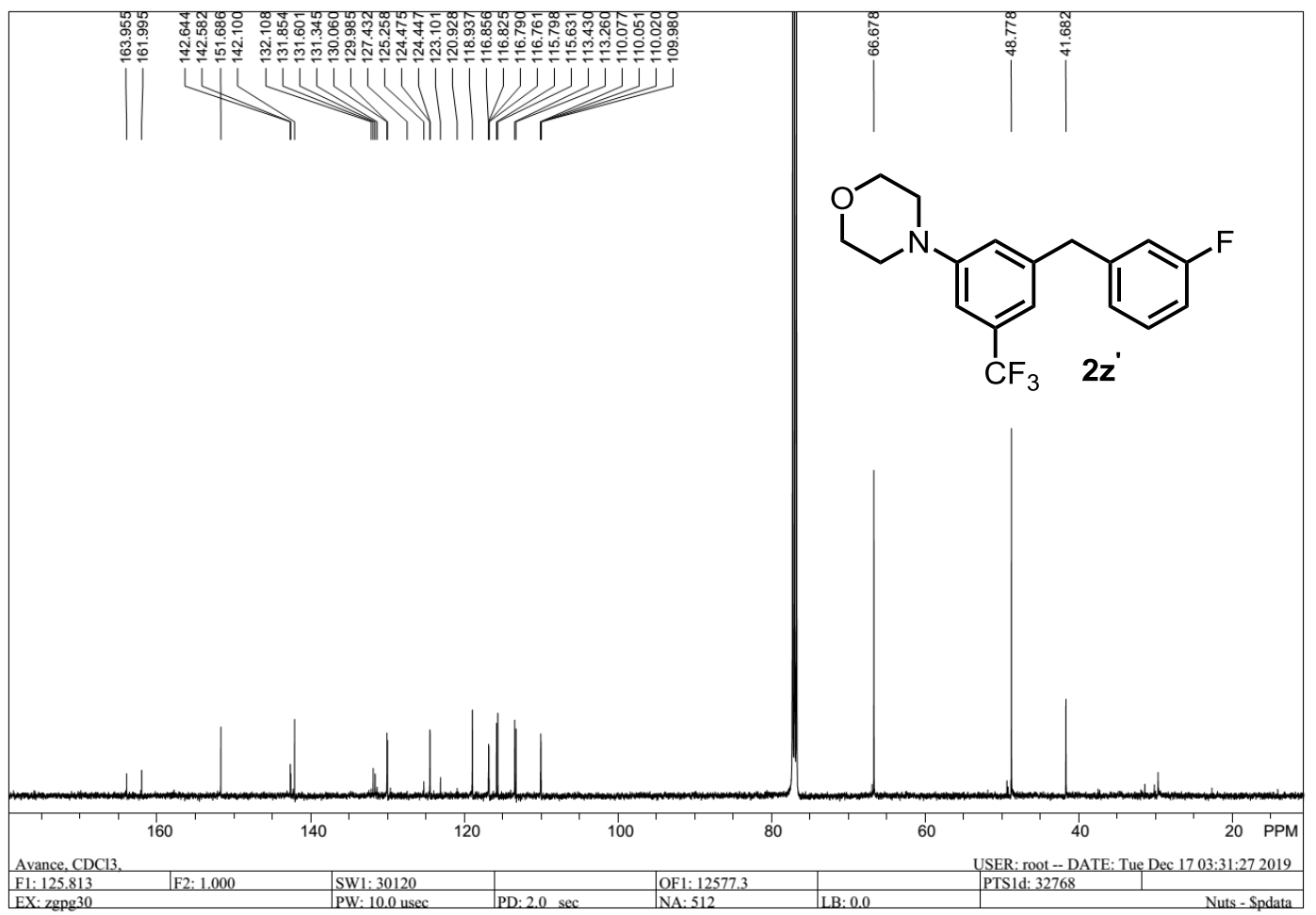


$\left.{ }^{19} \mathrm{~F} \mathrm{NMR} \mathrm{(470MHz,} \mathrm{CDCl}_{3}\right)$ :

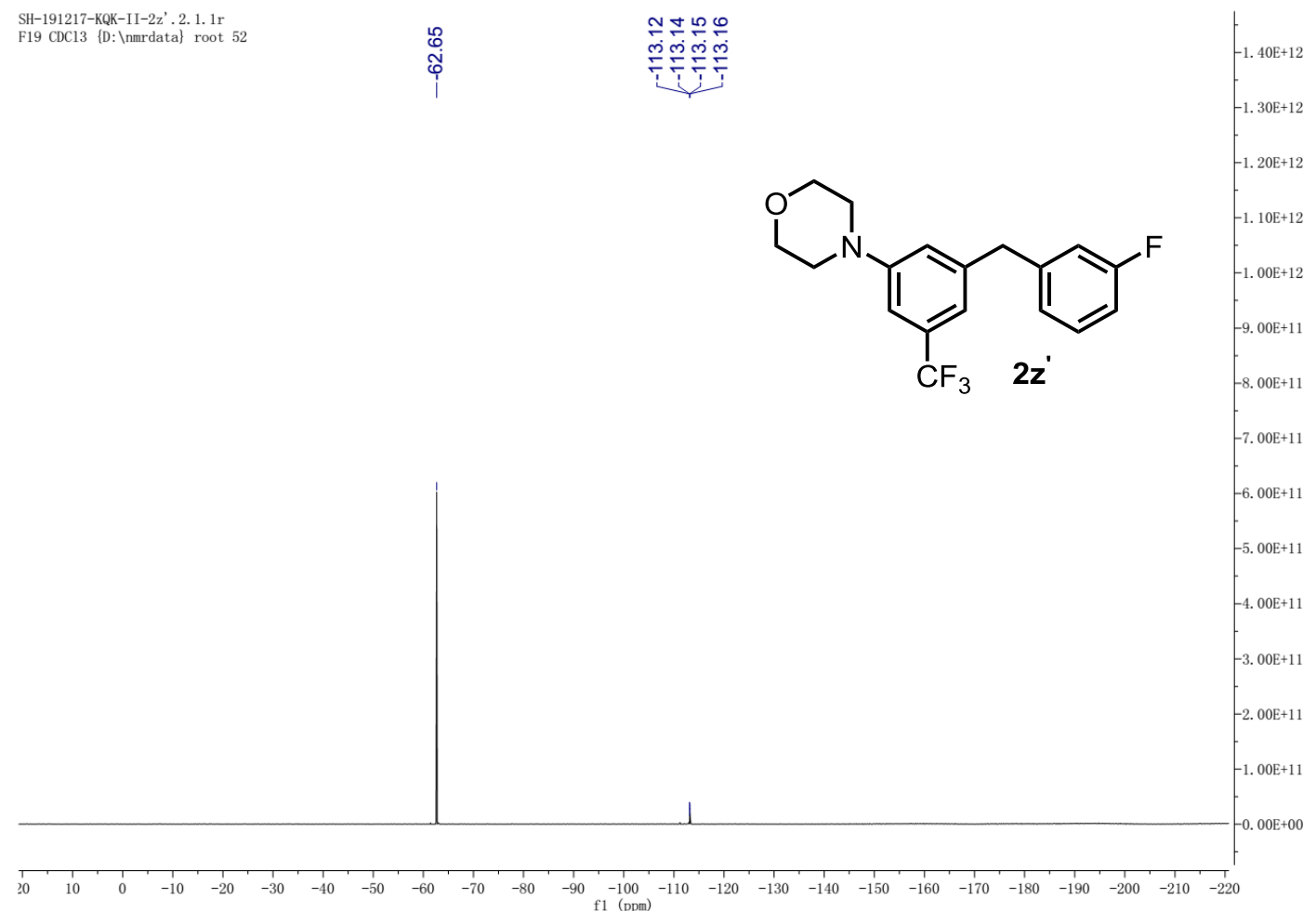


${ }^{1} \mathrm{H} \mathrm{NMR}\left(500 \mathrm{MHz}, \mathrm{CDCl}_{3}\right)$ :

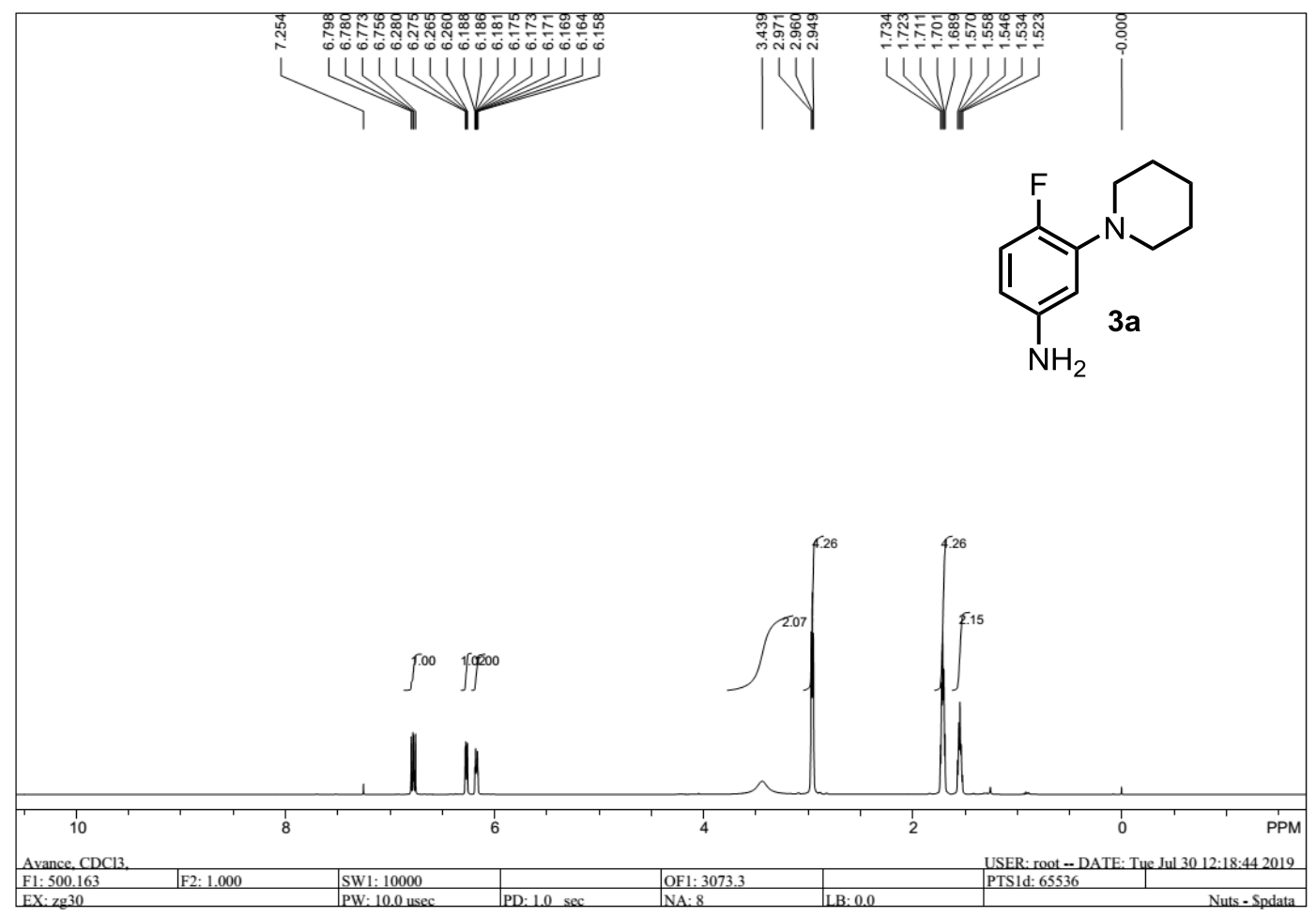

${ }^{13} \mathrm{C}$ NMR $\left(125 \mathrm{MHz}, \mathrm{CDCl}_{3}\right)$ :

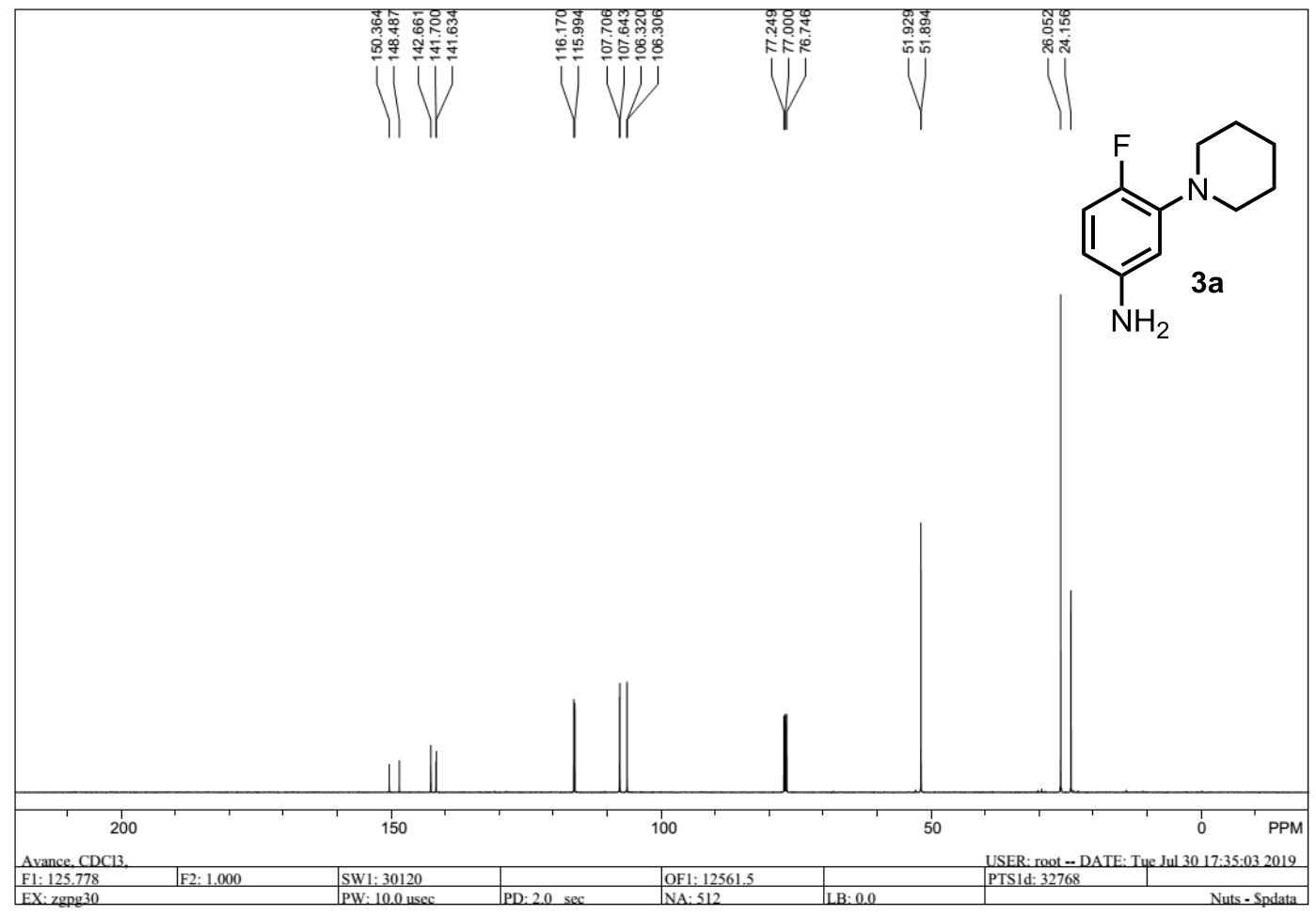


${ }^{19} \mathrm{~F}$ NMR (470 MHz, $\mathrm{CDCl}_{3}$ ):

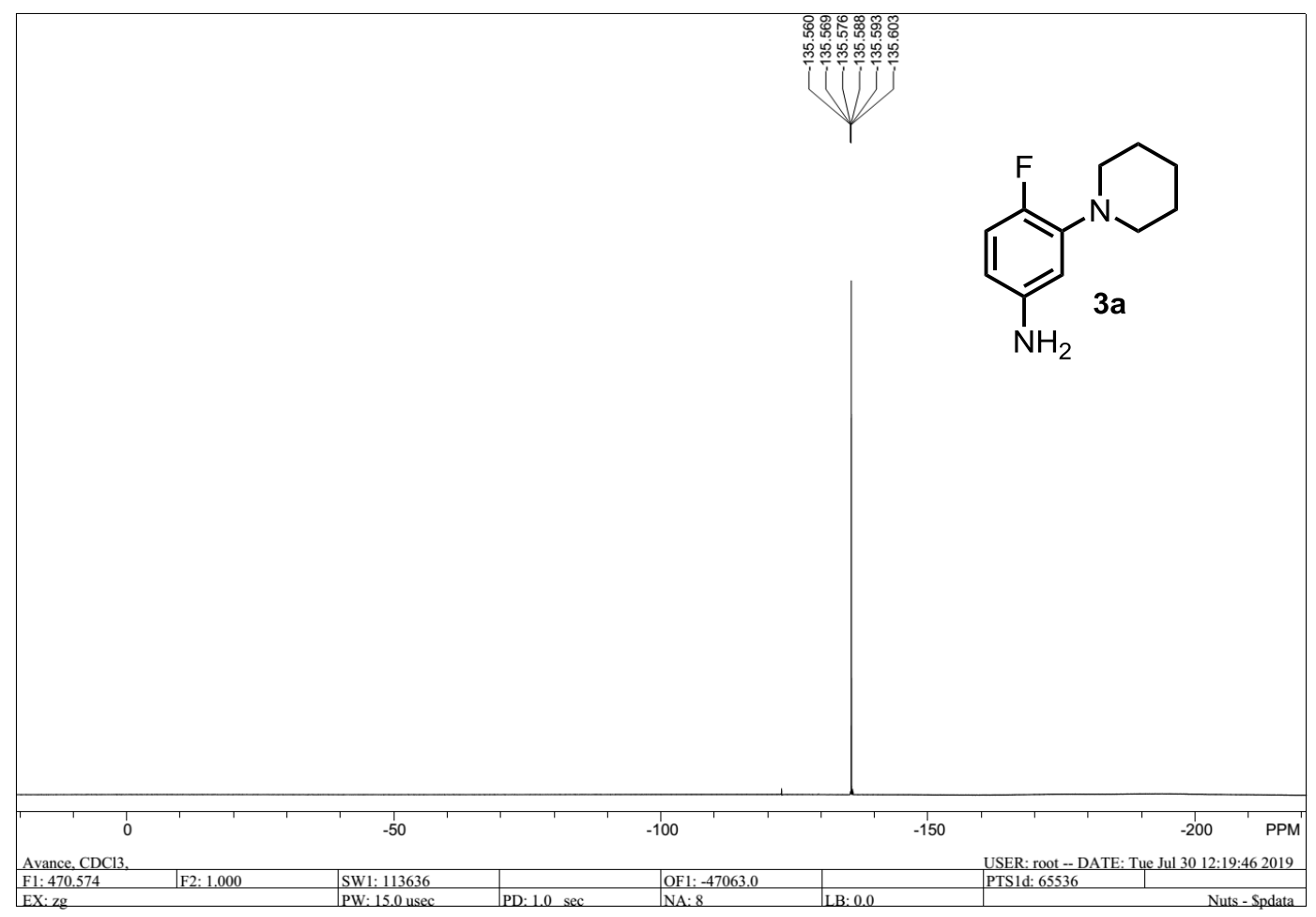


${ }^{1} \mathrm{H}$ NMR (500MHz, $\mathrm{CDCl}_{3}$ ):

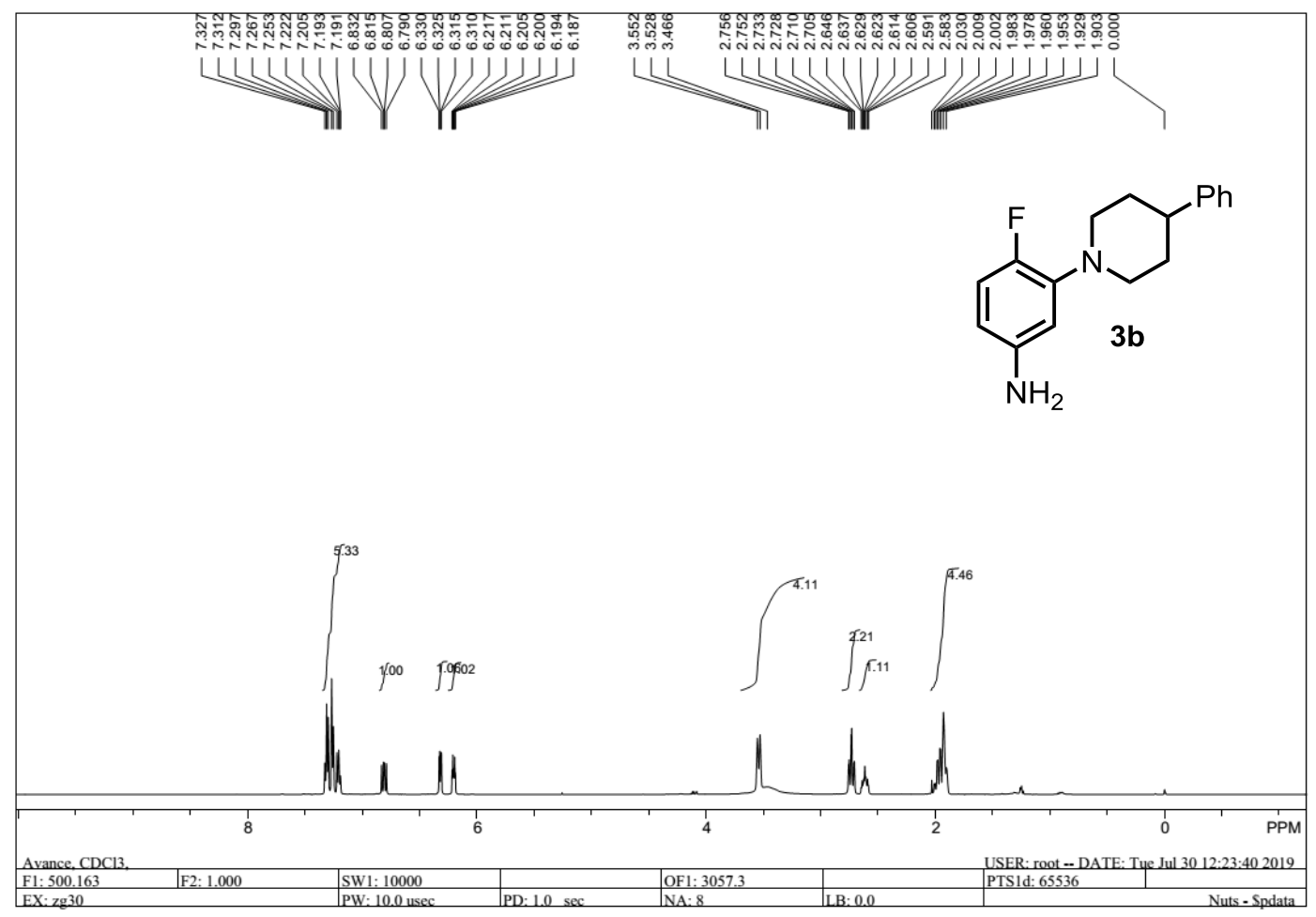

${ }^{13} \mathrm{C}$ NMR $\left(125 \mathrm{MHz}, \mathrm{CDCl}_{3}\right)$ :

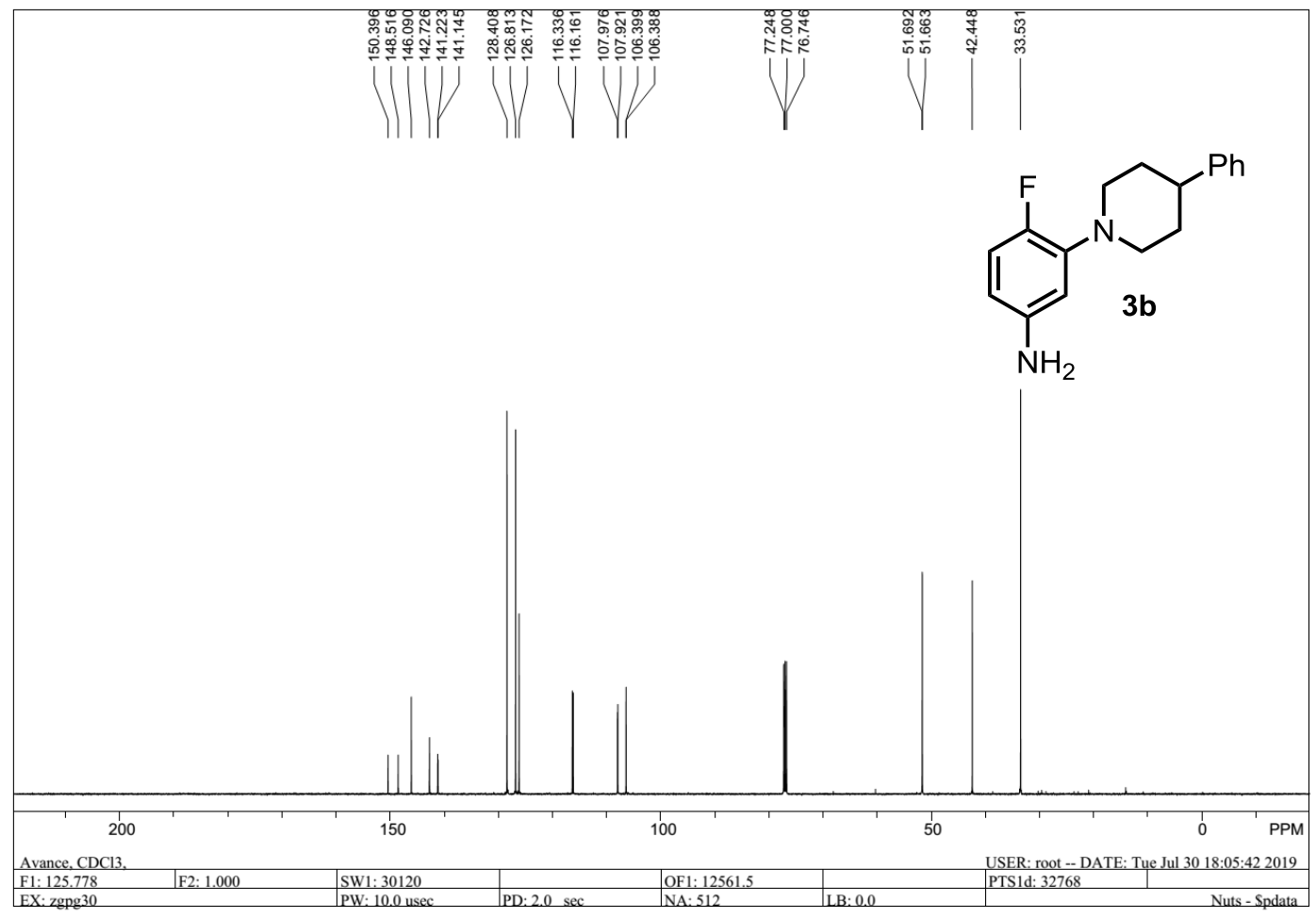


${ }^{19} \mathrm{~F}$ NMR (470 MHz, $\mathrm{CDCl}_{3}$ ):

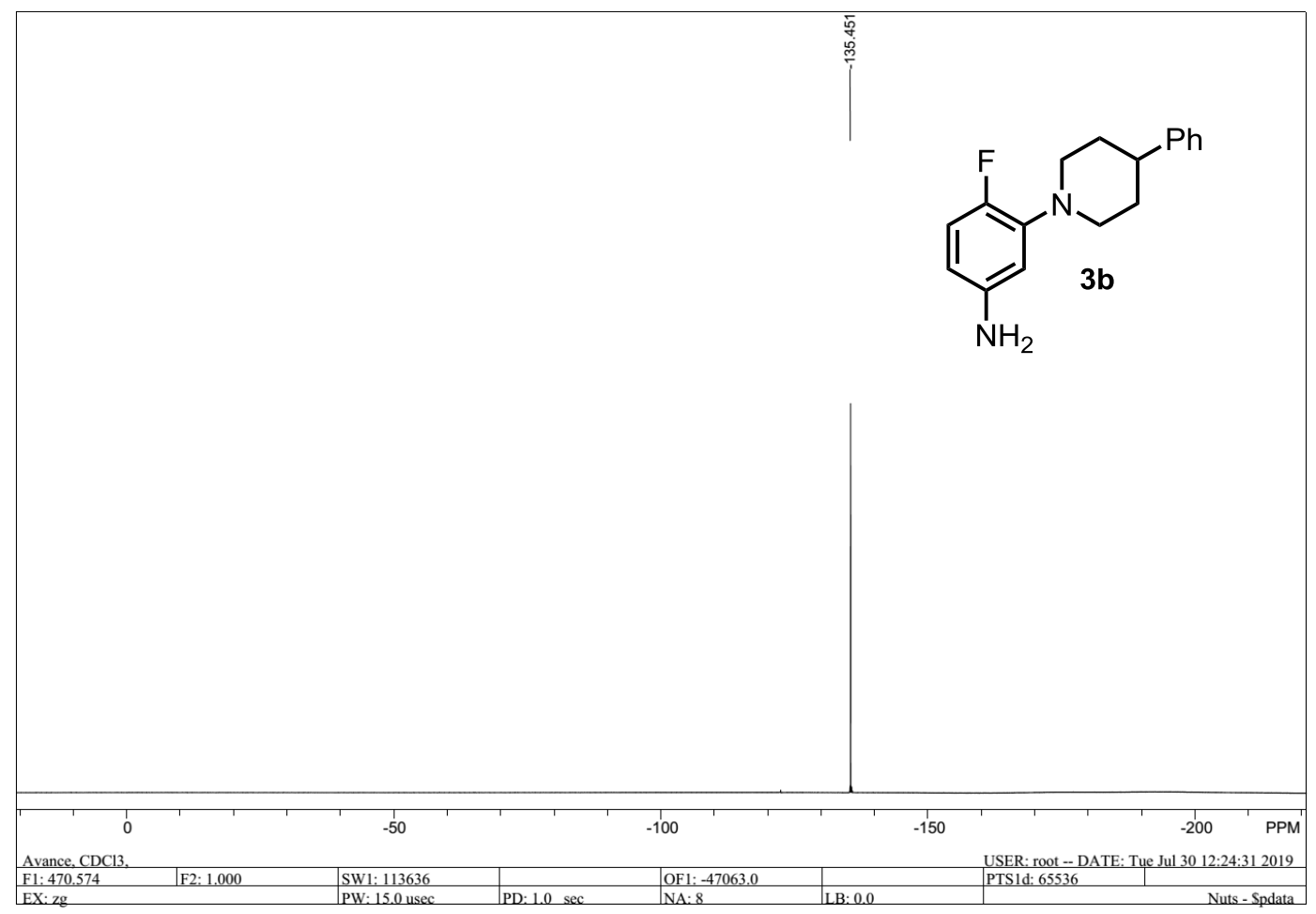


${ }^{1} \mathrm{H}$ NMR $\left(500 \mathrm{MHz}, \mathrm{CDCl}_{3}\right)$ :

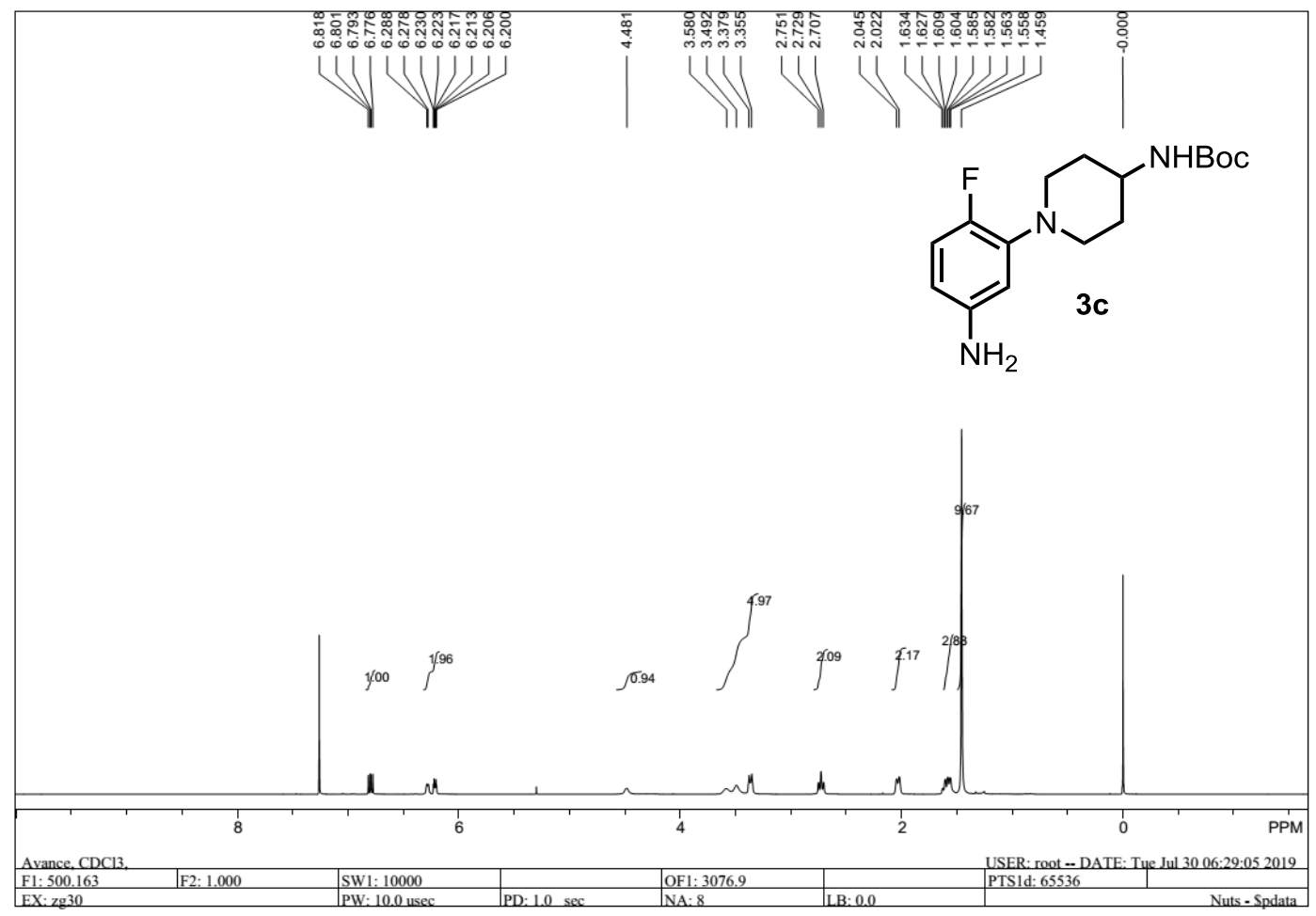

${ }^{13} \mathrm{C}$ NMR $\left(125 \mathrm{MHz}, \mathrm{CDCl}_{3}\right)$ :

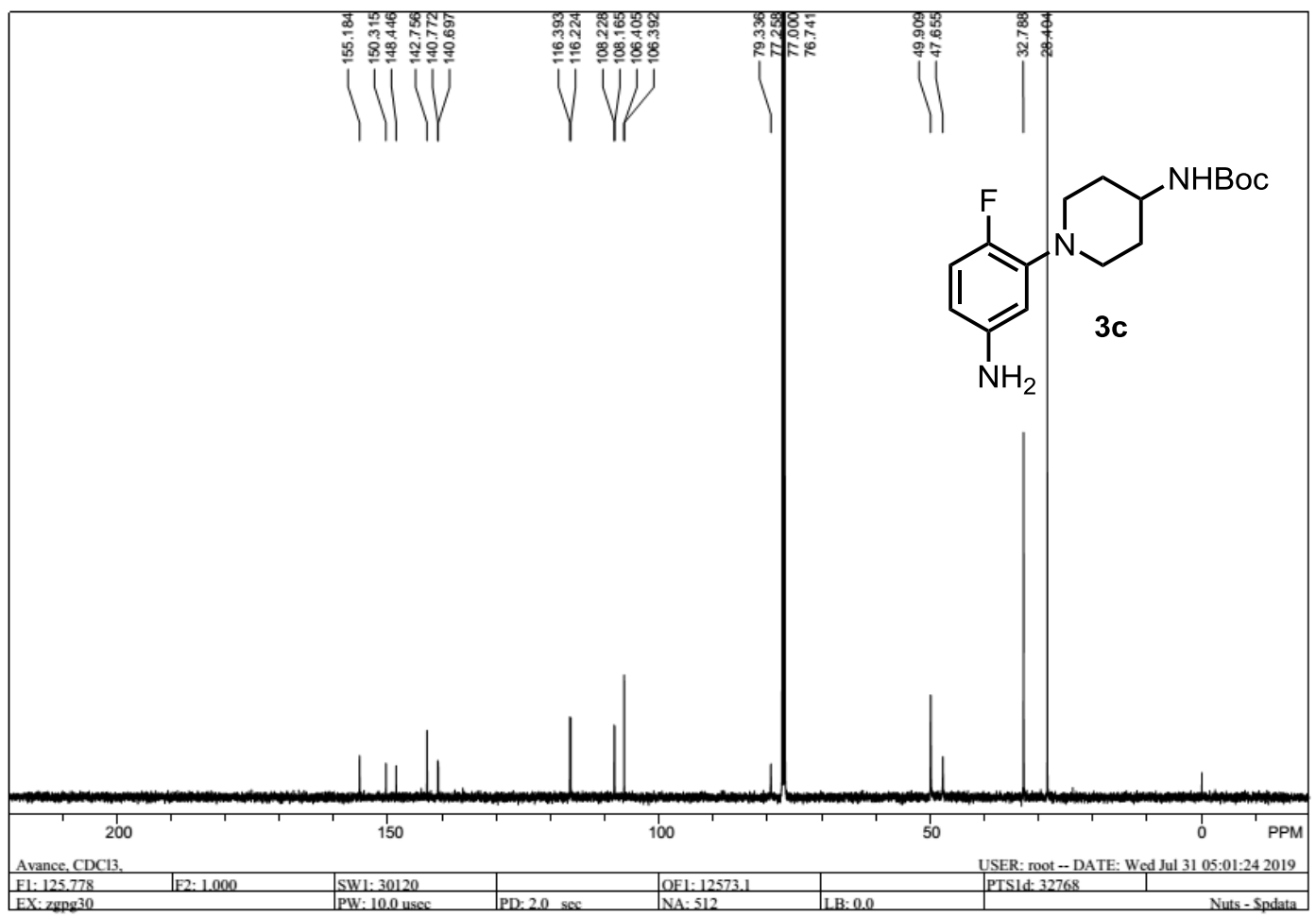


${ }^{19} \mathrm{~F}$ NMR (470 MHz, $\mathrm{CDCl}_{3}$ ):

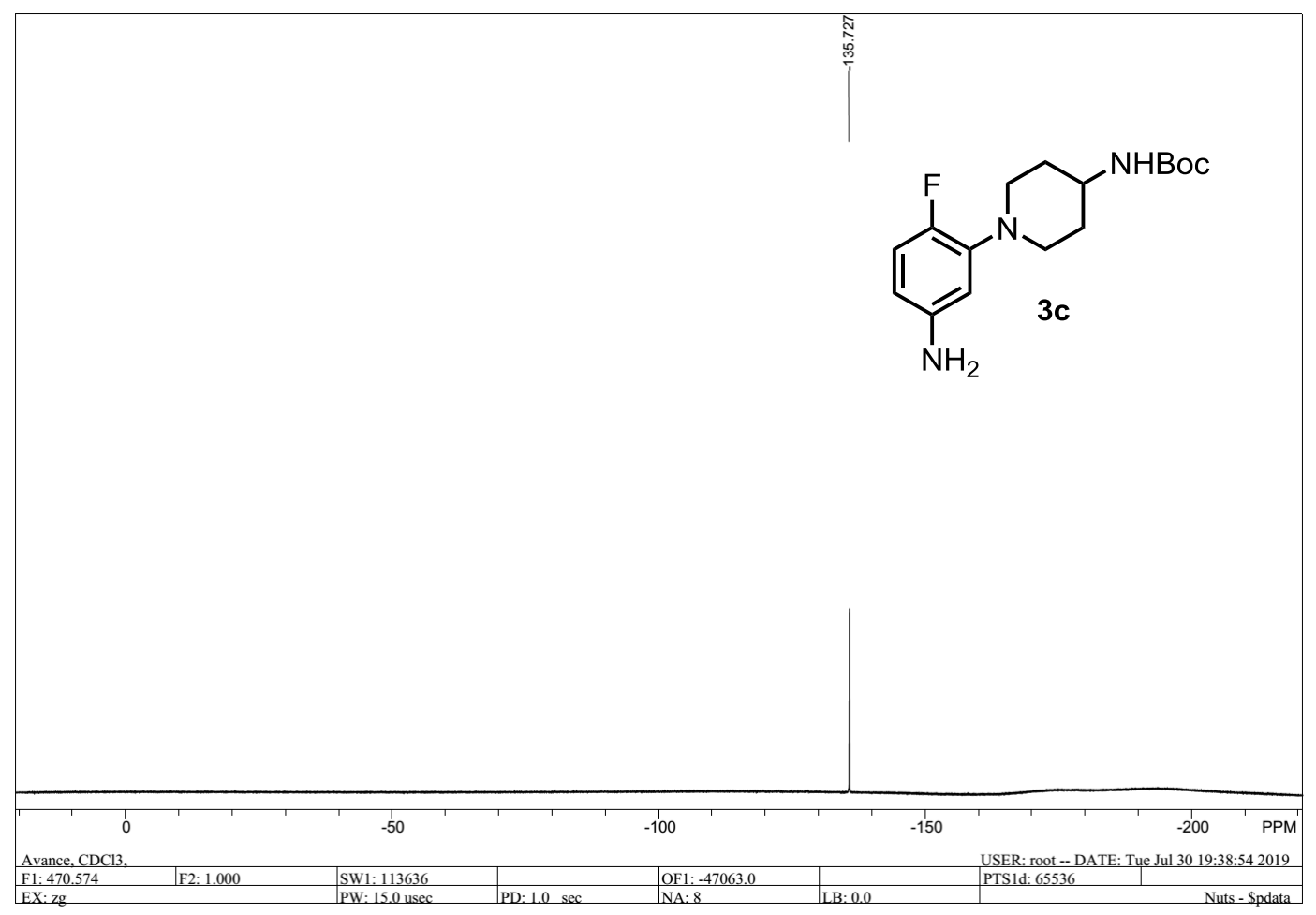


${ }^{1} \mathrm{H}$ NMR (500MHz, $\mathrm{CDCl}_{3}$ ):

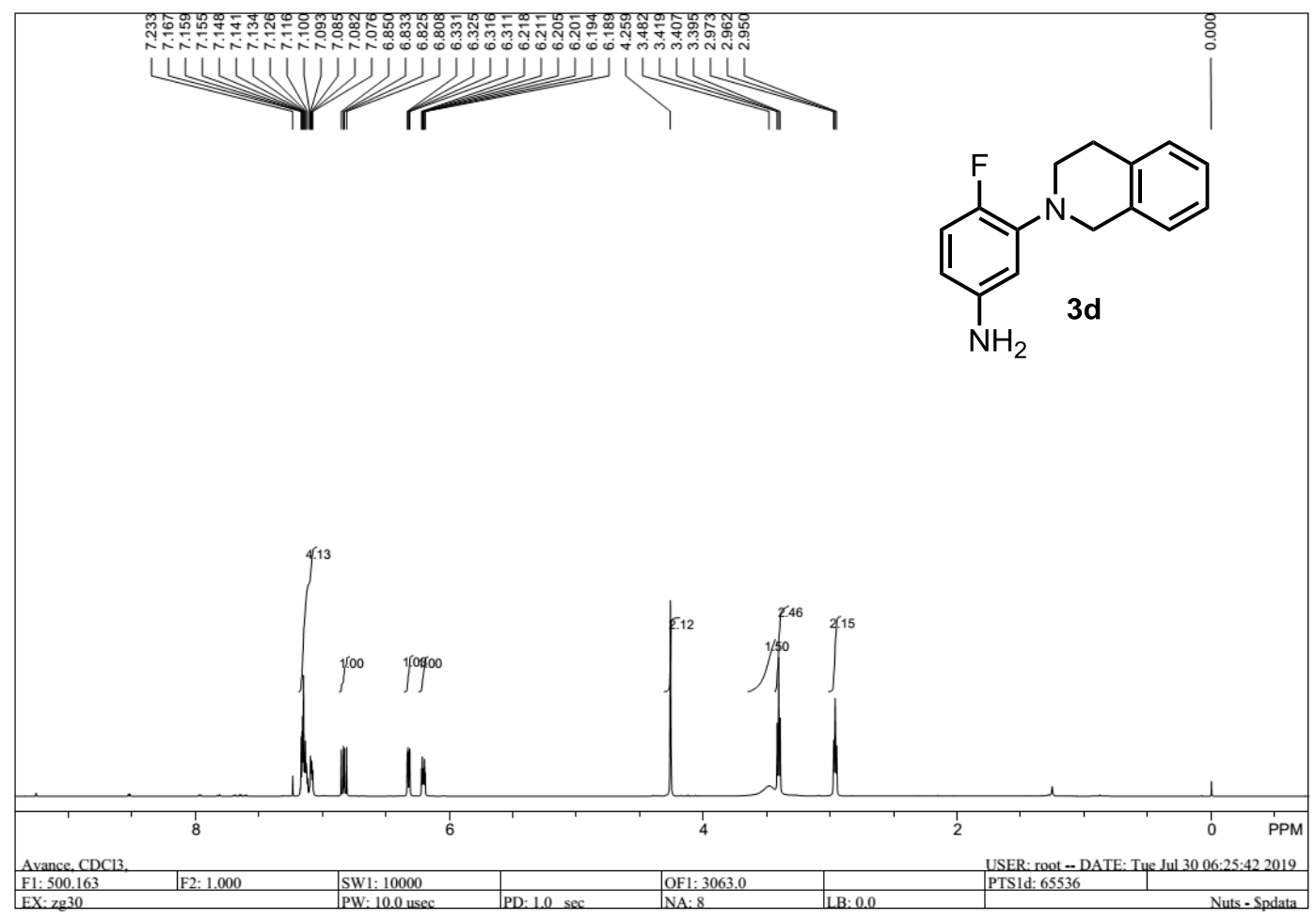

${ }^{13} \mathrm{C}$ NMR $\left(125 \mathrm{MHz}, \mathrm{CDCl}_{3}\right)$ :

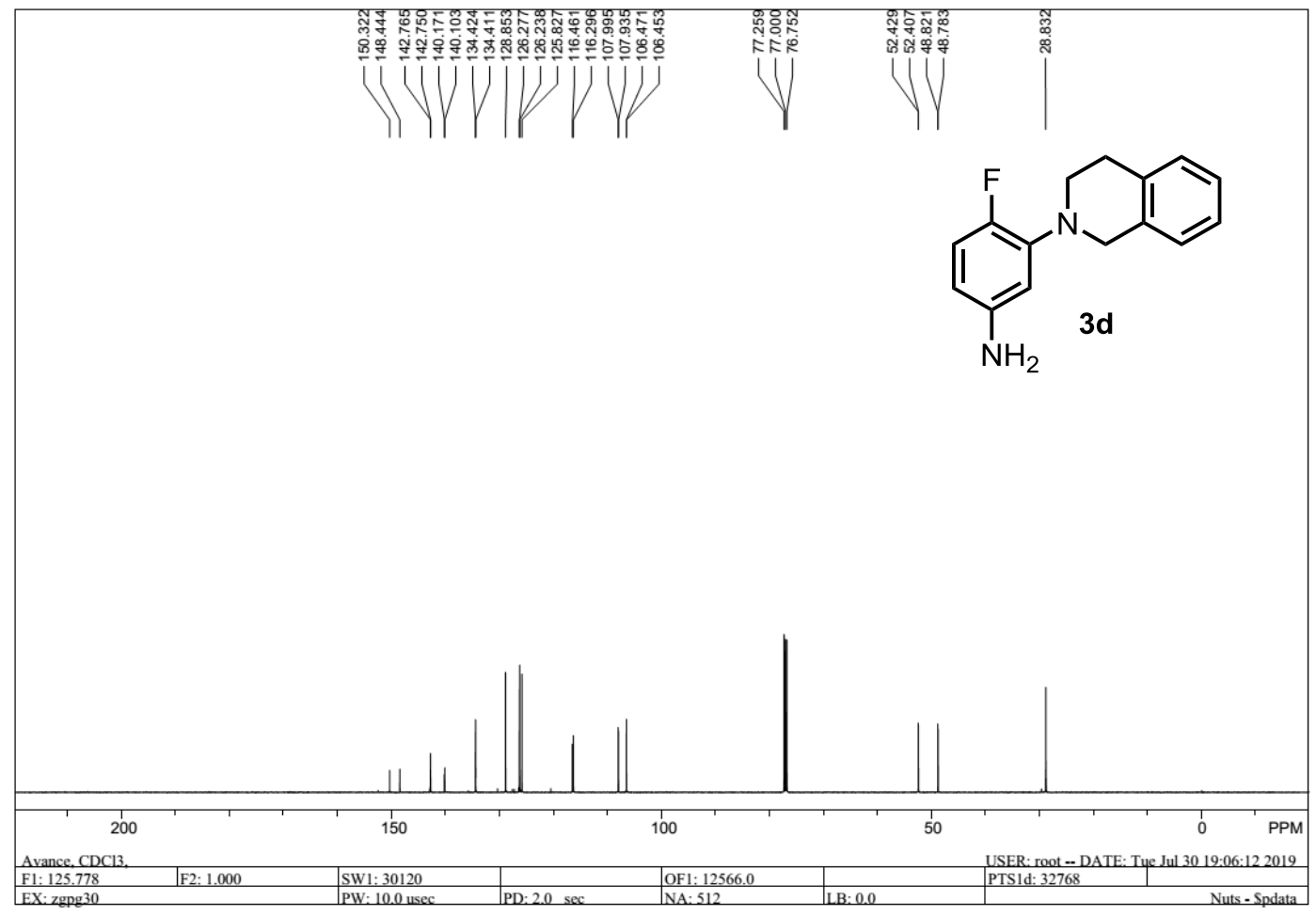


${ }^{19} \mathrm{~F}$ NMR (470 MHz, $\mathrm{CDCl}_{3}$ ):

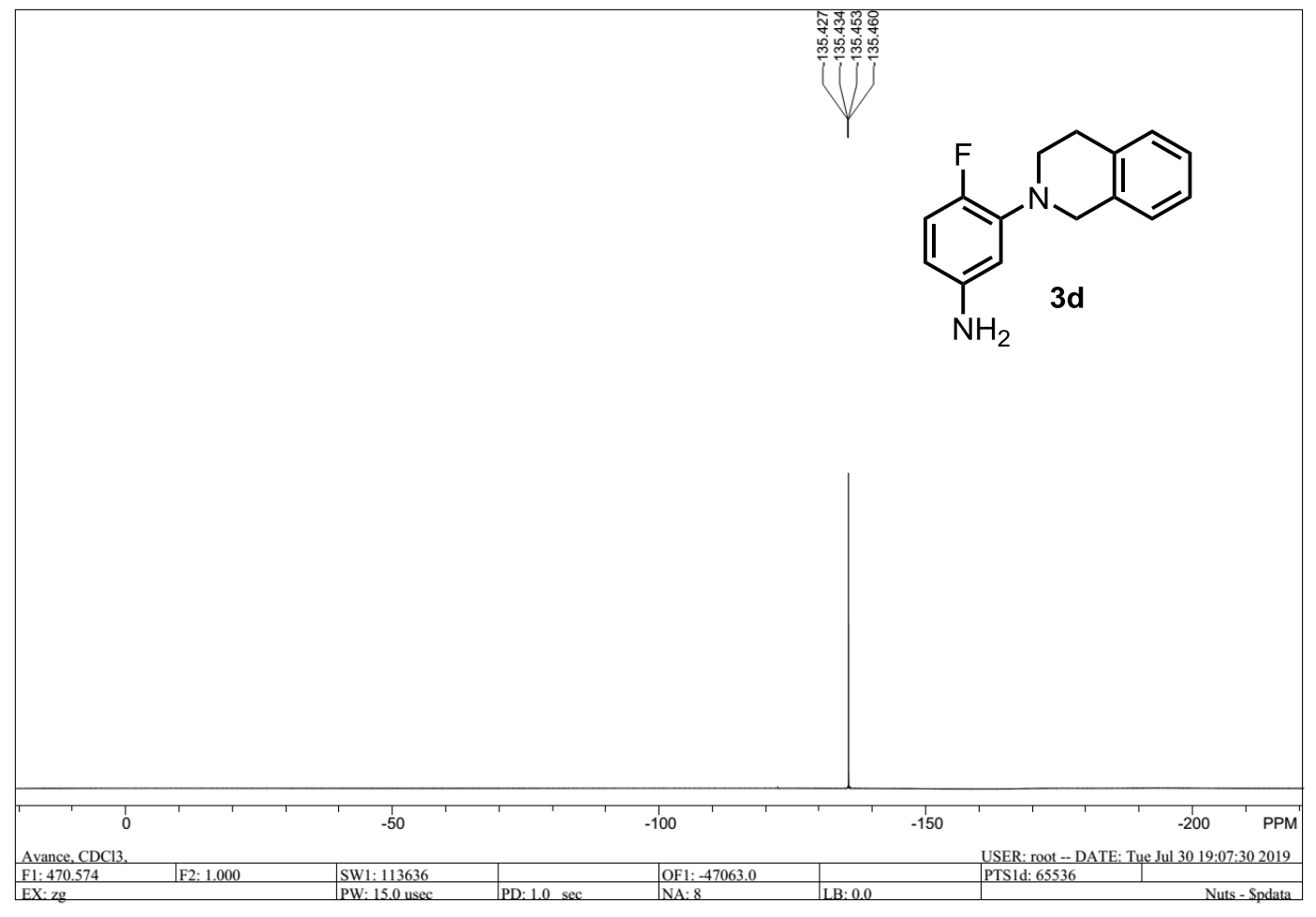


${ }^{1} \mathrm{H}$ NMR (500MHz, $\mathrm{CDCl}_{3}$ ):

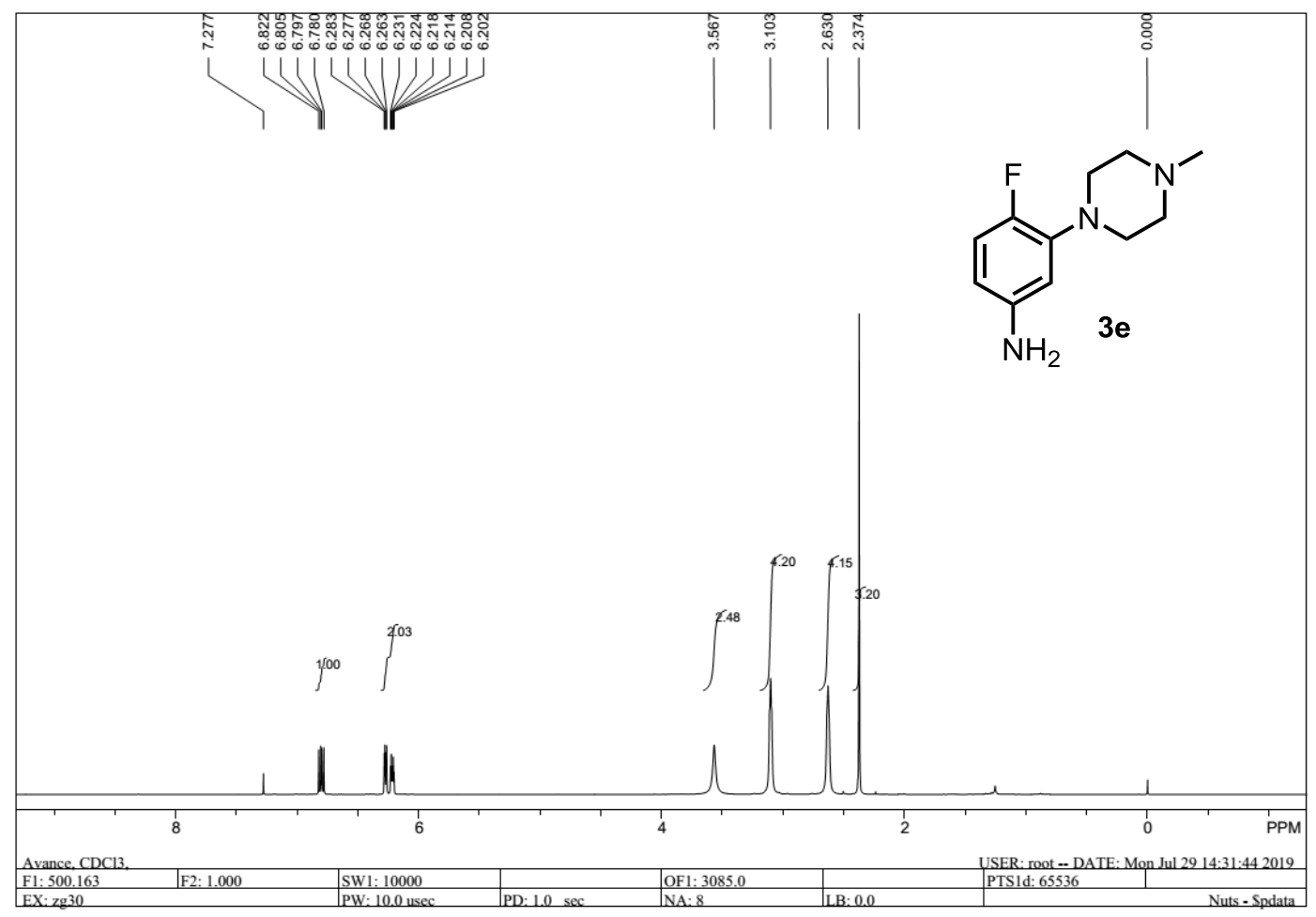

${ }^{13} \mathrm{C}$ NMR $\left(125 \mathrm{MHz}, \mathrm{CDCl}_{3}\right)$ :

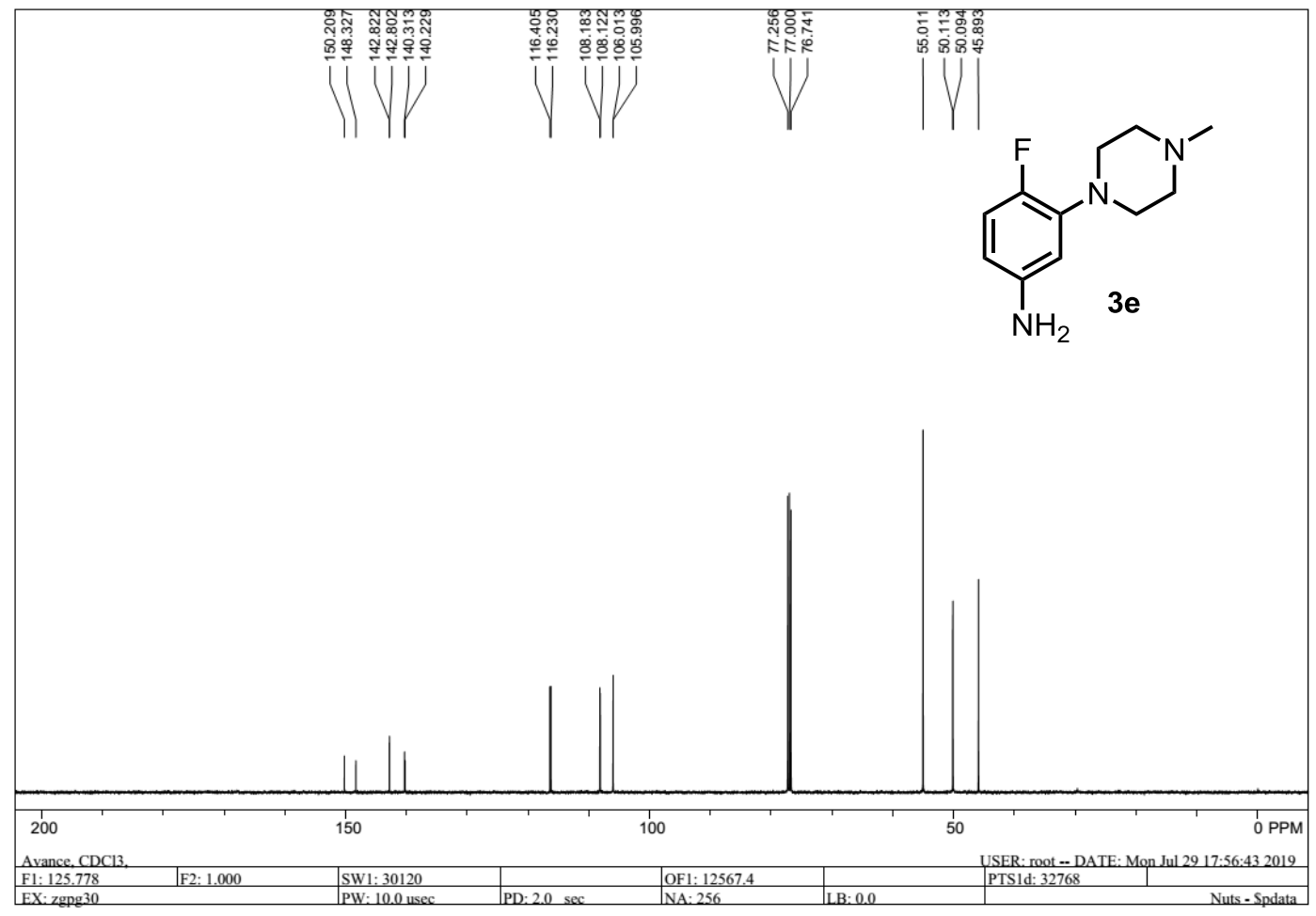


${ }^{19} \mathrm{~F}$ NMR (470 MHz, $\mathrm{CDCl}_{3}$ ):

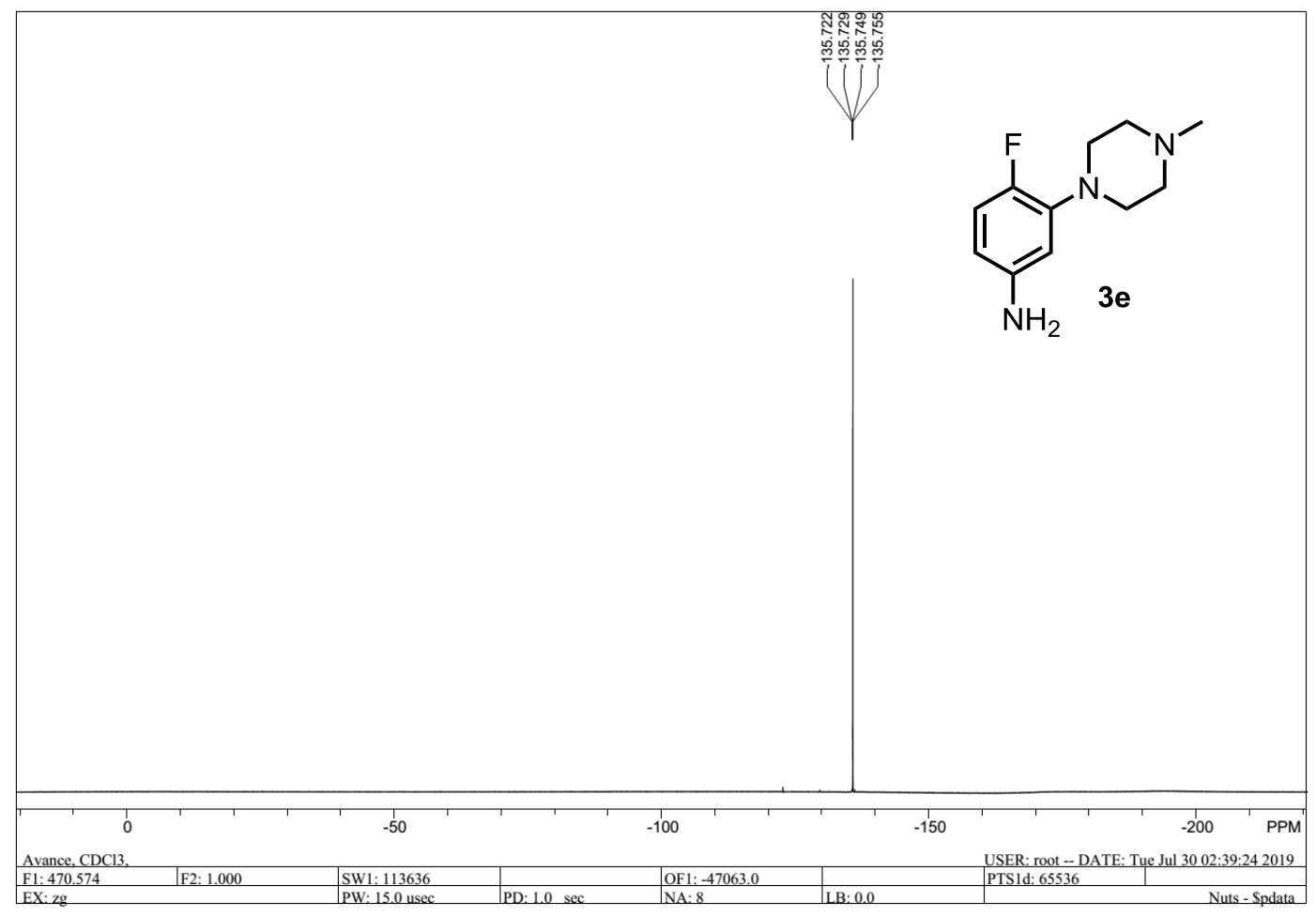


${ }^{1} \mathrm{H}$ NMR (500MHz, $\mathrm{CDCl}_{3}$ ):

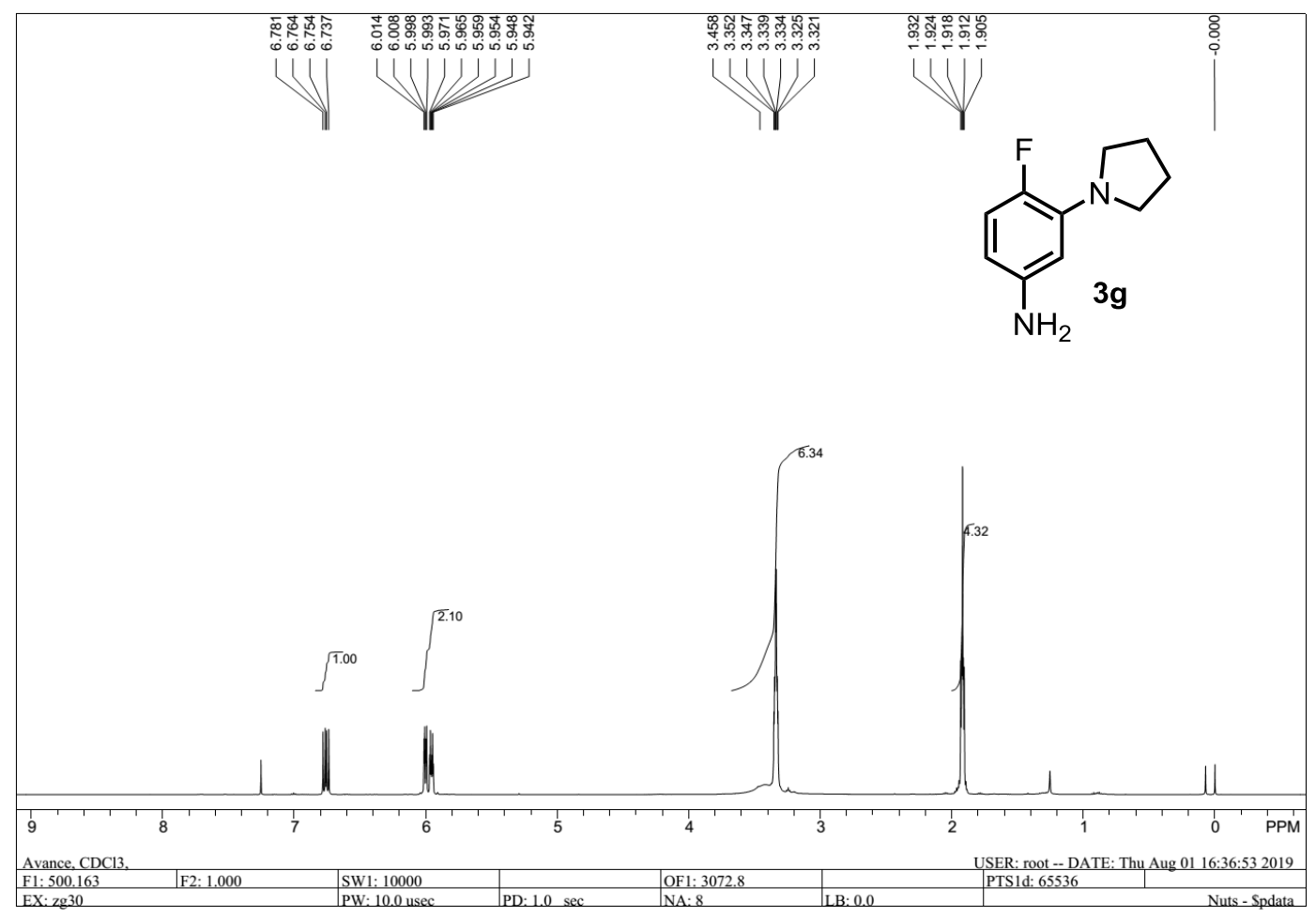

${ }^{13} \mathrm{C}$ NMR $\left(125 \mathrm{MHz}, \mathrm{CDCl}_{3}\right)$ :

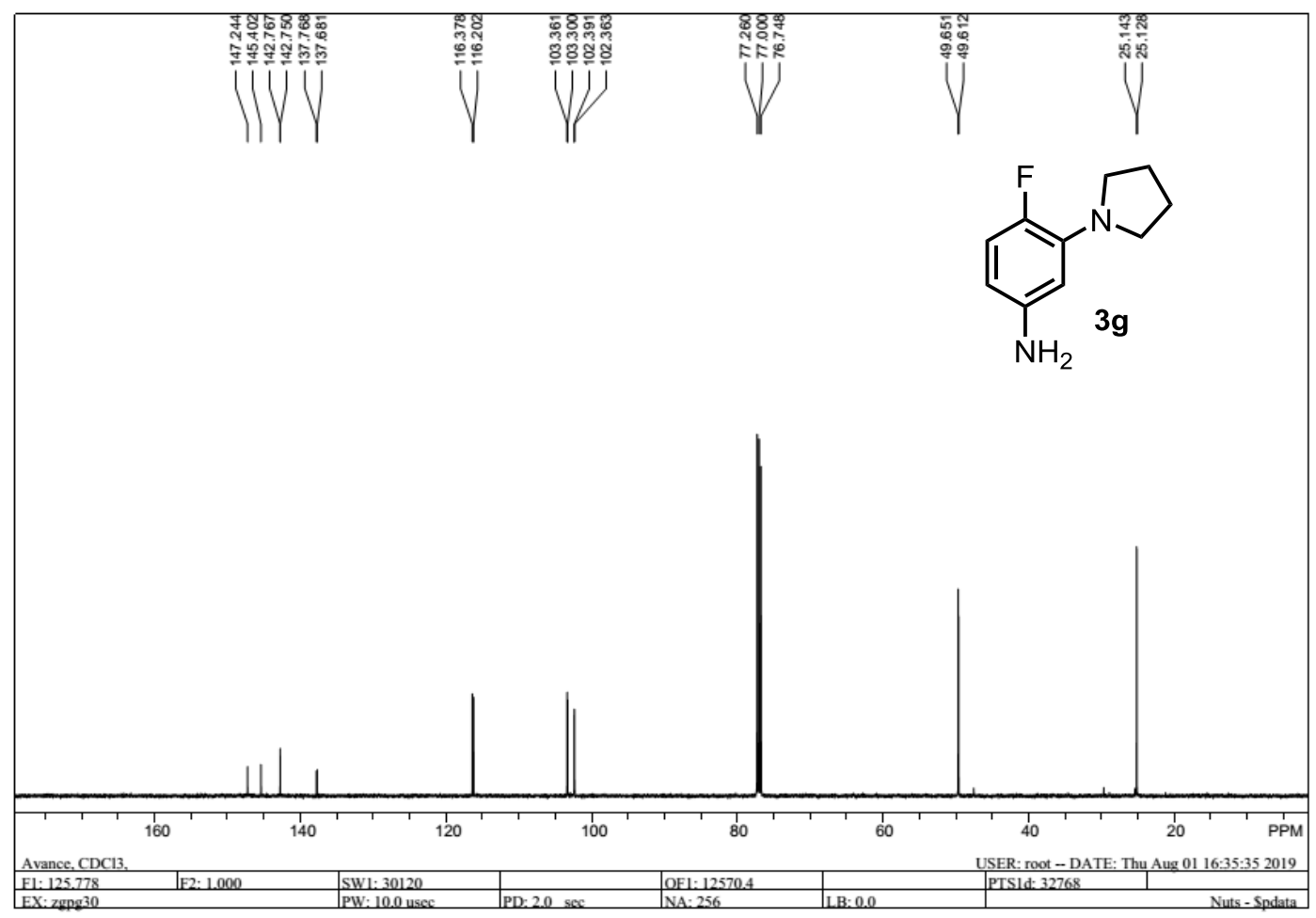


${ }^{19} \mathrm{~F}$ NMR (470 MHz, $\mathrm{CDCl}_{3}$ ):

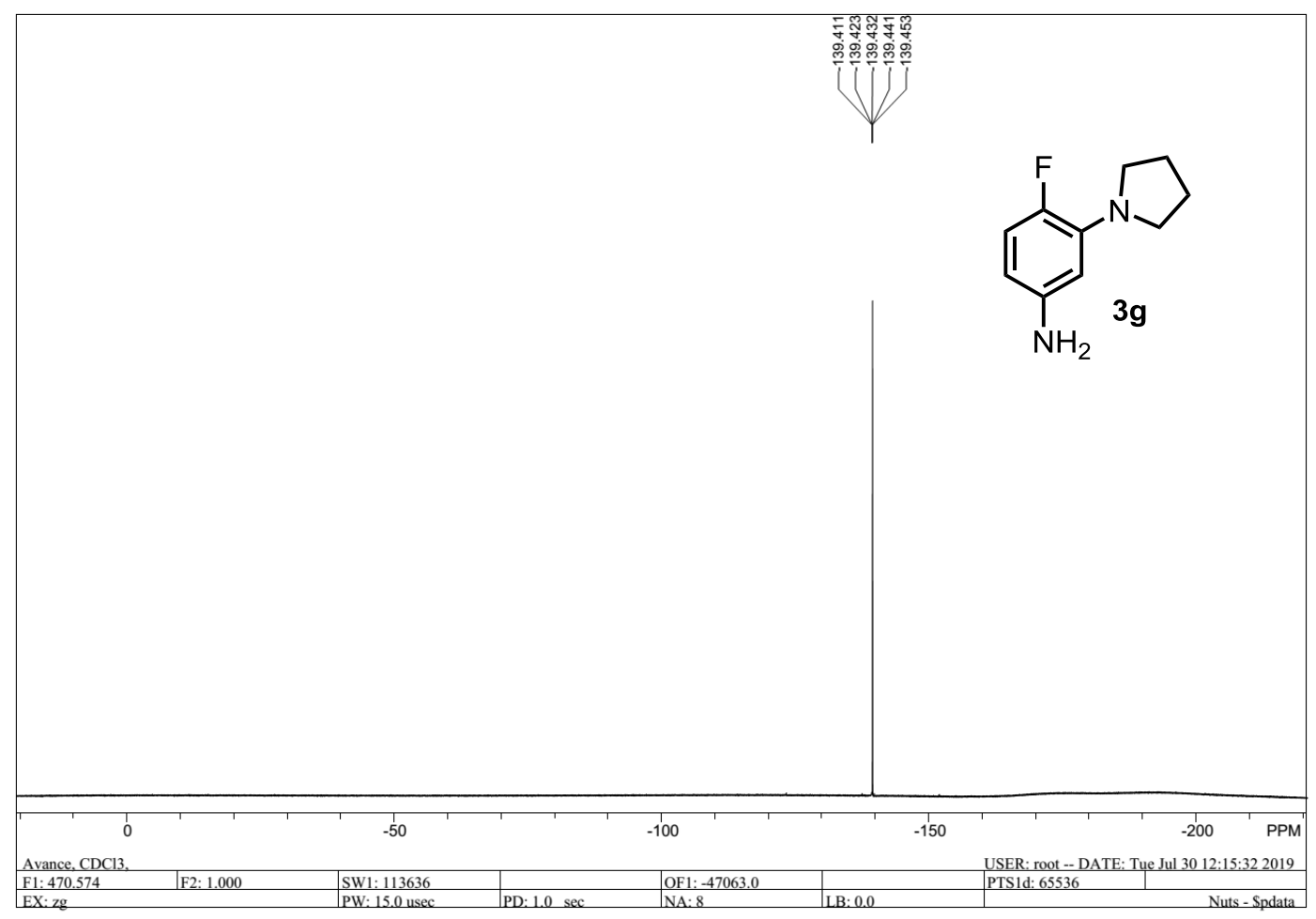


${ }^{1} \mathrm{H}$ NMR $\left(500 \mathrm{MHz}, \mathrm{CDCl}_{3}\right)$ :

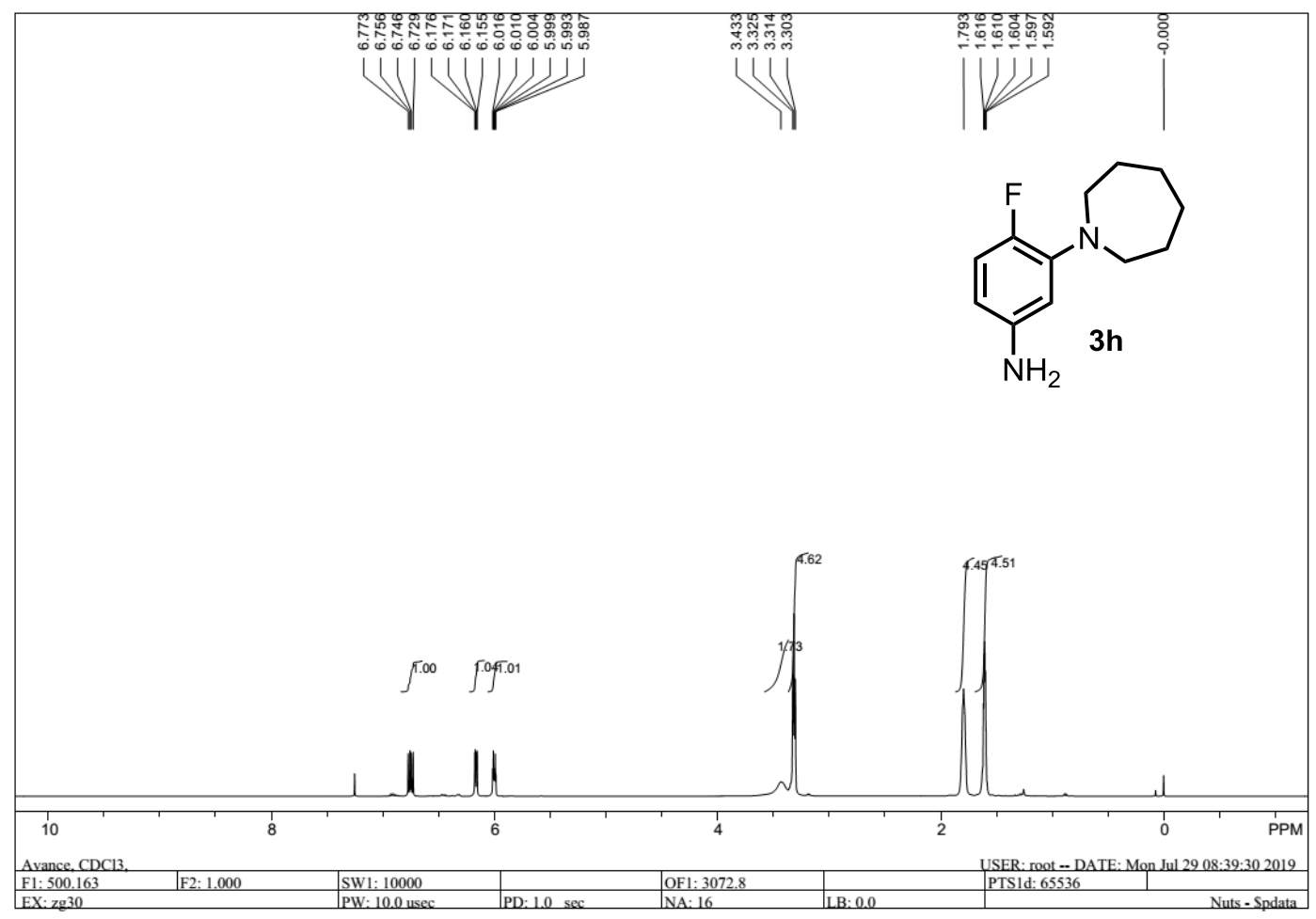

${ }^{13} \mathrm{C}$ NMR $\left(125 \mathrm{MHz}, \mathrm{CDCl}_{3}\right)$ :

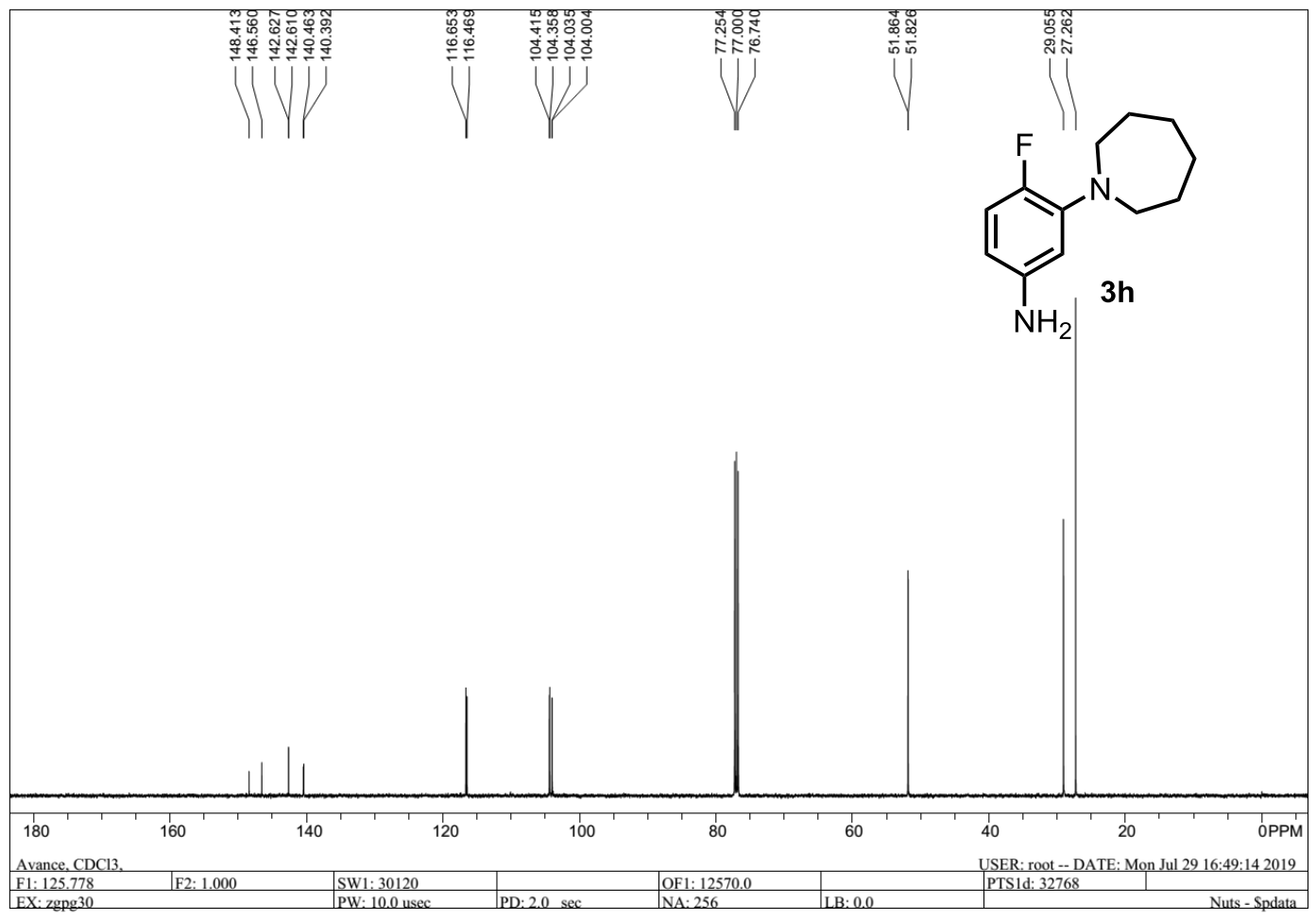


${ }^{19} \mathrm{~F}$ NMR (470 MHz, $\mathrm{CDCl}_{3}$ ):

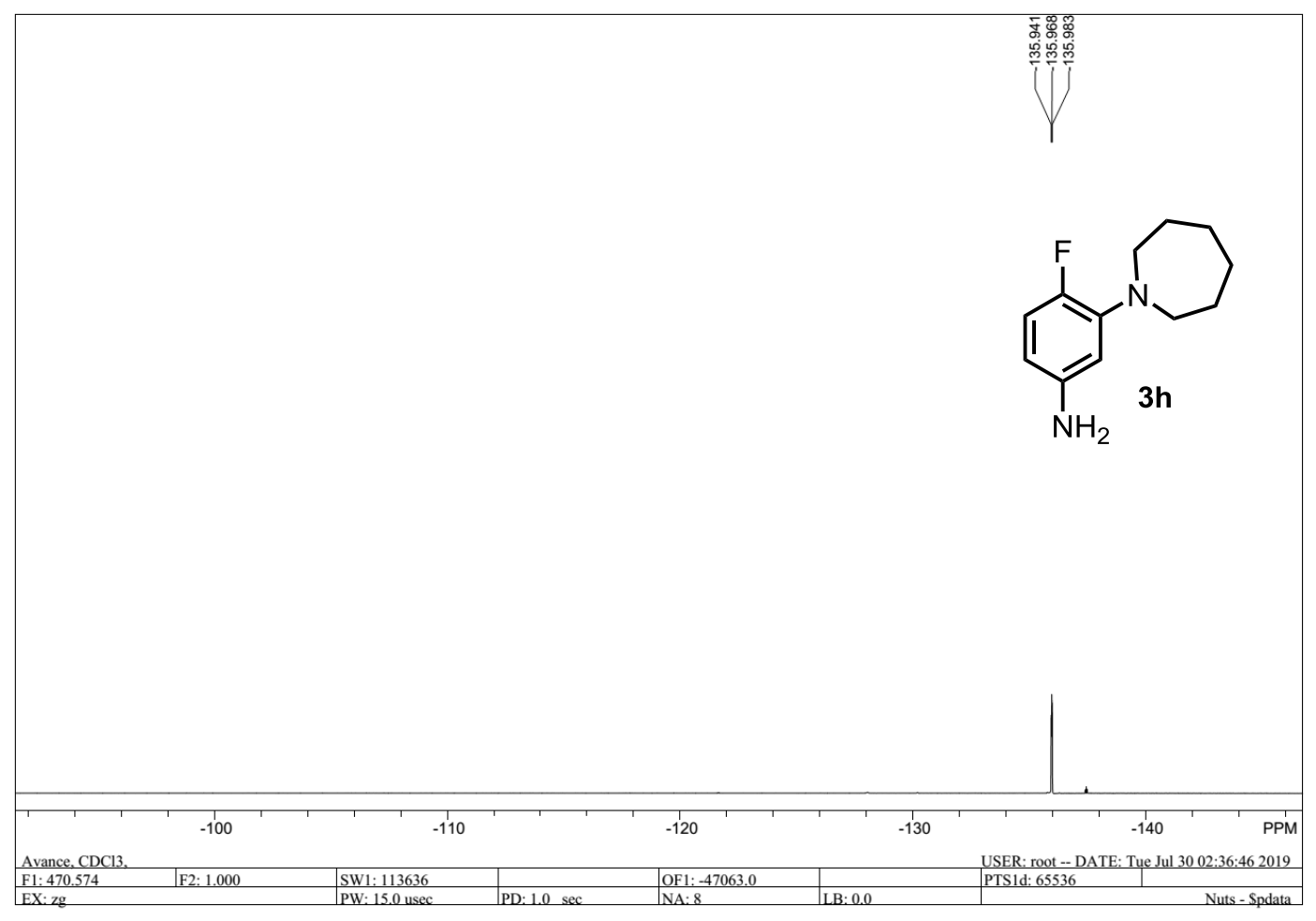


${ }^{1} \mathrm{H}$ NMR (500MHz, $\mathrm{CDCl}_{3}$ ):

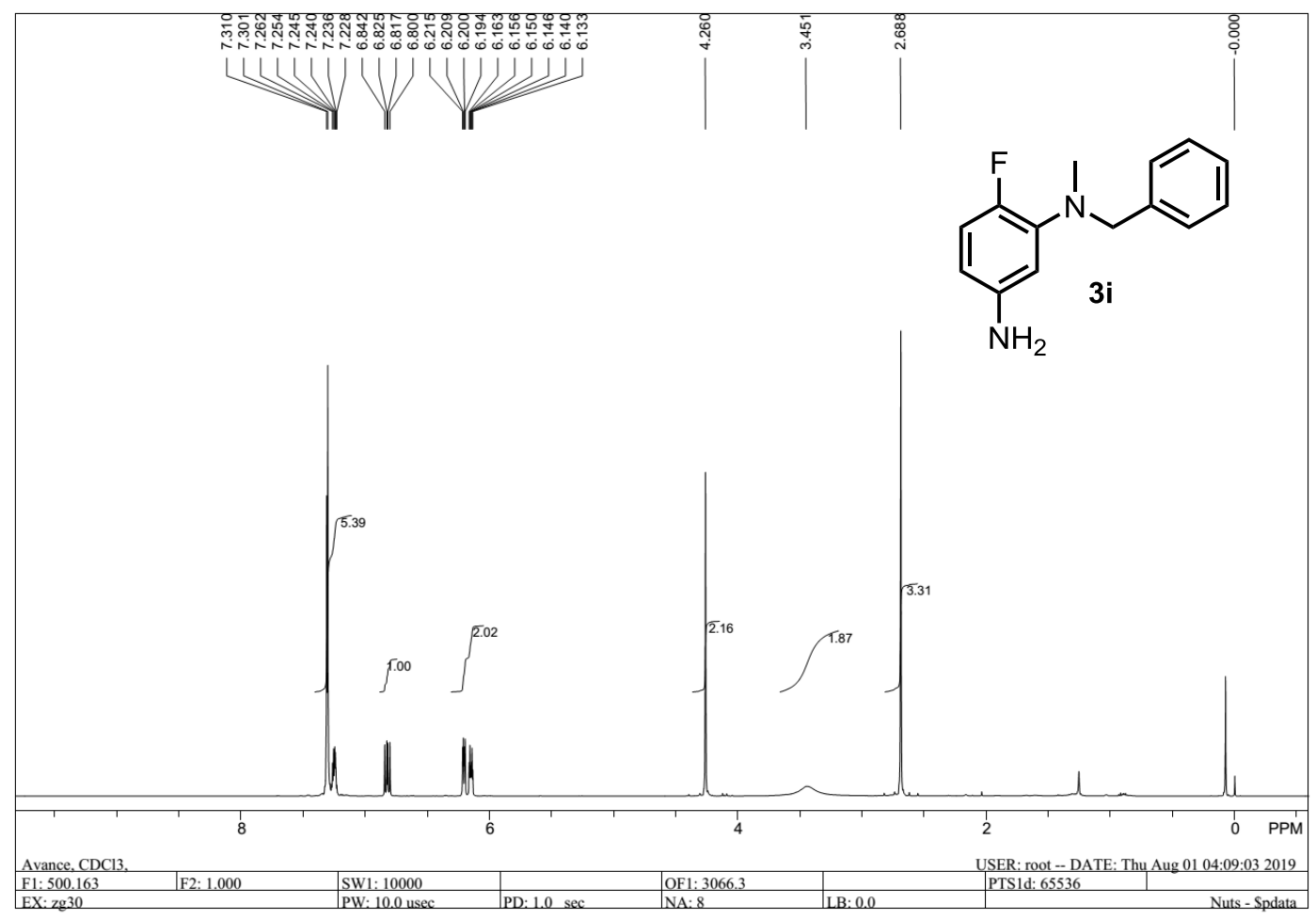

${ }^{13} \mathrm{C}$ NMR $\left(125 \mathrm{MHz}, \mathrm{CDCl}_{3}\right)$ :

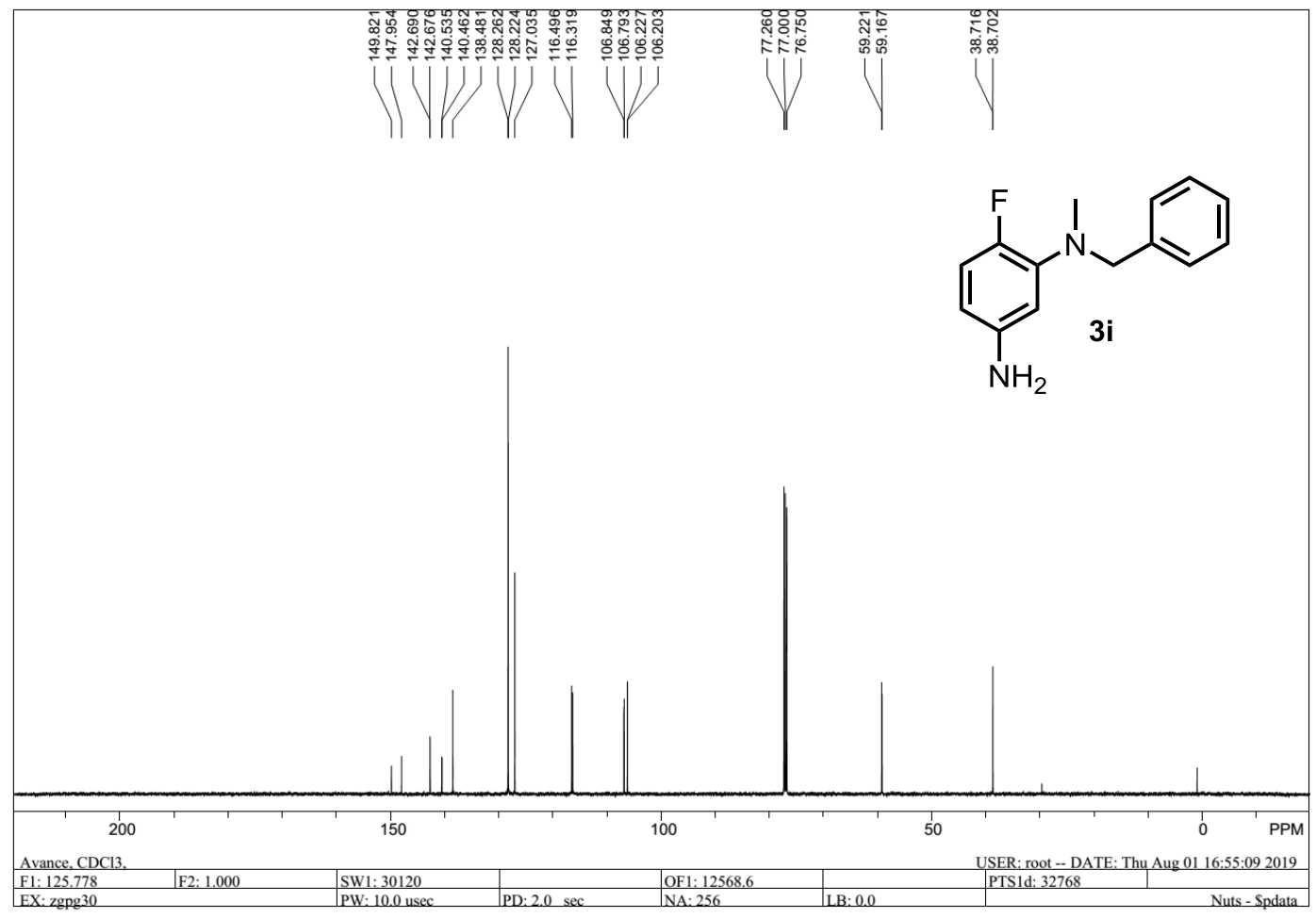


${ }^{19} \mathrm{~F}$ NMR (470 MHz, $\mathrm{CDCl}_{3}$ ):

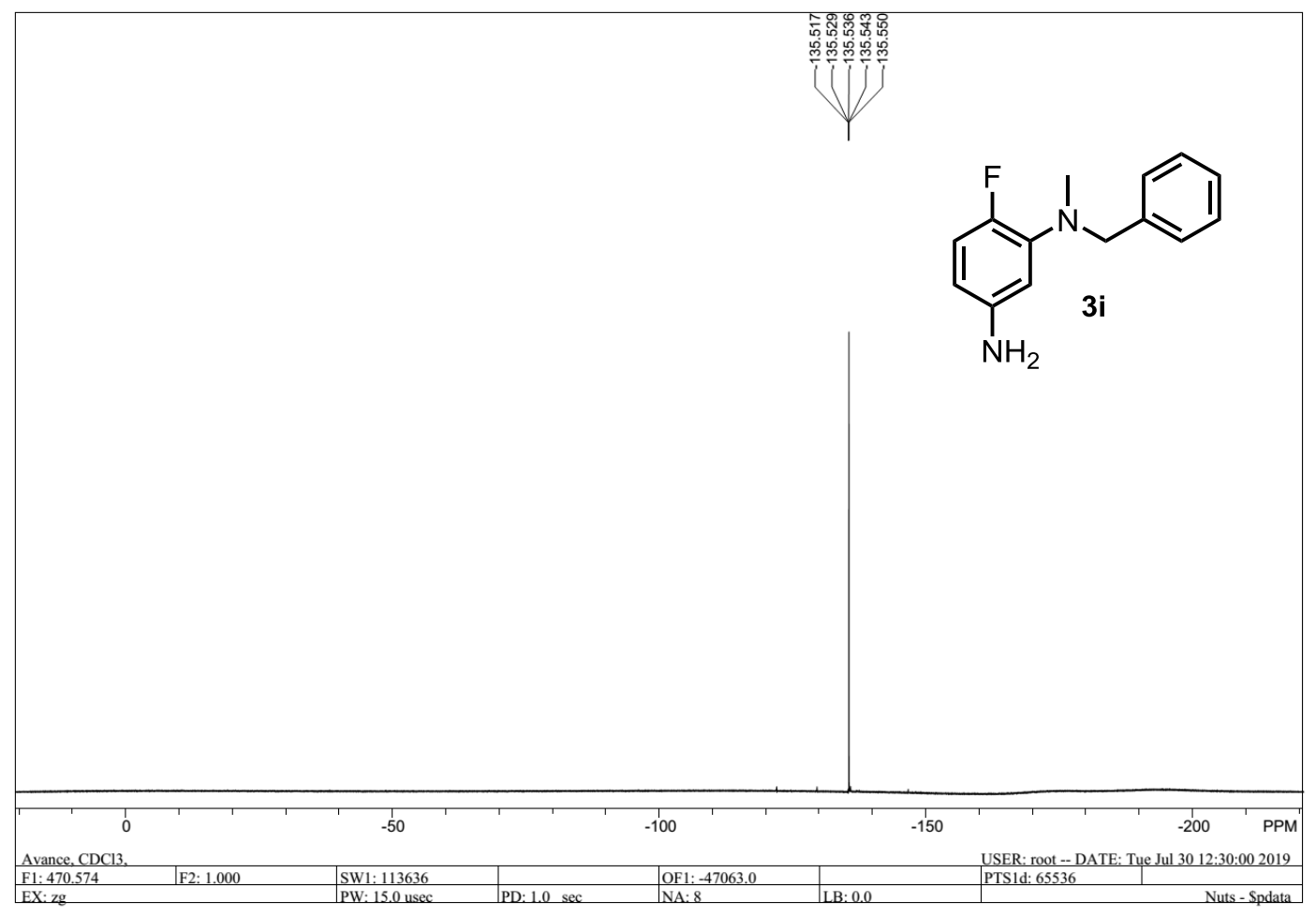


${ }^{1} \mathrm{H}$ NMR $\left(500 \mathrm{MHz}, \mathrm{CDCl}_{3}\right)$ :

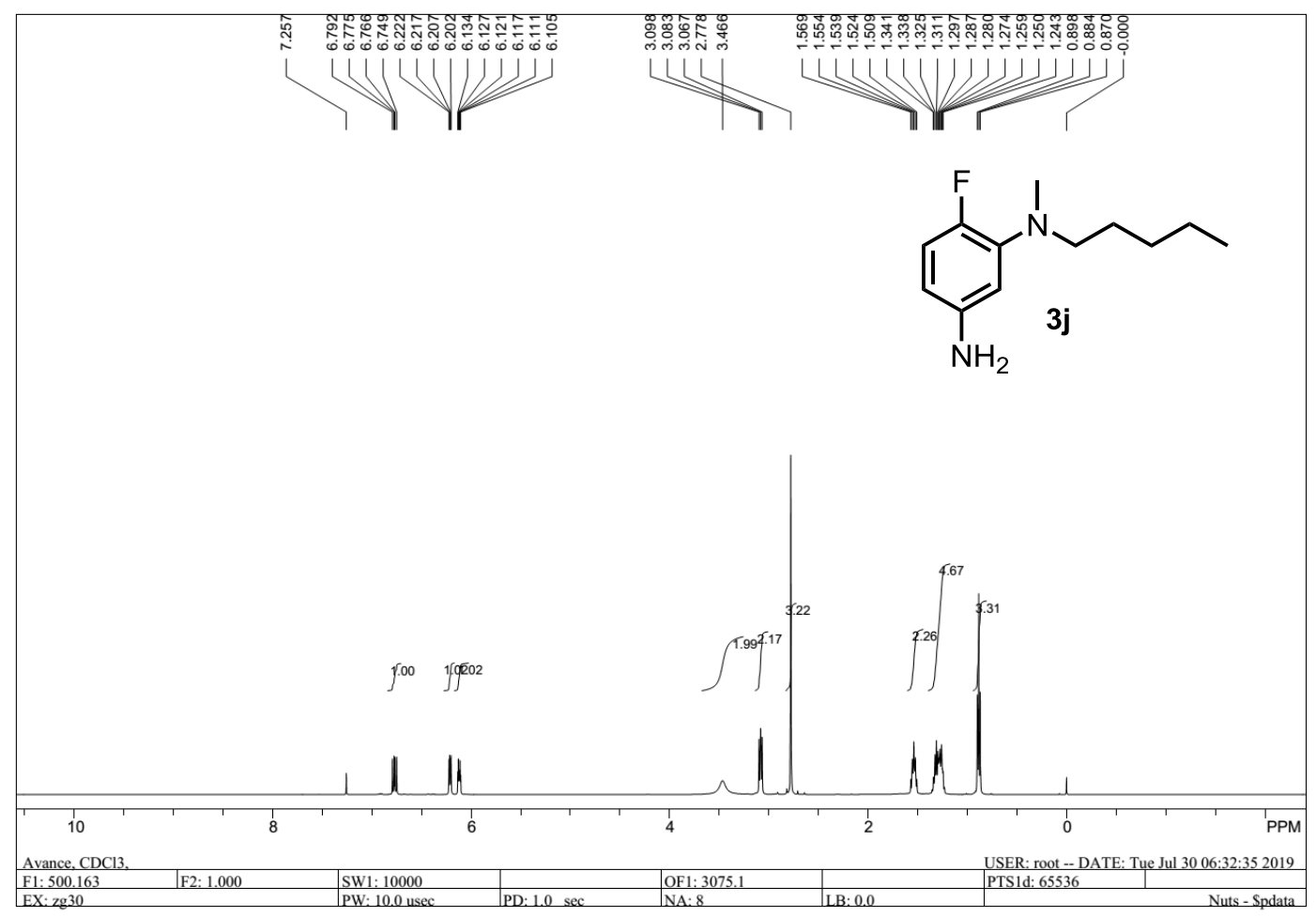

${ }^{13} \mathrm{C}$ NMR $\left(125 \mathrm{MHz}, \mathrm{CDCl}_{3}\right)$ :

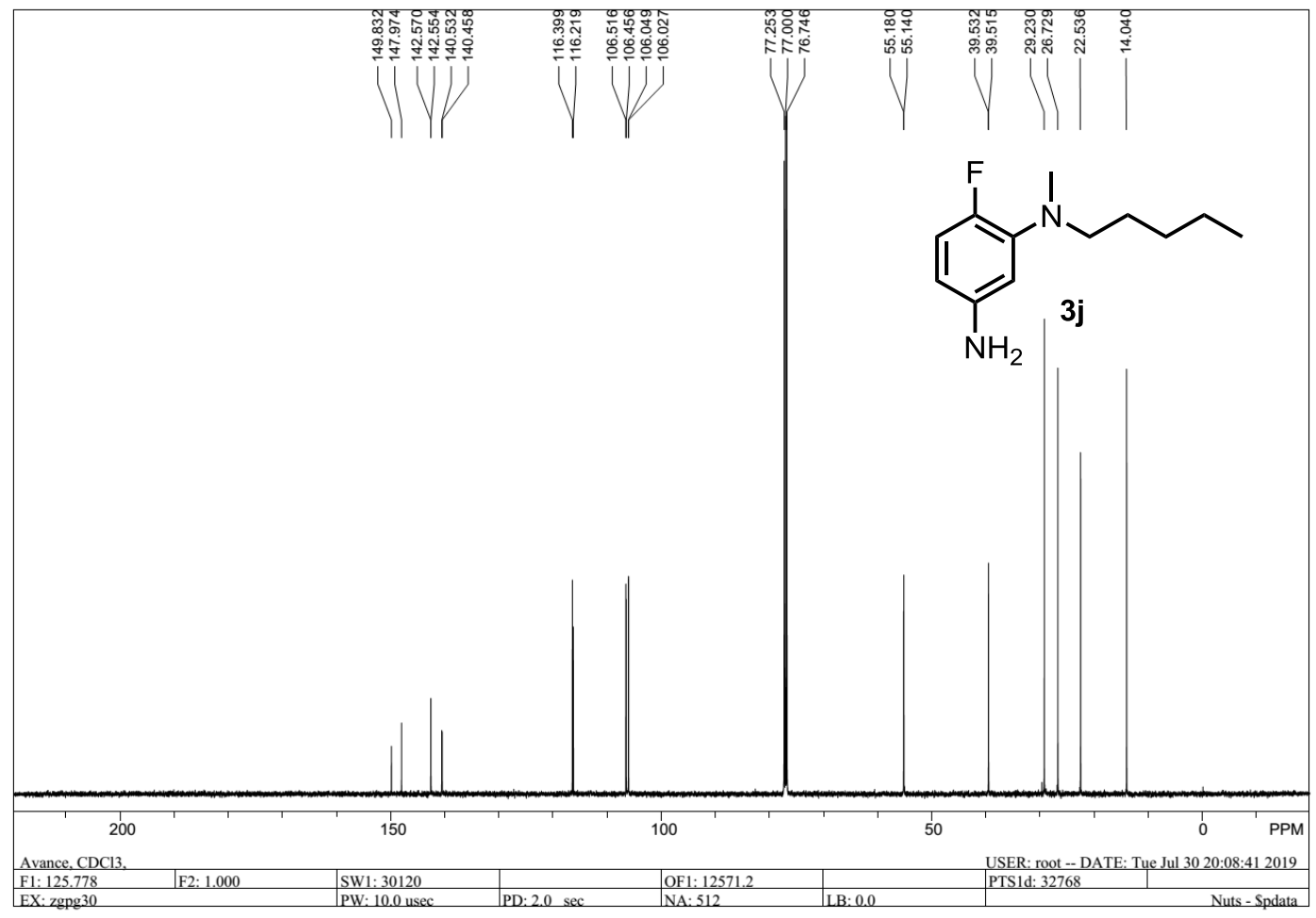


${ }^{19} \mathrm{~F}$ NMR (470 MHz, $\mathrm{CDCl}_{3}$ ):

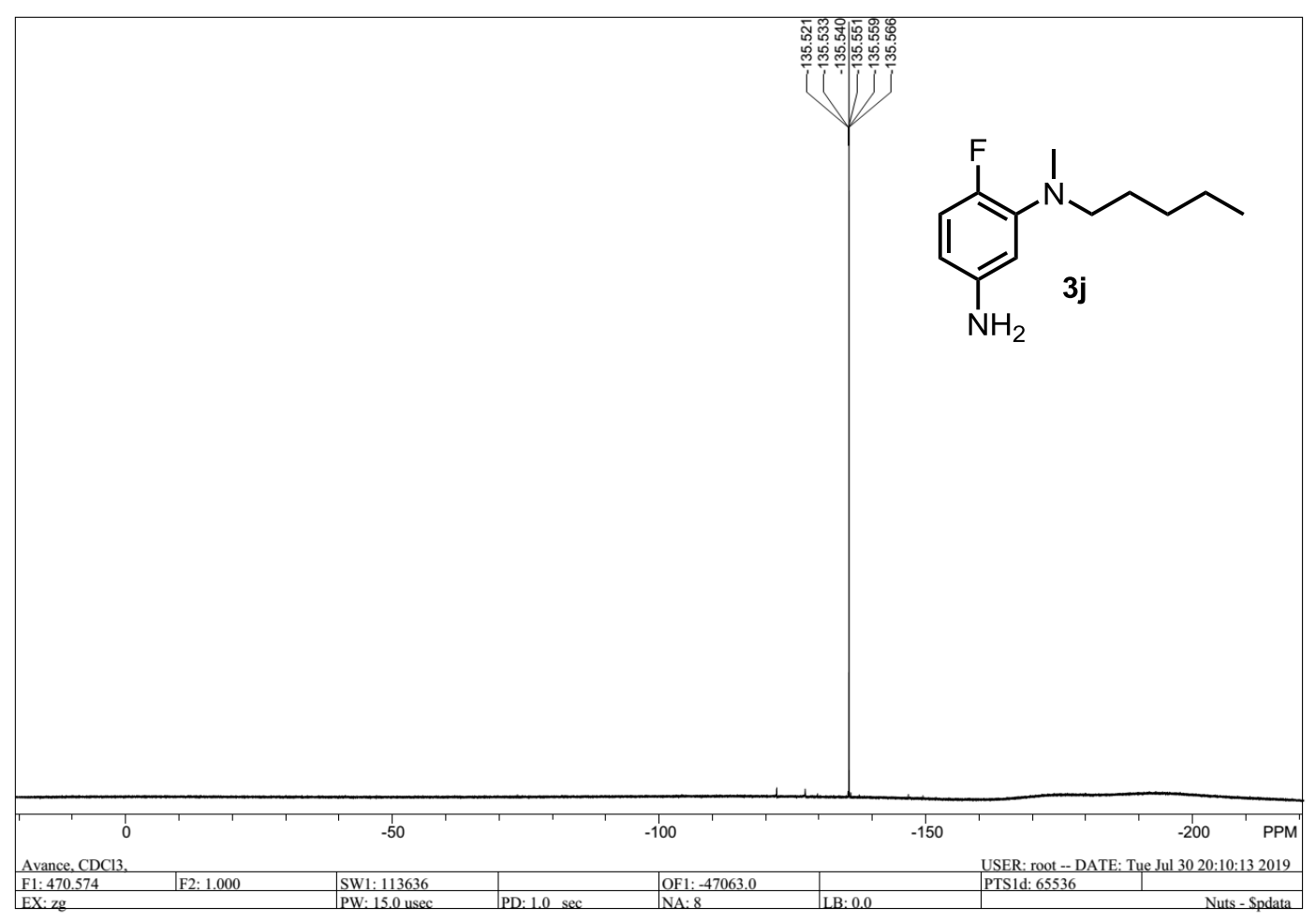


${ }^{1} \mathrm{H}$ NMR (500MHz, $\left.\mathrm{CDCl}_{3}\right)$ :

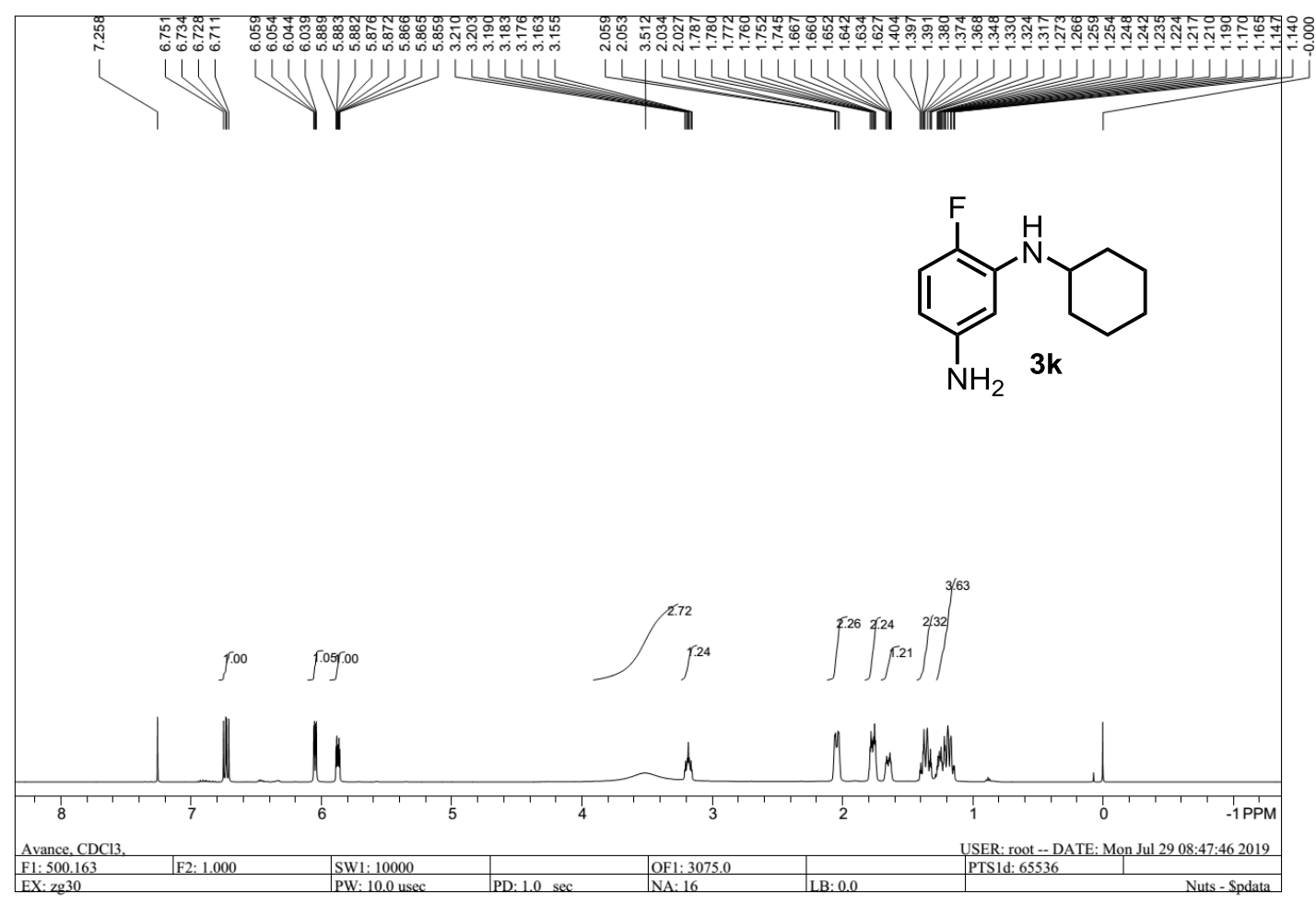

${ }^{13} \mathrm{C} \mathrm{NMR}\left(125 \mathrm{MHz}, \mathrm{CDCl}_{3}\right)$ :

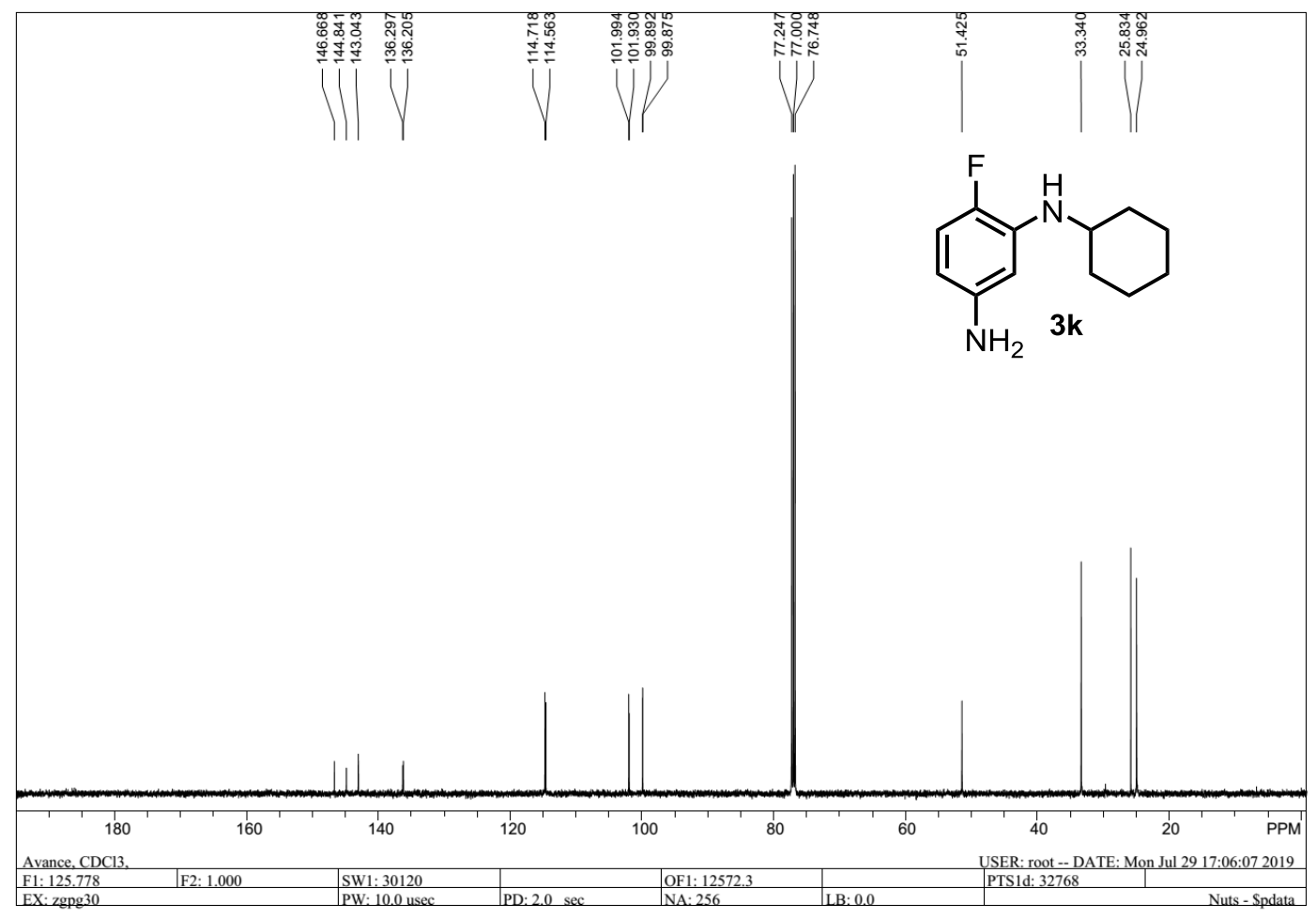


${ }^{19} \mathrm{~F}$ NMR (470 MHz, $\mathrm{CDCl}_{3}$ ):

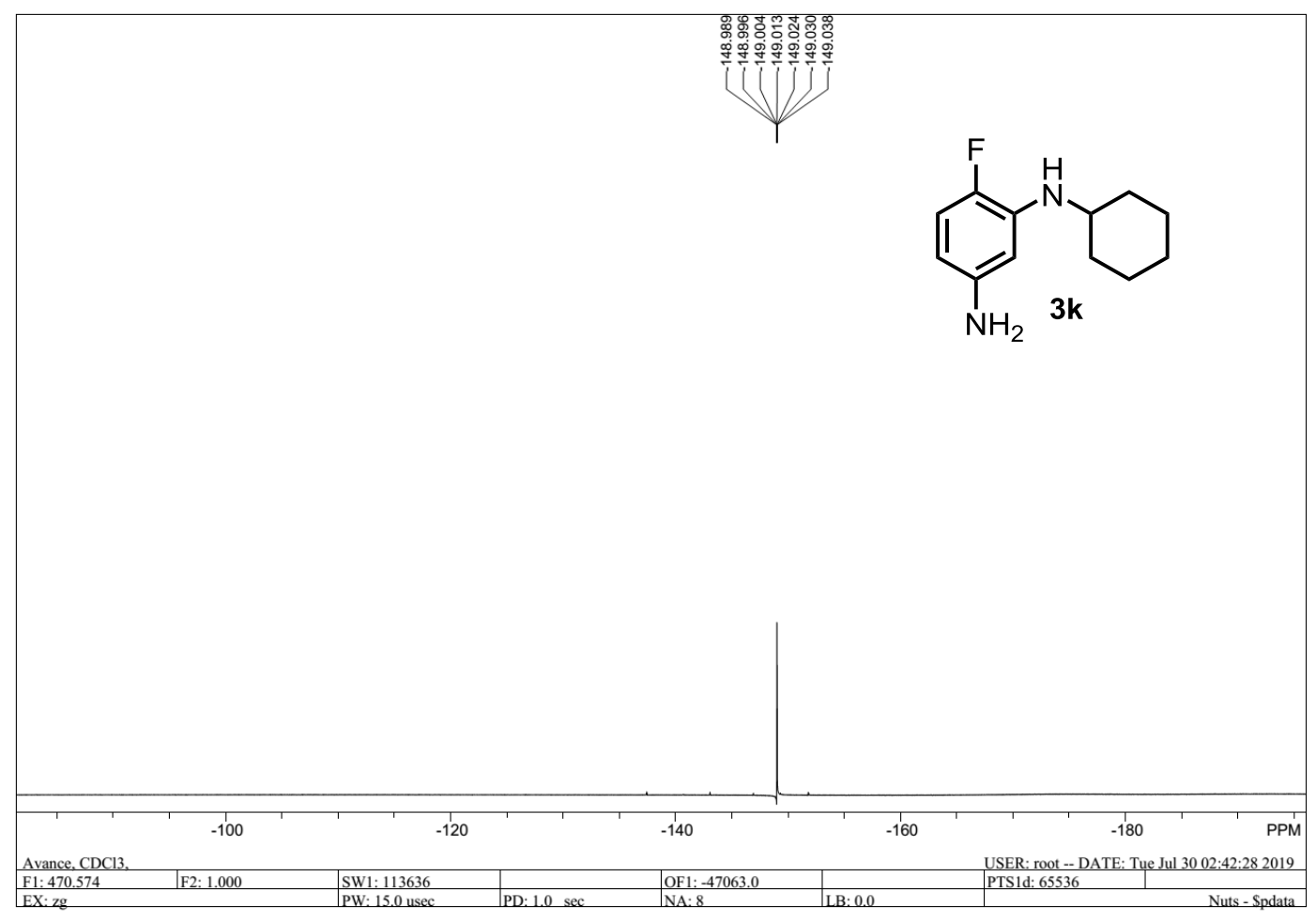


${ }^{1} \mathrm{H}$ NMR (500MHz, $\mathrm{CDCl}_{3}$ ):

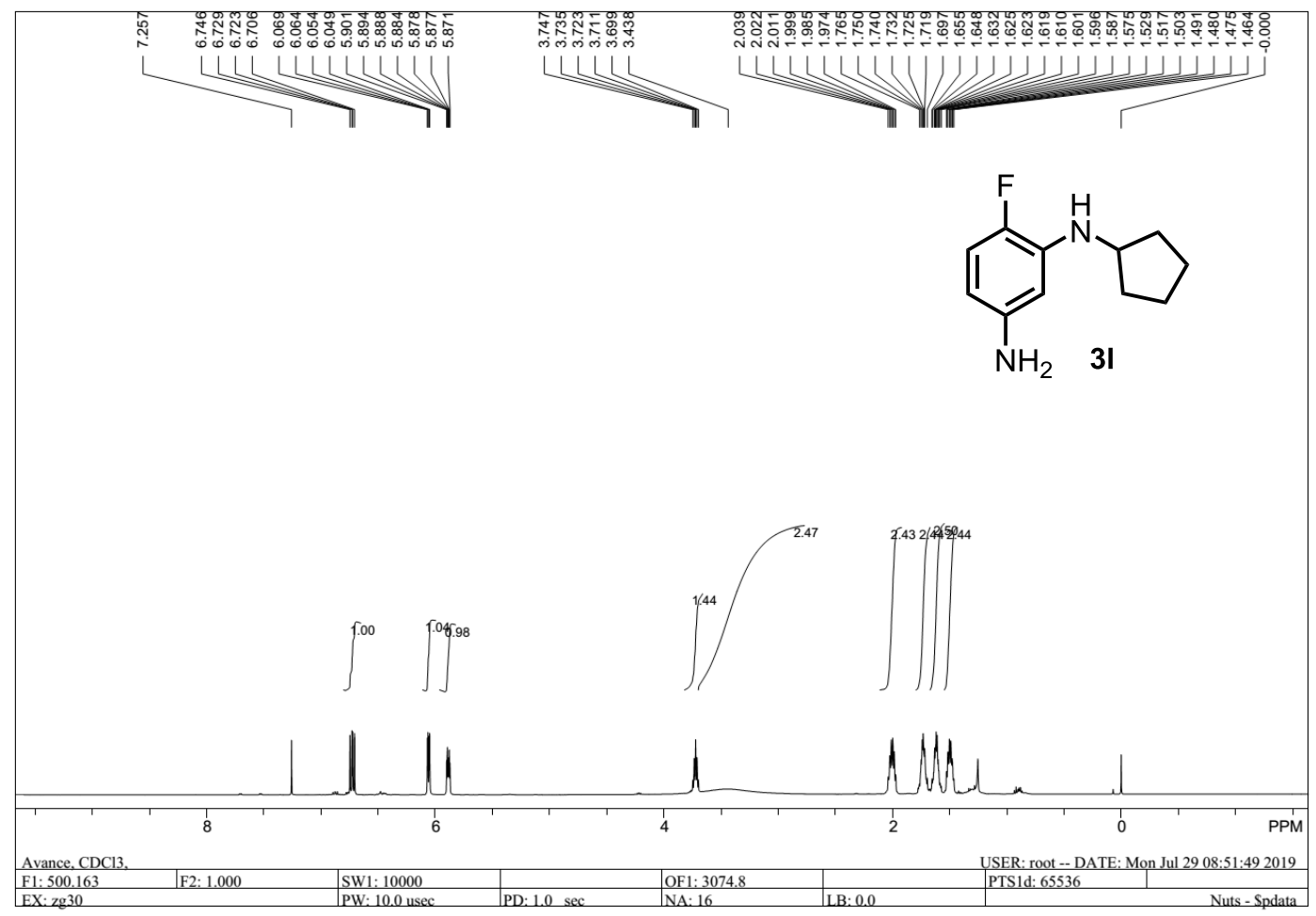

${ }^{13} \mathrm{C}$ NMR $\left(125 \mathrm{MHz}, \mathrm{CDCl}_{3}\right)$ :

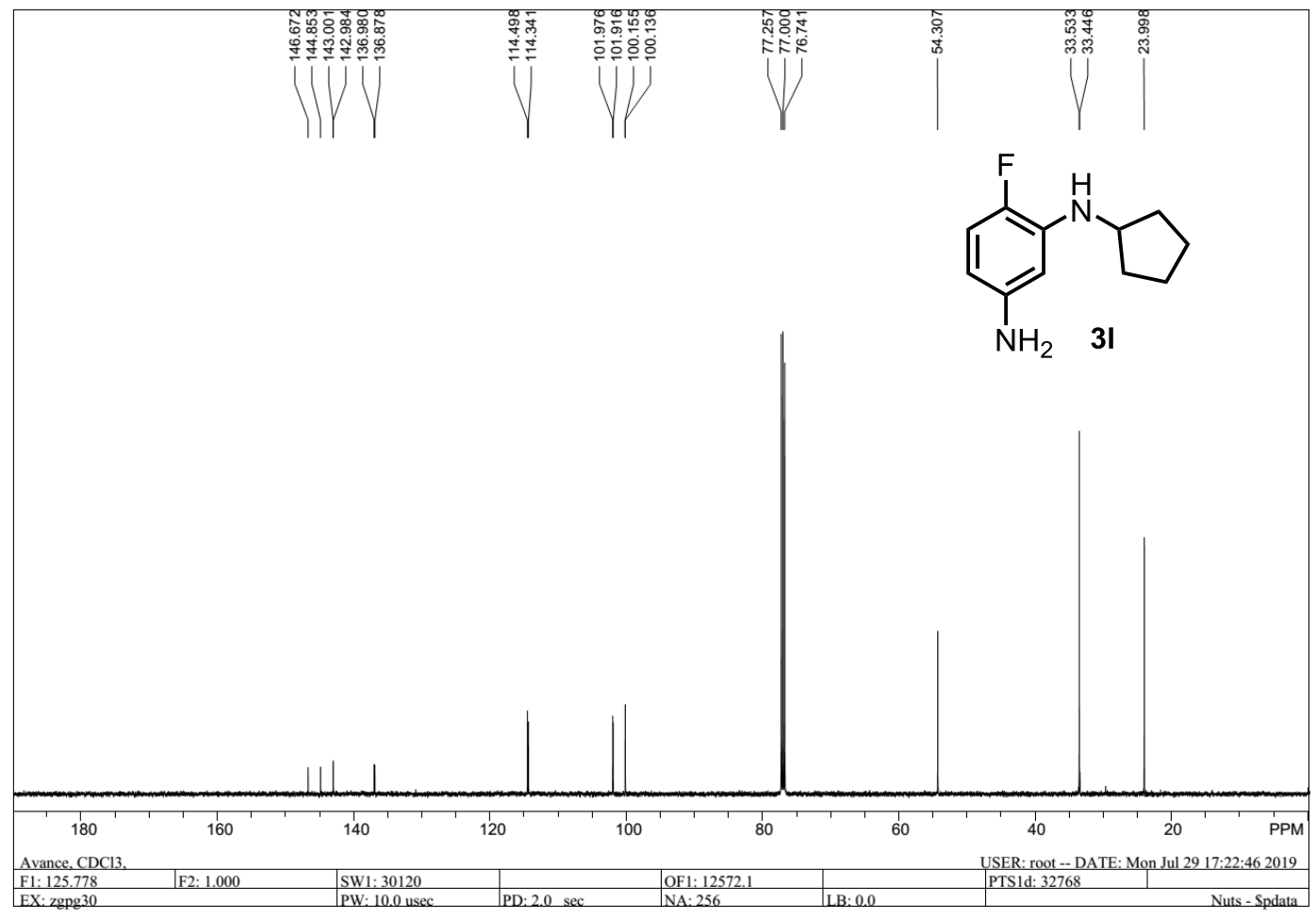


${ }^{19} \mathrm{~F}$ NMR (470 MHz, $\mathrm{CDCl}_{3}$ ):

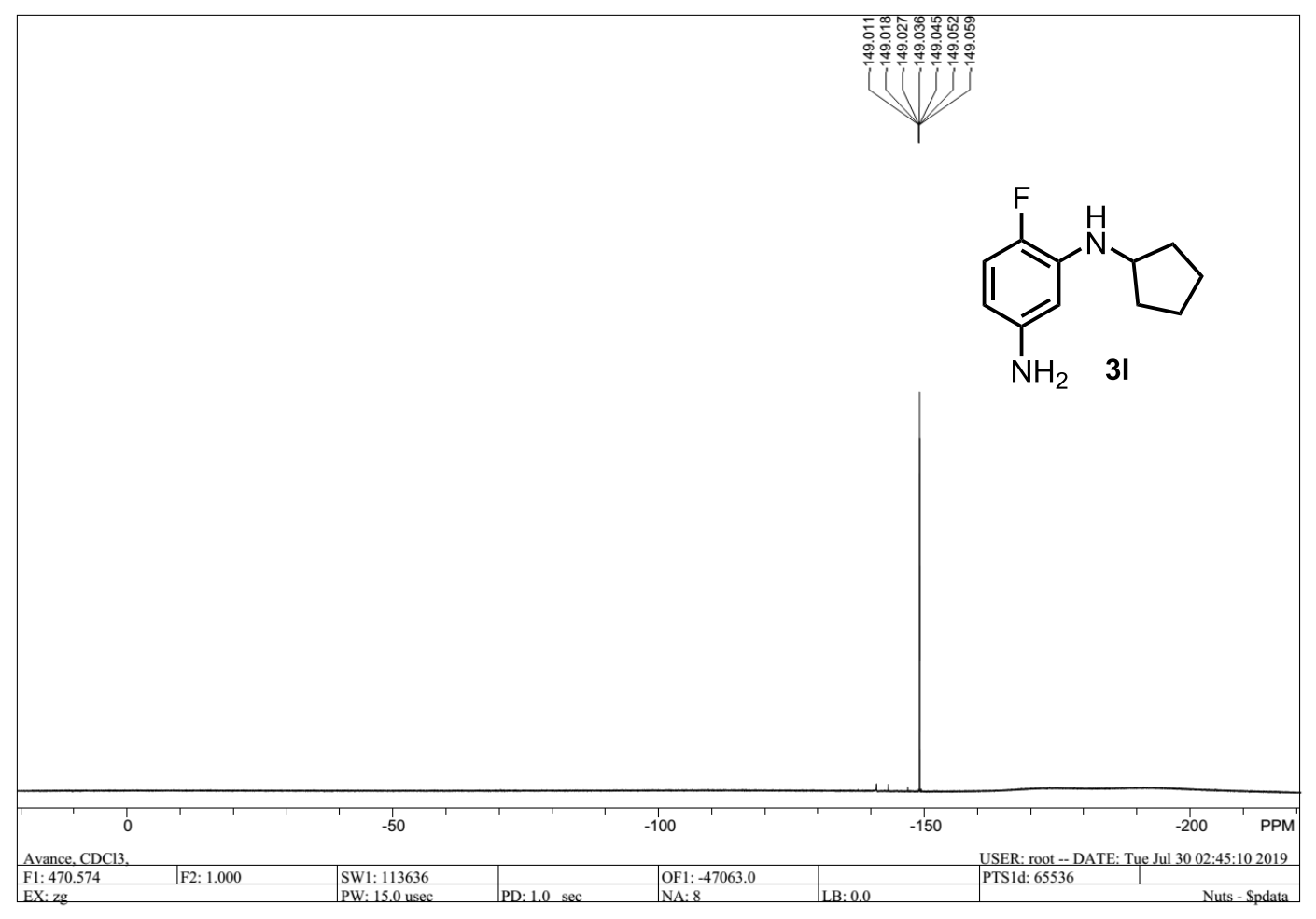


${ }^{1} \mathrm{H}$ NMR (500MHz, $\left.\mathrm{CDCl}_{3}\right)$ :

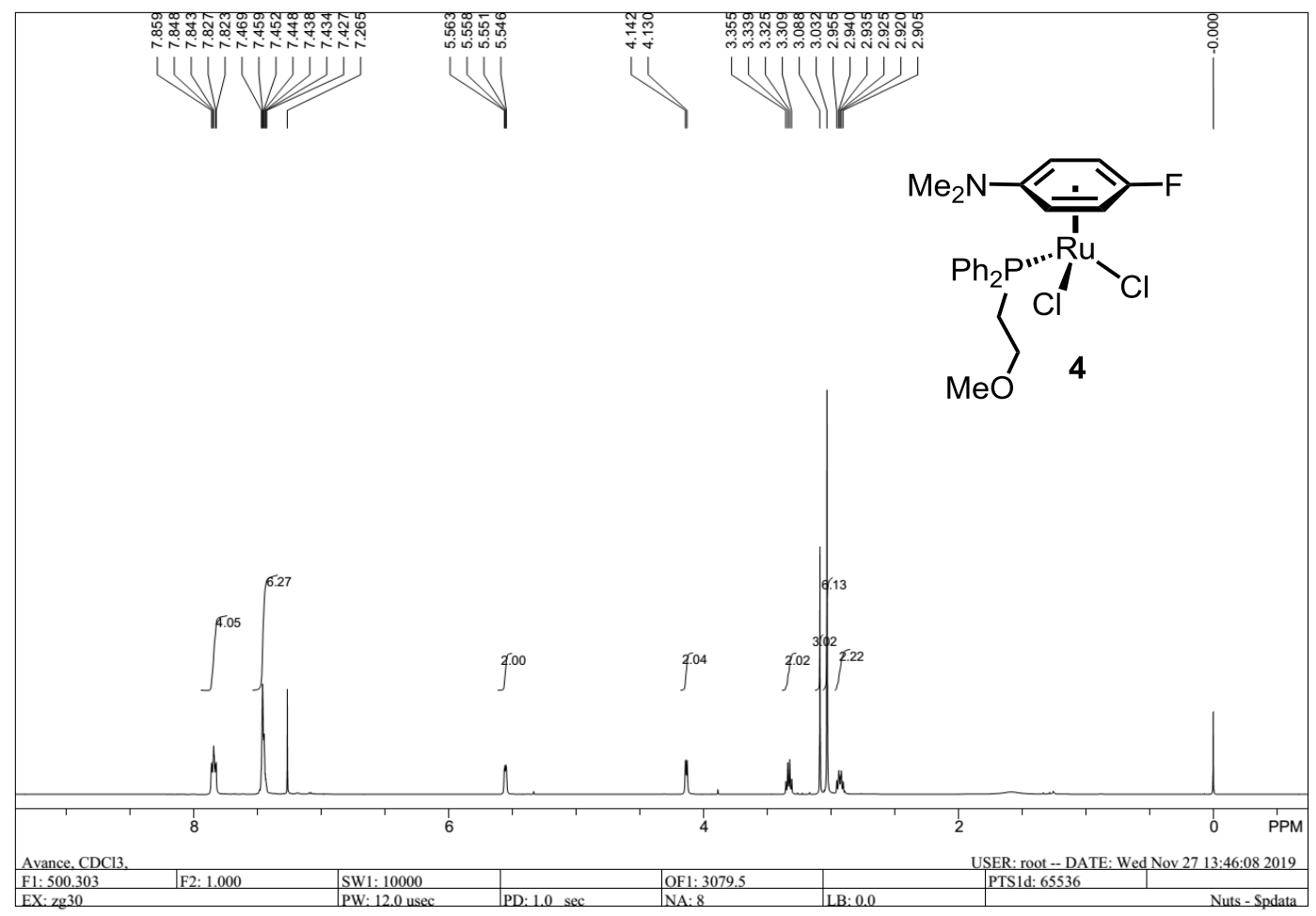

${ }^{13} \mathrm{C}$ NMR (125MHz, $\left.\mathrm{CDCl}_{3}\right)$ :

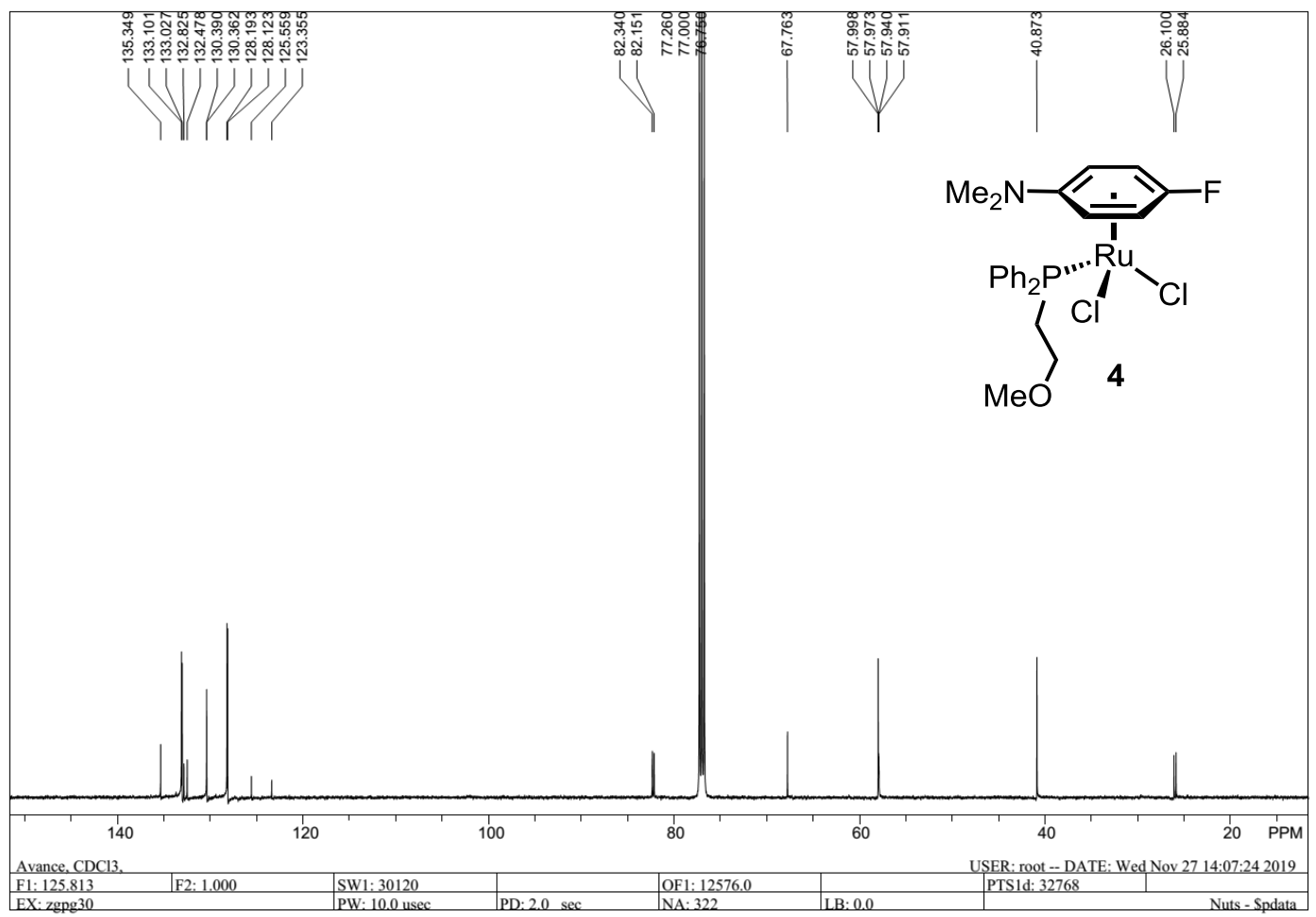


${ }^{19} \mathrm{~F}$ NMR (470 MHz, $\mathrm{CDCl}_{3}$ ):

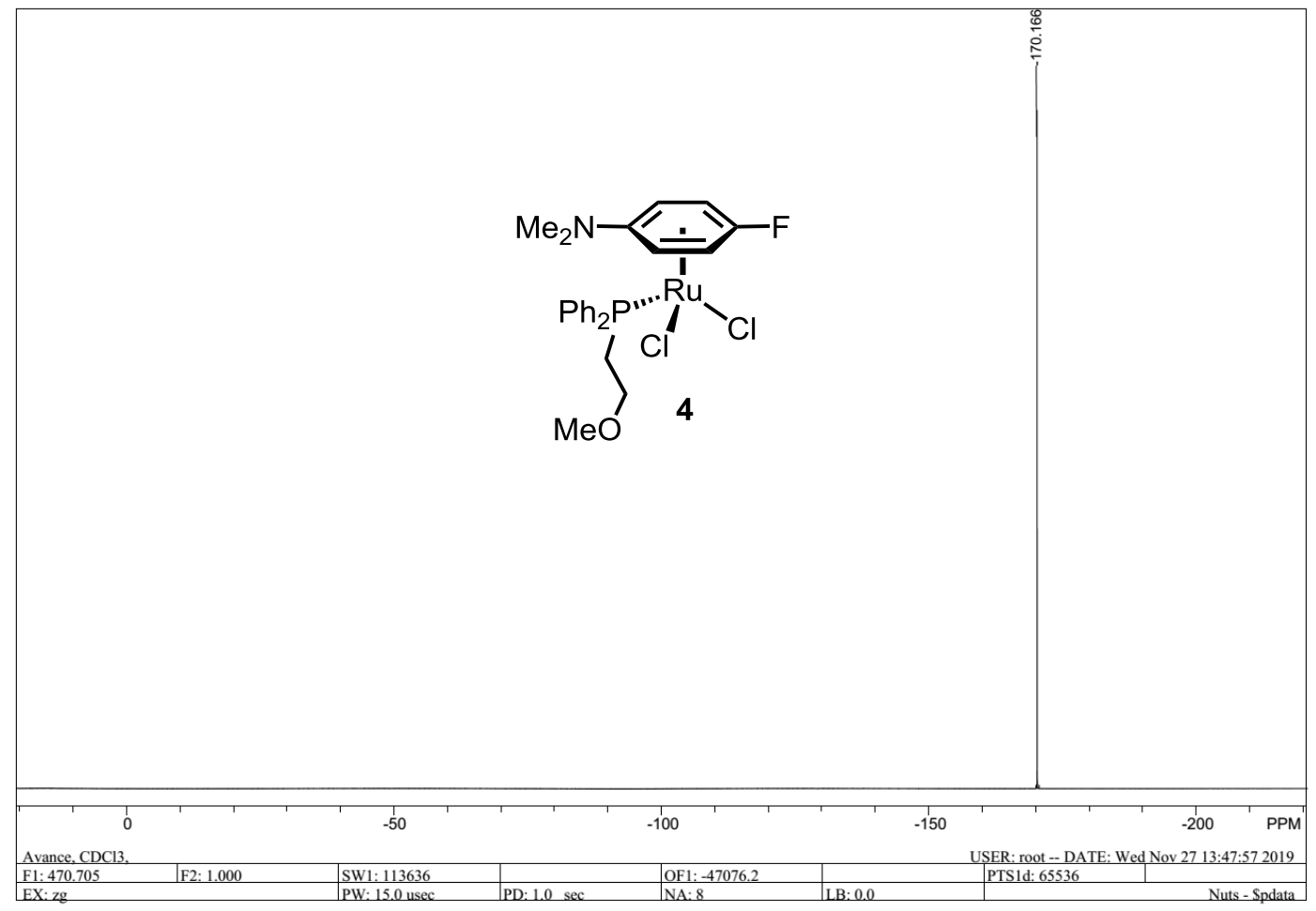

${ }^{31} \mathrm{P}$ NMR (202 MHz, $\left.\mathrm{CDCl}_{3}\right)$ :

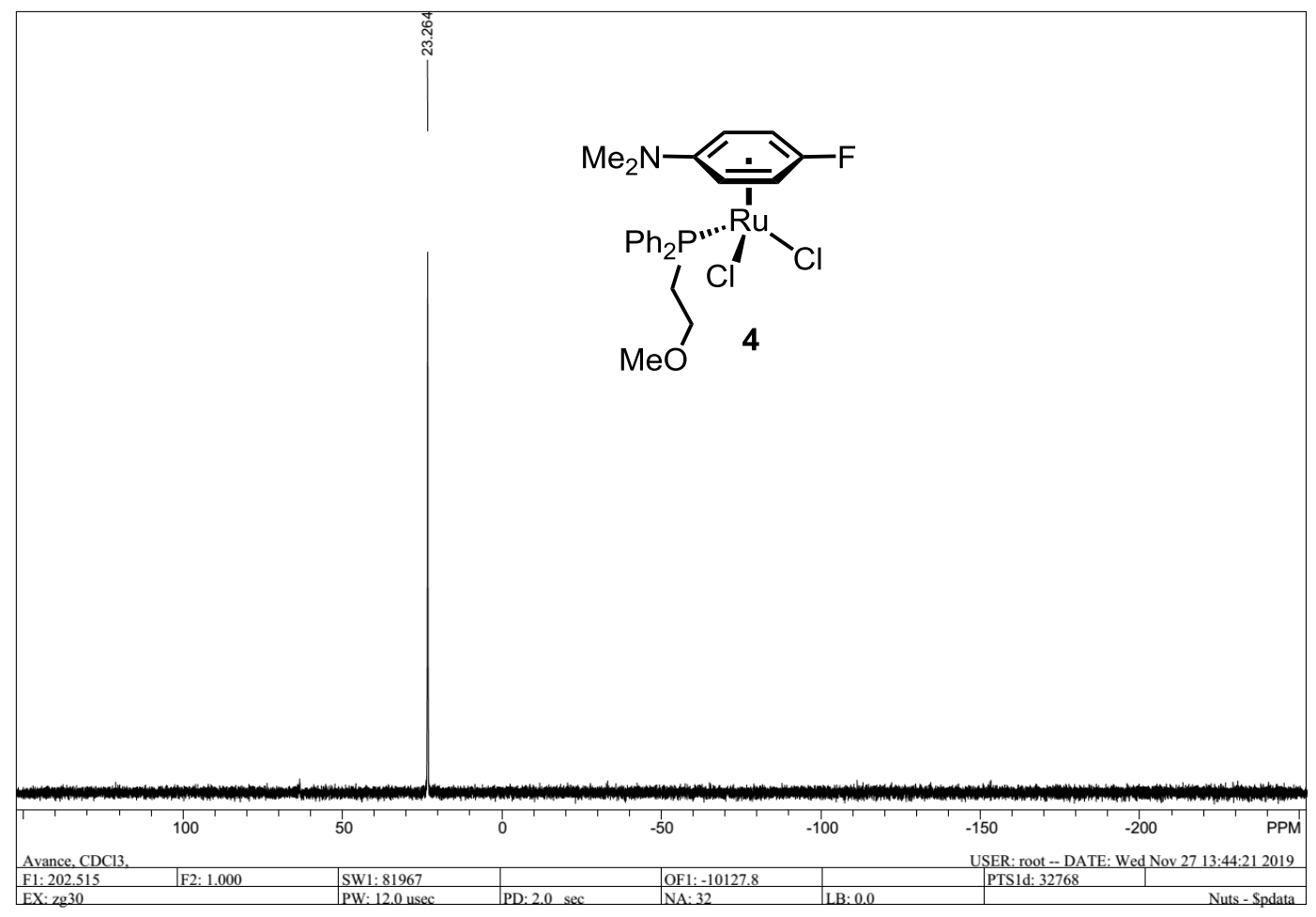


${ }^{1} \mathrm{H}$ NMR $\left(500 \mathrm{MHz}, \mathrm{CDCl}_{3}\right)$ :

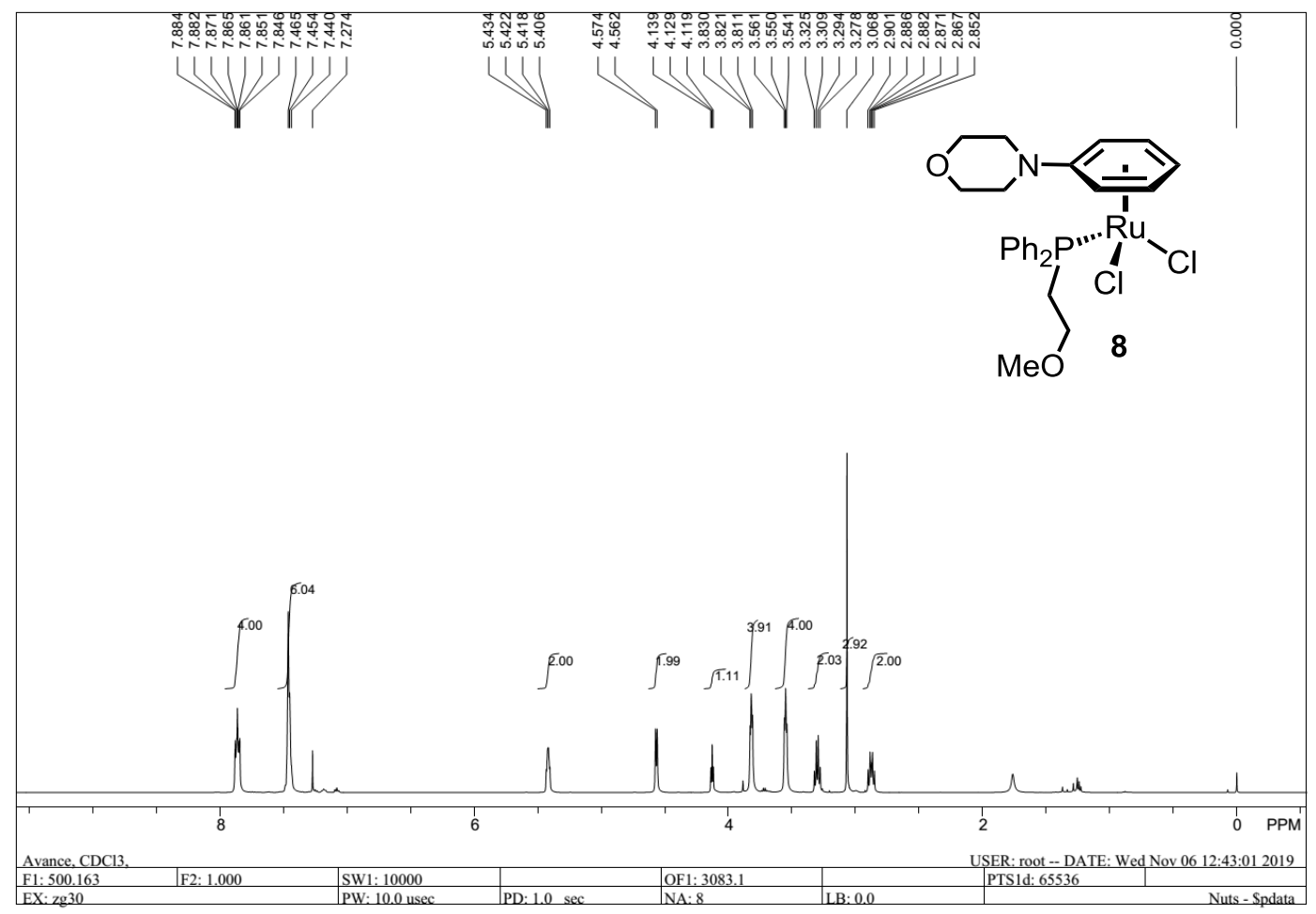

${ }^{13} \mathrm{C}$ NMR $\left(125 \mathrm{MHz}, \mathrm{CDCl}_{3}\right)$ :

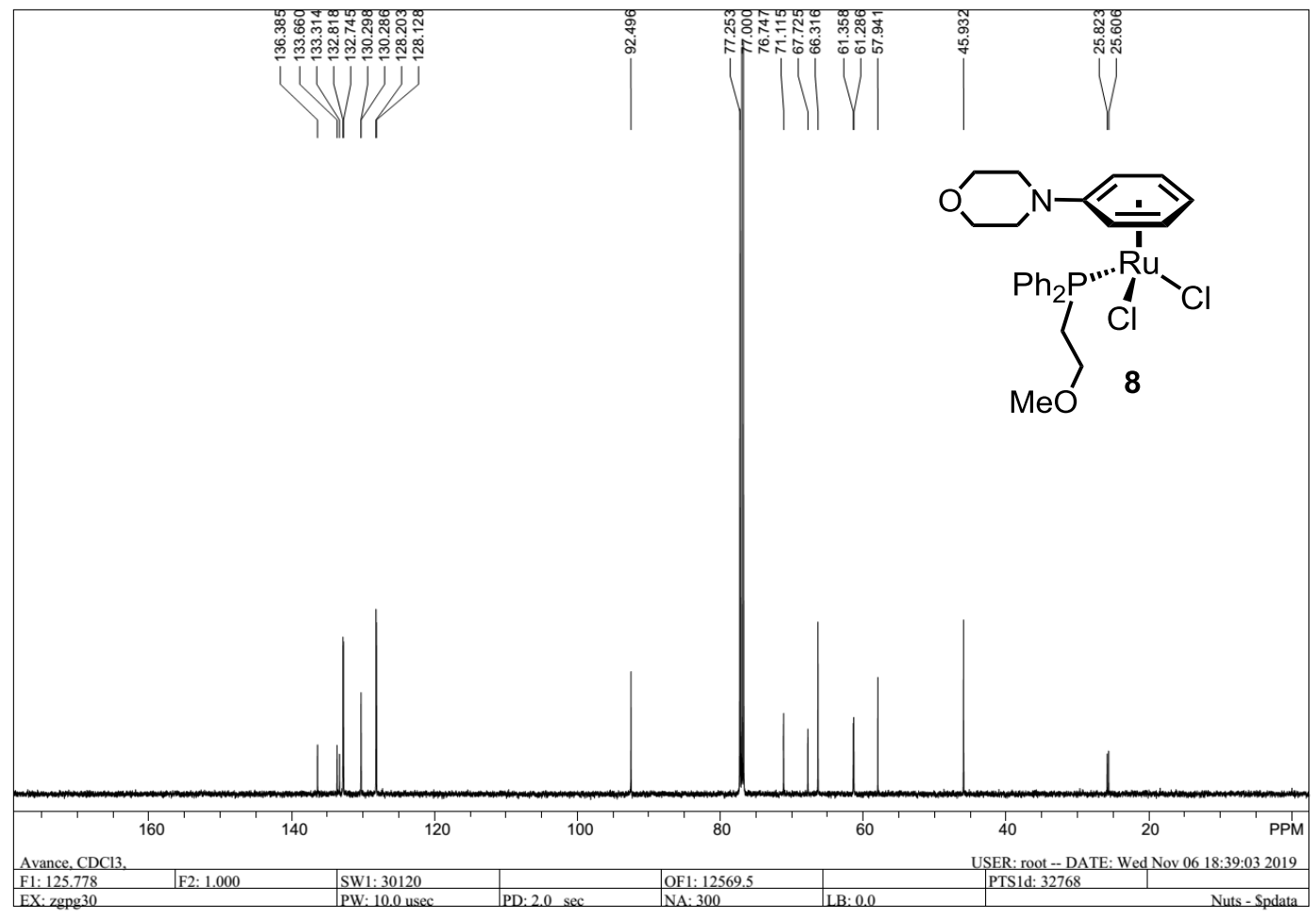


${ }^{31} \mathrm{P}$ NMR (202 MHz, $\left.\mathrm{CDCl}_{3}\right)$ :

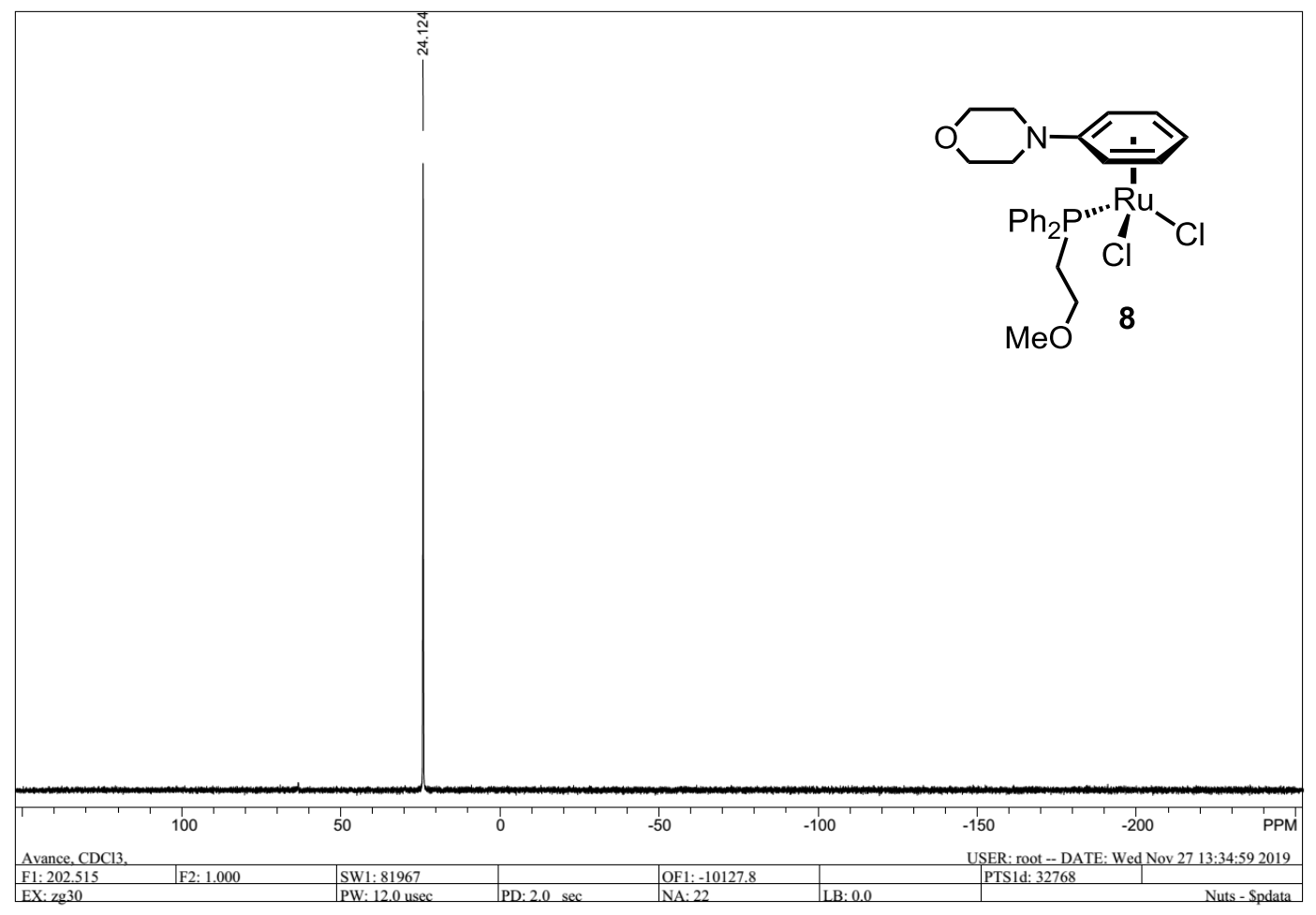


${ }^{1} \mathrm{H}$ NMR (600MHz, $\left.\mathrm{CDCl}_{3}\right)$ :

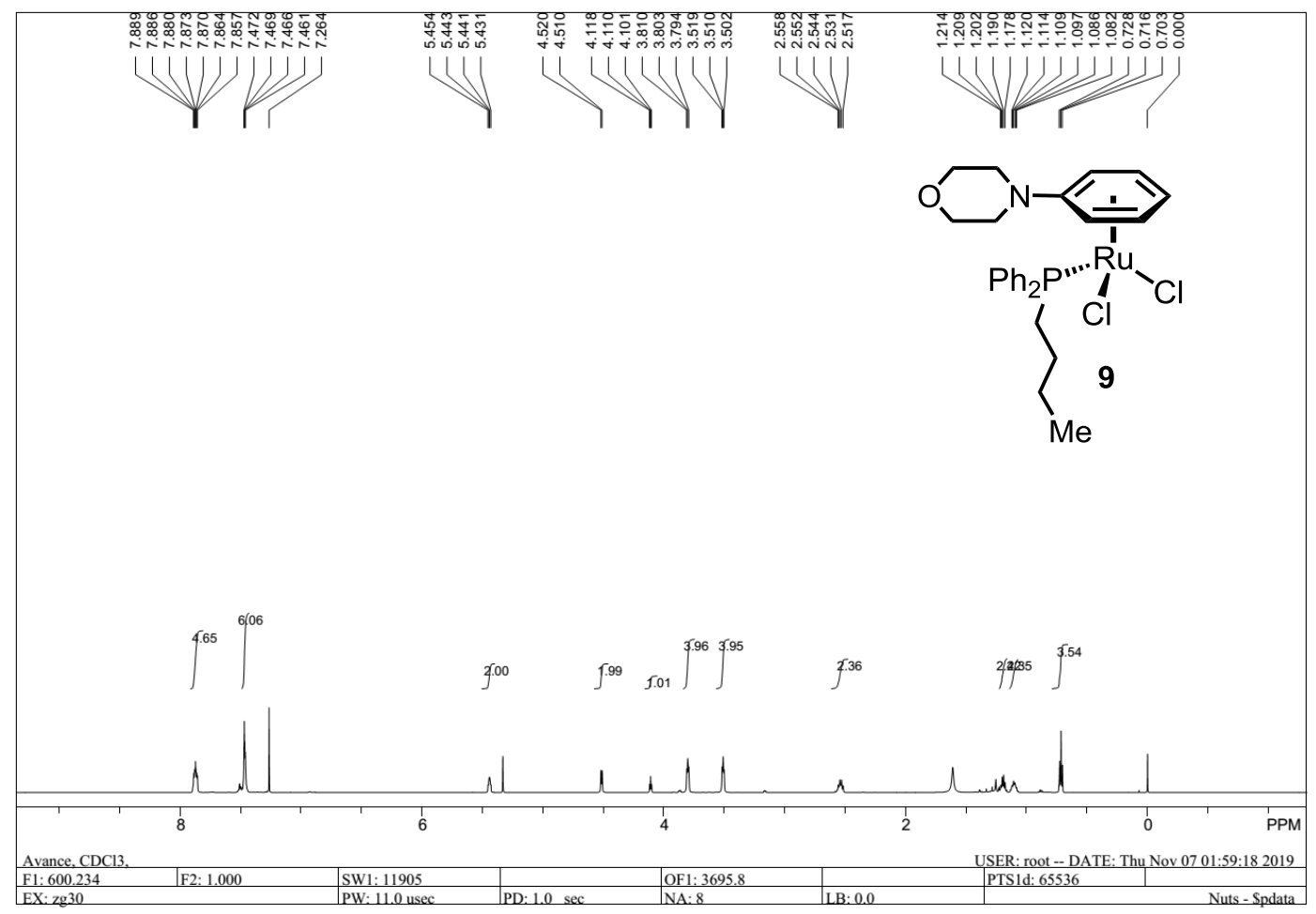

${ }^{13} \mathrm{C}$ NMR (150MHz, $\left.\mathrm{CDCl}_{3}\right)$ :

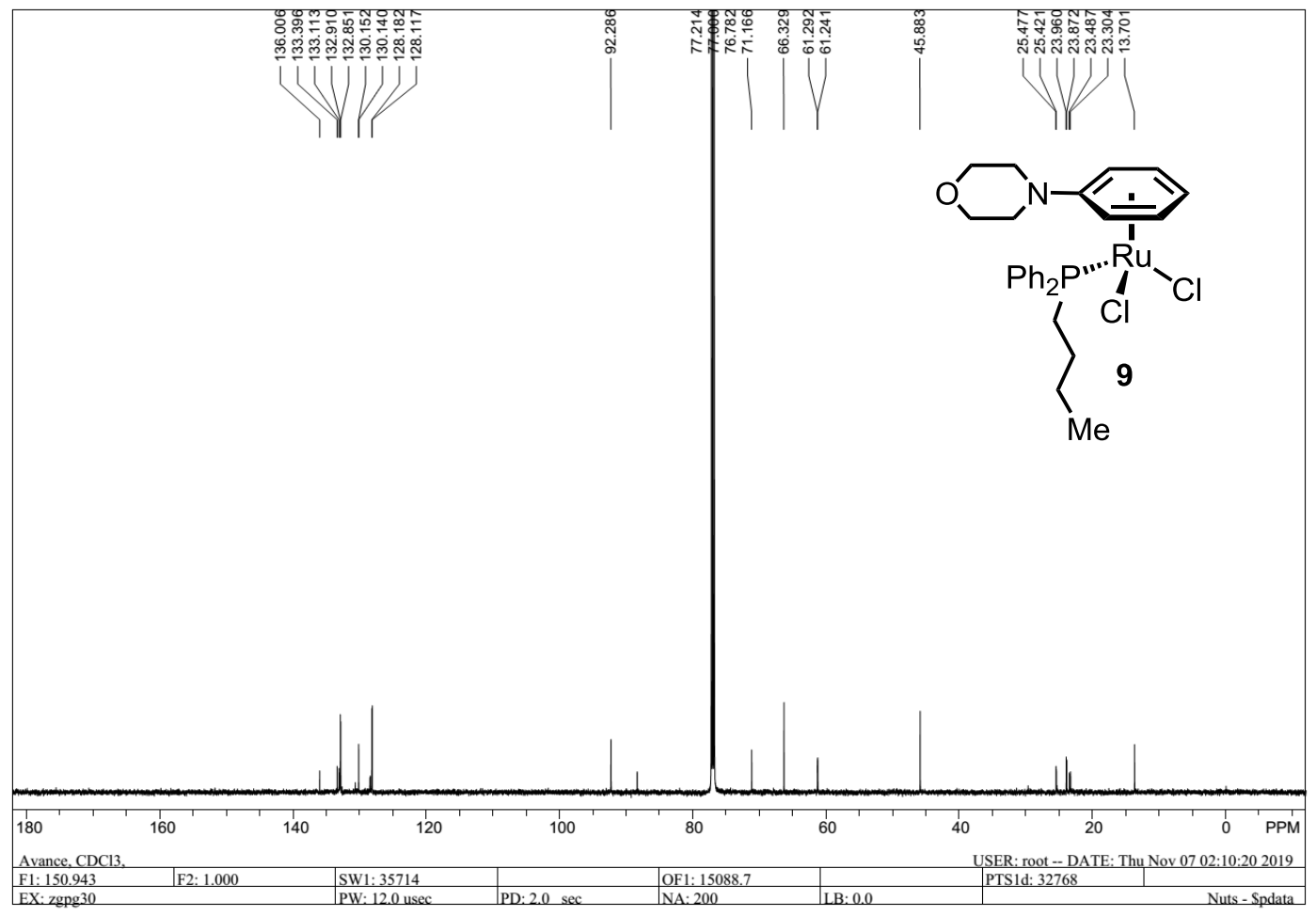


${ }^{31}$ P NMR (202 MHz, $\left.\mathrm{CDCl}_{3}\right)$ :

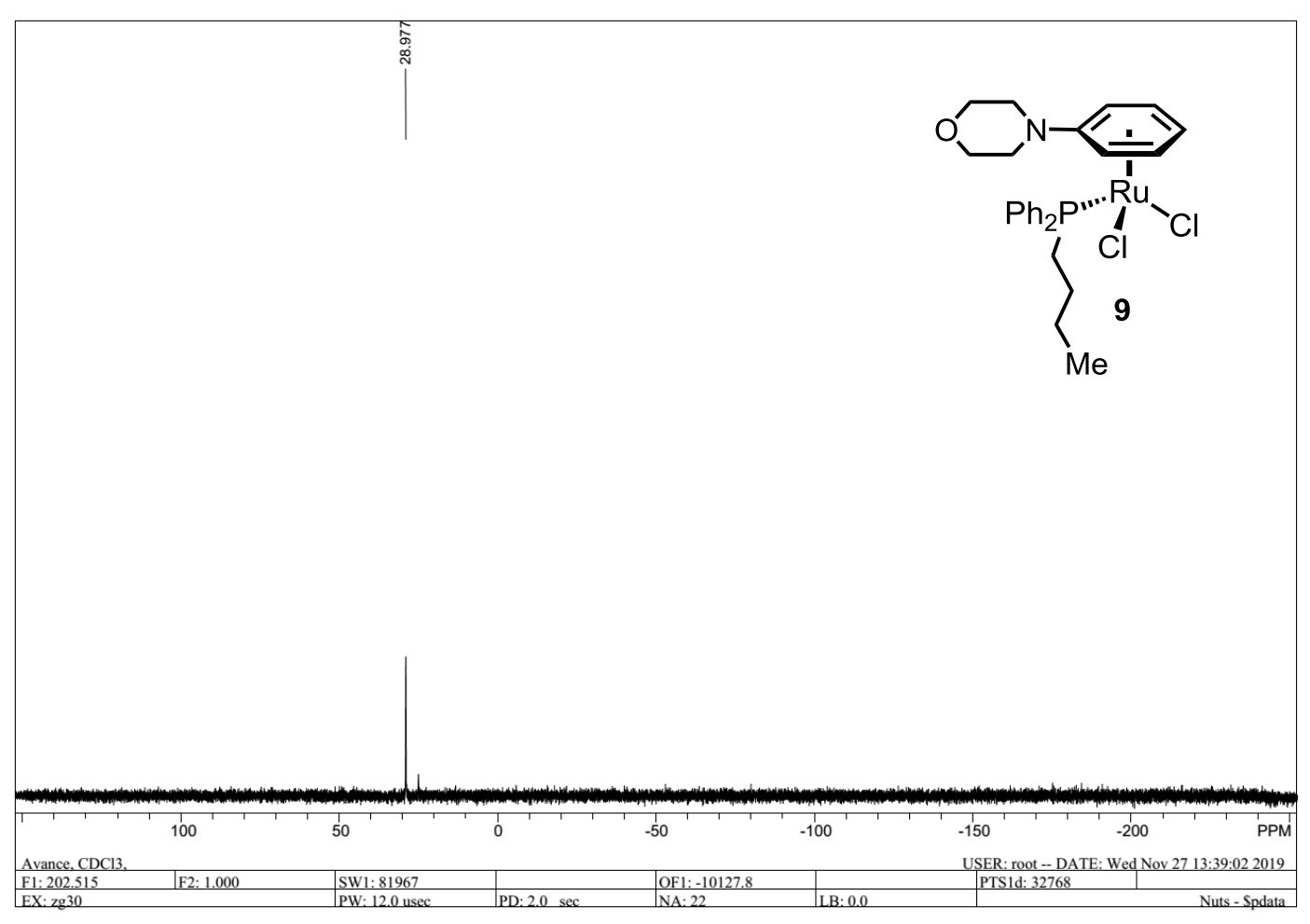




\section{References}

1. L5: (a) Huang, H.; Kang, J. Y. J. Org. Chem. 2017, 82, 6604-6614. L6, L11 and L17: (b) Lindner, E.; Meyer, S.; Wegner, P.; Karle, B.; Sickinger, A.; Steger, B. J. Organomet. Chem. 1987, 335, 59-70. L7: (c) Li, Y.; Lu, L.; Das, S.; Pisiewicz, S.; Junge, K.; Beller, M. J. Am. Chem. Soc. 2012, 134, 18325-18329. L8: (d) McEwen, W. E.; Janes, A. B.; Knapczyk, J. W.; Kyllingstad, V. L.; Shiau, W.; Shore, S.; Smith, J. H. J. Am. Chem. Soc. 1978, 100, 7304-7311. L9: (e) Gelman, D.; Jiang, L.; Buchwald, S. L. Org. Lett. 2003, 5, 2315-2318. L10: (f) Terfort, A.; Brunner, H. J. Chem. Soc., Perkin Trans. 1, 1996, 1467-1479. L12: (g) Muller, G.; Sainz, D. J. Organomet. Chem. 1995, 495, 103-111. L13: (h) Wiester, M. J.; Braunschweig, A. B.; Yoo, H.; Mirkin, C. A. Inorg. Chem. 2010, 49, 7188-7196. L14: (i) Johansson, C.; Lloyd-Jones, G. C.; Norrby, P. Tetrahedron: Asymmetry 2010, 21, 1585-1592. L16: (j) Yuan, J.; Hu, H.; Cui, C. Chem. Eur. J. 2016, 22, 5778-5785. L18: (k) Bolzati, C.; Boschi, A.; Uccelli, L.; Tisato, F.; Refosco, F.; Cagnolini, A.; Duatti, A.; Prakash, S.; Bandoli, G.; Vittadini, A. J. Am. Chem. Soc. 2002, 124, 11468-11479.

2. (a) Xiao, Q.; Tian, L.; Tan, R.; Xia, Y.; Qiu, D.; Zhang, Y.; Wang, J. Org. Lett. 2012, 14, 4230-4233. (b) Begam, H. M.; Choudhury, R.; Behera, A.; Jana, R. Org. Lett. 2019, 21, 4651-4656. (c) Kim, M.; Shin, T.; Lee, A.; Kim, H. Organometallics 2018, 37, 3253-3258. (d) Li, W.; Liu, W.; Leonard, D. K.; Rabeah, J.; Junge, K.; Brückner, A.; Beller, M. Angew. Chem. Int. Ed. 2019, 58, 10693-10697. (e) Li, H. L.; Kuninobu, Y.; Kanai, M. Angew. Chem. Int. Ed. 2017, 56, 1495-1499. (f) Shi, L.; Wang, M.; Fan, C.-A.; Zhang, F.-M.; Tu, Y.-Q. Org. Lett. 2003, 5, 3515-3517. (g) Gao, J.; Bhunia, S.; Wang, K.; Gan, L.; Xia, S.; Ma, D. Org. Lett. 2017, 19, 2809-2812. (h) Nishizawa, A.; Takahira, T.; Yasui, K.; Fujimoto, H.; Iwai, T.; Sawamura, M.; Chatani, N.; Tobisu, M. J. Am. Chem. Soc. 2019, 141, 7261-7265. (i) Huang, X.; Anderson, K. W.; Zim, D.; Jiang, L.; Klapars, A.; Buchwald, S. L.; J. Am. Chem. Soc. 2003, 125, 6653-6655. 